\title{
Operationalizing Clean Development Mechanism baselines: A case study of China's electrical sector
}

\author{
by \\ Paul A. Steenhof \\ A thesis submitted to \\ the Faculty of Graduate Studies and Research \\ in partial fulfillment of the requirements for the degree of \\ Doctor of Philosophy \\ Department of Geography and Environmental Studies \\ Carleton University \\ Ottawa, Ontario \\ September 2006
}

(C) copyright 2006

Paul A. Steenhof 


$\begin{array}{ll}\begin{array}{l}\text { Library and } \\ \text { Archives Canada }\end{array} & \begin{array}{l}\text { Bibliothèque et } \\ \text { Archives Canada }\end{array} \\ \begin{array}{l}\text { Published Heritage } \\ \text { Branch }\end{array} & \begin{array}{l}\text { Direction du } \\ \text { Patrimoine de l'édition }\end{array} \\ \begin{array}{l}\text { 395 Wellington Street } \\ \text { Ottawa ON K1A 0N4 }\end{array} & \begin{array}{l}\text { 395, rue Wellington } \\ \text { Ottana ON K1A ON4 } \\ \text { Canada Oa }\end{array}\end{array}$

Your file Votre référence ISBN: 978-0-494-18237-6 Ourfile Notre référence ISBN: 978-0-494-18237-6

NOTICE:

The author has granted a nonexclusive license allowing Library and Archives Canada to reproduce, publish, archive, preserve, conserve, communicate to the public by telecommunication or on the Internet, loan, distribute and sell theses worldwide, for commercial or noncommercial purposes, in microform, paper, electronic and/or any other formats.

The author retains copyright ownership and moral rights in this thesis. Neither the thesis nor substantial extracts from it may be printed or otherwise reproduced without the author's permission.
AVIS:

L'auteur a accordé une licence non exclusive permettant à la Bibliothèque et Archives Canada de reproduire, publier, archiver, sauvegarder, conserver, transmettre au public par télécommunication ou par l'Internet, prêter, distribuer et vendre des thèses partout dans le monde, à des fins commerciales ou autres, sur support microforme, papier, électronique et/ou autres formats.

L'auteur conserve la propriété du droit d'auteur et des droits moraux qui protège cette thèse. $\mathrm{Ni}$ la thèse ni des extraits substantiels de celle-ci ne doivent être imprimés ou autrement reproduits sans son autorisation.
In compliance with the Canadian

Privacy Act some supporting forms may have been removed from this thesis.

While these forms may be included in the document page count, their removal does not represent any loss of content from the thesis.
Conformément à la loi canadienne sur la protection de la vie privée, quelques formulaires secondaires ont été enlevés de cette thèse.

Bien que ces formulaires aient inclus dans la pagination, il n'y aura aucun contenu manquant. 
Abstract

The global carbon market is rapidly developing as the first commitment period of the Kyoto Protocol draws closer and Parties to the Protocol with greenhouse gas (GHG) emission reduction targets seek alternative ways to reduce their emissions. The Protocol includes the Clean Development Mechanism (CDM), a tool that encourages projectbased investments to be made in developing nations that will lead to an additional reduction in emissions. Due to China's economic size and rate of growth, technological characteristics, and its reliance on coal, it contains a large proportion of the global CDM potential. As China's economy modernizes, more technologies and processes are requiring electricity and demand for this energy source is accelerating rapidly. Relatively inefficient technology to generate electricity in China thereby results in the electrical sector having substantial GHG emission reduction opportunities as related to the CDM. In order to ensure the credibility of the CDM in leading to a reduction in GHG emissions, it is important that the baseline method used in the CDM approval process is scientifically sound and accessible for both others to use and for evaluation purposes. Three different methods for assessing CDM baselines and environmental additionality are investigated in the context of China's electrical sector: a method based on a historical perspective of the electrical sector (factor decomposition), a method structured upon a current perspective (operating and build margins), and a simulation of the future (dispatch analysis). Assessing future emission levels for China's electrical sector is a very challenging task given the complexity of the system, its dynamics, and that it is heavily influenced by internal and external forces, but of the different baseline methods 
investigated, dispatch modelling is best suited for the Chinese context as it is able to consider the important regional and temporal dimensions of its economy and its future development. For China, the most promising options for promoting sustainable development, one of the goals of the Kyoto Protocol, appear to be tied to increasing electrical end-use and generation efficiency, particularly clean coal technology for electricity generation since coal will likely continue to be a dominant primary fuel. 
Acknowledgements

I would like to acknowledge the very beneficial review and comments received from my supervisor Mike Brklacich and committee members Philippe Crabbe (Department of Economics, University of Ottawa) and Deniz Karman (Department of Civil and Environmental Engineering, Carleton University) while completing the analysis for the dissertation and as I was writing the final paper. This advice and review greatly advanced the quality of the research and of the dissertation. SNC Lavalin Environment Incorporated, the Natural Science and Engineering Research Council of Canada, the Ontario Graduate Scholarship Program, the Global Environmental Change and Human Security Project, and Carleton University all provided financial contributions that allowed the research to proceed. Carleton University and the Global Environmental Change and Human Security Project provided funds to present components of the research at international conferences in Changsha China (2003), Bangkok Thailand (2004) and Beijing China (2005). ICF International Incorporated provided use of the China Integrated Planning Model for the research, and Whitman Fulton of ICF International Incorporated helped facilitate the use of the model throughout the research. 


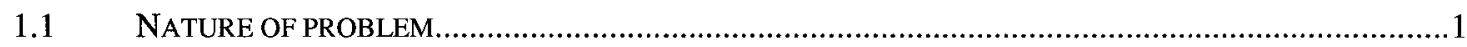

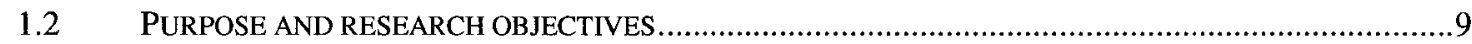

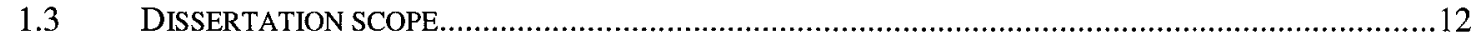

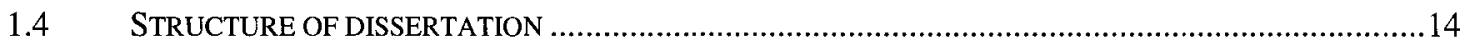

2 CHINA'S IMPORTANCE IN THE GLOBAL CLIMATE MARKET …....................................16

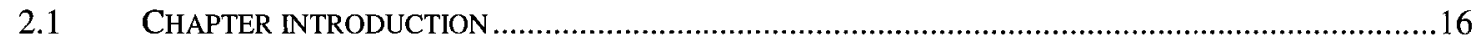

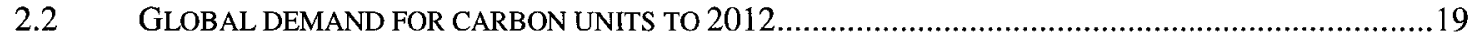

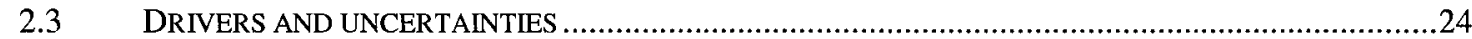

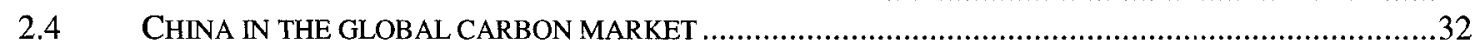

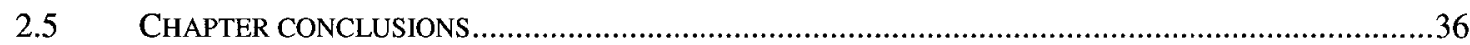

3 METHODS TO ESTABLISH A GHG BASELINE IN THE ELECTRICAL SECTOR .............39

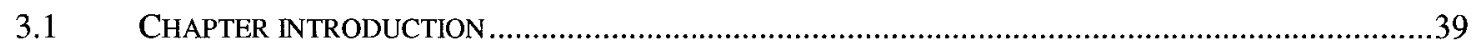

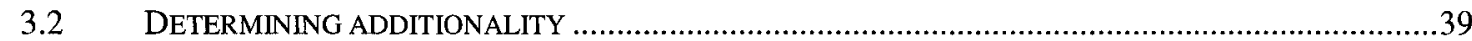

3.3 BASELINE STANDARDIZATION VERSUS PROJECT SPECIFIC BASELINE ANALYSIS............................44

3.4 A REVIEW OF MULTI PROJECT BASELINE METHODOLOGIES ........................................................45

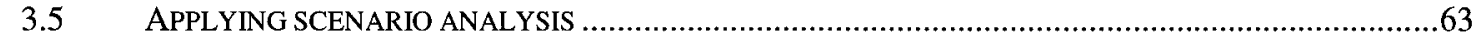

3.6 CRITERIA FOR ASSESSING BASELINE STRENGTHS AND WEAKNESSES .........................................70

4 USING A CURRENT PERSPECTIVE AS A BASIS FOR OPERATIONALIZATION..............75

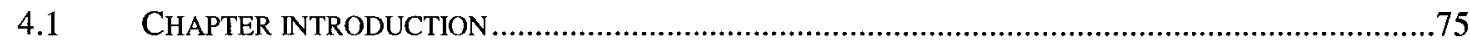

4.2 THE DEMAND AND SUPPLY OF ELECTRICITY IN CHINA ……....................................................75

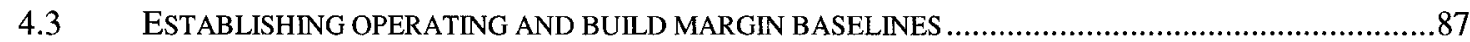

5 USING DECOMPOSITION ANALYSIS AS A BASIS FOR OPERATIONALIZATION ........95

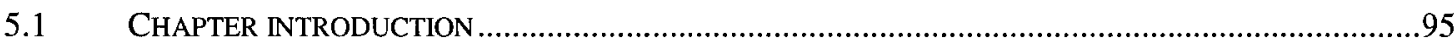

5.2 USING DECOMPOSITION IN THE ELECTRICITY DEMAND SECTOR..............................................96

5.3 USING DECOMPOSITION IN THE ELECTRICITY SUPPLY SECTOR ..........................................116

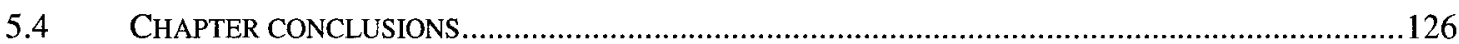

6 CHINA'S ELECTRICAL SECTOR INTO THE FUTURE .....................................................128

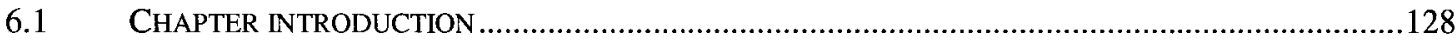

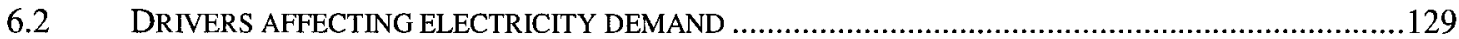

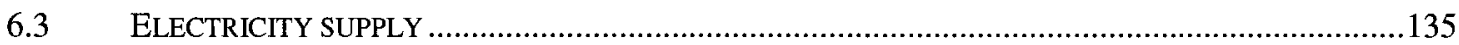

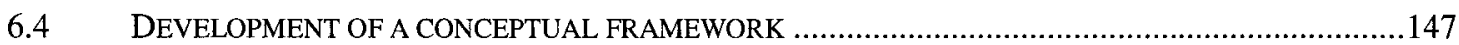

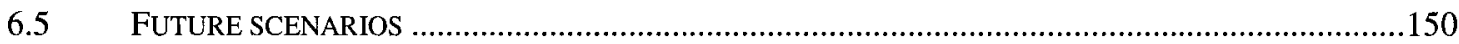

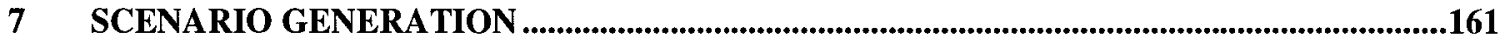

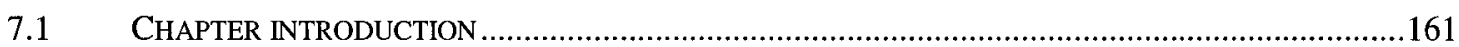

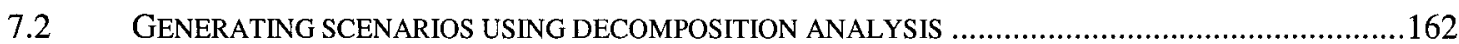

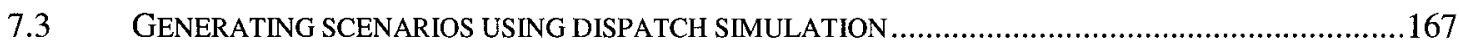

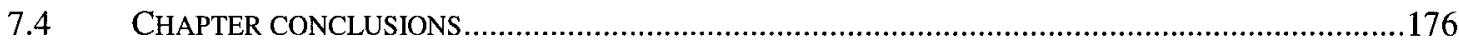

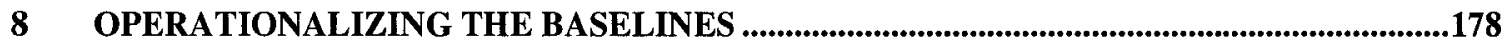

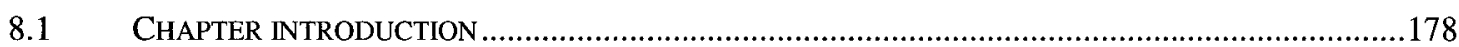

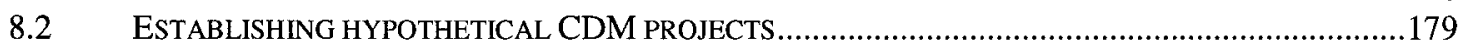

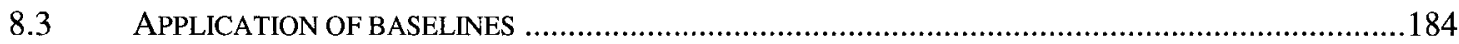

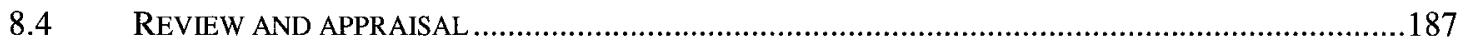

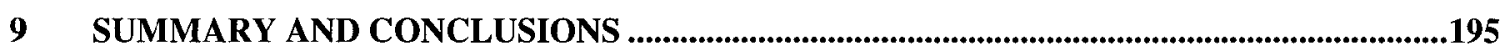

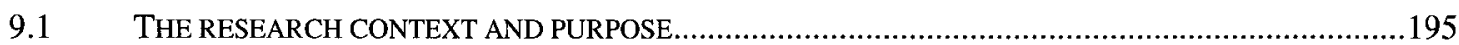

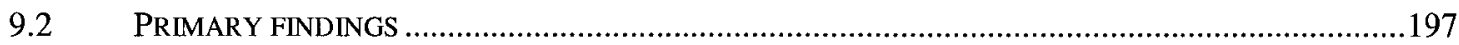

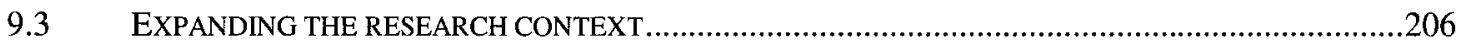


Table 1. Base year and projected 2010 GHG emissions (million metric tonnes) for all Annex I nations (derived from third national communications) ................................. 21

Table 2. Emission reduction potential from CDMs by economic sector in China (from

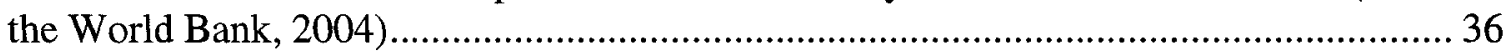

Table 3. Characteristics of different multi-project baseline methods ........................... 46

Table 4. Factors governing electricity demand and the emission characteristics of its

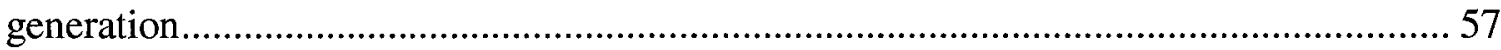

Table 5. Key criteria to evaluate CDM baselines (UNFCCC, 2001) ............................ 70

Table 6. Growth in household appliances in urban China: 1980 - 2002 (derived from

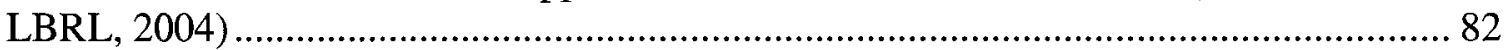

Table 7. Electricity generated by regional grid in China in 2005 (CSPIN, 2006) .......... 84

Table 8. Summary of statistical variation in provincial level emission baselines ..........91

Table 9. Summary of statistical variation in regional level emission baselines ..............93

Table 10. Empirical data used to study industrial sector electricity demand (data from

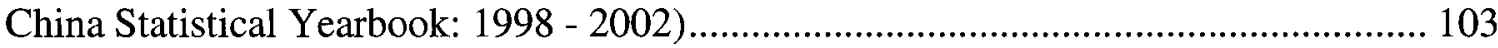

Table 11. Key trends influencing industrial sector electricity demand ........................ 108

Table 12. Factor contributions to changes in industrial sector electricity demand between

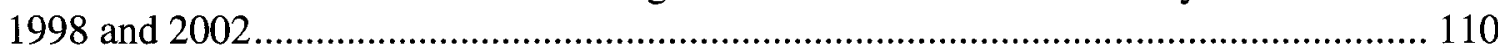

Table 13. Factor contributions (including changing fuel shares) on industrial sector

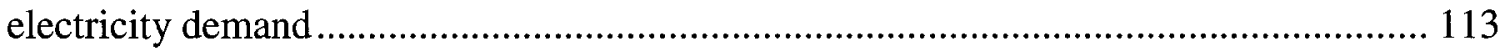

Table 14. Decision nodes affecting electricity demand in China ................................ 135

Table 15. Characteristics of sub-critical generation units versus clean coal technology144

Table 16. Decision nodes in the electricity supply sector....................................... 147

Table 17. General socio-economic and technological attributes of scenarios ............... 152

Table 18. $\mathrm{CO}_{2}$ emissions from electricity generation under different scenarios ........... 163

Table 19. Electricity generation by source by scenario as calculated by the IPM......... 172

Table 20. Technical details of hypothetical CDM case studies ................................. 179

Table 21. Summary of project type national circumstance and additionality................ 183

Table 22. Calculated $\mathrm{GHG}$ baselines $\left(\mathrm{kg} \mathrm{CO}_{2} / \mathrm{KWh}\right)$ and emission reductions (ktonnes

$\mathrm{CO}_{2}$ ) by baseline method and CDM project case study........................................ 185

Table 23. Comparing sub and super critical coal electricity generation ....................... 223 
Figures

Figure 1. A contextual history of strategies to deal with atmospheric issues of global

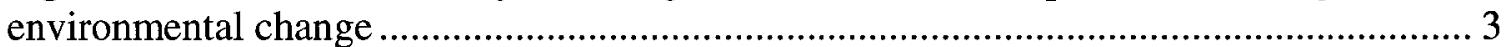

Figure 2. CDM Additionality Tool (from Ringius, 2004) ......................................... 42

Figure 3. Changes in electricity demand by sector (1980 and 2002) (LBRL, 2004)...... 78

Figure 4. Regional grid map of China (ICF, 2005) ................................................ 85

Figure 5. Operating and build margin baselines (provincial level). ........................... 89

Figure 6. Operating and build margins (regional level).............................................. 92

Figure 7. Structural and intensity impacts on industrial sector electricity demand (1998 -

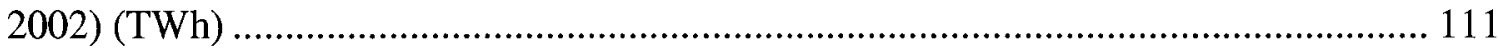

Figure 8. Impacts of changing fuel shares on industrial sector electricity demand (1998 -

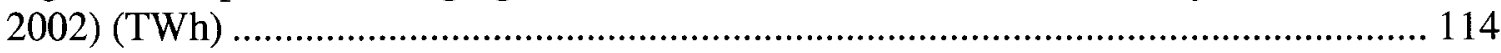

Figure 9. Decomposition of the carbon emission intensity of electricity generation .... 123

Figure 10. Trends in the energy efficiency of China's electrical sector....................... 124

Figure 11. Projected demand and supply of natural gas in China .............................. 140

Figure 12. A conceptual framework for modelling the development of China's electrical

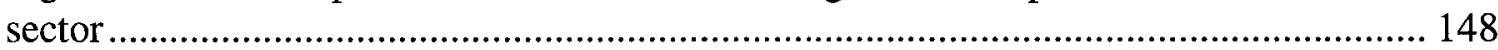

Figure 13. Two-dimensional framework for scenario setting (derived from the IPCC

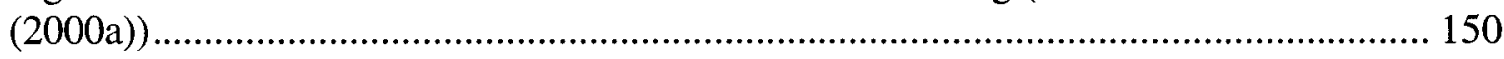

Figure 14. Placing the scenarios in a two-dimensional framework for scenario setting 153

Figure 15. Decomposition of carbon emission intensity - BAU scenario ..................... 164

Figure 16. Decomposition of carbon emission intensity - Conservative scenario......... 165

Figure 17. Decomposition of carbon emission intensity - Optimistic scenario .............. 166

Figure 18. Electricity generation by scenario in the IPM ...................................... 170

Figure 19. $\mathrm{CO}_{2}$ emissions by scenario in the IPM ........................................... 170

Figure 20. Emission intensity by region as calculated by the IPM - BAU scenario .... 174

Figure 21. Emission intensity by region as calculated by the IPM - Optimistic scenario

Figure 22. Emission intensity by region as calculated by the IPM - Conservative

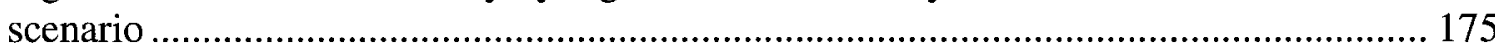

Figure 23. Geographical location of CDM project case studies .............................. 180 


\section{Appendices}

Appendix A: Detailed additionality assessment for potential electrical sector CDM projects in China

Appendix B: Sample Planning Design Document for a China electrical sector CDM project

Appendix C: Detailed tables showing contribution of different Chinese industrial subsectors to changes in electricity demand

Appendix D: Glossary (including definitions for acronyms used) 
Acronyms

AAU: Assigned amount unit

BAT: Best available technology

BAU: Business as usual

BCM: Billion cubic metres

BM: Build margin

CAD: Canadian dollar

CCBMC: China Coalbed Methane Clearing House

CDM: Clean Development Mechanism

CDM EB: Clean Development Mechanism Executive Board

CER: Certified emission reduction

CEPB: China Electric Power Yearbook

$\mathrm{CH}_{4}$ : Methane

CFC: Chlorofluorocarbon

CMM: Coal mine methane

$\mathrm{CO}_{2}$ : Carbon dioxide

COP: Conference of the Parties

$\mathrm{CM}$ : Combined margin

CPI: Consumer price index

CSP: China State Power

CSPIN: China State Power Information Network

CCT: Clean coal technology

DA: Dispatch analysis

DM: Dispatch model

EEA: European Environmental Agency

EIA: Energy Information Agency

EIT: Economy in transition

EPA: Environmental Protection Agency

ESPON: European Spatial Planning Observation Network

ETS: Emission trading scheme

EU: European Union

EU ETS: European Union Emission Trading Scheme

FDI: Foreign direct investment

GCC: Global climate change

GCSE: Grams of standard coal equivalent

GDP: Gross domestic product

GEC: Global environmental change

GERT: Greenhouse Gas Emissions Reduction Trading Program

GHG: Greenhouse gas

GJ: Gigajoule

HFC: Haloflourocarbon

IDA: Index Decomposition Analysis

IEA: International Energy Agency 
IISI: International Iron and Steel Institute

IPCC: Intergovernmental Panel on Climate Change

JI: Joint Implementation

KG: Kilogram

KP: Kyoto Protocol

Ktonne: Kilotonne

KW: Kilowatt

KWh: Kilowatt hour

IIASA: International Institute of Applied Systems Analysis

I-O: Input output

IRR: Internal rates of return

LBRL: Lawrence Berkeley Research Laboratory

LNG: Liquid natural gas

LULUCF: Land use, land use change, and forestry

MAC: Marginal abatement cost curve

MPB: Multi-project baseline

METI: Ministry of Economy, Trade, and Investment

$\mathrm{MTCO}_{2}$ : Million metric tonnes of carbon dioxide

$\mathrm{MTCO}_{2} \mathrm{e}$ : Million metric tonnes of carbon dioxide equivalent

Mtonne: Megatonne

MW: Megawatt

MWh: Megawatt hour

NDRC: National Development and Reform Commission

NG: Natural gas

NPV: Net present value

$\mathrm{N}_{2} 0$ : Nitrous oxide

OECD : Organization for Economic Cooperation and Development

OM: Operating margin

P: Primary

PDD: Project Design Document

PFC: Pleuroflourocarbon

PPP: Purchasing power parity

PRC: People's Republic of China

REL: Renewable Energy Law

RMU: Removal unit

SDA: Structural Decomposition Analysis

SH: Small hydro

SRES: Special Report on Emissions Scenarios

$\mathrm{SF}_{6}$ : Sulfur Hexafluoride

SOE: State Organized Enterprise

S-M: Secondary manufacturing

S-P: Secondary primary

T\&D: Transmission and distribution

TCM: Trillion cubic metres

TWh: Terawatt hour

UNFCCC: United Nations Framework Convention on Climate Change 
U.S.: United States

USD: United State's dollar

WB: World Bank

WEC: World Energy Council

WG: Wind generation

WTO: World Trade Organization 


\section{Introduction}

\subsection{Nature of problem}

Environment and society are increasingly becoming interconnected as human influences upon environmental processes have become so pervasive that they have started to contribute to global environment change (GEC) ${ }^{1}$ (Wilk, 2002). Anthropogenic climate change is a prominent example of GEC and involves drivers tied to changes in population size and demographic profile, economic activity, and technology. These drivers determine the provision and consumption of energy and the greenhouse gas (GHG) emissions that result from these, increasing atmospheric carbon concentrations that contribute to global climatic change by increasing the natural greenhouse effect (IPCC, 2000b). These interconnections make the human interface with the environment highly dynamic and complex, changing with the scale and characteristics of our socioeconomic activity.

Climate change is also an issue immersed in uncertainty and an issue with a very long temporal dimension, particularly when considering that it is not only humankind's present but also its future actions that will determine the progression of the climate change issue. Changes could occur in any of the drivers of GHG emissions and the level of GHG emissions humankind puts into the atmosphere (IPCC, 2000a). This presents a daunting challenge because in order to properly assuage the potential for climate change policy to reduce GHG emissions, an understanding must be gained of how these place in the context to possible emissions levels into the future (IPCC, 2001). Therefore,

\footnotetext{
${ }^{1}$ Acronyms are presented throughout and a dictionary of acronyms and other terminology is presented at the start of the dissertation.
} 
understanding the potential success of climate policy hinges upon having tools that can give insight on the consequences of mitigative options in a highly uncertain world (Grubb, 2001; Alcamo et al., 2002).

Partially due to the enormity of the climate change issue, its connections to such fundamental facets of human society and economy as energy consumption, and the uncertainties underlying its future progression, establishing policy to address this GEC has involved a long, tenuous, and politicized international effort. To illustrate this, international climate policy initiatives can be compared to actions to mitigate stratospheric ozone depletion (Munn et al., 1997), a GEC that involves a limited range of chemicals and has required a focused international policy response (Powell, 2002) (see figure 1). 
Figure 1. A contextual history of strategies to deal with atmospheric issues of global environmental change

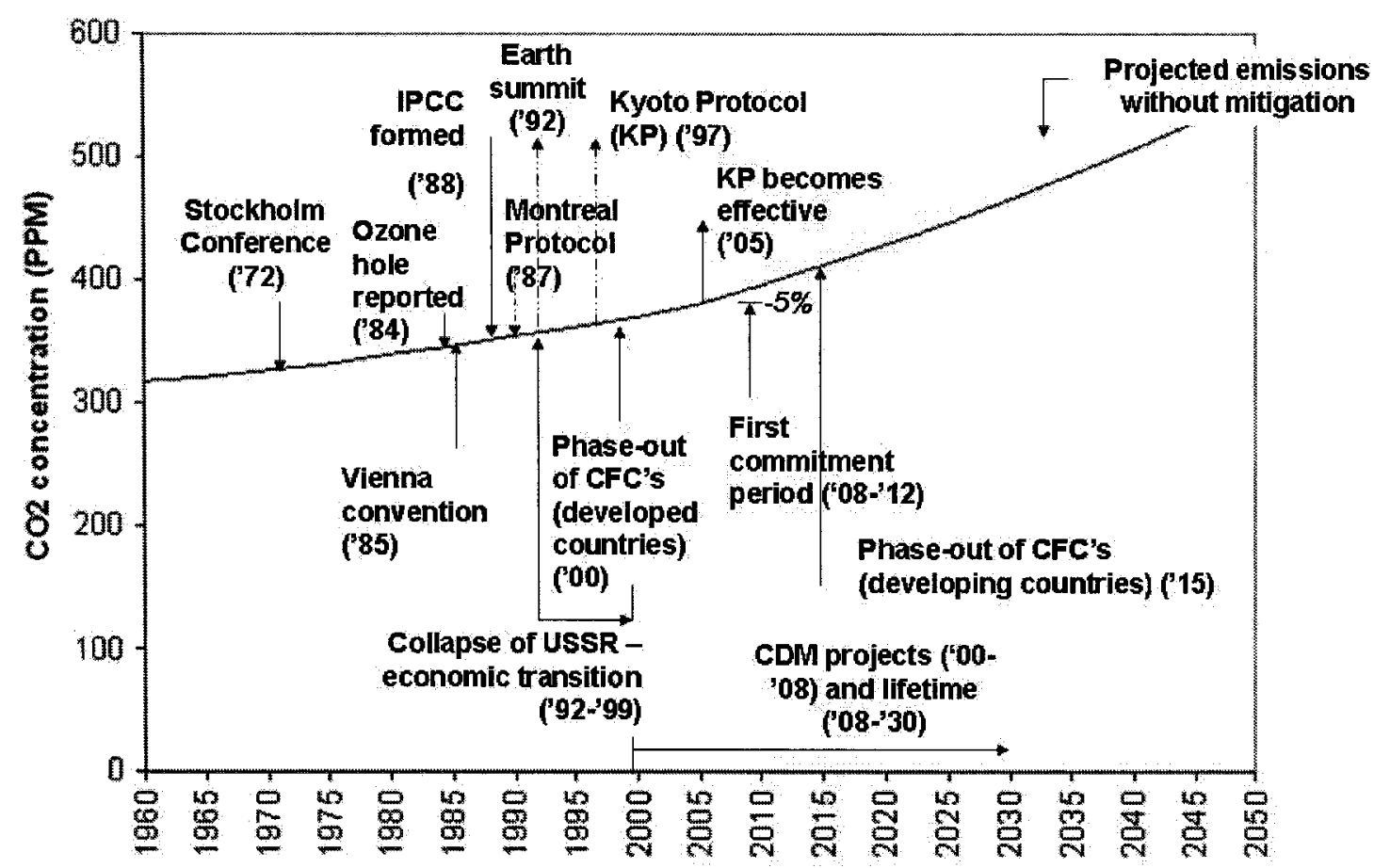

The groundwork for global environmental awareness around each of these environmental challenges began at the United Nations Conference on Human Environment in Stockholm Sweden (known as the 'Stockholm Conference') in 1972 as here the international community first came together to discuss global environmental concerns (Seyfang, 2003). After the scientific community increased the understanding of these particular issues during the next decade, a global consensus and mitigation plan began to emerge, primarily during the Vienna Convention in 1985 concerning the protection of the ozone layer, the Montreal Protocol enacted in 1987 concerning action on the ozone depletion issue (Powell, 2002), and the establishment of the Intergovernmental Panel on Climate Change (IPCC) for the climate change issue in 1988 (IPCC, 2004). For both 
issues, negotiations continued thereafter, but for ozone depletion, the speed of action was swift. A plan was enacted to eliminate emissions of chlorofluorocarbons (CFC), the main contributor to ozone depletion, from developed nations by 2000 , with developing nations given to 2015 to do the same (Powell, 2002). The same, however, cannot be said of the global climate change issue. Only a five percent reduction in global GHG emissions can be expected by the first commitment period that expires in 2012, while formal negotiations beyond the first commitment period only commenced during the $11^{\text {th }}$ Conference of the Parties (COP-11) in Montreal. Major Parties to the United Nations Framework Convention on Climate Change (UNFCCC) continue to express concern about the science of global climate change (GCC) and hence a global solution remains elusive. The UNFCCC entered into force in 1994, and now has 189 Parties that have ratified it.

A central reason for the slower response to the anthropogenic climate change issue is that this has direct linkages with one of the fundamental requirements for human economic activity: GHG emissions associated with the consumption of energy. Many of the approaches to mitigate GHG emissions are therefore broad in dimension and seek to embrace multiple facets of the economy-environment interface. For example, economic instruments, particularly market-based mechanisms such as international emissions trading or linking emission reduction efforts with the investment of clean energy technology in developing and industrializing countries bring together new energy production technologies, tax incentives, and international environmental regimes to address human-related causes of climate change (Kohn, 1996). 
At the heart of the global effort to mitigate emissions of GHGs is the Kyoto Protocol, an addition to the UNFCCC. The UNFCCC was signed in 1992 at the Earth Summit, and set forth a framework for action aimed at stabilizing atmospheric concentrations of GHGs in order to avoid "dangerous anthropogenic interference" with the climate system (as reported in Hansen (2005)). The framework of the UNFCCC thus builds upon the findings of the IPCC, formed in 1988 by the World Meteorological Organization to study the issue of global climate change. The IPCC was established as scientists and policymakers from around the world began to grow more concerned with global increases in temperature and scientific evidence indicating that anthropogenic emissions of GHGs were a major contributor to this warming.

Under the UNFCCC, countries were divided into those with emission reduction commitment targets, including either countries with developed market economies or those on a transitional path towards a market economy (known as 'economies in transition', or EIT), and countries who can be considered to be still 'developing'. The former of these are listed in Annex I of the UNFCCC and are therefore interchangeably referred to as 'Annex I nations', while those countries not in Annex I are referred to as 'non Annex I nations'. How to bring developing nations into longer-term GHG reduction plans also remains a divisive issue amongst Parties to the UNFCCC. Seeing the long-term nature of the climatic change issue, this will become of increasing importance as GHG emissions from developing countries are growing the fastest.

During the first COP meeting in 1995 in Berlin, Germany, a mandate was issued calling on international cooperation in the reduction of global GHGs. However, it was only by the COP-3 in Kyoto, Japan, where a consensus on how to approach the climate 
change issue emerged: the Kyoto Protocol (KP). Here, the Parties agreed that emissions of Annex I nations in the entirety would have to be reduced by an average of $5.2 \%$ below 1990 levels by the 'first commitment period' that spans 5 years from 2008 to 2012 . The Protocol legally entered into force 8 years after its initial formation when Russia confirmed its ratification in November 2004. As of April 2006, 163 Parties have now ratified the Kyoto Protocol, representing nearly $62 \%$ of all Annex I emissions.

The negotiations during and after the formation of the Kyoto Protocol created a number of tools and mechanisms meant to lay the groundwork to bring developing nations into the international framework of emission reductions. For example, the Clean Development Mechanism (CDM) is a flexibility mechanism, designed to create economic efficiencies through international trade and also to encourage and stimulate sustainable development practices in developing nations. The CDM operates by allowing governments and corporate entities of Annex I nations or non-Annex I nations themselves to make investments in non-Annex I nations that will lead to lower GHG emissions and encourage sustainable development through the transfer of cleaner, advanced technology or more efficiency industrial processes. Article 12 of the Protocol summarizes the goals of the CDM as:

- To assist Annex I Parties (countries and corporate entities) in achieving compliance with their quantified emissions limitation and reduction commitments.

- To assist countries not included in Annex I in achieving sustainable development and in contributing to the ultimate objectives of the Framework Convention 
The analysis completed is largely focused on the first of these goals since baselines pertain to the reduction of GHG emissions and not specifically to the promotion of sustainable development. Nonetheless, sustainable development is considered to a degree through the assessment of the additionality of the hypothetical CDM projects proposed since projects can only be deemed non-additional if they consist of a project that involves new and advanced technological or operational processes.

In November 2005, the eleventh Conference of the Parties took place in Montreal, Canada. Over forty decisions were made at the conference, six relating specifically to the CDM. Particularly, decisions were made concerning the definition of eligible projects for simplified modalities, forestation projects, projects to capture carbon, governance of the $\mathrm{CDM}$, and compensation for work concerning the CDM. The first of these areas is the only area that has direct relevance to this dissertation as decisions were made stipulating that electrical sector projects equal or less to $15 \mathrm{MWe}$ in capacity or demand side projects reducing $15 \mathrm{GWh}$ or less of electricity per year could used simplified methods to calculate the emission baseline used. As all of the CDM project activity considered in this research exceeds this threshold, this decision does not apply to the baseline analysis completed.

The nature of the CDM requires that the environmental integrity of the Protocol be ensured as emission credits are based upon a CDM project's future capacity to reduce emissions. This involves a 2-step accounting process to assess the environmental integrity of CDM projects. The first step involves estimating the business as usual (BAU) scenario, a baseline into the future against which future GHG emission reductions can be calculated. The 2 nd step then estimates GHG reductions that can be directly 
attributed to the implementation of a CDM project or projects. A comparison of the GHG emissions under the two "plausible futures" (BAU vs. CDM) isolates the GHG reductions stemming directly from the CDM. The difference between these two is then described as the "additionality" of the CDM project. These two steps are imperative for a project to be both successfully approved by the CDM Executive Board, the regulatory body governing the mechanism, and to meet goals of GHG reductions specified in the Protocol. However, while this 2-step procedure for estimating CDM additionality can be described in relatively simple terms, it is in fact a very complex and contested process that involves considerable uncertainty over long periods of time (i.e. several decades). The operating life span of CDM projects in the electrical sector is typically +30 years, during which time major economic and political shifts can be expected. In addition, there are several methods to estimate future baselines and the effect of the CDM on future emissions.

The complexities of implementing CDMs and estimating CDM impacts on future GHG emissions are well illustrated in the People's Republic of China (PRC). ${ }^{2}$ This is particularly the case with electricity generation as this represents a key economic sector that plays an increasingly important role in China's development and in the global environment. Due to the rapid growth in its economy, electricity demand is accelerating. Coal continues as the dominant fuel of choice for electricity generation and thereby leading electricity generation to have a devastating impact on local, regional and the global environment through the many air pollution consequences of coal combustion. This sector also presents an excellent example of a dynamic system with many

\footnotetext{
${ }^{2}$ Taiwan has not been included as part of the PRC in this dissertation. From this point forward, China is used in reference to the UNFCCC.
} 
uncertainties about its future development, and therefore, a system that can help emphasize the strengths and weaknesses of different baseline approaches. Seeing its contribution to China's domestic economy and to the global environment, China's electrical sector will likely attract significant CDM project activity into the future, but the potential for CDMs to reduce China's future GHG emissions remains understudied.

\subsection{Purpose and research objectives}

The purpose of this dissertation is to operationalize selected analytical methods for establishing the emission reduction capacity of potential CDM projects in China's electrical sector in order to illustrate the strengths and weaknesses of these methods. Three differing methodological approaches are selected, with each taking a different perspective of how to estimate future additionality: a) the use of historical analysis in order to help predict the future b) using current characteristics of the sector as indicators of the future $\mathrm{c}$ ) and methods based on the mathematical simulation of future events. Overall, five central questions are embedded in this research:

1) What is China's strategic importance generally and in particular China's electrical sector in the global climate change regime?

2) Can (and how) does a historical perspective of China's electrical sector give indication of what might occur in the future in terms of GHG emissions?

3) Can (and how) does the current circumstance of China's electrical sector give indication of what might occur in the future in terms of GHG emissions?

4) Can (and how) does a simulation of China's electrical sector's future development give indication of what might occur in the future in terms of GHG emissions? 
5) What lessons can be learned from the operationalization of the selected baseline methods?

Within the three different general methods, four techniques for baseline formation have been identified.

The first and second baseline methods are derived from technology detailed bottom-up attributes of electricity generation for the most recent years available and include the 'operating margin' (OM) and 'build margin' (BM) methods. The operating margin method considers the technical details of the entire electrical system, while the build margin approach considers only the most recently constructed plants. These are examples of using the current characteristics of the electrical sector as an indicator of the future. In addition to being relatively straightforward and simple approaches, operating and build margins are also baseline techniques suggested for use by the UNFCCC if only general level statistics are available for the sector. The third technique considered, 'factor decomposition', is a technique based upon the analysis of historical data to determine the contribution of factors that have influenced the emission intensity of electricity generated, with these factors then studied to see how they might change into the future. It is currently not a baseline technique suggested for use by the CDM Executive Board (CDM EB) ${ }^{3}$, although it can be found throughout the energy and environment literature for similar studies. The fourth technique considered is dispatch simulation. This is a method based upon a more complex, mathematical simulation

\footnotetext{
${ }^{3}$ The CDM EB was established by the UNFCCC to help govern the CDM. The CDM EB is made up of 10 members - one member from each of the five United Nations regional groups, two other members from the Parties included in Annex I, two other members from the Parties not included in Annex I, and one representative of the small island developing States.
} 
approach that considers the electrical sector as a dynamic demand-supply system where future development is projected based upon a series of assumptions about the future. It also is the method of preferred choice by the UNFCCC as it is considered more accurate than other options.

There are two remaining points. First, rather than simply focusing upon the first commitment period (which spans from 2008 to 2012) as most baseline analyses do, this assessment considers baselines to the year 2020. This is important for a number of reasons. As shown in figure 1, most CDM projects in the electrical sector typically have a 30 to 40 year lifespan or longer. Their existence could coincide with such economic shocks as those that occurred in the former Soviet Union between 1992 and 1999. Between these years, the economic output of the various Soviet states declined by 40 percent or more and when energy intensities rose sharply. The life span of electrical generating technology means that the additionality of related CDM projects is dependent on the future development of the energy systems in which these are implemented. This being, concerned decision makers and stakeholders must have suitable tools that they can use to measure the longer-term environmental impacts of such projects. Secondly, rather than using only one BAU scenario as most baselines do, three general scenarios are developed. This is important as using multiple scenarios can help identify the strengths or weaknesses of different baseline approaches in handling a key criterion - the measurement of uncertainty. It is also important since using different scenarios can help demonstrate how the environmental additionality criterion for CDM projects might be affected under different routes of development. 
Given future uncertainties underlying economic processes such as electricity demand and generation, and given the various approaches for estimating CDM additionality, it should not be surprising that the implementation of CDM projects under the KP has been hampered by underdeveloped methods for estimating future baselines and the potential benefits of CDM projects.

\subsection{Dissertation scope}

The scope of this dissertation is restricted to the review and appraisal of an operationalization of baseline methodologies using China as a case study. Although limited to this one dimension of the CDM, this is only one of many intersecting issues and relevant questions concerning the CDM and its implementation. It is important to highlight these other dimensions in order to help situate the work undertaken within the broader field of research and research inquiries that exists for the implementation of the CDM.

One significant area of inquiry concerning the CDM is the extent that transaction costs associated with, amongst other requirements, baseline calculation, project registration, and administrative expenses discourages the implementation of potential CDM projects. Currently there is a reliance on often expensive analyses and complicated registration and administrative procedures for potential CDM projects (Michaelowa et al., 2003; Michaelowa et al., 2005). This is especially the case with small scale CDM projects given that small projects have relatively more transaction costs in relation to their revenues. Jotzo and Michaelowa (2002) illustrate through the use of a general equilibrium model that only a minor change in transaction costs could impact on the volume of potential GHG emission reductions from CDM projects globally by $-/+80$ 
$\mathrm{MTCO}_{2} \mathrm{e}$, while Michaelowa et al. (2005) later proposed that transaction action costs limit the economic viability of projects equal to or less than $0.20 \mathrm{MTCO}_{2} \mathrm{e}$ of emissions per year. This proposition was further investigated through a review of CDM project activity in India by Krey (2005) who concluded that the transaction costs associated with these CDM projects were a significant barrier for their implementation. An additional limiting factor for the broader implementation of the CDM are the institutional barriers that also must be overcome (Del Rio, ; Michaelowa et al., 2005). In particular, host countries for CDM projects must establish institutional bodies to help administer and monitor the CDM projects that take place within their territory. Some countries have indicated that they will levy additional fees to help offset these costs, while other countries have indicated that they will only establish these institutions when the price of carbon reaches a certain level.

In order to reduce the transaction costs and administrative barriers of potential CDM projects, the UNFCCC has established different processes and guidelines to help streamline the CDM process, some of which have been mentioned previously concerning discussions that took place at COP-11 in Montreal in February 2006. However, how to improve the CDM process so that there is a balance between meeting the environmental and sustainable development objectives of the mechanism while also ensuring the highest volume of emission reductions remains a significant area of discussion in the literature and a question that remains to be fully resolved. Similarly, the full impact of the institutional barriers of the mechanism in regards to its implementation also remains unclear. 
Clearly, the operationalization of specific CDM projects involves a complex and multi-dimensional set of activities. Key issues pertain to establishing baseline analyses and ensuring that these and other transaction costs do not discourage potential CDM projects, while also ensuring that institutional barriers do not limit project implementation. Each of these issues contains legitimate concerns in their own right while also having strong synergies. This research contributes primarily to establishing and analyzing CDM baselines and offers suggestions to foster improved linkages between baseline analyses and related CDM issues.

\subsection{Structure of dissertation}

Nine chapters are included. Each chapter forms a foundational basis for material and analysis in subsequent sections and therefore each plays an important role in reaching the overarching objectives of the research. Chapter 2 gives the context of the study, first introducing the global carbon market, then China's electrical sector and how this places within the global carbon market. Chapter 3 reviews major methodological issues as well as developing necessary material to be used in the later analysis. Important features of this chapter include an overview of the baseline and scenario generation methods used. Chapter 4 then presents an historical overview of the electrical sector, culminating in the establishment of the first set of CDM baselines (the operating and build margin approaches). In chapter 5, decomposition analysis is then undertaken, with components of this also used in the scenario analysis presented in later chapters. Chapter 6 starts to lay the groundwork for the analysis of the electrical sector into the future, culminating in the building of a conceptual model for the actual modelling of the future and the envisioning of three storylines. In chapter 7 , three scenarios are then generated from 
these storylines using the appropriate baseline methodologies. Chapter 8 follows by reviewing and appraising the three constructed baselines through the application of 5 hypothetical CDM project case studies established to allow actual calculation of the emission reductions achieved using the different baseline methods. Chapter 9 concludes.

Each chapter includes a synopsis that provides a snapshot of the chapter and its role within the overall dissertation. Appendix A includes a description of the additionality assessment for the CDM project case studies developed, Appendix B an example of a Project Design Document (PDD) for a project case study, Appendix C detailed tables showing the contribution of different industrial sub-sectors in China to changes in electricity demand, and Appendix D a glossary. 


\section{China's importance in the global climate market}

Synopsis: China's electrical sector is put in the context of the global carbon market, where specifically it is asked why a focus on China is important. To develop a better understanding of the carbon market and China's place within this, national emission projections found in the third national communications to the UNFCCC are compared to national emission targets established under the Kyoto Protocol. This illustrates that under current policies emissions will likely exceed Protocol targets, but that Russia's available 'hot air' surpasses the total possible demand for emission credits in the market. Nonetheless, there is significant demand for emission reductions from Clean Development Mechanism and Joint Implementation projects. These largely originate from the European Emission Trading Scheme and government purchases. China is then considered as both a major GHG emitter and major provider of emission reduction possibilities. Here it is identified that it is in the electrical sector where the most economical and plentiful emission reductions exist.

\subsection{Chapter introduction}

On February 16th 2005 the Kyoto Protocol to the UNFCCC entered into force with the overarching objective to reduce GHG emissions of Annex I Parties ${ }^{4}$ by an annual average of 5.2\% over the five years from 2008 to 2012 (the first commitment period) from the 1990 base year. Quantified emission reduction requirements were made for a basket of six GHGs $\left(\mathrm{CO}_{2}, \mathrm{CH}_{4}, \mathrm{~N}_{2} \mathrm{O}, \mathrm{SF}_{6}, \mathrm{PFCs}\right.$, and $\left.\mathrm{HFCs}\right)$, ranging from $8 \%$ reduction to a $10 \%$

\footnotetext{
${ }^{4}$ Annex I Parties are countries with emission targets for the period 2008-12 as per Article 3 and Annex B of the Kyoto Protocol and include the 24 original OECD members, the European Union, and fourteen countries with economies in transition.
} 
increase from the base year for each party (for the majority this is 1990). As a part of ratification, the UNFCCC requires all Parties to submit a national communications containing a detailed emission inventory, the national context of the activity leading to emissions, measures to reduce emissions, and GHG projections that take into account these different factors. Three national communications have been submitted to the UNFCCC by most Parties to the Convention, and by January 2006, all Annex I Parties were expected to have submitted their fourth.

This chapter seeks to better understand China's role in the context of the expected size and structure of the global carbon market, defined as the demand for and supply of emission reductions over the first commitment period. This is accomplished by first summarizing the emission projections found in the third national communications, second, examining significant economic, political and regulatory drivers that are impacting the demand for emission reductions over the first reporting period, third, reviewing the most recent trading activity of emission reduction opportunities and placing these occurrences in the context of changes in the aforementioned drivers, and finally, assessing China's role in the overall carbon market.

\subsubsection{The Kyoto Protocol}

The Kyoto Protocol of 1997 was a significant addition to the initial Framework Convention as it set individual, legally-binding emission reduction targets for each party listed in Annex I of the Convention. In order to comply with its emissions target, a maximum amount of emissions that a Party could emit over the commitment period was established, with the total amount referred to as a Party's available assigned amount units (AAUs). 
The Kyoto Protocol also set forth a number of methods for Annex I Parties to reduce emissions, ranging from domestic actions to the use of three unique international flexibility mechanisms, including emission trading of AAUs between Annex I nations, the CDM, and Joint Implementation (JI). The CDM works by allowing both Annex I and non-Annex I nations (or any legal entities thereof) to invest in non-Annex I nations to reduce emissions from an established baseline, resulting in the creation of certified emission reduction (CERs) units. JI, alternatively, refers to a developed country receiving emissions reduction units (ERUs) by that country financing projects that reduce net GHG emissions in another developed country. Typically, the recipient country is an EIT. In 2001, the Marrakech Accords established a further mechanism involving the GHGs removed from the atmosphere through eligible sink activities in the land use, landuse change and forestry (LULUCF) sectors. These activities generate credits known as removal units (RMUs). At the end of the first commitment period, a computerized system of registries will ensure that Annex I Parties are in compliance with their emissions targets by allowing the comparison of each Parties' emissions during the commitment period with their holdings of AAUs, ERUs, CERs, and RMUs (UNFCCC, 2005).

The Marrakech Accords also set out detailed rules how the mechanisms within the Protocol would operate. Since this time, there have been a number of other significant developments affecting the emerging global carbon market, with perhaps the most significant event occurring at the start of 2005 when the European Emissions Trading Scheme (ETS) entered into force. This is a regional trading scheme in Europe that is uniquely linked with the larger Kyoto market through the use of transferable credits. 
Throughout the chapter, ERUs, CERs, AAUs and RMUs are collectively referred to as 'carbon units'.

\subsection{Global demand for carbon units to 2012}

\subsubsection{Projections in the third national communications}

Utilizing a three step process, aggregate demand for Kyoto units is first constructed from the third national communications of the Annex I nations as available from the UNFCCC website (UNFCCC, 2006a). The first task is to compile base year emissions for each respective country - available from the GHG database on the UNFCCC website (UNFCCC, 2006b). The second task is to determine the emission reduction requirements of Parties to the Convention (as available in Annex B to the Kyoto Protocol). This allows the determination of national AAUs. The final task is to determine emission projections to the year 2010 for each Annex I nation as provided either in their national communications or through extrapolation. The majority of the Annex I countries have now submitted their third national communications, with Russia's being the latest to arrive in July 2003. Therefore, the figures provided in this chapter consider the most recent information available for each country. At the time of writing, the countries that had not submitted a third national communication included the Ukraine, Belarus, Croatia, Lithuania, Luxembourg, Romania, and Slovenia. For these, earlier national communications were used to provide emission projections for 2010 .

The majority of countries provide two emission scenarios: emission projections structured upon current or policies being implemented in the near future (termed 'with measures' in the national communications), and emission projections structured upon a 
fuller set of national climate policies that could be implemented into the future (termed 'with additional measures' in the national communications). The exception is the United States and Canada who do not provide multiple emission projections.

The emission projections of the respective Annex I counties are aggregated and presented for eight world regions in order to allow comparison and analysis of general trends occurring in the global carbon market (see table 1). These include OECD Oceania (comprised of Australia and New Zealand), the total European region (EU total comprised of the initial member countries of the EU (EU), the accession countries to the EU since 2004 (EU - ascending) and Germany, Japan, Russia, the Ukraine, the United States, and Canada. The last six countries are shown separately as each plays a unique role in the global carbon market: Japan, the United States and Canada as contributors to emissions above their AAU, Germany, Russia and the Ukraine due to their lowered emissions from their AAU. 
Table 1. Base year and projected $2010 \mathrm{GHG}$ emissions (million metric tonnes) for all Annex I nations (derived from third national communications)

\begin{tabular}{|c|c|c|c|c|c|c|}
\hline \multirow[b]{3}{*}{ Reqion } & \multirow[b]{3}{*}{$\begin{array}{l}\text { Assigned } \\
\text { amount } \\
\text { units }\end{array}$} & \multirow{2}{*}{\multicolumn{3}{|c|}{ With Measures }} & \multicolumn{2}{|c|}{ With Additional } \\
\hline & & & & & \multicolumn{2}{|c|}{ Measures } \\
\hline & & 1990 & 2010 & $\begin{array}{c}\text { Above/ } \\
\text { below } \\
\text { AAU }\end{array}$ & 2010 & $\begin{array}{c}\text { Abovel } \\
\text { below } \\
\text { AAU }\end{array}$ \\
\hline & \multicolumn{6}{|c|}{ Million metric tonnes of carbon dioxide equivalent } \\
\hline United States & 5,702 & 6,131 & 8,166 & 2,464 & 8,166 & 2,464 \\
\hline Canada & 571 & 607 & 770 & 199 & 770 & 199 \\
\hline Japan & 1,172 & 1,247 & 1,317 & 145 & 1,218 & 46 \\
\hline OECD Oceania & 531 & 497 & 757 & 226 & 638 & 106 \\
\hline EU (including UK) & 3,016 & 3,102 & 3,304 & 288 & 3,010 & -6 \\
\hline EU - Ascending & 1,422 & 1,530 & 1,284 & -138 & 1,223 & -199 \\
\hline Germany & 966 & 1,223 & 812 & -154 & 800 & -166 \\
\hline Europe region & 5,404 & 5,855 & 5,400 & -5 & 5,034 & -371 \\
\hline Ukraine & 919 & 919 & 783 & -136 & 694 & -225 \\
\hline Russia & 3,040 & 3,040 & 2,432 & -608 & 1,976 & $-1,064$ \\
\hline \multicolumn{7}{|l|}{ Total emissons } \\
\hline With U.S. & 17,339 & 18,296 & 19,626 & 2,286 & 18,496 & 1,156 \\
\hline Without U.S. & 11,638 & 12,165 & 11,460 & -178 & 10,330 & $-1,308$ \\
\hline \multicolumn{2}{|c|}{ Kyoto unit demand - no U.S. } & & & 566 & & $2=-19$ \\
\hline Russian 'hot air' & & & & -744 & & $-1,289$ \\
\hline
\end{tabular}

Under the 'with measures' emission trajectory, emissions are 2,286 million metric tonnes of carbon dioxide equivalent $\left(\mathrm{MTCO}_{2} \mathrm{e}\right)$ above the total assigned AAUs in 2010 when including the United States, but $178 \mathrm{MTCO}_{2} \mathrm{e}$ below when not. By 2010 , the United States will have emissions that are over $40 \%$ above their Kyoto target. Considering the size of its economy, this means that U.S. participation dominates the demand side of the market. The other three countries with emissions above their AAUs are Canada, Japan and Australia. Although Australia has not formally ratified the Protocol, it has agreed in principal to its objectives and is actively trying to reach its requirements under the treaty. Under this emissions trajectory the total Kyoto unit demand, or the total emission balance when not considering the tradable AAU's of Russia and the Ukraine, is projected to equal 
$566 \mathrm{MTCO}_{2} \mathrm{e}$ in 2010 and, assuming linearity, 2,830 $\mathrm{MTCO}_{2} \mathrm{e}$ between 2008 and 2012. Based on the most recent projections available from Russia and the Ukraine however, the available AAU from the EIT will exceed this amount. Nonetheless, even if there are AAUs available from the EIT in excess of the net Kyoto unit demand, for a variety of political and economic reasons, there is a significant market developing for project-based emission reduction credits.

The emission trends of the different Annex I countries reflect the economic, technological and political circumstance of each region. When considering the countries with emissions above their AAU, it is useful to consider the decomposition analysis of Hamilton and Turton (2002) who study the emission trends of all OECD nations from 1987 to 1997 . For the U.S. they observed that although the country was able to decrease its energy intensity faster than any other country, the largest contributing factor to its increase in emissions were changes in energy use to more carbon intensive fuels, predominantly in the electrical sector, and the growth in its economy. In Canada, energy intensity decreases were smaller, and this was compounded by unfavorable changes in fuel shares. The primary reason explaining Japan's increased emissions, with economic growth minimal and even recessionary over this time, were increases in its energy intensity. This was because large investments had already been made to increase energy efficiency after the oil shocks of the 1970's and due to the minimal financial resources available for capital turnover in the late 1980's and throughout the 1990's.

The projections until 2010 in the 'with measures' scenario largely reflect what has happened in these economies in the recent past, namely - minimal increases in energy efficiency in Japan, minimal reductions in the carbon intensity of the energy supply in all 
nations, and a growth in the fossil fuel sector in Canada. Under this scenario, the EU, in its aggregate, is within $3 \%$ of its Kyoto target. Recent observations showed that total GHG emissions in the EU-25 (without land use change and forestry) decreased by more than $5 \%$ from 1990 to 2003 , while in the EU-15 emissions decreased by nearly $2 \%$ over the same period (EU-Commission, 2006), so this projection may be in the high range. Although the EU has the most proactive climate change policies amongst the various Parties to the Convention, its success in limiting emissions has come about also from a number of non-climate change policies and processes. This includes the reunification of Germany (from 1990 to $1995 \mathrm{CO}_{2}$ emissions from the former East Germany fell by $44 \%$ ), the closure of a number of coal mining operations (totaling a reduction of approximately $30 \mathrm{MTCO}_{2} \mathrm{e}$ ), and shifts towards nuclear power in the United Kingdom (METI, 2004).

Examining now the Parties with emissions below their targets established under the Protocol, there are three of particular importance and interest, two of which (Russia and the Ukraine) are classified as EIT and therefore can trade their surplus AAU's, and the other being Germany. Over the past 15 years, due in part to the reunification with East Germany but also due to prolonged weakness in the economy and to improvements in both its energy intensity and fuel mix, Germany has seen a significant decrease in its aggregate emissions. In 2003, its emissions were approximately $18 \%$ below the 1990 base year (EEA, 2003). Russia and the Ukraine, alternatively, can attribute nearly all of their emission reductions to depressed economic activity since the collapse of the Soviet Union. The emission reduction projections from these latter two countries equal nearly 
$900 \mathrm{MTCO}_{2} \mathrm{e}$ under the 'with measures' emission trajectory, and present the main source of competition to the various Kyoto units.

Finally considered is the 'with additional measures' emissions trajectory. Generally, this emission trajectory considers the planned but not implemented emission reduction policy initiatives in the EU and other non-North American countries, in conjunction with the top range of AAU surpluses in the Russian and Ukrainian markets. Thus, this emission trajectory in its aggregate is that which will occur if the most optimistic economic and environmental policy measures are successful in Europe, Russia, and the Pacific, a combination of factors which make such a scenario less conservative that the 'with measures' scenario. If all Parties are included under such an emissions scenario, total emissions are $1,156 \mathrm{MTCO}_{2} \mathrm{e}$ above the aggregate AAUs of the Annex I countries. However, excluding the United States, total Party emissions are 1,308 $\mathrm{MTCO}_{2} \mathrm{e}$ below the requirements of the Protocol. Here, the EU in its entirety is able to meet its Kyoto targets, whilst Canada and the OECD Pacific, including Japan, are not.

\subsection{Drivers and uncertainties}

Factors influencing the demand and supply of Kyoto units are considered. The first is the impact of the U.S. as a non-player in the global carbon market, while the second and third factors considered are the emission reduction purchasing activities of other national governments of Parties to the UNFCCC, and corporate purchasing activity related to the EU ETS and non-EU corporate entities. Considered next are issues related to the supply of global carbon emission reductions, including the role and scale of allowances entering the market from Russia (the largest EIT and provider of surplus AAUs) and issues related to carbon mitigation projects in the developing nations and Annex I nations. In 
September 2004, Russia's President Putin announced that the country would ratify the Protocol, and this was passed by the State Duma in October 2004. Therefore, the primary role of Russia's allowances is whether the country will sell the units in the 2008-2012 period or retain these for future use.

\subsubsection{Impacts of the withdrawal of the United States}

In 2001, the U.S. administration officially withdrew from the Kyoto Protocol, citing that the agreement would negatively affect domestic economic growth and decrease competitiveness with industrializing nations (Christiansen, 2003). The results of this are that demand for Kyoto units has been lowered by nearly $80 \%$ (as indicated in table 1), with this decreasing the price for all Kyoto units as a result (Manne et al., 2004). The lowered price of carbon units is emerging now as a disincentive for countries implementing emission reduction initiatives domestically. Analysis by den Elzen and de Moor (2002), for example, showed that if the U.S. were a participant in the Kyoto process the total costs for Annex I countries to meet the requirements of the Kyoto Protocol would approach 19 billion USD in 2010, with over half of abatement efforts taking place domestically. However, by the U.S. withdrawing from the Kyoto Protocol, the projected costs of implementation drop to 3.5 billion USD. If assuming participants use the least cost route to emission reduction, the proportional demand for Kyoto units rises dramatically at the expense of domestic action. This would mean domestic action would equal a third of what would have been with U.S. participation. 


\subsubsection{Government purchases in the project credit market}

Reviewing the national GHG emission reduction plans of Parties to the Convention, it is evident that governmental bodies will form a major component of the total demand for Kyoto units within the first reporting period. There are a number of governments within the EU who in particular will be major purchasers overt the first commitment period, including Italy $\left(198 \mathrm{MTCO}_{2} \mathrm{e}\right)$, Netherlands $\left(100 \mathrm{MTCO}_{2} \mathrm{e}\right)$, and Spain $\left(100 \mathrm{MTCO}_{2} \mathrm{e}\right)$. The governments of the other two major EU emitters, Germany and the UK, have decided that the Kyoto mechanisms will only be allowed to be used at the company level, with no acquisitions by governments planned. In its entirety, national governments in the EU have allocated 2,730 $€$ for the purchase of $106.8 \mathrm{MTCO}_{2} \mathrm{e}$ per annum from the Kyoto mechanisms. The federal government of Canada plans on purchasing approximately 80 $\mathrm{MTCO}_{2}$ e to meet its commitments (Canada, 2006), although future commitments to the $\mathrm{KP}$ are uncertain given recent changes in government priorities. The Japan federal government has given no indication that it will purchase emission credits via the Kyoto mechanisms, and no evidence is seen when surveying recent purchasing activity.

Based on the review of trading activity linked to government bodies in the past number of years and announced and planned activity, the range of emission reduction requirements attributable to governmental bodies will likely fall between 120 and 150 MTCO2e per year over the first commitment period.

\subsubsection{The European Union Emissions Trading Scheme}

Large implications for the global carbon market are originating from the ETS. The fundamental objective of the ETS is to reduce the costs in the EU attributed to reaching the goals of the Kyoto Protocol: with the ETS reductions have been estimated at 3.56 to 
4.55 billion USD compared to the estimated 8.36 billion USD without (EU-Commission, 2004). Targeted is a range of industrial activities responsible for upwards of $46 \%$ of the EU's GHG emissions. Of most importance to the global carbon market is the 'Linking Directive' contained within the ETS where participants are permitted to exchange certified emission reduction credits (CERs) or emission reduction units (ERUs), but not assigned amount units (AAUs) or emission removal units (RMUs), for EU allowances. As the ETS materializes over the next few years, there will be increasing demand for emission reductions, thereby making CERs and ERUs less vulnerable to competition from AAUs and RMUs. However, no linking credits will be allowed before the second EU-ETS phase (2008-2012), although CDM credits maybe be accrued and stored prior to 2008 for use once the second phase has begun.

The most activity related to corporate use of the Kyoto mechanisms will likely come from companies operating in Germany and the UK since the national governments of these countries have indicated in their national communications that it is corporate entities in the private sector rather than public entities who can actively engage in purchasing emission reductions from the Kyoto mechanisms. There are also large CDM projects that have also been developed by Japanese based firms whose national government is not making purchases in the carbon market. By early 2006, Japanese firms were involved in 18 projects contributing to over $32 \mathrm{MTCO}_{2} \mathrm{e}$ of emission reductions per year, of which they were the sole participants in eight projects totaling over $\mathrm{MTCO}_{2} \mathrm{e}$ per annum. 


\subsubsection{Net demand implications}

To summarize, some of the significant factors contributing to demand of carbon units will be corporate activity related to the ETS and other linked trading schemes that may develop, and demand from government bodies. The minimum demand by industry in Europe is about $45 \mathrm{MtCO}_{2} \mathrm{e}$ per annum. Corporate entities in Japan, Canada and Australia will likely contribute similar levels, and purchases by governments that have been announced are estimated to be in the order of $100 \mathrm{MtCO}_{2} \mathrm{e}$ per annum. This would mean an annual demand for CERs and ERUs of at least $200 \mathrm{MtCO}_{2} \mathrm{e}$ per annum. However, the demand by government and industry is likely to be closer to $300 \mathrm{MtCO}_{2} \mathrm{e}$ per annum when considering the recent purchase announcements by governments in Europe and others that are likely to materialize, the increasing trading volumes related to the ETS and the interest being communicated by Russia to link to this trading scheme. So far, the majority of emission credits purchased has been associated with CDM projects rather than JI. It is assumed that this trend continues towards the first commitment period and that CERs dominate over ERUs in most national emission inventories.

\subsubsection{Russian hot air and emission stabilization at 1990 levels}

In ratifying the Kyoto Protocol, Russia committed to stabilizing emissions at 1990 levels $\left(3,039 \mathrm{MTCO}_{2} \mathrm{e}\right)$ between 2008 and 2012. However, there is a wide range of projections for Russian emissions to 2010, and hence the amount of AAUs which may be available to the carbon market. The International Energy Agency (IEA) estimate $\mathrm{CO}_{2}$ emissions will be $854 \mathrm{MTCO}_{2}$ e below 1990 levels (72\% of the base year), while the Energy Information Agency suggest emissions will be $797 \mathrm{MTCO}_{2}$ e below 1990 levels (74\% of the base year). Meanwhile, Russia's third national communications (Nazarov et al., 2002) 
suggests emissions 340 and $760 \mathrm{MTCO}_{2} \mathrm{e}$ below the base year (75 and $88 \%$ of the base year), with the medium trajectory of $590 \mathrm{MTCO}_{2} \mathrm{e}(80 \%$ of the base year). Two main factors drive the range seen in these emission projections: differences in economic growth rates and differences in the energy intensity of the Russian economy.

After the collapse of the Soviet Union in 1992, Russia's GDP fell rapidly, decreasing a total of $42.5 \%$ in the six years that followed (Nazarov et al., 2002). This economic contraction lasted until approximately late 1998, after which time Russia's economy rebounded to grow by $6.4 \%$ in 1999 and $10.0,5.0,4.3$, and $7.3 \%$ in the years thereafter (METI, 2004). Russia's government hopes to maintain similar economic growth rates into the future, thereby doubling 2000 GDP by 2010 . Such objectives are reflected by the 'moderate' economic growth scenario described in the recently revised 'Energy Strategy 2020 for Russia' (EnergyStrategy, 2003), a document used by the Russian Federal government for energy planning and which therefore forms the basis for the country's third national communications. On a percentage basis, this moderate scenario equates to an annual average annual economic growth rate of 5 to $5.2 \%$ over this time. Alternatively, the IEA assume a much lower per annum growth rate between 2002 and 2010 of $4.4 \%$, reflected by the lower emissions projected. The economic growth rates assumed by the EIA are higher than the IEA as they take into account the higher economic growth that occurred in Russia between 2002 and 2004, but still fall below the ambitious projections within the Energy Strategy.

The projections of increased economic growth would propel emissions to the high end of the levels given above if concurrent efforts are not made to improve efficiency. This stems from the fact that Russia's economy is very energy intensive per unit of GDP, 
an evolution that has occurred in the years immediately after the Soviet state collapsed. During this time, GDP fell much faster than did aggregate energy use, and consequently the energy intensity of the Russian economy increased. The increase was rapid at the initial stages of the economic upheaval, equaling $2.6 \%$ in 1990 and $8.6 \%$ in 1991. Although there have been improvements in energy efficiency in most recent years, many of Russia's industrial sectors, including the electrical sector, still use two to three times the energy than compared to developed nations such as the U.S (IEA, 2002b; Grubb et al., 2004). In view of the scope for energy efficiency improvements in the economy as a whole, Russia's Energy Strategy (2003) states that energy demand could be halved by the year 2020 when compared with a baseline scenario of no measures being undertaken. Such energy efficiency improvements would have significant implications on carbon emissions into the future, especially for a country that relies on coal for a high proportion of its primary energy resources.

Similar to assumptions underlying economic growth, there are differences in assumptions on the energy intensity of Russia's economy within the various projections considered. The Russian Energy Strategy to 2020 suggests that energy demand could be cut in half by 2020 , inferring decreases in energy intensity of nearly $2.5 \%$ per annum. Similar to GDP, these rates are higher than those assumed in the two modelling efforts considered. The EIA assume that the energy intensity of EIT in general will decrease approximately $45 \%$ between 2002 and 2025 . Alternatively, the IEA assumes slightly lower decreases in Russia's energy intensity, with this falling less than $40 \%$ over a longer time period (2002 to 2030). Thus, these two sources assume annual increases in efficiency ranging from 1.45 to $1.95 \%$ per year. 
These two areas of discussion point to the major areas of uncertainty in Russia's potential surplus of AAUs. If the economy were to take a higher trajectory economic growth path, or if energy efficiency improvements did not occur as possible, then Russia's emissions would be higher and subsequently would limit the amount of Russian hot air available.

Uncertainties over Russia's available supply of AAUs also arise due to domestic pressures to limit emission sales in the first commitment period, including that:

- By restricting the sale of AAUs in the current commitment period Russia might benefit from higher prices in later crediting periods when targets may be set lower and the marginal cost for emission reductions higher (Bernard et al., 2003), and;

- Russia may be able to link domestic trading schemes with the ETS in the EU in order to attract investments in JI projects. However, if it opens up its AAUs for sale in the current commitment period this will likely reduce demand for JI projects and much needed, and also economically more valuable, infrastructure and energy technology projects related to JI (Moe et al., 2000).

These compounding factors likely mean that a much lower amount of Russia's available AAUs will be used in the 2008 to 2012 commitment period, likely ranging from 30-50\% of the available total. Based on the projections in the third national communications, this would equal between 185-300 $\mathrm{MTCO}_{2} \mathrm{e}$ per annum. 


\subsection{China in the global carbon market}

China is by far the largest developing nation when measured by population, economy, energy demand, and by GHG emissions. When measured by these parameters, it is also the most prominent nation that is not yet included within the UNFCCC framework in terms of having an emission reduction commitment. Per capita GHG emissions are presently only a $10^{\text {th }}$ of the United States or Canada but emissions are expected to rise significantly as China's economy continues to grow. A primary reason is that China's energy sector is dominated by coal. This source contributes over 70 percent of the energy in China and since China holds one of the largest reserves of coal in the world, coal will likely continue to dominate into the future. In this context, it is important to consider China's political and economic transition in regards to both its current and future status as a major GHG emitter, particularly in regards to the progression and development of its energy sector since it is this sector that is most important in regards to GHG emissions (fuel combustion contributes to over $85 \%$ of China's GHG emissions).

Over the past twenty years China's economy has grown at an annual average rate of 7\%, exceptionally fast relative to other developed or developing countries, while the country has moved from having a closed economy with strong government controls to one strongly influenced by market forces (Ogilvy, 2000). Alongside its strong economic growth, energy demand and consumption has accelerated throughout China, and particularly in the country's rapidly growing eastern provinces (Bao et al., 2002; $\mathrm{Ng}$ et al., 2006). During the initial years of its industrialization, China's industrial sector grew the quickest, and hence this sector of the economy has historically dominated total energy demand. However, China's population is now growing wealthier, and consequently 
energy demand in the residential, transportation, and commercial sectors is accelerating (Sinton et al., 2005). In terms of its energy use, relative to more developed nations, energy end-use efficiency is low in China across all sectors of its economy (Crompton et al., 2005). This is partially a consequence of the inability to finance more costly and technologically advanced equipment and processes and due to slower capital turnover in China historically.

The provision and supply of coal and other fuel sources in China is at different stages of deregulation and control by the state, and each have different economics and have developed differently in the context of China's economic and political transition (Wu, 2003). Broadly speaking, China is well-endowed with coal, but not gas and not oil. The state has sought to deregulate the coal sector, largely to encourage efficiency in the sector and to encourage foreign direct investment here. The economics of China's oil and gas sector influences the need for the state to have stronger control over the production and supply industries. Specifically, China has established onshore and offshore oil and gas production companies that are essentially monopolies in these different sectors. In the coal sector China maintains a relative trade balance with other nations - in some years exporting coal, and in some years importing. However, due to the scarcity of oil and gas in the country, it is increasing its imports and overseas acquisitions of these fuels.

\subsubsection{China position in future climate change mitigation regimes}

The International Energy Agency indicate that in 2002 China contributed 3,307 million $\mathrm{MTCO}_{2}$ to the atmosphere from energy consumption (IEA, 2005). This ranks China as the 2nd largest emitter globally. However, with the rapid growth of its export-driven economy, a reliance on coal as a fuel, and less efficient technology and energy 
conversion processes, China's carbon emissions are rising rapidly and by 2030 the country's contribution to global $\mathrm{CO}_{2}$ emissions is forecasted to surpass the U.S. (IEA, 2005). These are reasons why China is being heavily encouraged to partake in the Kyoto Protocol and in any related emission reduction framework that may materialize in the post-2012 period (Viguier, 2004).

China's active participation in the current global carbon market as a non-Annex I member is of significant economic importance to Annex I nations and the global economy as it has the most abundant low cost emission reduction possibilities of any region, and therefore supply of potential CDM projects. Estimates are that China has $35 \%$ to $45 \%$ of the estimated total CDM potential in 2010 , comparable to the estimated potential of Latin America, Africa and the Middle East combined (Jotzo et al., 2002; WB, 2004; Zhang et al., 2005). The World Bank (2004) suggests that there are just over 400 $\mathrm{MTCO}_{2}$ of potential abatement opportunities in China at a cost of 6 USD per tonne $\mathrm{CO}_{2}$, comparable to the price offered by the CERUPT programme in the Netherlands. This would equate to $80 \mathrm{MTCO}_{2} \mathrm{e}$ (where e = equivalent) of CERs per year over the 2008 to 2012 commitment period, enough to meet half of the demand for CERs if these units were to meet the majority of emission reduction requirements in the project credit market as suggested earlier. The primary reasons contributing to China's CDM potential are that, even after considering a $60 \%$ increase in energy efficiency since 1980 , China's industrial base remains inefficient, dominated by large, industrial units often reliant upon outdated technology and often powered by coal. In the electrical sector (which contributes up to $40 \%$ of China's GHG emissions), China requires $25 \%$ more primary resources than does Japan for each kilowatt hour of electricity generated (METI, 2004). 
Even though China has a vast potential for CDM projects, in the years between 2000 (when CDM projects could first be registered under the rules of the UNFCCC) and 2005 the majority of CDM projects were being implemented in Latin American countries, with virtually none in China. The reasons for this were largely institutional as China was slow to establish the necessary institutional and regulatory frameworks needed to facilitate CDM projects compared to its Latin American counterparts (Zhang et al., 2005). However, in 2005 China appointed the National Development and Reform Commission as the designated national authority to the UNFCC, and subsequently there has been a rush of CDM projects announced in China.

\subsubsection{China's CDM potential and the importance of the electrical sector}

Marginal abatement cost curves (MAC) are a common technique used to assess emission reduction possibilities both within the global economy and domestically within specific countries (Ellerman et al., 2001; Jotzo et al., 2002). Since MAC's measure the average cost for each additional unit of emissions reduced and likewise, these can be used to assess CDM potentials by country and by economic region. The World Bank (2004) derived MACs for China in order to study the potential for the CDM across different economic sectors, with the results indicating that the potential size of CDM activity in China might be equivalent to $79 \mathrm{MTCO}_{2}$ per annum by 2010 and that the most plentiful and economic GHG emission reduction opportunities were in the electricity sector (see table 2): 
Table 2. Emission reduction potential from CDMs by economic sector in China (from the World Bank, 2004)

\begin{tabular}{|c|c|c|}
\hline & & $\begin{array}{l}\text { Emission } \\
\text { reductions } \\
\text { per annum } \\
\text { (MTCO2) }\end{array}$ \\
\hline Sector & $\begin{array}{c}\text { Share } \\
(\%)\end{array}$ & $\begin{array}{c}\text { (2008 - } 2012 \\
\text { average) }\end{array}$ \\
\hline Steel making & 10 & 7.91 \\
\hline Cement & 10 & 7.91 \\
\hline Chemical industry & 5 & 3.95 \\
\hline Power generation & 50 & 39.53 \\
\hline Other sector & 15 & 11.86 \\
\hline Non-CO2 projects & 10 & 7.91 \\
\hline Total & 100 & 79.06 \\
\hline
\end{tabular}

The primary reason why China's electrical sector contains such significant opportunities for CDM projects is due to the heavy reliance on coal in the sector, and that the efficiency of generation technology is much lower than in more developed economies (Wang, 2003; Kroeze et al., 2004; Steenhof, 2006a).

\subsection{Chapter conclusions}

Factors affecting the global climate market have been reviewed, with China and its electricity placed in the context of this. First, the demand side of the market was studied by analyzing and summarizing the outputs from the third national communications of Parties to the UNFCCC showing that the Parties to the UNFCCC in their aggregate will be $566 \mathrm{MTCO}_{2} \mathrm{e}$ above their aggregate AAUs under current policies and measures. This reduces to $20 \mathrm{MTCO}_{2} \mathrm{e}$ below the Protocol targets if more rigorous mitigative efforts are undertaken before 2008. However, in both projections this amount is surpassed by the hot air available from Russia and the Ukraine. Based upon these two countrys' national communications, this will equal approximately $740 \mathrm{MTCO}_{2} \mathrm{e}$ in 2010 and upwards of 
$1,300 \mathrm{MTCO}_{2} \mathrm{e}$ if either economic growth slows down or efficiency increases at a faster rate than projected.

Although the above assessment suggests that the global carbon market will be in carbon deficit in 2010 relative to the objective of the Kyoto Protocol after considering Russia's surplus AAUs, there is significant demand for the purchase of ERUs from JI projects and CERs from CDM projects. Industrial players in Europe, who will likely dominate the private sector component of the carbon market in the first commitment period, are regulated from using AAUs from EIT to meet their emission reductions. On the supply side of the market, attention has been given to a number of major factors that will play a significant role in determining the available supply of Kyoto units. First are the surplus AAUs available from Russia and Ukraine. For this, the largest uncertainty comes from different possible trajectories of economic growth and change in Russia's economy. Finally, attention has been given to the role of China in the entire Kyoto process, the critical role and importance of it being included in the Kyoto market, and the dominating role of the electrical sector within this.

Summarizing the emission projections found in the national communications is advantageous since this can help illustrate how the carbon market may develop in the next few years, including the subsequent supply and demand of carbon units. The chapter has been concluded by drawing attention to the importance of China, and particularly China's electrical sector, in the global carbon market. With China's rapid economic ascension, the ongoing electrification of its economy, the reliance on coal for electricity generation, and the inefficiency of electricity generation technology in China make this sector ripe for low cost emission reductions, such as what could take place 
under the CDM found within the Kyoto Protocol. The sector offers a significant source for global mitigation of the GHG emissions that lead to anthropogenic climate change and therefore deserves the attention as given in this dissertation. 


\section{Methods to establish a GHG baseline in the electrical sector}

Synopsis: In this chapter, fundamental attributes of the methodologies underlying baseline construction and scenario development are reviewed, including how additionality is first established, the baseline methods selected for analysis, and the criteria that can be used to help determine the applicability of a baseline method.

\subsection{Chapter introduction}

The methodological fundamentals of the dissertation are reviewed. This review starts with the determination and assessment of additionality, a critical component for the successful operation of the CDM, followed by how baselines are created and their role within the determination of environmental additionality. Three different methods for baseline construction are considered, each based upon a fundamentally different perspective of the electrical sector - using a historical, current and forward looking perspective. Following this discussion, a review is given of scenario generation. Scenario generation and baselines are inherently intertwined since of critical importance to establishing a baseline is the creation of the business as usual scenario. The chapter concludes by discussing key criteria that can be used to help determine the applicability of a baseline method.

\subsection{Determining additionality}

The determination of additionality is the first task for a project developer and requires outputs from the baseline analysis on the volume of CERs associated with the proposed 
CDM project, as well as construction of alternative scenarios that can be used to argue points of the additionality assessment. Additionality is defined by the UNFCCC in terms of whether or not anthropogenic emissions of greenhouse gases by sources are reduced below those that would have occurred in the absence of the registered CDM project activity. A project that does not reduce emissions beyond the BAU would not be considered additional.

Additionality is an important component of the CDM since projects that do not go beyond the 'business as usual' in terms of GHG mitigation would not lead to further reduction of GHGs than would be expected. The concept of additionality has received much attention in the literature in terms of the idea that some of the most 'cost-effective' GHG mitigation projects (whether CDM, JI, or any other policy related initiative) may be the least 'additional' and that consequently strict project additionality would give perverse policy incentives in terms of these not being undertaken or promoted (Grubb, 1998; Sugiyama et al., 2001; Jotzo et al., 2002; Shrestha et al., 2002; Greiner et al., 2003). For example, demand side projects in the electrical sector may not be economically attractive when viewed purely from the benefits received from the potential sale of carbon credits related to the CDM or other GHG mitigation projections, even though they collectively present significant potential emission reductions (Shrestha et al., 2002). There have been a number of authors who have argued that the evaluation of potential GHG mitigation projects should be expanded to consider not only the GHG reduction benefits but also the "additional" economic benefits that would be derived. Overall, this would expand the pool of potential CDM projects (Deshun, 1999; Timilsina, 1999; Shrestha et al., 2002). 
Another concern is that countries could potentially limit domestic policies or measures that would reduce the additionality of endorsed GHG mitigation initiatives in order to increase the returns received (Shrestha et al., 2002). Here, the strategic delay of certain energy or technology policies or programs could potentially improve the chances for a CDM project to be considered environmentally additional. For example, China could withhold on establishing national level energy programs that would encourage the uptake of clean energy technology in order for these to have a greater chance of receiving credits for emission reductions. For China at least, recent events reduce the validity of these concerns. For example, the Renewable Energy Law was recently passed in order to promote the implementation of renewables into China's economy (PRC, 2006), while in the most recent 5-year plan, China's State Council announced a goal to reduce the country's energy intensity substantially over the next twenty years.

In terms of assessing additionality, the CDM EB provide a five-step 'additionality tool' (see figure 2) to help project developers identify if a project is additional. The tool was formulated through a process of public consultation by various academic, industry, and policy stakeholders (please see, for example, communications by CCMG, 2004; Esparta, 2004; Harthan, 2004; Kibona, 2004). It is based upon a number of widely used environmental and financial assessment techniques. The tool is used in this dissertation in a conceptual context to help identify hypothetical CDM projects that could be implemented into China's electrical sector. The details of this assessment are contained in Annex A. 
Figure 2. CDM Additionality Tool (from Ringius, 2004)

Now chart: Additionality scheme

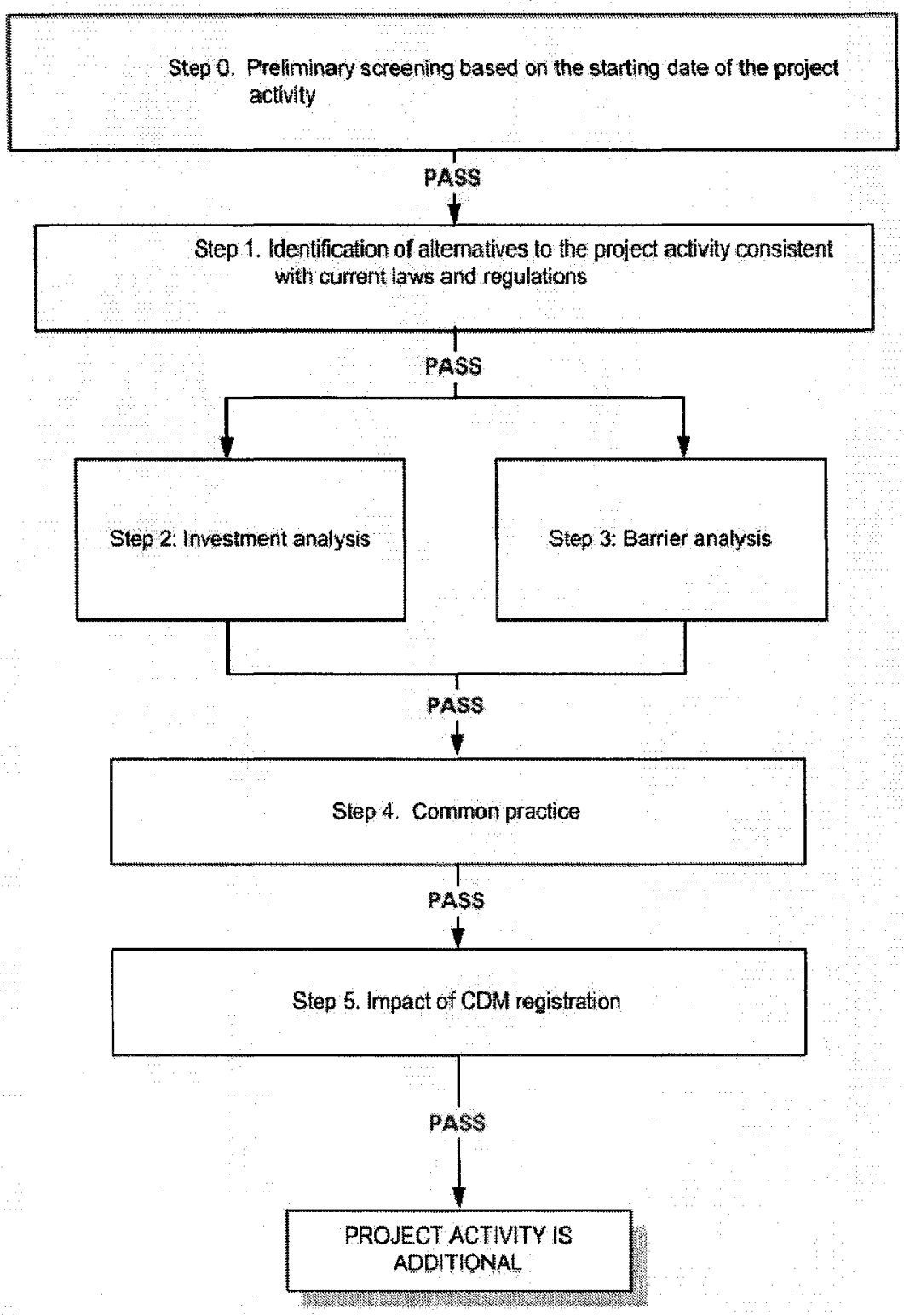

It starts with a preliminary screening based upon the starting date of the project and the provision of evidence that incentives from the CDM were seriously considered in the decision to proceed with the CDM project. The next step, Step 1, involves identification of the different alternatives for the project. If it is determined that the project is the only 
alternative (i.e. there are no other options), then the project is deemed non-additional because the CDM project would be the business as usual.

Step 2 and Step 3 can be undertaken in a complementary manner and involve both investment and barrier analysis. Investment analysis involves a quantitative analysis of a project to determine its economic viability with or without the revenue from emission credits. There are a number of analytical methods available for this, including simple cost analysis, investment comparison analysis, and benchmark analysis. The first of these is used only if the project will generate no economic or financial benefits other than the sale of carbon credits. The second are comparative based methods where carbon sales directly contribute to the financial viability (i.e. revenue - costs $=$ profit, where revenue includes addition of CERs) of an operational entity and typically draw upon accounting techniques such as the calculation of internal rates of return (IRR), net present values of carbon sales (NPV), or 'levelized' unit costs (which includes all costs, revenues and incentives/subsidies). The final option, benchmark analysis is based upon using a financial indicator and comparing this with a benchmark indicator that reflects standard returns in the market. If investment analysis does not clearly identify that a project will be the most financially attractive with the addition of CER revenue, barrier analysis can be undertaken. The essence of barrier analysis is to outline the various barriers that exist for the project, including those of a financial, institutional, technological, and economic nature.

The fourth component of the additionality tool involves determining if the project activity is 'common practice', namely if it likely will be implemented into the future under national energy plans. This therefore requires a thorough understanding of the 
policy landscape relevant to the potential CDM project. Step 5 summarizes if the CDM project is viable, and why project registration is a necessary element for the project to occur. Successful completion of the above confirms that the project is indeed additional and therefore project developers can continue with project design and implementation.

\subsection{Baseline standardization versus project specific baseline analysis}

Electrical sector baselines can be calculated at the individual project level or using a 'standardized' technique so that multiple projects can use the same baseline. There are a number of advantages and disadvantages of each approach. Project specific analysis can provide an accurate baseline for individual projects but it is more labour intensive, with the resulting additional cost limiting or even erasing the added benefit of emission reductions (Sathaye et al., 2004). Since each project is unique, including the data that is required to produce a baseline, this methodology is not readily reproducible or transferable. This limits its use to projects which exceed a threshold for total CERs produced, thereby limiting the number of smaller CDM projects which might exist otherwise. Another issue is the inapplicability of this approach to potential CDM projects in the demand sector. Here, project specific analyses exclude important system variables and dynamics that determine how the emission intensity of electricity available to the end-user might change over time. Numerous authors and studies have therefore suggested that electrical sector baseline methodologies should be at a multi-project level (Bosi et al., 2002; Kartha et al., 2004). Kartha et al. (2004) document a range of initiatives that have attempted to standardize emission baselines, including initiatives by 
the OECD/IEA, Japanese Government, U.S. Voluntary Reporting Program and Canada's Greenhouse Gas Emissions Reduction Trading Program (GERT).

Since multi-project CDM baseline (MPB) methodologies calculate emission reductions based on electricity made available over a grid, individual projects do not operate in isolation but are part of a larger electrical system made up of many individual generators. This is a highly dynamic and complex system, with changes in technology, resource availability, and environmental constraints all playing a role in determining its attributes. Larger scale economic, political and institutional structures often play a major role in determining the evolution of each of these factors, thus making these an intrinsic part of the electrical system. This point is especially relevant in light of the liberalization processes sweeping many national electricity markets in the world.

Applying a standardized emission baseline in the electrical sector still presents a number of fundamental challenges, including that different types of electricity generation plants are operated as base-load load plants with high capacity factors, and some acting as peaking plants used only when there is high demand for electricity. If a CDM project developer were able to identify the type of generation plant being displaced with the construction of a CDM eligible project, then this would be straightforward. This is rarely known and as such a series of different techniques are available to the baseline practitioner.

\subsection{A review of multi project baseline methodologies}

Three techniques to generate a MPB suggested for use by the CDM EB include dispatch analysis, build margin and operating margin analysis, while a fourth technique investigated is decomposition analysis. The basic underpinnings of each approach, the 
project types that they are most applicable for, and the technical and data requirements for each are briefly reviewed in the sub sections that follow (see table 3 for a summary).

Table 3. Characteristics of different multi-project baseline methods

\begin{tabular}{|l|l|l|l|l|}
\hline & Dispatch analysis & Build margin & \multicolumn{1}{|c|}{$\begin{array}{c}\text { Operating } \\
\text { margin }\end{array}$} & $\begin{array}{l}\text { Decomposition } \\
\text { analysis }\end{array}$ \\
\hline $\begin{array}{l}\text { Project types } \\
\text { of most } \\
\text { relevance }\end{array}$ & All & $\begin{array}{l}\text { Base-load } \\
\text { (coal, large } \\
\text { capacity natural } \\
\text { gas) }\end{array}$ & $\begin{array}{l}\text { Peak-load } \\
\text { (turbine natural } \\
\text { gas, wind, } \\
\text { small hydro) }\end{array}$ & All \\
\hline $\begin{array}{l}\text { Data } \\
\text { requirements }\end{array}$ & $\begin{array}{l}\text { Detailed, bottom-up } \\
\text { boiler/generator level } \\
\text { database, fuel prices, } \\
\text { technology } \\
\text { characteristics and } \\
\text { price, electricity } \\
\text { demand data } \\
\text { (optimally by hour) }\end{array}$ & $\begin{array}{l}\text { Source specific } \\
\text { electricity } \\
\text { generation data } \\
\text { for two sets of } \\
\text { years }\end{array}$ & $\begin{array}{l}\text { Source specific } \\
\text { electricity } \\
\text { generation data } \\
\text { for one year }\end{array}$ & $\begin{array}{l}\text { Historical data } \\
\text { on electricity } \\
\text { demand and } \\
\text { supply }\end{array}$ \\
\hline Model & Dispatch model & Equation based & Equation based & Equation based \\
\hline
\end{tabular}

\subsubsection{Dispatch analysis}

Dispatch analysis is a method commonly used by utilities to plan for capacity additions in grid areas (EPA, 2004), but also has been a method that has been applied in numerous CDM baseline studies in the electrical sector (Lazarus et al., 2001). It is the method emphasized by the CDM EB when adequate information and technical requirements are available. This approach relies on a detailed, bottom-up boiler/generator level database containing cost and operational characteristics of every boiler and generator providing electricity to national or regional grids.

Dispatch analysis assumes that an electrical system will operate in an economically optimal way: when the objective is to minimize costs, the dispatch model will determine the most cost-efficient route of development when considering how future 
fuel, capital, labour and environmental costs might change. Here, the lowest running cost capacity (such as hydro, nuclear and base-load coal) will contribute to the majority of hours in the load curve, while capacity with high or variable operating costs (such as natural gas turbines) will only be used in peak hours. A peak-load CDM project in the electrical sector, such as a gas turbine natural gas plant, will decrease or affect required capacity at the top of the above curve, while a base-load plant would affect capacity at a point at the bottom of this curve.

When considering a dynamic and rapidly evolving system, dispatch analysis therefore provides a powerful tool that can be used to plan for capacity additions when considering the breadth of economic and technological factors contributing. Using this tool, multiple scenarios can be run based upon changes in key drivers of the electrical sector, such as changes in electricity demand, fuel availability and cost, technological details of generation units, environmental constraints, or transmission availability.

\subsubsection{Using dispatch analysis to generate electrical sector emissions}

The dispatch model uses linear programming to 'solve' a series of mathematical equations and parameters that represent the complexities of electricity generation, transmission and distribution. Linear programming assumes optimization amongst the various parameters interacting within the model and is based upon an 'objective function' that must be either minimized or maximized. For the purposes of CDM baseline analysis, this objective function states that the full life cycle costs of the generation capacity used must be minimized. This objective function can be written algebraically as follows (ICF, 2004a): 


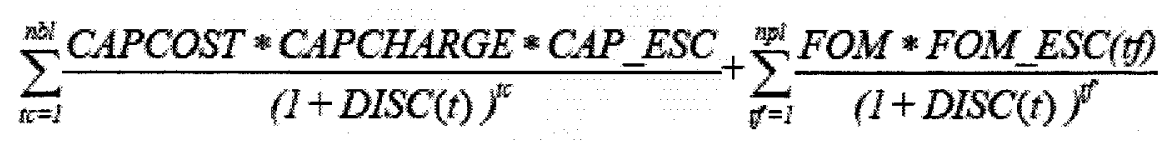

Where:

CAPCOST $=$ Capital cost of the plant (including interest payments incurred during construction);

CAPCHARGE $=$ Capital charge rate for the particular plant being modeled. This is used to annualize capital costs and is a function of the book life of the plant, discount rate, tax rates, capital structure, etc;

CAP_ESC $=$ Capital cost escalation factor for the run-year;

DISC $(\mathrm{t})=$ Discount rate for year $\mathrm{t}$;

FOM = Fixed operation and maintenance costs;

FOM_ESC $(\mathrm{tf})=$ Fixed operation and maintenance escalation factor for year $\mathrm{tf}$;

tc $=$ Indexes all calendar years in the book life of the plant up to the last year in the study period;

$\mathrm{nbl}=$ Total number of calendar years in the book life of the plant up to the last year in the study period;

$\mathrm{tf}=$ Indexes all calendar years in the physical life of the plant up to the last year in the study period;

$\mathrm{npl}=$ Total number of calendar years in the physical life of the plant up to the last year in the study period. 
In addition to the above, variables are also defined and included in the objective function to represent the different operation, maintenance and fuel costs attributed to the running of each generation unit on a temporal scale ranging from hours to years that in turn must also be minimized. These above costs are defined by the present sum of all the components across all the years considered. In algebraic terms this is written as:

$$
\sum_{j=1}^{n} \frac{V O M x V O M \_E S C(t)+F U E L(t)}{(1+D I S C)}
$$

Where:

$\mathrm{VOM}=$ Variable operation and maintenance costs;

VOM_ESC $(\mathrm{t})=$ Variable operation and maintenance cost escalation factor for year $\mathrm{t}$ FUEL $(t)=$ Fuel costs for year $t$;

$\mathrm{t}=$ Indexes all calendar years mapped to a given run-year, and;

$\mathrm{n}=$ Total number of years mapped to a given run-year.

\subsubsection{Technical and data requirements}

In order to establish a baseline using this method, a dispatch model of the specific electrical sector where the CDM project is being proposed must exist. The user must determine the time period of the baseline and the drivers or constraints to be manipulated. 
The China Integrated Planning Model (China IPM) (initially developed and operated by ICF International) was used in this analysis (ICF, 2004a). A series of drivers and constraints were considered, including:

\section{Drivers:}

- Electricity demand by province and year

- Peak-load requirements by province and year

- Fuel prices (coal, natural gas, diesel) by region and year

- Technology costs by year

- Technology efficiency by year

\section{Constraints:}

- Sulphur dioxide emission limits

- Renewable energy targets

- Constraints on the availability of fuels

- Established nuclear-powered electricity development program

To include constraints into the modelling framework, emission fees were added to the cost of electricity generated so as to account for emission targets, minimal renewable energy goals were included by setting regional minima for these, minimal regional nuclear capacities were established for scenarios where these efforts were successful, and regional maximums were put in place to reflect that only so much natural gas-fired electricity was able to be built. A parameter not manipulated were grid interconnections as this was ascertained by research completed by ICF International (ICF, 2004b; a). 
Sixteen different regions are included in IPM, with each assuming appropriate values for the range of drivers considered.

\subsubsection{Operating and build margin analysis}

If sufficient information is not available for dispatch modelling analysis, the CDM EB recommend the use of either operating margin (OM) or build margin (BM) analyses, or a combination of these two (a combined margin (CM)). These forms of baseline methodologies are explained and formulized below.

\subsubsection{The operating margin approach}

The operating margin approach is derived from the average emission intensity of the entire grid when considering all fossil-fuel generated electricity. Therefore, it considers the operational characteristics of every type of fossil-fuel based capacity within its analytical framework, regardless of the age of the underlying generation technology. Since it considers the average emission intensity of the entire grid, this approach is best suited for generation capacity that contributes to base-load power only on an intermittent basis, such as the case for wind, solar or small hydro CDM projects.

There a number of ways the emission intensity at the operating margin can be determined:

- By the load contribution of each type of plant (i.e. number of hours operated per year)

- By the capacity mix if load duration information is not available (i.e. by the contribution of each type of plant to total capacity of the grid) 
The second approach is used as information on load duration on a plant basis was not available. This is formulized as follows:

$$
O M_{\text {Coef }}=\left(\sum_{\text {FuelType }} G W h \times G W h_{E f f} \times F u e l_{\text {Coef }}\right) / G W h_{\text {Thermal }}
$$

Where:

$\mathrm{OM}_{\mathrm{Coef}}=$ the emissions coefficient constructed using the operating method;

$\mathrm{GWh}=$ the amount of electricity generated by fuel type;

$\mathrm{GWh}_{\mathrm{Eff}}=$ the efficiency of fossil-fueled electricity generation capacity;

Fuel $_{\text {Coef }}=$ the fuel specific emission factors, optimally specific to the region involved or the defaults provided by the IPCC;

$\mathrm{GWh}_{\text {Thermal }}=$ the amount of thermally generated electricity

\subsubsection{The build margin approach}

A different baseline approach is commonly employed for electrical sector projects that displace base-load power and is referred to by the CDM EB as the build margin (BM). The BM approach seeks to develop a proxy for what type of power plant would have been built to meet the electricity demand should a base-load related project not be implemented. This is done by deriving an emissions factor that reflects all recently constructed thermal plants that can be used to help indicate the characteristics of new capacity additions. For large-scale projects that will displace new base-load plants, this 
approach is therefore considered more accurate than the OM approach as it is based on plants recently built or currently under construction (typically within a three to five-year period). However, as noted by Sathaye (2004), some electricity grids (such as those in South Africa or regions in Russia) have not built new capacity in nearly 10 years, while other grids serving regions with high economic growth (such as in the eastern regions of China) have experienced high rates of capital construction in recent years. As such, the decision on the vintage of electricity capacity to include in the BM must be specific to the region under study. It is also dependent on the direction of policy concerning the electrical sector under question. For example, if the energy or environmental policies of a country stipulate changes in generation mix, technology or fuel mix, this will affect the rate of change in the $\mathrm{BM}$.

The basic approach used to calculate this emission factor is to determine the changes in capacity between two years by generation source, by fuel type and by technology. This information can then be used to calculate a weighted emission factor by multiplying the contribution of thermal power to total electricity generated against the aggregate operating margin emission factor (as calculated in the previous section). This can be formulized as follow:

$$
B M_{\text {Coef }}=\left(\left(\frac{G W h_{\text {Thermal }, T o}-G W h_{\text {Thermal }, T n}}{\sum\left(G W h_{\text {Source }, T o}-G W h_{\text {Source }, T n}\right)}\right) \times O M_{E f f}\right.
$$

Where: 
$G W h_{\text {Thermal } T_{o}}=$ electricity generated by thermal sources in most recent year

$G W h_{\text {Thermal, }, n}=$ electricity generated by thermal sources in previous year to current

$G W h_{\text {Source,To }}=$ electricity generated by all sources in most recent year

$G W h_{\text {Source.Tn }}=$ electricity generated by all sources in previous year to current

\subsubsection{Top down baseline methodologies: factor decomposition}

A top down approach to baseline construction represents a fundamentally different method than the bottom up approaches incorporated into the dispatch and BM analyzes, and a comparison of these in terms of accuracy and other indicators could be of value to the ongoing developments in baseline methodology as well as having relevance academically and to industry. Factor decomposition is a key example of such a top down approach that has been used to assess the energy and environmental attributes of energy systems (Ang et al., 1997; Ang et al., 1999; Lee et al., 2001; Paul et al., 2004), including examples of CDM baseline analysis (Nag et al., 2005; Steenhof, 2006a). It is a method where the historic factors that determine changes in energy or emissions in an economy or economic sector are isolated (such as increases in economic activity or changes in energy efficiency), and their influence as an explanatory variable assessed. This allows these factors to be used inside of equation based models to study the future development of a system though sensitivity testing. 


\subsubsection{Decomposition fundamentals}

The basic approach of decomposition analysis is to compare a factor's value in a base year to one or a series of later years. The first of these is referred to as a period-wise approach as it considers two years in the analysis; the base year and a later year, with the latter typically chosen at least 10 years out from the base year. The second approach applies decomposition equations across each subsequential pair of years over the period being considered. If an understanding of how factors that influence the term being decomposed change with time is desired, then the latter approach of decomposition is the preferred method. This technique can also allow a more visual or graphical representation to be given of analysis results, aiding in both the interpretation and presentation of results. Time series approaches either use a rolling base year or use an annually changing weighting scheme. The choice whether to use a periodwise or time series approach is one of the first major methodological choices of the analyst using decomposition analysis.

\subsubsection{Factors affecting electricity demand and emissions from electricity generation}

Energy sector decomposition analyses are typically constructed upon three underlying drivers; structural effects within the economy as a whole or of specific economic sectors, energy intensity effects in the economy as a whole or of individual economic sectors, and activity effects that measure the impacts of changes in economic activity and output. Studies that have extended the decomposition method to the electrical sector generally consider structural effects in terms of the generation mix of electricity (e.g. thermal, 
hydro, nuclear, renewables) and/or the contribution of fossil fuel shares (e.g. coal and coal products, petroleum products and natural gas). Intensity effects measure the energy efficiency of electricity generation, but may include other factors which reduce system efficiency, such as transmission and distribution (T\&D) losses and electricity demand by producers (Ang et al., 1998; Shrestha et al., 1998).

The factors that commonly affect electricity demand, the denominator in the electricity intensity coefficient, and secondly emissions, the numerator are summarized (see table 4): 
Table 4. Factors governing electricity demand and the emission characteristics of its generation

\begin{tabular}{|c|c|c|}
\hline Parameter & Definition & Effect \\
\hline \multicolumn{3}{|c|}{ Electricity demand } \\
\hline $\begin{array}{l}\text { Population } \\
\text { growth/per capita } \\
\text { income }\end{array}$ & $\begin{array}{l}\text { Changes in aggregate population } \\
\text { or per capita income }\end{array}$ & $\begin{array}{l}\text { Results in increased energy } \\
\text { consumption if energy use per capita } \\
\text { remains constant or rises }\end{array}$ \\
\hline $\begin{array}{l}\text { Changes in } \\
\text { economic } \\
\text { activity/industrial } \\
\text { output }\end{array}$ & $\begin{array}{l}\text { A measure of changes in } \\
\text { economic activity or industrial } \\
\text { output, typically measured in } \\
\text { dollar terms }\end{array}$ & $\begin{array}{l}\text { As economic activity increases, } \\
\text { without an increase in energy } \\
\text { efficiency, energy demand will rise } \\
\text { based on the energy intensity of } \\
\text { products being produced }\end{array}$ \\
\hline Structural change & $\begin{array}{l}\text { Proportional changes in one or a } \\
\text { series of economic sectors in } \\
\text { context to total output }\end{array}$ & $\begin{array}{l}\text { If proportional economic output } \\
\text { from sectors with low electricity } \\
\text { intensity were to rise, total } \\
\text { electricity intensity would decline in } \\
\text { absence of other changes }\end{array}$ \\
\hline Energy intensity & $\begin{array}{l}\text { The amount of energy used per } \\
\text { unit of economic activity }\end{array}$ & $\begin{array}{l}\text { If the energy intensity of a } \\
\text { sector/product changes, overall } \\
\text { energy demand will change }\end{array}$ \\
\hline \multicolumn{3}{|c|}{ Emission characteristics of electricity generation } \\
\hline Generation mix & $\begin{array}{l}\text { Mix of primary energy sources } \\
\text { used to generate electricity (e.g. } \\
\text { thermal, hydro, etc) }\end{array}$ & $\begin{array}{l}\text { Switches to lower or zero carbon } \\
\text { sources will decrease emissions and } \\
\text { vice versa }\end{array}$ \\
\hline Fossil fuel mix & $\begin{array}{l}\text { Mix of fossil fuels used to } \\
\text { generate thermal derived } \\
\text { electricity }\end{array}$ & $\begin{array}{l}\text { Switches to lower or zero carbon } \\
\text { fuels will decrease emissions and } \\
\text { vice versa }\end{array}$ \\
\hline $\begin{array}{l}\text { Energy } \\
\text { efficiency of } \\
\text { generation }\end{array}$ & $\begin{array}{l}\text { The technical efficiency of } \\
\text { conversion of primary resources } \\
\text { into electricity }\end{array}$ & $\begin{array}{l}\text { Increasing energy efficiency } \\
\text { decreases fuel requirements and } \\
\text { emissions and vice versa }\end{array}$ \\
\hline $\begin{array}{l}\text { Transmission } \\
\text { and distribution } \\
\text { (T\&D) losses }\end{array}$ & $\begin{array}{l}\text { The technical efficiency of the } \\
\text { T\&D network to deliver } \\
\text { electricity }\end{array}$ & $\begin{array}{l}\text { T\&D losses act to increase the } \\
\text { emission intensity of electricity } \\
\text { consumed by end-user }\end{array}$ \\
\hline $\begin{array}{l}\text { Auxiliary } \\
\text { consumption of } \\
\text { power }\end{array}$ & $\begin{array}{l}\text { Actual power consumed by an } \\
\text { electricity generation station in } \\
\text { its operation. }\end{array}$ & $\begin{array}{l}\text { Auxiliary consumption of power } \\
\text { acts to increase the emission } \\
\text { intensity of electricity consumed by } \\
\text { end-user as more electricity must be } \\
\text { generated }\end{array}$ \\
\hline
\end{tabular}

\subsubsection{Factor decomposition analytical approaches}

There are two general approaches to decomposition commonly used: Index

Decomposition Analysis (IDA) and Structural Decomposition Analysis (SDA). These 
two approaches are different in the context of their analytical structure and the applications each are best suited to as SDA approaches draw upon input-output (I-O) tables and matrix algebra, whereas IDA uses indices. Therefore SDA is often used in economic analyses concerned with the flows of commodities within an economy, such as to understand how flows of goods and services might be impacted by policy, trade tariffs, economic incentives, etc. Indices, alternatively, are used to give a higher order representation of complex data, and include examples such as financial indices used to measure the performance of a stock market or consumer price indices (CPI).

There are a number of weaknesses and strengths of either approach. Due to the data it uses and the incorporation of input-output coefficients, SDA is able to include indirect effects of changes in the structure of output. This is because the Leontief inverse used in the I-O model captures indirect effects, allowing analysts to understand how changes in one parameter might change demand for another sector or parameter. There are a number of limitations of using the SDA method, however. Input-output tables must exist for the region and industrial sectors considered, but often these I-O tables are only relevant for a short period of time in terms of economies experiencing rapid economic change (such as in China).

Alternatively, IDA is more accessible as it can be used with data that is more readily available while outputs are easier to understand by analysts and end users. It also has the ability to consider important details because the information it draws upon is "bottom up" in nature, including technical data on energy technologies and the efficiency of these technologies. This approach gives users the ability to consider a broader range of indicators of changes within an energy system, such as technical details of fuel mix, 
energy efficiency, transmission infrastructure or auxiliary use of power. Another advantage of the IDA approach is that it is able to include a temporal dimension more readily than SDA approaches. Considering these factors, the IDA approach is selected as the decomposition approach used in the decomposition analysis completed in this research.

\subsubsection{Index decomposition analysis techniques}

Within IDA, there are a number of general techniques used, but with no preference evident in the literature as the exact approach used for decomposition analysis is often reliant on the problem studied (Ang, 2004). In the past decade the Laspeyres and the Divisia index methods have been widely used, while most recently logarithmic based approaches built upon the Divisia index method approach have been proposed based on the ability of these to produce a 'perfect decomposition' (i.e. a low or non-existent residual term) (Ang, 2004).

Both Divisia and Laspeyres indices are mathematical representations of the component parts of a trend over time, which each working to decompose the following basic identity when the interest is the total change in energy consumption or emissions: 


$$
E=e A
$$

Where:

$\mathrm{E}=$ total energy use/emissions associated with activities being analyzed, such industrial output in the industrial sector, electricity demand in the electrical sector, kilometres traveled in the transportation sector, etc;

A = total output or activity, and;

$\mathrm{e}=$ aggregate energy/emission intensity of activities analyzed (in terms of energy use/emissions per unit of output).

Alternatively, an analyst may be interested in understanding how the energy or emission intensity of a system has changed with time. This is represented by the following identity:

$$
E I=E / A
$$

Where:

$\mathrm{EI}=$ aggregate energy/emission intensity, and;

$\mathrm{E}=$ total energy consumption/emissions, and $\mathrm{A}=$ activity (e.g. KWh of electricity demanded). 
There are a number of basic characteristics of each approach. First, the Divisia index operates as a weighted sum of logarithmic growth rates of the factors being studied (such as aggregate emissions or emissions intensity), where the weights are each components' (e.g. economic sector) share of the total value, with this given in the form of a line integral. The Laspeyres form of decomposition, alternatively, is largely based upon ratios and how factors change with time. The building block of methods linked to the Laspeyres index therefore are tied into the concept of percentage change, whereas the building block of methods linked to the Divisia index is based on the concept of log (i.e. logarithmic) change (Ang, 2004). As suggested, there appears to be no preferred method considering these two alternative forms when surveying their use in the literature.

Compared with the Laspeyres index, the Divisia index has a number of advantages. First, the decomposition formulae can be easily extended to include more than two factors without greatly increasing mathematical complexity. Secondly, because of how its weighting scheme is structured the Divisia index is more closely associated with changes in time, hence the unaccounted residuals are relatively small. However, this points also to a disadvantage. Since weighting is done arbitrarily based upon the proportion of energy/emissions of a particular sector to the aggregate, this therefore assigns interaction terms to the factor being indexed. The implication of this is that results can sometimes be difficult to interpret. A related limitation of the logarithmic weight function is its inherent assumption of a constant growth rate. This is especially relevant to the Chinese electrical sector seeing both its rapid growth over the last 20 years 
and that with higher growth rates, even small changes between years can result in significant differences in energy consumed or emissions released.

By comparison, the Laspeyres index approach has a number of advantages: the decomposition components can be easily understood and determined, and that the interaction terms, hidden in the Divisia indices, are explicitly accounted for. A major disadvantage of the Laspeyres method, however, is that as the number of factors increases, the interaction terms soon become complicated and difficult to manipulate and interpret. A third disadvantage occurs when the Laspeyres index approach has weights that are tied with the values of the initial year. Here, when changes in the factors between the initial year and the terminal year are non-marginal, the interaction terms can be substantial.

These two approaches can be further categorized into multiplicative and additive techniques, with these incorporating three different types of indicators: absolute, intensity and elasticity. The additive decomposition approach is used to determine factors leading to changes in absolute energy/emissions, while the multiplicative approach can be used to determine intensity and elasticities of energy/emissions. The use of one or combination of each depends upon the objective of the analysis being undertaken. In multiplicative decomposition the "ratio" change of an aggregate is decomposed, whereas in the additive case its "difference" change is decomposed (Ang, 2004). The multiplicative form therefore describes the relative growth of an indicator into its determinant effects across a time series $(t 1, t 2, \ldots t n)$, whilst the additive form describes the absolute difference. Nonexperts in the field of decomposition are more apt to understand additive forms of 
decomposition, although multiplicative approaches are easiest to understand in terms of the use of normalization and the ease of graphical display.

\subsection{Applying scenario analysis}

Scenarios form an important component of baseline analyses as these help practitioners to ascertain the projection of future emissions likely to occur when considering the economic, financial, technological, regulatory and political circumstances within which the emission reduction activity exists (Alcamo et al., 1996b). In terms of this research, a range of possible futures are envisioned and applied for several reasons. First, this allows the baseline methods to be tested in terms of how they are able to embrace the main uncertainties underlying emission projections. Secondly, using different scenarios will help to test the additionality of the CDM project case studies in different economic circumstances, a suggestion given in the additionality tool created for the purposes of the CDM. For example, in a world where natural gas-fired electricity becomes more cost competitive with coal-fired electricity, the additionality of natural gas based CDM projects will be reduced.

\subsubsection{Scenario fundamentals}

(Emission scenarios are) projections of the future state of the society and environment based on specific assumptions about key determinants such as population, economic growth, technological change, or environmental policies (Alcamo, 2001. pp. 7)

The above definition emphasizes that scenarios are built upon assumptions of key

'drivers' of the end points (e.g. future state of society or environment) being examined.

These drivers are often decision points that corporate leaders, national governments, or 
policy makers target in order to stimulate a desired change or, in some cases, constitute key factors that influence decision and policy-making. The information provided in such a scenario exercise can offer a picture of the effects of decision-making relative to the end point being considered. In this context, scenarios are ideal tools because they can be used to develop storylines relevant to progression of key drivers in the electrical sector that impact on greenhouse gas levels.

Given their usefulness for informing and contributing to decision-making, scenarios have been widely used in other analyses for purposes ranging from the development of business strategy, energy demand projections, and national and international energy and environmental policy formulation. A prominent example of scenario analysis specific to energy-related greenhouse gas emissions is the Special Report on Emissions Scenarios (SRES) by the Intergovernmental Panel on Climate Change (IPCC) (IPCC, 2000a). This report surveyed over 160 energy and emissions scenarios in the literature, culminating in a scenario exercise where four families of scenarios were envisioned, each consisting of its own subset of emission trajectories that incorporated differences in underlying drivers of emissions. Similarly, the International Institute for Applied Systems Analysis (IIASA -WEC) derived and assessed a series of global energy scenarios to the year 2100 to gain insight on the environmental, economic, and security impacts of these (Alcamo et al., 1996a; Nakicenovic et al., 1998; Parikh, 1998; Nakicenovic, 2000). 


\subsubsection{Generally accepted practices for scenario development}

There are a number of general practices for scenario development that have been established in the scenarios literature (Alcamo et al., 1996a; Nakicenovic, 2000). These include:

- Ensuring that there is internal consistency within scenarios envisioned, and;

- That all assumptions used are realistic and plausible.

The importance of these parameters is explained further by Grübler and Nakicenovic (2001 pp. 412), two lead authors of the SRES, who write:

...the future is unknown, and each future is 'path-dependent': that is, it results from a large series of conditionalities ('what if... then' assumptions) that need to be followed through in constructing internally consistent scenarios. Socio-economic variables and their alternative future development paths cannot be combined at will and are not freely interchangeable because of their interdependencies. One should not, for example, create a scenario combining low fertility with high infant mortality, or zero economic growth with rapid technological change and productivity growth - since these do not tend to go together in real life any more than they do in demographic or economic theory.

There is a range of methods available for baseline practitioners to assess the degree to which a driver is unknown, and to communicate this to policy makers, companies, or other stakeholders (EEA, 2005). In order to address uncertainty, the UNFCCC suggests using conservative assumptions in any scenario formed. By using conservative assumptions (e.g. that more versus less coal-fired capacity will be developed relative to cleaner burning natural gas plants, or that there are not large increases in the efficiency of 
generation that exceed historical occurrences), baseline practitioners will also ensure that the high range of possible emission intensities is used in the electrical sector occurs.

\subsubsection{Approaches for scenario analysis}

Approaches to scenario formulation are strongly influenced by the perspective of the scenario practitioner, which in turn influences the techniques eventually used for scenario formation. Some scenarios follow a forward-looking approach, relying on past events to give insight into the future, while others follow a backward-looking or normative approach, where practitioners pick a point in the future and work backwards (Dreborg, 1996; Hunhammar, 2001; Robinson, 2003). A forward-looking scenario, therefore, is rooted in the belief that the future can be best understood by using explanatory approaches derived from statistically derived historical relationships, while normative scenarios follow the rationale that the path to the future can be best understood only if the end point is already established. Since normative scenarios are not derived from past events, these generally utilize qualitative techniques, while forward-looking scenarios generally rely upon quantitative techniques that draw upon statistical relationships, correlations, and empirically derived formulations.

\section{Forward-looking scenarios}

Forward-looking scenarios take past occurrences and use these as a basis to explore plausible future developments. Central to this is formulating a set of assumptions on policies, measures and key driving forces (EEA, 2004). For this reason, these scenarios are evolutionary in development and do not take any one future as being either desirable or undesirable (Nakicenovic, 2000). The plausibility of any one scenario is usually tested 
by a thorough review of related scenarios and literature, of comparison of different modelling techniques, and through processes of peer review. The process allows creation of multiple narrative storylines that contain differences in driving parameters and outputs that also can be considered plausible and realistic.

A prominent example of a scenario development exercise using such an approach was the generation of scenarios included it the Special Report on Emission Scenarios (SRES) by the IPCC (IPCC, 2000a). The SRES scenarios were developed by a group of academic, policy, and industry stakeholders involved with aspects of global change research and global emission scenario analysis, where the first building block was to complete a survey of the literature and scenarios related to global emission projections. This process allowed the range of driving factors underling each scenario (including population growth, economic development, energy consumption, rates of technological change, and land-use change) and the interconnections between these to be ascertained. 'Narrative' story lines were then developed based upon this review, with these in turn used to help guide the quantitative analysis undertaken. In order to ensure consistency of modeled outputs, five different models were used to undertake this analysis. The final step undertaken by the SRES team was the selection of one particular scenario as the 'marker' scenario for each scenario family deemed best able to represent the fullest range of driving forces within each scenario family.

\section{Normative approaches}

Normative approaches, also referred to as backcasting or anticipatory approaches (EEA, 2005), take a picture of the future and map out different possible paths to this future. 
Unlike forward-looking scenarios where there are inexplicit values contained within assumptions used, this is an explicitly value laden approach for scenario analysis.

Backcasting has roots that run counter to the deterministic and reductionistic approaches that forward-looking scenarios traditionally rely upon. Criticisms of this latter approach have often highlighted the risks of trying to reduce an uncertain future into deterministic equations or even probabilistic theory (Demeritt, 2001), with these in turn used as arguments to promote the concept and method of backcasting (Lovins et al., 2001; Robinson, 2003). Backcasting differs from deterministic approaches as it does not extrapolate current trends and developments but takes a possible future image as a starting point and then reasons backwards to the present situation. Although it is not deterministic in nature, it often does contain individual predictive calculations (e.g. the likely effect of a change in population growth rates or in technological change) (Robinson, 2003).

\section{Contrasting explanatory and normative approaches}

In the context of CDM baseline methodologies, explanatory and narrative approaches for scenario development are much more suited than normative approaches. The primary reason for this is that normative approaches are inherently value laden, based on utopias or envisioned policy successes. CDM baselines, alternatively, are concerned not with a picture of how the world could evolve under optimal or other value laden conditions, but are based on the most conservative projection of the future as possible considering policies and processes that will most likely occur. Therefore, an explanatory scenario approach is used in this research. 


\section{Qualitative and quantitative techniques}

Normative and explanatory approaches are suited for either qualitative or quantitative techniques for actual scenario formulation. Normative approaches for scenario development are qualitative by their inherent characteristics as they are concerned about progression of future events that do not necessarily follow the evolution of events in the past, and therefore statistical relations cannot be used or formulated. Forward-looking approaches, alternatively, are generally quantitative in nature, relying upon mathematical or statistical representation of the factors being considered, and thereby often use models to derive outputs that reflect how the future may change. Since these techniques use empirical evidence to conceptualize nature into a system governed by a set of rules (i.e. laws of physics, economic functions and identities, etc), these approaches are often very data intensive, requiring large data sets of accurate data with a long enough time series in order to assuage a reasonable understanding of future events.

Compared to qualitative methods, an advantage of quantitative methods is that these can allow one to examine and produce outputs of rates of change in parameters studied. Communication of these results to policy makers, industry, and other stakeholders can benefit decision-making and strategic thinking. A negative attribute however is the inability to include the numerous factors that play a role in economic development and social change that cannot be quantified, such as politics or changes in social values.

Partially for this latter reason, typically a combination of qualitative and qualitative approaches is used in scenario analysis, such as for the scenarios contained in the SRES by the IPCC (IPCC, 2000). Qualitative approaches, in turn, usually at some 
point in time draw upon the quantification of the qualitative data so that outputs can be communicated and shared with necessary stakeholders (ESPON, 2004).

\subsection{Criteria for assessing baseline strengths and weaknesses}

The baseline literature includes several key criteria to help assess the strengths and weaknesses of proposed baseline methods (see table 5). These criteria originate from Annex B and Annex C of the Marrakech Accords and the Marrakech Declaration (UNFCCC, 2001) and are used as a basis to help appraise the baseline methods considered. The criteria established by the UNFCCC were preceded by a number of studies that worked to define, rationalize and prioritize the factors of importance in baseline setting. Ellis and Bosi (1999), for example, emphasized the importance of a baseline being credible, transparent, simple and inexpensive to set up. The authors also noted that the stringency of the baseline approach used directly impacted upon the additionality of the project. A less stringent baseline methodology could result in higher emission reductions than would occur in reality, thereby reducing the environmental effectiveness of the Protocol.

\section{Table 5. Key criteria to evaluate CDM baselines (UNFCCC, 2001)}

Baselines must:

1) Ensure environmental additionality;

2) Provide rigour and accuracy to ensure that net reductions in anthropogenic emissions are real and measurable;

3) Ensure geographical applicability and properly account for spatial scale; 
4) Account for applicable national policies and specific national or regional circumstances, such as sectoral reform initiatives, local fuel availability, power sector expansion plans, and the economic situation in the sector relevant to the project activity;

5) Be transparent and transferable with regard to the choice of assumptions, parameters, and data sources, and;

6) Take account of uncertainties by using conservative assumptions.

Post-Marrakech, these criteria have continued to be rationalized in terms of each criterion's overall importance. Sathaye et al. (2004) suggest that the relative importance of criteria was largely dependent on the type of project being implemented and on the technical characteristics of the power grid into which the project was implemented. Here, criteria related to stringency of the baseline methodology were deemed most important as these had the most influence on maintaining environmental integrity. Lazarus (2003) summarizes criteria established for baseline methodologies drawn from baseline development work completed at the Tellus Institute (for example see Lazarus et al., 2001; Kartha et al., 2002; Kartha et al., 2004). Again emphasized were criteria related to baseline transparency, accessibility, conservativeness, reliability and efficiency.

\subsubsection{The central importance of additionality}

Since the overall objective of the Kyoto Protocol is to lead to a reduction of GHG emissions, the additionality criterion has central importance in terms of what a baseline methodology must strive for. A number of criteria contribute directly to the ability of an MPB producing accurate results in large, dynamic systems such as China's electrical 
sector, although each does so in different ways. These include rigour and accuracy, geographical applicability, and the proper inclusion of national and regional circumstances.

\subsubsection{Rigour and accuracy}

A baseline method that is not accurate will not be able to ensure the successful reduction of GHG emissions from what would have occurred. This criterion is intertwined with other criteria since these feed into the accuracy of the baseline method. This includes how or if regional characteristics or circumstance is considered, and the use of assumptions in any modelling analysis undertaken.

\subsubsection{Geographical applicability and spatial scale}

Spatial scale plays a critical role when using a standardized baseline methodology in the electrical sector. The CDM EB suggest that an electrical system must be defined by the spatial extent of the power plants that can be dispatched without 'significant' transmission constraints, but it must be recognized that in many cases there are significant regional differences in the generation types, vintage and primary sources of different electricity grids across a country. Such characteristics will affect the emission rate of the baseline as interregional flows of electricity can increase or decrease emission rates substantially based on differences in electricity sources in differing regions. These intraregional transfers therefore should ultimately be considered within the baseline methodology. 


\subsubsection{The importance of national and regional circumstances}

If the national or regional energy, economic or environmental policy is expected to change in a way that affects relevant aspects of the electrical sector, then these factors have to be included within the baseline method utilized.

\subsubsection{Data availability and the transparency and transferability of approach used}

Another important attribute is that baselines be 'transparent' and 'transferable'. A baseline methodology must be able to be understood, with the data and techniques utilized accessible so that the baseline can be reproduced. If a baseline or scenario development exercise is highly complex and requires expert knowledge to understand both its formulation and outputs, this diminishes both the applicability of the exercise (as users will be uncertain of the internal workings of the baseline - i.e. the 'black box' syndrome) and the accessibility of the approach. In turn, if a scenario exercise requires significant data or requires monitoring and reporting, this will have a significant effect on the administrative costs of the policy or project. In terms of meeting the objectives of the Kyoto Protocol, lowering the unit cost of baseline production will increase the potential for smaller projects to be undertaken. A methodological approach built upon accessible data will allow others to replicate the baseline or scenario more easily, aiding in evaluation while decreasing the costs of the project. This thereby would increase the effectiveness of the CDM. 


\subsubsection{Uncertainty}

The projection of GHG baselines into the future and the measure of CDM project activity against this baseline is uncertain both because the future direction of the socio-economic drivers leading to GHG emissions are unknown and can interact in unforeseen ways, and due to the counter factual nature of the CDM. Uncertainties also arise due to inadequate scientific understanding of the socio-economic conditions, data gaps or a lack of data and inherent uncertainties of future events in general (Nakicenovic, 2000). 


\section{Using a current perspective as a basis for operationalization}

Synopsis: Can the current status of China's electrical sector give indication of its future development? This question is approached by reviewing the historical progression of both the demand and supply of electricity in China. This review sets the basis for a synopsis to be given of the current state of the sector, allowing for the creation of operating and build margin baselines. These are the most basic of baselines suggested for use by the CDM-EB.

\subsection{Chapter introduction}

This chapter reviews the historical demand and supply of electricity in China as a basis for establishing build and operating margin baselines. Understanding the history of the electrical sector is also important for a number of other reasons. A historical account is used as the basis for one of the methodologies pursued (i.e. factor decomposition). Providing a historical account also is important when envisioning future scenarios, as was explained in the methodology section. Finally, a historical account is important as it can provide the reader a basis of how and why China's electrical sector is as it is currently.

\subsection{The demand and supply of electricity in China}

A pivotal driver of electricity demand in China has been the high rate of economic growth that has occurred over the past twenty years. Industrial output has accelerated driving electricity demand higher, with rates of growth influenced by the structural mix of industrial activity and the consequent relative inputs of capital, labor and energy, the 
relative price of electricity, and the efficiency of energy use. Domestic to global economic processes affect each of these drivers. Alternatively, economic growth has interplayed with electricity demand in the residential (or 'household') sector primarily via processes that have influenced the propensity of households to spend on energy consuming goods, the accessibility to electricity as an energy source, and the cost competitiveness of electricity relative to other fuel types.

\subsubsection{A rapidly growing economy}

China has experienced relatively consistent rapid economic growth since the late 1970s when the country became more open to foreign investment (Ogilvy, 2000). China's GDP grew from 1,365 billion Yuan in 1980 to 9,980 billion Yuan in 2002 (constant 1995 Yuan) - an annual increase of 9.4 percent (CSP, 1998-2004). The majority of growth has occurred within industry. By 2001, this sector had increased its contribution to $52 \%$ from the $38 \%$ of total GDP in 1970 (LBRL, 2004). As its economy has grown, the demand for electricity has risen on average nearly $8 \%$ per annum, higher than the increase for energy in its entirety. This reflects both structural changes towards more electricity intensive economic sectors and processes of electrification within the economy. Demand has accelerated in recent years, and by 2004, total demand surpassed 2,000TWh for the first time (CSPIN, 2006).

A number of factors have characterized China's economic development particularly relevant to electricity demand. These are discussed below. 
Policies to increase industrial output first and a consumer base second

At nearly $40 \%$ of total GDP, China boasts the highest savings rate (i.e., GDP minus consumption as a percentage of GDP) of any country in the world (Dunaway, 2006). A major contributor to this is the household sector: on average, China's 1.3 billion residents save nearly one third of their income per year. Through its influence on the banking sector, over the last twenty years the central government has channelled these enormous savings towards both fixed asset investment and to finance enormous state-owned enterprises (SOEs). Under central planning, industry therefore has developed rapidly, and as a result, industry dominates both energy and electricity demand.

In recent years, China's central government has indicated a policy shift in its economic planning with the goal to increase domestic consumption. The underpinnings of this policy shift are to make the country less reliant on the export market and in-flows of foreign direct investment (FDI) for economic growth, but rather, to encourage domestic-based growth. There are many indications that domestic consumption is already increasing its contribution to the economy - per capita incomes are rising and this is resulting in increased energy and electricity demand in the residential sector (see figure $3)$. 
Figure 3. Changes in electricity demand by sector (1980 and 2002) (LBRL, 2004)

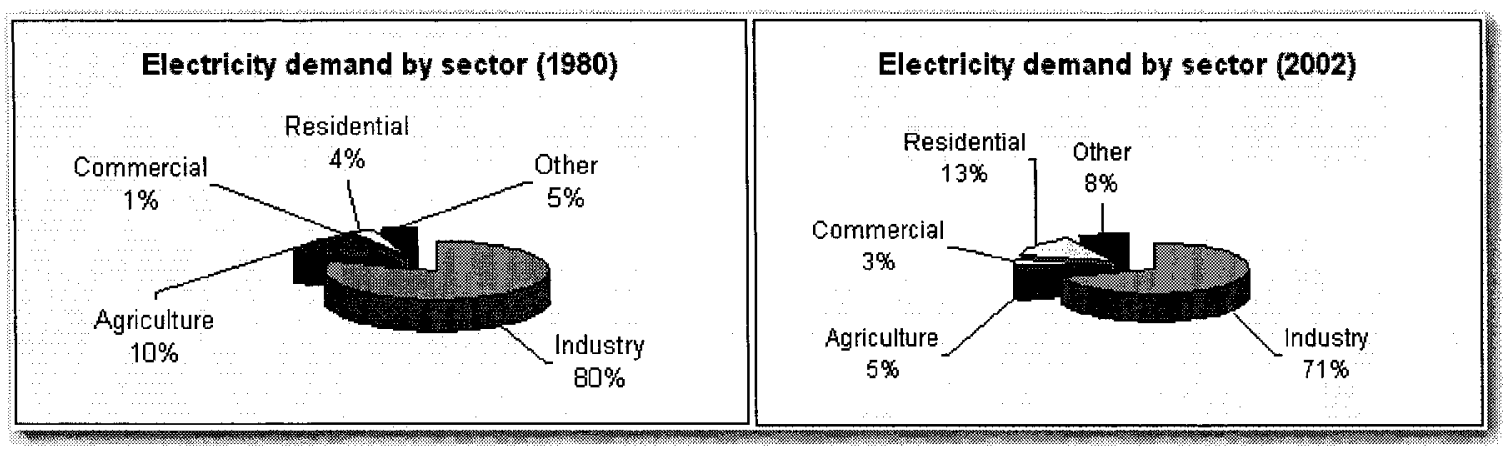

The processes underlying these changes will be important in determining the scale and structure of electricity demand into the future and deserve further discussion and consideration.

\subsubsection{Industrial sector electricity demand}

An important factor that has contributed to the temporal changes in sectoral demand as indicated in figure 3 have been decreases in the energy intensity of industrial output over the last two decades (Zhang, 2003; Wu et al., 2005). Paramount to these trends were changes in the proportion of light to heavy industry and the efficiency of energy use, with this being brought on by both government policy and broader scale processes of globalization.

Industrial restructuring: drivers from the domestic to the global scale

Historically, the majority of China's employment and industrial output has been concentrated in the SOE sector. Many of these enterprises employ in excess of one million people and easily rank amongst the largest corporations in the world but due to both improper incentives and mismanagement, many of these enterprises have also 
operated inefficiently (Chen et al., 2006; Jefferson et al., 2006). Consequently, throughout the 1990's and the early parts of 2000, China's central government undertook a rigorous restructuring and privatization program, with many SOEs converted into limited liability corporations or share-holding enterprises. Since most SOEs are found in heavier and more energy intensive industrial sectors, efforts to improve operational efficiency have led to reductions in energy consumption rates (Steenhof, 2006b). Impacts of this were particularly evident in primary industry and sub-sectors that process raw materials (i.e., chemical production, ferrous metallurgy, etc.) as these sub-sectors are very energy intensive.

Throughout the 1980's and 1990's, structural changes also occurred in terms of relative increases in the output of light industries to heavy industry. Part of this was due to government-led initiatives to restructure the economy towards exports and to increase efficiency in the SOE sector, while part was the result of global economic processes. With the comparative advantage of low wage rates, a large labour force and increasing global flows of capital and investment, output from China's manufacturing sub-sectors surged throughout the 1990's. As these manufacturing sub-sectors are less energy intensive than heavier sub-sectors, this has led to decreasing electricity intensity for industry in its entirety (Steenhof, 2006b). Nonetheless, the structural changes to less energy intensive industries appear to be reversing as in recent years there has been an increase in output from heavy industries (including metallurgy, chemicals, building materials, petrochemicals, and electrical power) as urbanization has increased materials required for infrastructure construction (Shixian et al., 2005). These structural changes 
have accelerated electricity demand in many of China's most industrialized regions, likely contributing to the electricity shortages seen throughout 2003, 2004 and 2005.

\section{Changing energy efficiency of industrial activity}

Policies aimed at increasing energy efficiency through technological or operational changes have also contributed to reductions in the energy intensity of industrial activity. One of the most prominent of these policies was the Energy Conservation Law passed by the State Council in 1997 (Sinton et al., 1998). This not only involved lower-level governments implementing 164 state standards for energy-savings, but also saw the establishment of the China Energy Conservation Investment Corporation, an entity created to help implement and manage energy efficiency and conservation in the industrial sector. Of relevance to electricity consumption, over 600GWh of demand has been targeted so to improve the electrical efficiency of motor systems, pumps, fans and motors (McKane et al., 2005). This electrical equipment contributes upwards of $50 \%$ of electricity consumption in the industrial sector. Efficiency and conservation gains were also made through investments in heat and power cogeneration, district heating, waste heat and gas recovery and other major energy saving projects.

Countering the effects of changes in both efficiency and industrial structure were fuel switches to electricity because of changes in technology. These changes have moved processes formerly powered by coal or other fossil fuels to be powered by electricity. The preference for electricity as an energy substitute due to these technological changes increased electricity demand in the industrial sector by about $20 \%$ in the five years between 1998 and 2002 compared to if these processes did not occur (Steenhof, 2006b). 


\subsubsection{Residential sector electricity demand}

The residential sector's contribution to total electricity demand rose from 4\% in 1980 to $13 \%$ in 2002 (please see figure 3). Increases in residential sector electricity demand are important to consider because most of this consumption occurs during 'peak' hours in the daily load curve when air conditioners, electric appliances, and equipment are run. Peakload requires a more flexible generation unit that, in most countries, consists of natural gas turbines, but in China (due to its wealth of coal generation plants) is largely met using coal-fired, base-load generation units. Any significant increases in electricity demand during these peak hours could therefore have significant impacts upon the supply sector into the future.

Electricity demand in the residential sector is a function of its own price, prices of substitutes, demographics, income, and any policies impacting upon these.

Demographics and population characteristics have played a particularly important role in China over the last number of years owing to the incredible flow of rural-urban migration in the country (Zhang et al., 2003). By 2002, China's urban population was 502 million, with urbanites representing over $39 \%$ of the total national population - a dramatic change from 1980 when less than $20 \%$ of the population lived in urban areas. The movement of people from countryside to city has increased electricity demand as urbanites have better access to electricity, higher incomes and the propensity to spend on electric household appliances and goods.

\section{The effects of changes in per capita income}

As China's economy has accelerated, per capita income has also risen. Between 1990 and 2001, per capita income rose $450 \%$ in urban areas, and $345 \%$ in rural regions (based 
on analysis of data from LBRL, 2004). With the rise in per capita income, the demand for electrical home appliances and equipment (including television sets, washing machines, air conditioners, freezers and refrigerators) has risen rapidly (see table 6) and explains much of the increase in the residential sectors electricity demand that has occurred since 1980.

Table 6. Growth in household appliances in urban China: 1980 - 2002 (derived from LBRL, 2004)

$\begin{array}{lcc} & 1980 & 2002 \\ \text { Televisions } & \text { Units per 100 households } & \\ \text { Clothes washer } & 56 & 141 \\ \text { Refrigerators } & 6 & 93 \\ \text { Air conditioning } & 1 & 87 \\ & 0 & 51\end{array}$

The comparable numbers for rural households show lower penetration rates of household appliances: in 1980, televisions were rare and only a few electric stoves or fridges were owned as many rural households were still using relatively primitive fuels for their daily energy needs. By 2002, for each 100 households there were 108 television sets, 32 clothes washers, and 15 refrigerators.

\section{Substitutes for electricity in the residential sector: home heating}

In the residential sector, electricity is competitive with coal, natural gas, and liquid petroleum gas for home heating. Historically, China's households mostly heated their homes by coal. With escalating air pollution in urban areas and the availability of other fuel substitutes, the use of coal has fallen from nearly $90 \%$ of the residential sector's energy needs to approximately $30 \%$ (based on 2002 data) as electricity has become more widely used for home heating and other energy services. 


\subsubsection{Meeting the demand for electricity and the organization of electricity supply}

With accelerating electricity demand, electricity generation capacity has been rapidly

built in China. Following geographical concentrations of economic activity and electricity demand, the majority of this generation capacity has been developed in the industrialized east, north and southcentral regions. By the end of 2005 , total electricity generator capacity equaled approximately 500GW, 65GW higher than 2004 , and a $730 \%$ rise from the $65 \mathrm{GW}$ installed in 1980 (CSPIN, 2006). Coal powered thermal generation units dominate China's supply mix: in $2005,83 \%$ of capacity was thermal-based, $15 \%$ hydro, $2 \%$ nuclear and renewables. Following the regional distribution of resources available to generate electricity, coal-fired electricity generation dominates northern and eastern regions, while the few areas with higher concentrations of water resources contain higher proportions of hydroelectric capacity (see table 7 and figure 4 ) 
Table 7. Electricity generated by regional grid in China in 2005 (CSPIN, 2006)

\begin{tabular}{|c|c|c|c|c|c|}
\hline \multirow[b]{2}{*}{ Region } & \multicolumn{5}{|c|}{ By source } \\
\hline & $\frac{\text { Total }}{\text { (TWh) }}$ & $\frac{\text { Regional }}{\text { contribution }}$ & Thermal & Hydro & Nuclear \\
\hline Central China & 366 & $15 \%$ & $69 \%$ & $31 \%$ & $0 \%$ \\
\hline Chuanyu Grid & 119 & $5 \%$ & $44 \%$ & $56 \%$ & $0 \%$ \\
\hline East China & 452 & $18 \%$ & $93 \%$ & $2 \%$ & $5 \%$ \\
\hline Fujian Province & 78 & $3 \%$ & $63 \%$ & $37 \%$ & $0 \%$ \\
\hline Guangdong Province & 216 & $9 \%$ & $80 \%$ & $6 \%$ & $14 \%$ \\
\hline Guangxi Province & 42 & $2 \%$ & $57 \%$ & $43 \%$ & $0 \%$ \\
\hline Guizhou Province & 79 & $3 \%$ & $72 \%$ & $28 \%$ & $0 \%$ \\
\hline Hainan Province & 8 & $0.3 \%$ & $89 \%$ & $11 \%$ & $0 \%$ \\
\hline Hong Kong & 43 & $2 \%$ & $100 \%$ & $0 \%$ & $0 \%$ \\
\hline Macao & 2 & $0.1 \%$ & $100 \%$ & $0 \%$ & $0 \%$ \\
\hline North China & 424 & $17 \%$ & $99 \%$ & $1 \%$ & $0 \%$ \\
\hline Northeast China & 191 & $8 \%$ & $93 \%$ & $7 \%$ & $0 \%$ \\
\hline Northwest China & 152 & $6 \%$ & $76 \%$ & $24 \%$ & $0 \%$ \\
\hline Shandong Province & 200 & $8 \%$ & $100 \%$ & $0 \%$ & $0 \%$ \\
\hline Xinjiang Region & 30 & $1 \%$ & $84 \%$ & $16 \%$ & $0 \%$ \\
\hline Yunnan Province & 58 & $2 \%$ & $48 \%$ & $52 \%$ & $0 \%$ \\
\hline Total & 2,459 & $100 \%$ & $83 \%$ & $15 \%$ & $2 \%$ \\
\hline
\end{tabular}


Figure 4. Regional grid map of China (ICF, 2005)

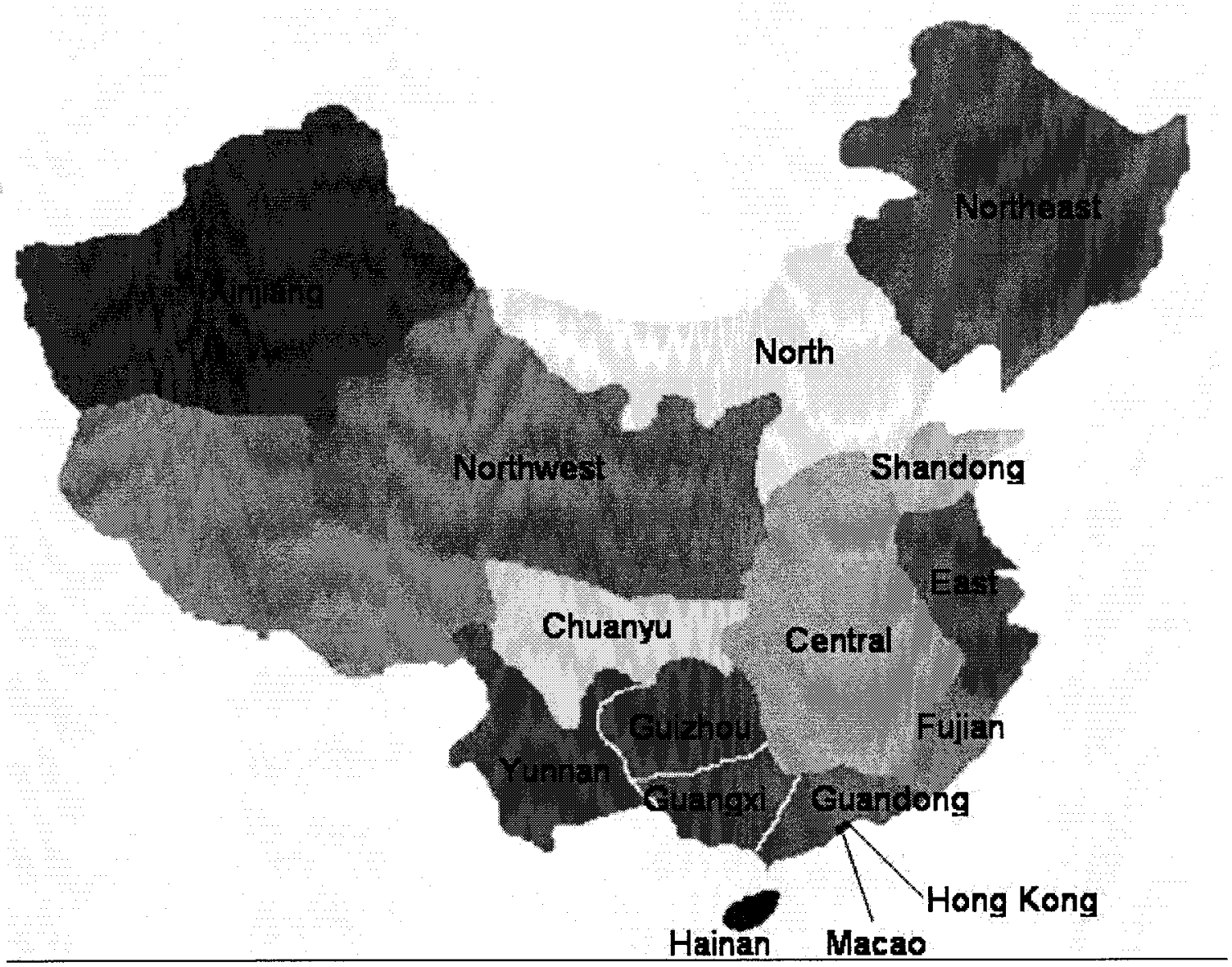

\subsubsection{Changing emission and fuel consumption characteristics}

Coal-fired electricity generation dominates China's electricity supply primarily because coal is the most accessible and inexpensive fuel option available in China and as demand has accelerated, coal-fired electricity generation capacity has been less expensive and easier to build than capital-intensive hydro projects. Capital costs are critical in a rapidly developing economy such as China as adequate financing and investment is often in short supply (Zhang, 2002). Natural gas has played a minor role due to both its expense and unavailability. 
Although the contribution of coal to electricity generation has risen slightly over the past 20 years, there has been a $15 \%$ reduction achieved in the average fuel consumption rate of coal generators, decreasing the emission intensity of electricity provided to end-users in China (Steenhof, 2006a). In 1980, the average thermal generator required approximately 416 grams of standard coal equivalent (gsce) per kWh of electricity generated, but by 2002 , this equaled 356 gsce. Efficiencies have been gained mainly by shutting down small, inefficient generators and are not necessarily tied to the adoption of newer energy efficient technologies. Consequently, the average efficiency of the sector remains below the efficiency seen in developed economies with the possibilities to further increase efficiency tied to the implementation of new, more advanced technologies.

\subsubsection{Increasing interconnections}

China's power industry is progressing into an era of large power networks with the PRC having a long term mandate to have an integrated national electricity network established by 2020 and before then progressively working to integrate individual regional power networks (Jie, 2004). Currently there are seven inter-provincial power grids (i.e. spanning multiple provinces - including East China, Northeast, Central China, North China, Northwest, Chuanyu and South China grids (the South China grid comprises Yunnan, Guizhou, Guangxi and Guandong provincial grids $\left.{ }^{5}\right)$ ) and two independent provincial power networks (Shandong and Fujian). Current interconnections between these regions range in capacity between 600 and 4,500MW, although plans are to build larger transmission capacity in the range of $10,000 \mathrm{MW}$ by 2009 to transfer electricity

\footnotetext{
${ }^{5}$ Guangdong Province provides power to Hong Kong and Macoa
} 
from western hydro rich regions eastwards (ICF, 2004a). This would allow total potential power transfers of a maximum of $88 \mathrm{TWh}$ of electricity per year. There are three non-connected power grids (Hainan, Xinjiang, Xizang (Tibet)).

Interconnections between these regional grids and independent provincial power networks allow more effective use of China's primary resources and reduce regional environmental impacts by allowing cleaner, hydro generated electricity to be transferred into coal dominated areas (Gnansounou et al., 2004). This could impact the emission rates of electricity generation at the regional level in China and affect the amount of CERs generated by CDM project activity in the electrical sector.

\subsection{Establishing operating and build margin baselines}

The first baseline method explored is the use of operating and build margins. This method was introduced and formalized in sub section 3.4.2.

In the sub sections below, first the data available to complete this analysis is described, with this followed by the actual calculation of these baselines. The availability of data is important since this will help determine the feasibility and the level of regional precision possible in the calculation of this baseline method.

\subsubsection{Determination and organization of data}

In order to calculate this baseline method (as formalized in equations 3 and 4), two sources containing information on the operation of power plants across multiple years (including electricity generated by source, fuel type, and the fuel consumption rates of thermal plants) were used. 
First, data from the China State Power Information Network (CSPIN) provided provincial level annual electricity generation statistics by generation source (i.e. hydro, thermal, nuclear) for the most recent year (2005) (CSPIN, 2006). The CSPIN is an information dissemination point established by the government of China after the devolvement of the State Power Corporation in 2002. Secondly, the China Power Statistical Yeakbook provided comparable provincial level information for earlier years (which in this case is for 2002) in addition to fuel consumption rates for coal powered generators (CEPB, 2004). There were no sources available that provided data for 2003 and 2004. Furthermore, no data was located for the Tibet region so this region was not included in the analysis.

\subsubsection{Calculation of the operating and build margin emission baseline}

The results of the OM and BM baseline analysis were computed for the 30 provinces and autonomous regions in China (see figure 5). ${ }^{6}$

\footnotetext{
${ }^{6}$ Electricity statistics for Hong Kong and Macao have been included in the Guangdong Provincial data
} 
Figure 5. Operating and build margin baselines (provincial level).

NC $=$ North China Grid, NE $=$ Northeast China Grid, E $=$ East China Grid, C $=$ Central China Grid, Sh = Shandong Provincial Grid, S = South China Grid (S), Ch = Chuangyu Provincial Grid, Yunnan

Provincial Grid (Y), NW = Northwest China Grid, and X = the Xingjiang Grid.

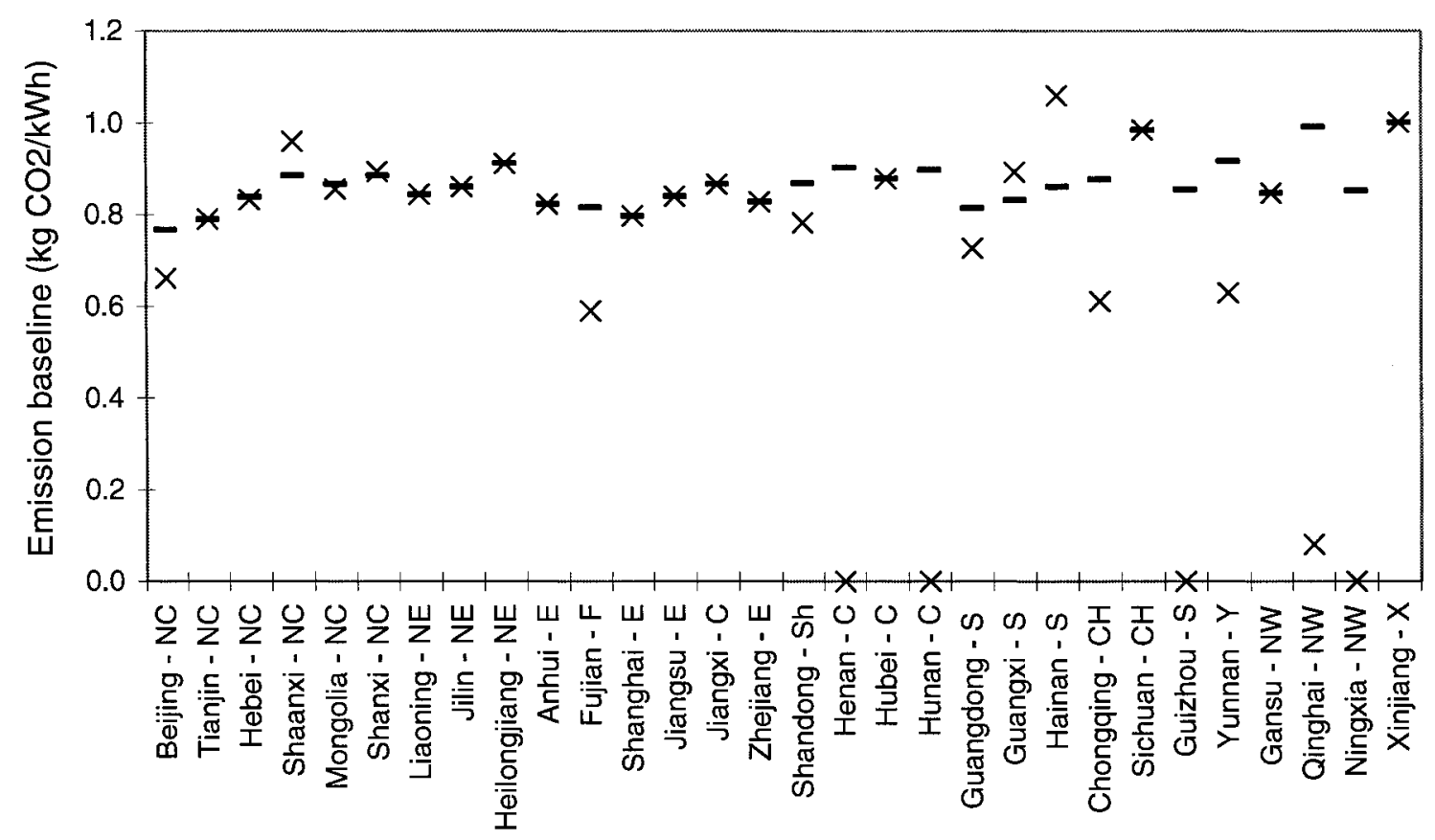

\section{- Operating margin (2005) $\times$ Build margin (2002-05)}

A number of key points are noted concerning the provincial variation of the emission baseline:

- Differences in the efficiency of coal generation plants explain the variation in the OM baseline - grids containing more efficient plants have a lower baseline. Here, a strong positive relationship exists between these and the extent of industrialization of each province: Beijing, one of China's more industrialized and prosperous regions, boasts the most efficient thermal generation units (319 grams 
of standard coal equivalent (gsc)/KWh) compared to the relatively underdeveloped northwestern provinces of Qinghai and Xinjiang where efficiencies are 412 and $416 \mathrm{gsc} / \mathrm{kWh}$ respectively;

- The provincial variation of the BM baseline can be explained by differences in the ratio of fossil fuel-fired to non-fossil fuel-fired electricity: regions that have seen a greater proportion of nuclear or hydro-based electricity come online in recent years experience a lower BM baseline. Generally, this follows historical configurations of generation source mix - regions dominated by non-fossil fuelfired electricity have a lower baseline than others.

- A number of southcentral provinces experienced a more pronounced fall in the BM baseline, including Shanghai which saw this decrease from 0.814 to 0.412 $\mathrm{kgCO}_{2} / \mathrm{kWh}$ and Henan which saw its baseline decrease to zero. These decreases relate to the Three Gorges dam project where large regional transfers of electricity acted to decrease GHG emissions.

Finally, it is important to highlight the variation in the provincial emission baselines because these variations help demonstrate the potential error that could be introduced by using national level emission baselines (see table 8 ). The fuel consumption rate for thermal generators is included in the baseline since this is an important determinant in the emission rates calculated. 
Table 8. Summary of statistical variation in provincial level emission baselines

$\begin{array}{lrrrr} & & & & \begin{array}{c}\text { Standard } \\ \text { deviation }\end{array} \\ \text { OM (2005) (kgCO2/kWh) } & \text { Average } & \text { Maximum } & \text { Minimum } & \text { Mat } \\ \text { BM (2002-05) (kgCO2/kWh) } & 0.87 & 1.00 & 0.77 & 0.06 \\ \text { Fuel consumption rate } & 0.69 & 1.06 & 0.00 & 0.33 \\ \text { (gsce/kWh) } & 360.10 & 416.00 & 319.00 & 22.99\end{array}$

The OM has the highest average baseline, while the BM baseline has the lowest. This reflects that more hydro-based electricity generation came on line over the past three years than compared to the historical average. Meanwhile, there is general consistency in the maxima (since an emission intensity of $1 \mathrm{~kg}$ of $\mathrm{CO}_{2}$ per $\mathrm{KWh}$ reflects electricity that is nearly $100 \%$ generated from coal) but a large standard deviation in minima. The reason is that when only considering the most recent generation activity (as is the case in the BM baseline) the baseline minima reflects that in some provinces all new demand has been met by hydro generation units or had increases in nuclear generation capacity, while other regions have continued to rely on coal. The statistical range in the average fuel consumption rate of thermal generators in China reflects that there is a range in average efficiencies of generators across China.

The statistical variation of these baselines helps emphasize that significant errors could be introduced if national level data was used rather than provincial level data, and therefore help illustrate a strength of this approach in terms of its ability to consider regional detail. 


\subsubsection{Regional aggregation of provincial level data}

The above analysis has provided insight on the operating and build margins baselines at the provincial level. However, as was previously discussed, China is now moving to a system of larger regional grids. Therefore, it is appropriate to show regionally derived emission baselines. Provincial level data is therefore grouped into the regional grids that exist in China (see figure 6). For illustrative purposes, the source contribution of each region is also shown.

Figure 6. Operating and build margins (regional level)

All grid names and short forms were defined in figure 5 .

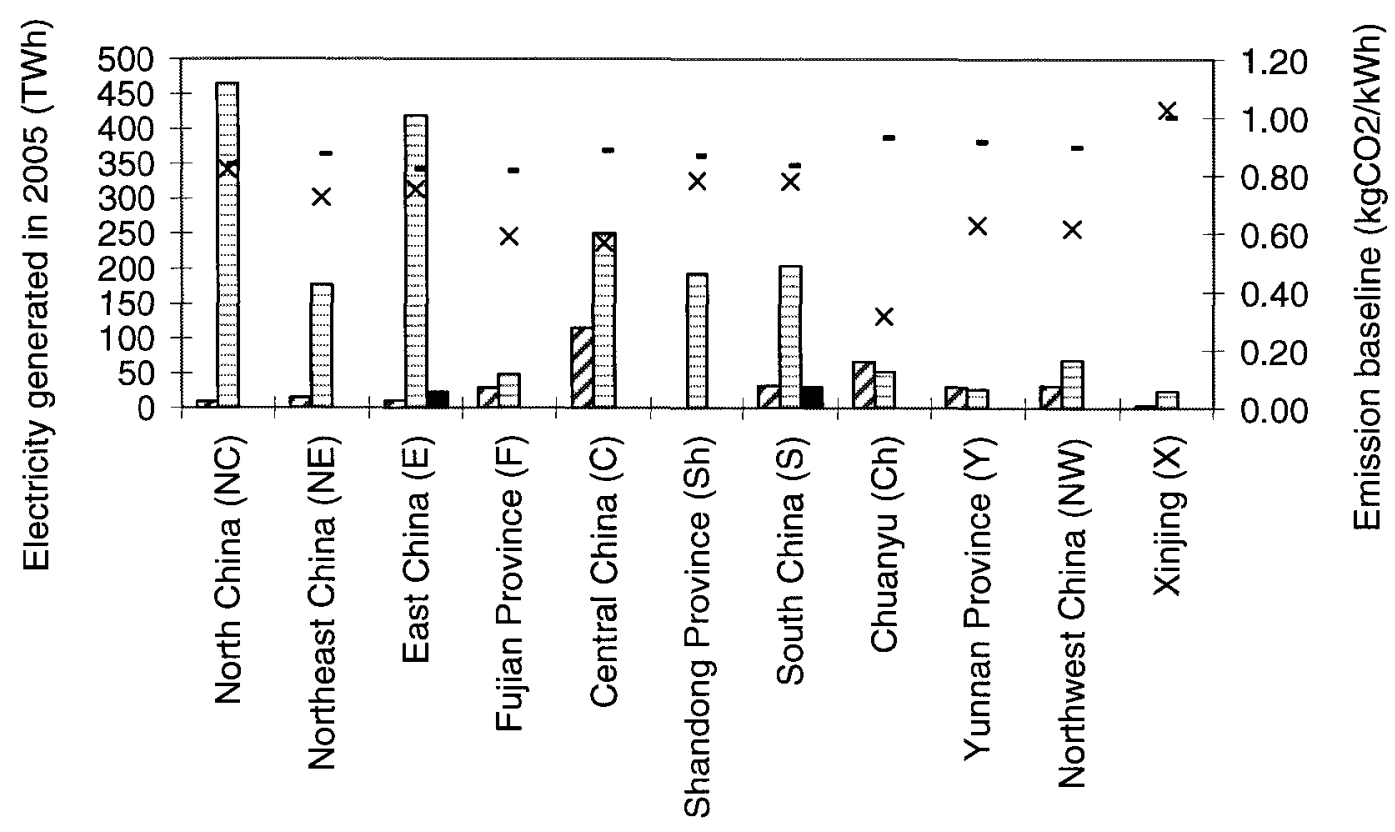

$\square$ Hydro generation (2005) $\square$ Thermal generation (2005) Nuclear generation (2005)

- Operating margin (2005) × Build margin (2002-05) 
Several key points are illustrated by figure 6 . First, although the northern region of China (which contains most of China's heavy industry) has the most thermal generated electricity, the average efficiency of thermal generators is higher than other regions, resulting in a lower OM emission baseline than other regions. Meanwhile, the continuing high contribution of coal to its electricity mix results in a higher BM emission baseline than the other regions. The region with highest OM baseline is the Xinjing region in far northwest China. This is a much less economically developed region than in the east, and consequently has some of the most inefficient generating capacity in the country.

A few other differences emerge when comparing the regional and the provincial resolution data. With the former, it is more readily apparent that the environmental characteristics of electricity generation have improved in the southcentral region of China. This is explained largely by the coming online of major hydroelectric projects such as the Three Gorges. It is also apparent, however, that the environmental characteristics of new generation have actually worsened in the rapidly growing eastern region. The reason for this is that coal has largely met the rapidly increasing electricity demand in this region (CSPIN, 2006).

Now turning to the statistical variation of the regional variation, again, the average, maximum, minimum and standard deviation are provided for the various baseline methods and for the fuel consumption rate (see table 9):

Table 9. Summary of statistical variation in regional level emission baselines

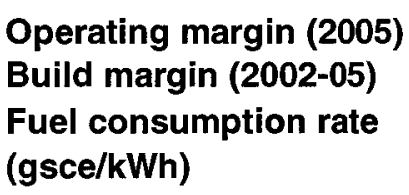

Operating margin (2005) Bulld margin (2002-05) (gsce/kWh)

$\begin{array}{rrrr}\text { Average } & \text { Maximum } & \text { Minimum } & \begin{array}{r}\text { Standard } \\ \text { deviation }\end{array} \\ 0.85 & 0.89 & 0.82 & 0.03 \\ 0.71 & 0.82 & 0.57 & 0.10 \\ & & & \\ 365.86 & 416.00 & 339.00 & 22.84\end{array}$


There is a small standard deviation in the OM baseline but a large standard deviation for the BM. This is especially the case for the 2002-05 block of generation data. The reason for this is the large amounts of hydroelectricity that displaced thermal electricity in the southwest region of China during this time. 


\section{Using decomposition analysis as a basis for operationalization}

Synopsis: Decomposition analysis has been proposed as an alternative method for baseline analysis and has been described in the methodology section as a technique used primarily in the past for analysis in the demand sector. However, since emission baselines in the electrical sector are uniquely tied into both electricity demand and supply, decomposition can be used in the analysis of both. In this chapter, analytical exercises directed at both the demand and supply sides of the electrical sector are undertaken, with the fundamental question pursued being if historical trends, and the quantification of factors that contribute to these, can give indication of future occurrences.

\subsection{Chapter introduction}

Decomposition analysis is a tool which can help quantify the impacts of major macroeconomic processes such as changes in economic structure, efficiency, or economic output on energy consumption and/or emission levels, and consequently it has been widely utilized in the environmental and energy literature to help illustrate the effects of influential policies acting upon these parameters. These are reasons why this form of analysis may be applicable as a baseline tool for CDM projects. Determining the contribution of factors operating historically to changes in both electricity demand and the GHG emissions resulting from electricity generation may help illustrate what factors are most important and the effects of broad-based policy decisions or economic processes. 
In this chapter decomposition is therefore used to study both electricity demand and the progression of factors that have contributed to the emissions resulting from the electricity generated in China. This analysis forms the basis for the application of decomposition in the forward-looking scenarios contained in later chapters.

\subsection{Using decomposition in the electricity demand sector}

Decomposition analysis is used to understand changes in electricity demand resulting from changes in industrial activity in China for the years spanning from 1998 to 2002. Industry contributes upwards of $70 \%$ of the nation's total electricity demand, and therefore a focus upon this sector is useful. Furthermore, there are a number of potential demand side CDM projects that could take place in the industrial sector related to electricity use, such as initiatives to increase end-use efficiency or to switch fuels. Therefore, such analysis could help inform both the policy and economic context into which potential CDM projects might take place.

In order to study electricity demand in China's industrial sector, a decomposition technique proposed by Park (1992) and later modified by Zhang (2003) is applied. Park (1992) initially proposed the general decomposition technique used (the Laspeyres technique), and Zhang (2003) adapted and applied this approach to help understand changes in total energy demand in China. It is further adapted and applied in a way suitable for understanding electricity demand - primarily by introducing terms that can better account for changes in the efficiency of electricity use and fuel switching effects. Using this decomposition approach, changes in four economic processes specific to the industrial sector are investigated: the role of increasing industrial activity (by value added at the sub-sector level), shifts in economic activity towards industrial sectors more or less 
heavily weighted by electricity use, fuel shifts toward electricity, and changes in energy intensity. Data available for 1998 through till 2002 allows a detailed analysis to be undertaken of 37 different industrial sub-sectors, ranging from those at the primary level (i.e. coal mining, oil and gas extraction, etc.) and secondary sub-sectors (i.e. chemical industries, cement production, manufacturing, etc.).

This research is useful to both policy makers and analysts concerned with emerging energy issues in China, and specifically those interested in future directions of electricity demand in China and the role of China's rapidly growing industrial sector within this.

\subsubsection{Methods and data}

The basic premise of decomposition analysis for the purposes of understanding changes in energy demand is represented by the following identity:

$$
\Delta E_{\text {Tot }}=\Delta E_{\text {oUT }}+\Delta E_{\text {Str }}+\Delta E_{\text {Int }}
$$

Where:

$\Delta \mathrm{E}_{\mathrm{Tot}}=$ total change in energy demand;

$\Delta \mathrm{E}_{\mathrm{out}}=$ change in energy demand due to changes in activity;

$\Delta \mathrm{E}_{\mathrm{str}}=$ change in energy demand due to structure, and;

$\Delta \mathrm{E}_{\mathrm{int}}=$ changes in energy demand due to intensity . 
Using the Laspeyres decomposition method, activity, structure and intensity can be decomposed using the following formulae:

$$
\begin{aligned}
& \Delta E_{\text {out }}=\sum_{i} Q_{t} S_{i, 0} I_{i, 0}-E_{0}=\left(Q_{t}-Q_{0}\right) \sum_{i} S_{i, 0} I_{i, 0} \\
& \Delta E_{s t r}=\sum_{i} Q_{t} S_{i, t} I_{i, t}-E_{0}=Q_{0} \sum_{i}\left(S_{i, t}-S_{i, 0}\right) I_{i, 0} \\
& \Delta E_{\mathrm{int}}=\sum_{i} Q_{t} S_{i, 0} I_{i, t}-E_{0}=Q_{0} \sum_{i}\left(I_{i, t}-I_{i, 0}\right) S_{i, 0}
\end{aligned}
$$

Where:

$\mathrm{Q}_{0}$ and $\mathrm{Q}_{\mathbf{t}}=$ aggregate production in the industrial sector in year 0 and year $\mathrm{t}$ in million Yuan;

$\mathrm{S}_{\mathrm{i}, 0}$ and $\mathrm{S}_{\mathrm{i}, \mathrm{t}}=\mathrm{i}^{\text {th }}$ industrial sub-sector's share of aggregate production in year 0 and year $\mathrm{t}$, and;

$\mathrm{I}_{\mathrm{i}, 0}$ and $\mathrm{I}_{\mathrm{i}, \mathrm{t}}=$ electricity intensity in each industrial sub-sector $\mathrm{i}$ in year 0 and year $\mathrm{t}$ in TWh per million Yuan.

The basic idea behind each of these three formulae is to determine changes resulting from one factor by allowing this to change with time, while holding all others constant. However, each of these will typically leave a residual, and therefore Zhang (2003) 
proposed and used a new method that did not. He did this by keeping the definition of the first term unchanged but redefining the last two terms. Changes in structure were accounted for by differences between what energy consumption would have been if each sub-sectoral activity at year $t$ had been produced at the energy intensity of year 0 and if the aggregate production at year $t$ had been composed in the same way as at year 0 . Changes in intensity represented the difference between the observed energy consumption and what energy consumption would have been if each sub sectoral activity at year $t$ had been produced at the energy intensity of year 0 . These changes can be summarized as follows:

$$
\begin{gathered}
\Delta E_{s t r}=\sum_{i}\left(Q_{t} S_{i, t} I_{i, t}-Q_{t} S_{i, 0} I_{t, t}\right)=Q_{t} \sum_{i}\left(S_{i, t}-S_{i, 0}\right) I_{i, 0} \\
\Delta E_{\mathrm{int}}=\sum_{i}\left(Q_{t} S_{i, t} I_{i, t}-Q_{t} S_{i, t} I_{i, 0}\right)=Q_{t} \sum_{i}\left(S_{i, t}\left(I_{i, t}-I_{i, 0}\right)\right.
\end{gathered}
$$

A unique and beneficial aspect of decomposition analysis is that analysis and results can be undertaken and presented using either an additive or a multiplicative technique. Showing results using both forms is advantageous for presentation as either one might be more easily understood depending on the aptitude of the reader in using absolute differences or percentage changes. The decomposition equations given previous have all taken the additive form of decomposition and therefore their results show changes in absolutes. In order to capture relative rather than absolute change, these can be modified to the multiplicative form simply by introducing divisors into the equation where subtraction was previously used. The results therefore will be given as a 
percentage rather than an absolute change. These modifications are enacted for activity, structure and intensity as follows:

$$
\begin{gathered}
P E_{\text {out }}=\sum_{i} Q_{t} S_{i, 0} I_{i, 0} / E_{0} \\
P E_{s t r}=\sum_{i}\left(Q_{t} S_{i, t} I_{i, t} / Q_{t} S_{i, 0} I_{t, t}\right) \\
P E_{\mathrm{int}}=\sum_{i}\left(Q_{t} S_{i, t} I_{i, t} / Q_{t} S_{i, t} I_{i, 0}\right)
\end{gathered}
$$

Where:

$\mathrm{PE}=$ the percentage change in each factor being decomposed.

Other terms are defined for equations 9 and 10.

Although Zhang's (2003) earlier work was not focused upon the electrical sector but rather energy use in its entirety, with a few modifications, the decomposition equations surveyed above can be extended for the purposes of this dissertation. Because ' $\mathrm{I}$ ' is a measure of intensity per value added, changes in this may reflect both actual physical increases in energy efficiency and/or fuel switches between electricity and substitute forms of energy. Therefore, in order to account for this, ' $I$ ' is further decomposed into the components of actual energy efficiency and any fuel switching to electricity from alternative energy types. This is done by calculating the amount of 
additional energy resulting from fuel shifts to or away from electricity. This amount can then be subtracted from the total intensity value so to leave the amount attributable to real energy efficiency changes. This step is summarized by the following equation:

$$
\Delta E_{\text {FuelShare }}=\sum_{i}\left(E F_{i, t} E_{i, 0}-E F_{i, 0} E_{t, 0}\right)=\sum_{i}\left(E F_{i, t}-E F_{i, 0}\right) E_{i, 0}
$$

Based on the above, changes in real electricity intensity can be calculated as:

$$
\Delta E_{e f f}=\left(\sum_{i}\left(Q_{t} S_{i, t} E I_{i, t}-Q_{t} S_{i, t} E I_{i, 0}\right)=Q_{t} \sum_{i}\left(S_{i, t}\left(E I_{i, t}-E I_{i, 0}\right)\right)-\Delta E_{\text {FuelShare }}\right.
$$

Where:

$\Delta \mathrm{E}_{\text {FuelShare }}=$ change in electricity due to fuel shifts;

$\mathrm{EF}=$ proportion of electricity to total energy;

$\mathrm{E}=$ total energy demand;

$\mathrm{EI}=$ energy efficiency, and;

$\Delta \mathrm{E}_{\text {eff }}=$ real changes in energy efficiency .

The focus on the years spanning 1998 to 2002 largely reflects the availability of data.

Before 1998, only aggregated data for light and heavy industry was available, whereas a more detailed data set for 37 sub-sectors was available for the years following. 


\subsubsection{Empirical data used}

In order to help illustrate how equations 7 through 17 are utilized, a brief overview is presented of the empirical data used.

Electricity demand and industrial activity data existed for 37 industrial sub-sectors from China's Statistical Yearbook as published in China's Statistical Yearbook (CSP, 1998-2004). These industrial sub-sectors were first categorized into three classes in order to aid in the presentation and interpretation of the data: primary $(\mathrm{P})$, secondary processing (S-P), and secondary - manufacturing (S-M). ${ }^{7}$ 'P' sub-sectors include primary resources such as coal and non-mineral mining, petroleum and natural gas extraction, etc. Secondary 'S-P' sub-sectors include those that process primary resources for manufacturing, such as smelting and pressing of mineral products and production of chemicals, while 'S-M' sub-sectors involve the manufacturing of products suitable for end-use consumption from the materials produced in earlier economic processes.

The value added of each industrial sub-sector is used as an indicator of economic activity. Using gross activity in the analysis would risk double counting industrial activity as activity in one sector may correspond to the same activity already counted in another sector. All energy data used is in energy equivalency - e.g. electricity demand is given in kilowatt-hours or a multiple of this. Table 10 lists the 37 industrial sub-sectors used in the analysis, their categorization, as well as presenting their value added and electricity demand for 2002 and 1998.

\footnotetext{
${ }^{7}$ These latter industrial classes include those sub-sectors that refine primary products for manufacturing (i.e. 'Secondary - processing'), and those sub-sectors that manufacture products from this (i.e. Secondary manufacturing')
} 
Table 10. Empirical data used to study industrial sector electricity demand (data from China Statistical Yearbook: 1998 - 2002)

$\mathbf{P}=$ Primary, $\mathrm{S}-\mathrm{M}=$ Secondary - manufacturing, $\mathrm{S}-\mathrm{P}=$ Secondary - processing.

\begin{tabular}{|c|c|c|c|c|c|}
\hline \multirow[b]{2}{*}{ Sector } & \multirow[b]{2}{*}{ Category } & \multicolumn{2}{|c|}{$\begin{array}{l}\text { Demand } \\
\text { (TWh) }\end{array}$} & \multicolumn{2}{|c|}{$\begin{array}{l}\text { Value added } \\
\text { (million Yuan) }\end{array}$} \\
\hline & & 2002 & 1998 & 2002 & 1998 \\
\hline Production \& Supply of Gas & $\mathrm{P}$ & 37 & 26 & 53 & 14 \\
\hline Logging \& Transport of Timber \& Bamboo & $\mathrm{P}$ & 11 & 11 & 56 & 82 \\
\hline Ferrous Metals Mining \& Dressing & $\mathrm{P}$ & 76 & 57 & 86 & 54 \\
\hline Nonmetal Minerals Mining \& Dressing & $\mathrm{P}$ & 96 & 60 & 142 & 111 \\
\hline Nonferrous Metals Mining \& Dressing & $\mathrm{P}$ & 88 & 66 & 151 & 111 \\
\hline Production \& Supply of Tap Water & $\mathrm{P}$ & 140 & 129 & 171 & 124 \\
\hline Coal Mining \& Dressing & $\mathbf{P}$ & 499 & 424 & 919 & 602 \\
\hline Raw Chemical Materials \& Products & $\mathrm{P}$ & 1,356 & 1,053 & 1,863 & 1,103 \\
\hline Petroleum \& Natural Gas Extraction & $\mathrm{P}$ & 350 & 295 & 1,937 & 1,186 \\
\hline Production/Supply of Electricity \& Steam & $\mathrm{P}$ & 2,477 & 1,646 & 3,166 & 1,875 \\
\hline Furniture Manufacturing & S-M & 11 & 9 & 139 & 77 \\
\hline Cultural, Educational \& Sports Goods & S-M & 32 & 16 & 205 & 141 \\
\hline Instruments, Cultural \& Office Supplies & S-M & 32 & 18 & 269 & 168 \\
\hline Printing \& Record Medium Reproduction & S-M & 34 & 24 & 280 & 183 \\
\hline Rubber Products & S-M & 109 & 81 & 293 & 203 \\
\hline Leather, Furs, Down \& Related Products & S-M & 36 & 24 & 458 & 273 \\
\hline Food Manufacturing & S-M & 114 & 86 & 554 & 325 \\
\hline Papermaking \& Paper Products & S-M & 285 & 184 & 571 & 319 \\
\hline Plastic Products & S-M & 144 & 98 & 647 & 354 \\
\hline Beverage Manufacturing & S-M & 68 & 58 & 710 & 544 \\
\hline Garments \& Other Fiber Products & S-M & 59 & 40 & 746 & 482 \\
\hline Special Purpose Equipment & S-M & 103 & 79 & 782 & 485 \\
\hline Medical \& Pharmaceutical Products & S-M & 98 & 77 & 835 & 433 \\
\hline Metal Products & S-M & 282 & 143 & 841 & 504 \\
\hline Ordinary Machinery & S-M & 202 & 156 & 1,153 & 697 \\
\hline Nonmetal Mineral Products & S-M & 880 & 649 & 1,365 & 909 \\
\hline Textile Industry & S-M & 454 & 331 & 1,567 & 1,017 \\
\hline Electric Equipment \& Machinery & S-M & 130 & 79 & 1,585 & 880 \\
\hline Transport Equipment & S-M & 259 & 202 & 2,177 & 1,080 \\
\hline Electronics \&Telecommunications & S-M & 150 & 85 & 2,521 & 1,121 \\
\hline Timber Processing & S-P & 38 & 24 & 214 & 113 \\
\hline Chemical Fiber & S-P & 206 & 150 & 249 & 185 \\
\hline Smelting \& Pressing of Nonferrous Metals & S-P & 824 & 539 & 626 & 332 \\
\hline Petroleum Processing \& Coking & S-P & 331 & 192 & 1,004 & 529 \\
\hline Food Processing & S-P & 195 & 191 & 1,113 & 682 \\
\hline Tobacco Processing & S-P & 31 & 22 & 1,360 & 886 \\
\hline Smelting \& Pressing of Ferrous Metals & S-P & 1,323 & 937 & 1,799 & 983 \\
\hline
\end{tabular}


A brief mention must be given of the quality of the data used in this analysis and how this affected the analysis completed. Sinton and Fridley (2003) writes about the quality of China's economic and energy data, particularly that this might contain inaccuracies that make trends in energy efficiency appear more pronounced and economic growth stronger. Nonetheless, although these data issues have been identified in the past, they likely have a minimal impact on the analysis completed for this article when considering the short time period under investigation (i.e. the five years between 1998 and 2002).

\subsubsection{Analyzing electricity demand in China's industrial sector}

The four factors investigated in the context of changes in overall electricity consumption and its intensity per unit of economic activity are first defined. Total activity, the first factor investigated, is simply a measure of changes in total industrial activity as measured by value added. The second term investigated, electricity intensity, is measured by the amount of electricity consumed per unit of value added. Across the aggregate of industry, this term reflects two underlying processes: real energy efficiency gains, or actual physical increases in energy efficiency associated with the technical characteristics of the electrical equipment being run (including fans, compressors, electric furnaces, etc.) and the composition of industrial sub-sectors operating within. In terms of the latter, electricity intensity will be high if the share of electricity intensive sub-sectors increases relative to less electric intensive sub-sectors, and vice versa. This effect of changes in the contributions of the individual sub-sectors to total activity is often referred to as structural change, and is the third term investigated. Of the individual industrial sub-sectors, electricity intensity is highest for primary industries involved in the extraction and 
processing of primary commodities, such as the smelting and pressing of ferrous and nonferrous metals, production and supply of gas, chemical fiber, etc, while manufacturing sub-sectors, such as textiles, furniture manufacturing and electronics, require less electricity per unit of economic activity. Therefore, a shift to these latter would act to decrease aggregate electricity intensity.

A fourth factor, fuel switches, can also lead to changes in overall electricity demand and intensity. Since 1980 , the contribution of electricity to total energy use in China's industrial sector has increased from $24.3 \%$ to $43.6 \%$. Both price and technological factors have contributed to this. When the price of competitive fuels to electricity increase, electricity demand will likely increase as a result, while as industrial sectors modernize, processes originally using thermal power might convert to electrical power. For example, in the steel production sector modern and technologically advanced electric arc furnaces (used to produce steel from scrap material) could increase proportionally at the expense of more energy intensive, coal-based blast furnaces. Currently $92 \%$ of the steel produced in China is from these blast furnaces, which is high when compared to both the international average of $68 \%$ and other developing countries as well (IISI, 2006). India, for example, produces $85 \%$ of its coal using blast furnaces, while Taiwan, even if in later stages of development, uses blast furnaces for $54 \%$ of steel production. However, the fuel switching effect, as it is often termed, often interacts with structural effects and therefore this factor can be somewhat arduous to isolate. As such, care and attention should be given to the results of any associated analysis. 


\subsubsection{Previous studies on energy demand in China's industrial sector}

There have been a number of studies in the literature investigating factors influencing aggregate energy consumption in China's industrial sector, but there have been few, if any, investigating trends in electricity consumption within China's industry or industrial sub-sectors. Nonetheless, a brief review of these former studies is beneficial seeing the similarities between energy and electricity demand.

Evaluating industry energy intensity decreases over the 1980's, Sinton and Levine (1994) suggested that real intensity changes resulted in approximately $70 \%$ of the overall energy intensity decrease experienced in the industrial sector during this period, while structural changes contributed to approximately $30 \%$. A common margin of error of ten percent was suggested for each of these. A similar conclusion was made by Garbaccio et al. (1999) for the 1987 to 1992 period, where they concluded that the fall in energy use during that time was mostly due to a fall in real energy intensity. For this, they drew upon input-output tables for the period from 1987 to 1992 to decompose a range of technical and structural change indicators, including changes in the quantity and composition of imports and exports. In addition to determining that real intensity decreases was the major causal factor in leading to overall energy decline, they found that structural changes within the industrial sector actually increased the use of energy over this period. Zhang (2003) studied the energy use and industrial value added of 29 industrial sub-sectors for the 1990's and similar to the earlier periods analyzed in the aforementioned literature sources, found that the major contributor to the decline in industrial energy use during this later time was a decline in real energy intensity. This 
conclusion helped to establish that the trends of real energy intensity declines seen in the 1980s were maintained throughout the 1990s.

There have been some dissenting conclusions to the view that real energy intensity changes were the main determinant in influencing energy demand. For example, Sinton and Levine (1994), suggest that structural shifts away from more energyintensive industrial sub-sectors have been a much more significant causal factor. A similar conclusion was reached by Fisher-Vanden et al. (2004) who completed multiple levels of decomposition analysis in China's industrial sector, including analysis completed at the firm level. Here the Divisia decomposition technique was used to study the roles of real intensity decreases, structural change, increasing energy prices, research and development expenditures, as well as ownership reform in the enterprise sector on observed decreases in overall energy intensity. From this it was concluded that structural changes and decreases in real energy intensity contributed nearly equally to falls in overall energy intensity.

An issue that has emerged in using aggregate data to determine the impact of factors contributing to changes in energy demand is that this can minimize the effects of structural changes while exaggerating the effects of intensity changes. This has been identified by a number of authors (e.g., Sinton and Levine (1994), Garbaccio, Ho and Jorgenson (1999), and Fisher-Vanden et al (2004)) and should be acknowledged as a point of uncertainty when using results from methods such as decomposition analysis.

Although there have been these above examples in the literature examining energy use in China's industrial sector, there are no examples which have applied decomposition analysis to understand changes in industrial sector electricity demand. 
This is an important contribution as understanding changes in industrial sector electricity demand may illustrate to policy makers and analysts important factors that affect electricity demand, and consequently, may provide a better understanding of how electricity demand (and required capacity) may change into the future.

\subsubsection{Illustrating key trends influencing industrial sector electricity demand}

Table 11 summarizes key trends influencing industrial sector electricity demand as derived from the empirical data included in table 10 :

Table 11. Key trends influencing industrial sector electricity demand

\begin{tabular}{|c|c|c|c|c|c|c|c|c|}
\hline \multirow[b]{2}{*}{ Sector } & \multicolumn{4}{|c|}{1998} & \multicolumn{4}{|c|}{2002} \\
\hline & $\begin{array}{c}\text { Electricity } \\
\text { demand } \\
\text { (TWh) }\end{array}$ & $\begin{array}{c}\text { Value } \\
\text { added } \\
\text { (million } \\
\text { Yuan) }\end{array}$ & $\begin{array}{c}\text { Structure } \\
(\%)\end{array}$ & $\begin{array}{c}\text { Electricity } \\
\text { intensity } \\
\text { (TWh/mill. } \\
\text { Yuan) }\end{array}$ & $\begin{array}{c}\text { Electricity } \\
\text { demand } \\
\text { (TWh) }\end{array}$ & $\begin{array}{c}\text { Value } \\
\text { added } \\
\text { (million } \\
\text { Yuan) }\end{array}$ & $\begin{array}{c}\text { Structure } \\
(\%)\end{array}$ & $\begin{array}{c}\text { Electricity } \\
\text { intensity } \\
\text { (TWh/mill. } \\
\text { Yuan) }\end{array}$ \\
\hline Primary & 377 & 5,263 & 0.27 & 0.07 & 513 & 8,544 & 0.26 & 0.06 \\
\hline Secondary - processing & 205 & 3,708 & 0.19 & 0.06 & 295 & 6,365 & 0.20 & 0.05 \\
\hline Secondary - manufacturing & 244 & 10,196 & 0.53 & 0.02 & 348 & 17,695 & 0.54 & 0.02 \\
\hline Total & 826 & 19,167 & 1.00 & 0.04 & $\overline{1,156}$ & 32,604 & 1.00 & 0.04 \\
\hline
\end{tabular}

- The most significant of efficiency gains between 1998 and 2002 occurred in the manufacturing sub-sectors, which saw a decrease in electricity intensity of nearly $18 \%$.

- Overall, there was a shift in industrial structure to a heavier weighting in manufacturing industries versus more energy intensive primary industries.

- There was rapid growth in the manufacturing sub-sectors between 1998 and 2002, particularly in such sub-sectors as electronic and 
telecommunications equipment (activity growth of 148\%) and transport equipment (activity growth of $133 \%$ ).

- There was slower growth in primary resource sub-sectors such as mining of non-minerals (45\% growth) and oil extraction (29\% growth).

- There were significant fuel shifts towards electricity over this period. Electricity contributed $9 \%$ of total energy end use in 1998, by 2002 this had increased to $14 \%$.

- The most significant gains were seen in primary industrial sub-sectors, which saw an increase of over $50 \%$ in terms of the contribution of electricity to total energy consumed.

These changes were fuelled by economic globalization which increased demand for manufactured, export driven products (Saran et al., 2005), as well as the reforms of stateowned enterprises active in primary resource areas of the economy (Wu et al., 2005). The shift towards manufacturing in the structure of China's industrial sector has led to a decrease in the energy intensity of the entire sector because manufacturing sub-sectors, in general, are less energy intensive than for primary sectors of industry. Nonetheless, electricity demand in China's industry sector is still dominated by economic activity in industrial sub-sectors active in the processing of raw primary products useable for manufacturing sub-sectors, such as the production of raw chemical materials and chemical products, smelting and pressing of ferrous and non-ferrous metals, and production of hot steam, water and electricity. 


\subsubsection{Decomposing the trends}

In order to quantify the impacts of changes in activity, structural and intensity on electricity demand, equations 7 through 17 are used in conjunction with the empirical data overviewed in table 10 (see table 12). The first column of the table presents the results of the additive decomposition analysis, while the latter presents the results from the multiplicative decomposition analysis.

Table 12. Factor contributions to changes in industrial sector electricity demand between 1998 and 2002

\begin{tabular}{|lr|r|}
\hline Activity & $\begin{array}{c}\text { Absolute } \\
\text { change }\end{array}$ & $\begin{array}{c}\text { Percentage } \\
\text { change } \\
\text { Structure }\end{array}$ \\
Intensity & -8 & $170.10 \%$ \\
Total & -242 & $99.40 \%$ \\
Residual & 329 & $82.71 \%$ \\
\hline
\end{tabular}

It is evident that the single most important factor that has shaped overall electricity consumption in China's industrial sector has been increases in industrial activity. The most important factor countering these increases in demand was decreases in the electricity intensity of electricity use per unit of economic activity. By 2002 electricity intensity per unit of industrial value added were only $83 \%$ of 1998 values.

Similar to the majority of conclusions obtained on industrial energy consumption, the least important of the factors investigated were changes in industrial structure. According to the decomposition analysis completed, this effect resulted in demand being approximately $99 \%$ of 1998 values in 2002 . Using the results from the additive decomposition, this shows that there was an approximate 8 TWh decrease in demand between 1998 and 2002 as attributed to structural shifts in China's industrial sector. This 
indicates that structural changes did not significantly contribute to lowering demand for electricity in China's electricity over this study period, and certainly was much less an influencing factor than the decreases seen for changes in electricity intensity per unit of industrial activity.

Since analysis of the 1998 to 2002 period was also completed at the two-digit sub sector industry level, the impacts of changes in both structure and intensity on electricity demand for these individual sub-sectors can also be ascertained. Figure 7 summarizes these changes by secondary - manufacturing, secondary - processing and primary sectors of industry (see Appendix C for details by sub sector):

Figure 7. Structural and intensity impacts on industrial sector electricity demand (1998 - 2002) (TWh)

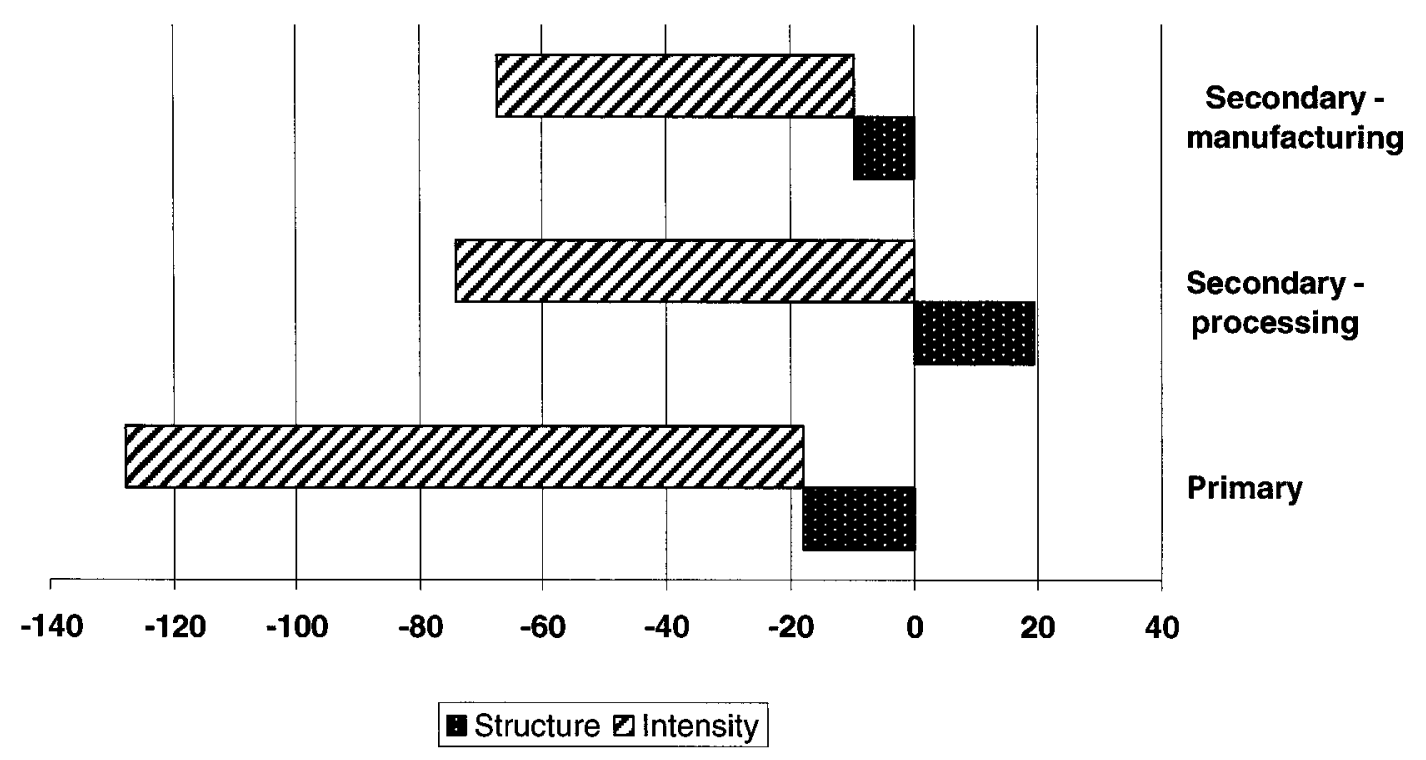

The individual sub-sectors that contributed to the majority of intensity change related decreases in electricity demand between 1998 and 2002 were related to primary industry: of the total $242 \mathrm{TWh}$ decrease in electricity demand due to changes in intensity, $110 \mathrm{TWh}$ 
(45\%) were associated with sub-sectors in primary industry. Although these sectors only contributed to $26 \%$ of the total value added of the entire industrial sector in 2002 , because heavy industry requires so much electricity per unit of value added, changes in these sectors have significant impacts upon total electricity demand. There were four sectors in particular which contributed significantly to the overall decrease in electricity intensity, all related to the processing of raw material for industrial purposes. Of these, the most important was the chemical material and chemical products sector, which would have contributed to an additional 42 TWh of demand if its electricity intensity had not decreased.

\subsubsection{Considering the additional effect of changing fuel shares}

The above analysis does not fully account for shifts in fuel shares between electricity and other forms of energy. This factor would mostly influence the obtained values for measures of the electricity intensity of industrial activity and value added. For example, if a fuel shift towards electricity away from another energy source were to occur, then this would appear in the analysis as an increase in electricity intensity per unit of economic activity. It would give a false representation of decreases in energy efficiency as it would be a fuel shift and not an actual change in efficiency. Based upon equations 16 and 17, table 13 presents the effects of fuel shifts in China's industrial sector on electricity demand when considering the period spanning from 1998 to 2002 . Again, the left hand column gives the outputs of the additive decomposition analysis, while the right gives the outputs of the multiplicative. 
Table 13. Factor contributions (including changing fuel shares) on industrial sector electricity demand

\begin{tabular}{|lr|r|}
\hline & $\begin{array}{c}\text { Absolute } \\
\text { change }\end{array}$ & $\begin{array}{c}\text { Percentage } \\
\text { change }\end{array}$ \\
Activity & 579 & $170.10 \%$ \\
Fuel shift & 245 & $121.18 \%$ \\
Intensity & -486 & $68.25 \%$ \\
Structure & -8 & $99.40 \%$ \\
Total & 329 & $139.84 \%$ \\
Residual & 0 & 0 \\
\hline
\end{tabular}

The analysis suggests that shifts towards electricity led to an increase of nearly $245 \mathrm{TWh}$ of electricity demand. This means that, based upon the rationale that fuel shifts would either hide or exaggerate changes in energy efficiency, energy efficiency likely decreased much more significantly than the earlier results had suggested. From the above, it appears that decreases in energy efficiency resulted in a decrease in electricity demand equal to nearly $490 \mathrm{TWh}$ of electricity.

Similar to the results obtained for the structural and intensity effects, the above analysis can be presented at the sectoral level for the 1998 to 2002 period (see figure 8 for sectoral summaries and Appendix $\mathrm{C}$ for details at the sub sector level): 
Figure 8. Impacts of changing fuel shares on industrial sector electricity demand (1998 - 2002) (TWh)

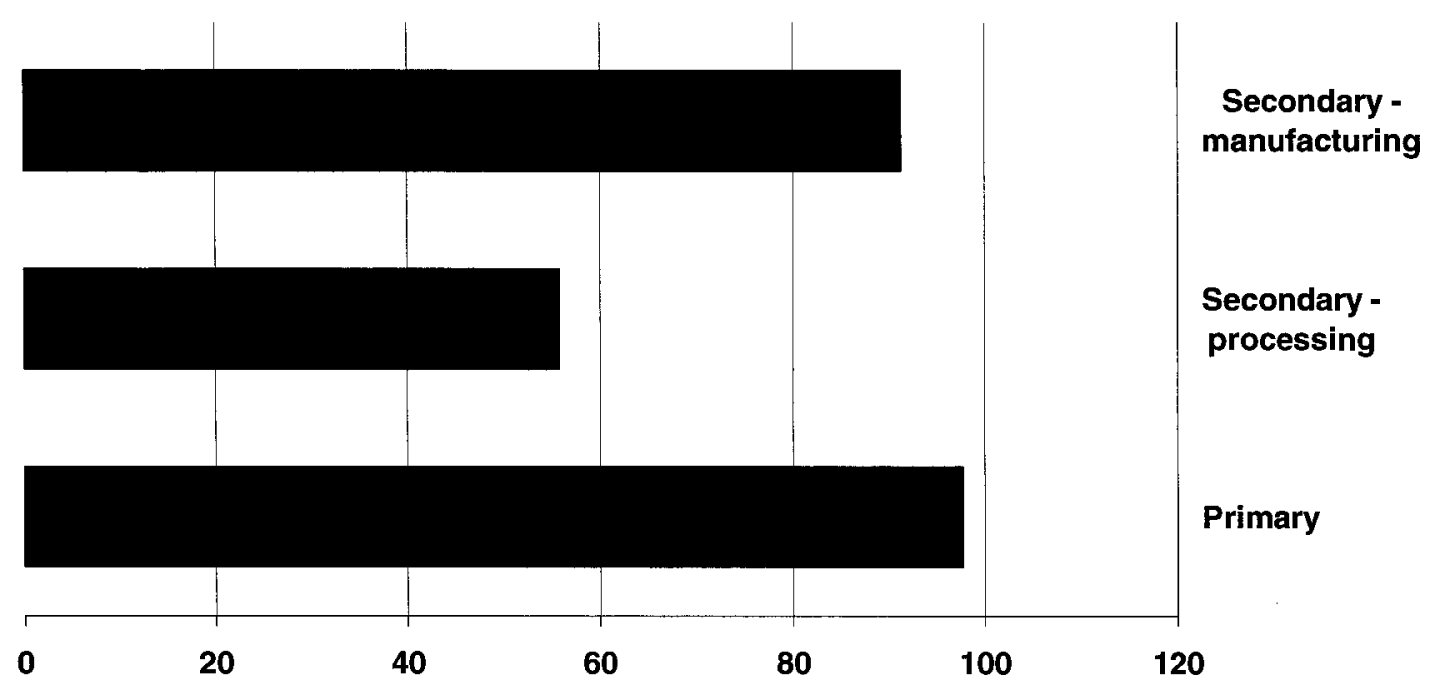

Across every sector, there were some level of fuel switches towards electricity between 1998 and 2002. The most significant of these occurred in primary sector, in particular the mineral refining and the production of electricity and steam sub-sectors.

The increases in the efficiency evident from the above analysis likely originated from a number of sources. First, it has been discussed that most of the efficiency gains took place in heavy industry. These industrial sub-sectors continue to be dominated by State-owned enterprises, a sector which has been the target of government reform in order to improve operational efficiencies. Any efficiency gains would also likely translate into an increase in the value added of product produced, resulting in a decrease in electricity intensity when measured by value added. In addition, the central government has also established many policies to help increase end use energy efficiency. One of the most prominent of these policies was the Energy Conservation 
Law passed by the State Council in 1997, right at the start of the period of analysis. This not only involved lower-level governments implementing 164 state standards for energysavings but also saw the establishment of the China Energy Conservation Investment Corporation, an entity meant to help implement and facilitate energy efficiency and conservation in the industrial sector. Of relevance to electricity demand, approximately 650GWh of demand has been targeted so to improve the electrical efficiency of pumps, fans and motors (McKane et al., 2005). This electrical equipment contributes upwards of $50 \%$ of electricity consumption in the industrial sector. Efficiency and conservation gains were also made through investments in heat and power cogeneration, district heating, waste heat and gas recovery and other major energy saving projects.

As was indicated earlier, using aggregate data to decompose energy demand can exaggerate intensity relative to structural effects. Thus, this tempers somewhat the strong evidence put forward for increases in efficiency contributing to the majority of the electricity intensity decrease in China's industrial sector, and suggests that although this most likely did contribute to the majority of change, structural effects may have had a marginally higher impact than shown in the analysis.

\subsubsection{Conclusion}

Factors that have influenced changes in electricity consumption in China's industrial sector have been focused upon as industrial activity in China contributes to over $70 \%$ of total electricity demand in the country. Therefore, changes in this sector have large implications on the energy sector in its entirety.

A number of key conclusions were made. It was found that rapid increases in industrial activity have had the most important implications on increasing electricity 
demand in the sector. These increases were countered by efficiency gains and structural changes in the economy. Concurrently, shifts in the types of fuel used led to an increase in electricity demand, which, in the 1998 to 2002 period, exceeded the decreases resulting from efficiency gains. The most important factor that has curtailed electricity demand has been the decreasing energy intensity of economic activity in the industrial sector.

The determination that it was efficiency gains, not structural effects, which led to the majority of change in the lowered electricity intensity that was observed in China's electrical sector between 1998 and 2002 is important on a number of fronts. First, it demonstrates that decomposition analysis can be used to help identify and quantify the contributions of different factors that are relevant to the demand of electricity, and therefore, relevant to energy and environmental policy targeting the electrical sector in its entirety. In the context of the CDM this is important as such analysis can help both illustrate and quantify what might be considered part of the baseline in terms of 'natural' economic progression. For example, a continuation of policies or economic processes leading to increases in energy efficiency would reduce the additionality of an energy efficiency project in the demand sector.

\subsection{Using decomposition in the electricity supply sector}

Since most CDM projects will be implemented into the supply side of the electrical sector, it is important to ask which factors have played a role in shaping the emission intensity of electricity provided to end users, what were the quantified contributions of these factors, and finally, to ask how these might change into the future.

Decomposition analysis is used to quantify factors leading to changes in the volume of $\mathrm{CO}_{2}$ emitted from electricity generation in China. For this the Laspeyres 
decomposition technique is used because it is arguably an easier and more accessible technique to use (a criteria for CDM baseline methods). The general decomposition approach used takes into account multiple factors that influence the carbon intensity of electricity delivered to the end user (measured by the volume of $\mathrm{CO}_{2}$ associated with each unit of electricity delivered to the end use $\left.\left(\mathrm{kg} \mathrm{CO}_{2} / \mathrm{kWh}\right)\right)$. These include changes in end use efficiency, changes in the mix of hydro, thermal, and nuclear to generate electricity (the generation effect), changes in fuel shares used to generate electricity (the fuel mix effect), the efficiency of electricity generation (the efficiency effect), the amount of electricity used during the process of electricity generation (the auxiliary effect) and electricity losses incurred during transmission and distribution (the T\&D effect). For this the Laspeyres decomposition technique is used because it is arguably an easier and more accessible technique to use by analysts undertaking such baseline studies than approaches based upon more complicated logarithmic methods.

A number of other studies have examined the progression of environmental impacts associated with the electrical sector using the decomposition technique. Shrestha and Timilsina (1997), for example, analyzed the electrical sector of 12 countries in Asia for the period spanning 1980 to 1990 . Here they found that the primary driver of increases in GHG emissions during this time were changes in the types of fossil fuels used to generate electricity. Shrestha and Timilsina (1997) found that the generations mix effect played the most significant role in China, Thailand and South Korea, while changes in fuel intensity were most important in the case of India, Japan and Taiwan. 


\subsubsection{Methods and data}

In order to identify the specific roles that changes in the generation mix, fuel mix, efficiency, etc. play in changing the emission intensity of electricity generated in China, the Laspeyres form of the decomposition technique is used. This is form is used because the results are easy to interpret, thereby meeting the criteria given by the UNFCCC of using transparent approaches for baseline formation. The analysis completed draws upon an identity established by Nag and Parikh (2005) who used it to study factors contributing to the emission intensity of electricity in India. This identity focuses on decomposing the emission intensity of electricity consumed by the end user, and is written as follows:

$$
\frac{E}{P C}=\frac{E}{T G} \times \frac{T G}{T O T G}\left(=S_{i}\right) \times \frac{T O T G}{N e t G}\left(=A U X_{i_{i}}\right) \times \frac{A v l t y}{P C}\left(=T D_{i}\right)
$$

Where:

$\mathrm{E}=$ total emissions

$\mathrm{PC}=$ total power consumed

$\mathrm{TG}=$ total thermal generation

TOTG $=$ total power generation

NetG $=$ net generation, or TOTG minus auxiliary consumption, and, Avlty $=$ power consumption minus transmission and distribution $(T \& D)$ losses. 
The three remaining terms are the values calculated from each of the above relationships:

$S_{i}=$ the percentage of thermal electricity to total electricity generated in year $i$, $\mathrm{AUX}_{\mathrm{i}}=$ the percentage of net generation to total generation in year $\mathrm{i}$, and $\mathrm{TD}_{\mathrm{i}}=$ the percentage of electricity to total electricity consumption after deducting $\mathrm{T} \& \mathrm{D}$ losses in year $\mathrm{i}$.

These terms are used directly inside the decomposition formulae derived, as discussed.

The first term on the right side of the above identity (E/TG) can be further decomposed into the emission effects resulting from changes in the fossil fuel mix and changes in the energy intensity of electricity generated, as given:

$$
\frac{E}{T G}=\sum_{I} \frac{e c_{i} \times f_{i}}{\sum f_{i}}\left(=F_{i}\right) \times \frac{\sum f_{i}}{T G_{I}}\left(=I_{i}\right)
$$

Where:

$\mathrm{ec}=$ emission coefficient of fuel type $\mathrm{i}$ given in tonnes of carbon per gigajoule of energy (TC/GJ), and $\mathrm{f}=$ fuel type (again in GJ). 
The results of equation 19 give the emission intensity of fuel consumption in year $\mathrm{i}\left(\mathrm{F}_{\mathrm{i}}\right)$. If $f$ for year $i$ (i.e. $f_{i}$ ) in the last term above is converted into the same energy units as used for the ones measuring thermal generation (TWh), then this will give energy intensity as a ratio between energy used and energy available for consumption in year $i\left(I_{i}\right)$. This calculated parameter is used in later analysis.

Based on the above discussion, the Laspeyres index is modified so that it can account for the factors investigated. This includes:

For generation mix effect:

$$
G M_{\text {effect }}=\sum_{i} S_{t} \times I_{o} \times F_{o} \times T D_{o} \times A U X_{o} / S_{o} \times I_{o} \times F_{o} \times T D_{o} \times A U X_{o}
$$

Where:

$\mathrm{GM}_{\mathrm{effect}}=$ generation mix effect,

$S=$ structure effect,

$\mathrm{I}=$ energy intensity effect,

$F=$ fossil fuel mix effect,

$\mathrm{TD}=\mathrm{T} \& \mathrm{D}$ effect, $\mathrm{AUX}=$ the auxiliary effect.

The subscripts ${ }_{o}$ and ${ }_{t}$ represent the initial and subsequent years in the times series decomposed, while ${ }_{\mathrm{i}}$ is a running index over the years. 
For fossil fuel effect:

$$
F_{\text {effect }}=\sum_{i} S_{o} \times I_{o} \times F_{t} \times T D_{o} \times A U X{ }_{o} / S_{o} \times I_{o} \times F_{o} \times T D_{o} \times A U X{ }_{o}
$$

For fuel intensity effect:

$$
F I_{\text {effect }}=\sum_{i} S_{o} \times I_{t} \times F_{o} \times T D_{o} \times A U X_{o} / S_{o} \times I_{o} \times F_{o} \times T D_{o} \times A U X_{o}
$$

For the auxiliary effect:

$$
A U X_{e f f e c t}=\sum_{i} S_{o} \times I_{o} \times F_{o} \times T D_{o} \times A U X_{t} / S_{o} \times I_{o} \times F_{o} \times T D_{o} \times A U X_{o}
$$

For the T\&D effect:

$$
T \& D_{\text {effect }}=\sum_{i} S_{o} \times I_{o} \times F_{o} \times T D_{t} \times A U X_{o} / S_{o} \times I_{o} \times F_{o} \times T D_{o} \times A U X_{o}
$$

\section{Data}

In order to use the above formulae, information on for each of the associated factors had to be located. This data is summarized below.

For understanding historical electricity demand and the factors influencing this (i.e. changes in GDP and electricity intensity of GDP), the China Energy Databook made available by the Lawrence Berkeley Research Laboratory (LBRL, 2004) for the purposes of this research provides details on changes in these parameters for the years between 1980 and 2002. This information originated from various years of China's Statistical 
Yearbook as published by the China Statistical Press (CSP, 1998-2004). This data source also included data on energy efficiency of thermal generators, fuel shares by type of fuel (coal, coal products, natural gas, fuel oil, LPG), auxiliary use of electricity, T\&D losses by year, and generation mix (hydro, thermal and nuclear). Data on the carbon equivalent emission intensity of primary fuels used in electricity generation were based on the international defaults contained within the Intergovernmental Panel on Climate Change methodologies for baseline formulation (IPCC, 1996).

\subsubsection{Results}

The emission intensity of electricity in China has progressively been decreasing with time. In 1980, the generation of electricity in China resulted in the release to the atmosphere of nearly $1.25 \mathrm{kgCO} 2 \mathrm{e}$ per $\mathrm{KWh}$ of electricity delivered to the end user. By 2002 , this had decreased by nearly $24 \%$ to approximately $0.95 \mathrm{kgCO} 2 \mathrm{e}$ per $\mathrm{KWh}$. This decrease however was not uniform across time (see figure 9): 
Figure 9. Decomposition of the carbon emission intensity of electricity generation

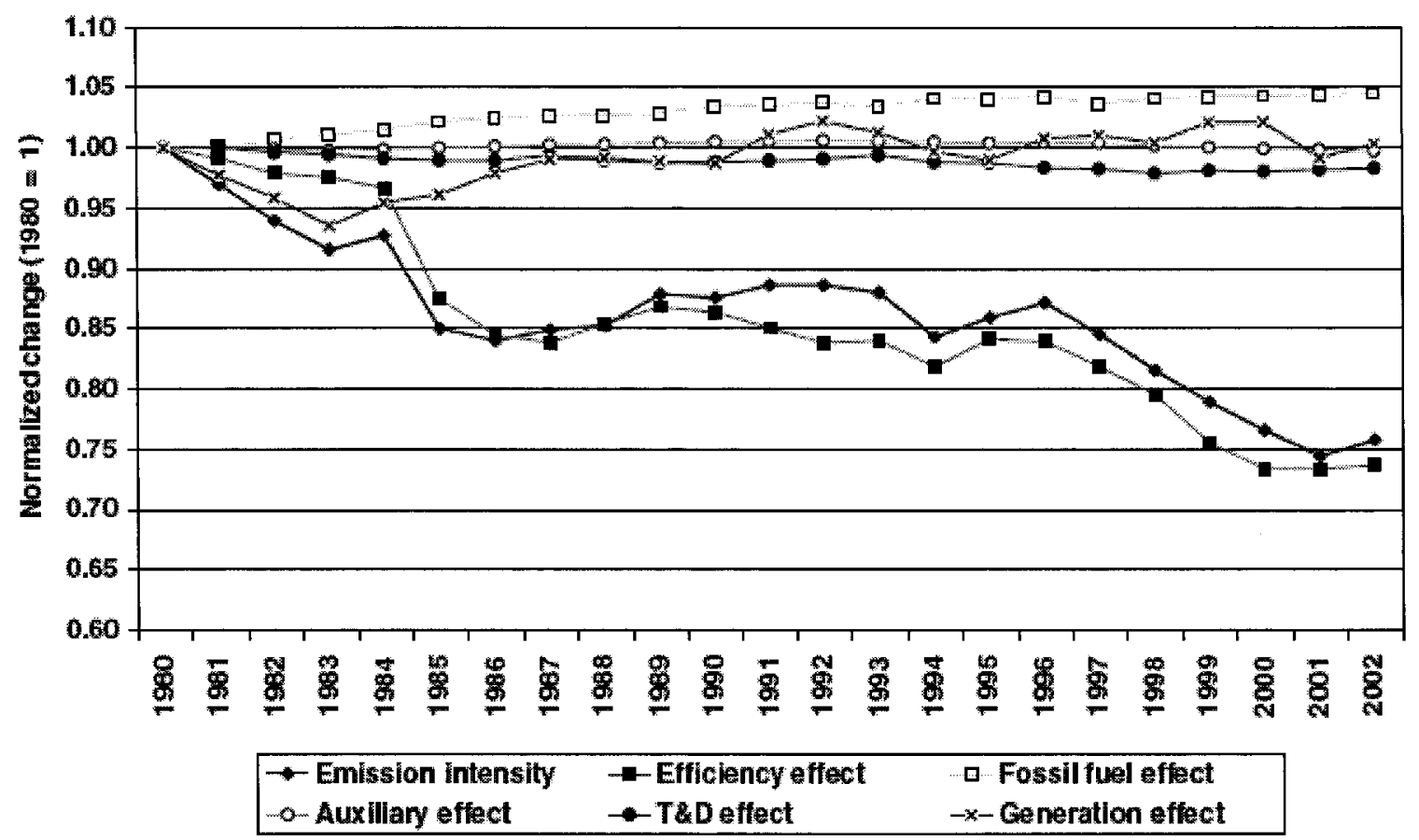

A number of key points are noted concerning the chronological series of events related to the trends in the emission baseline:

- Increases in efficiency and more hydro-generated electricity led to a declining baseline between 1980 and 1985, but over the next five years policy emphasized the construction of new capacity, thereby pushing the baseline higher;

- Throughout the early 1990's efficiency remained relatively constant, and with only minor changes occurring in source generation or fuel mix, the emission baseline remained relatively stable;

- In the mid 1990's China's central government established a new general policy to shut down small capacity, coal-based generators in order to improve system efficiency, leading to a large increase in efficiency during this time. 
- Generally, the fossil fuel mix, T\&D, and auxiliary effects played only a small part in contributing to overall changes in the baseline.

Seeing its important contribution to the above, the trends in the efficiency of electricity generation are examined closer for the period spanning from 1980 to 2002 (see figure 10):

Figure 10. Trends in the energy efficiency of China's electrical sector

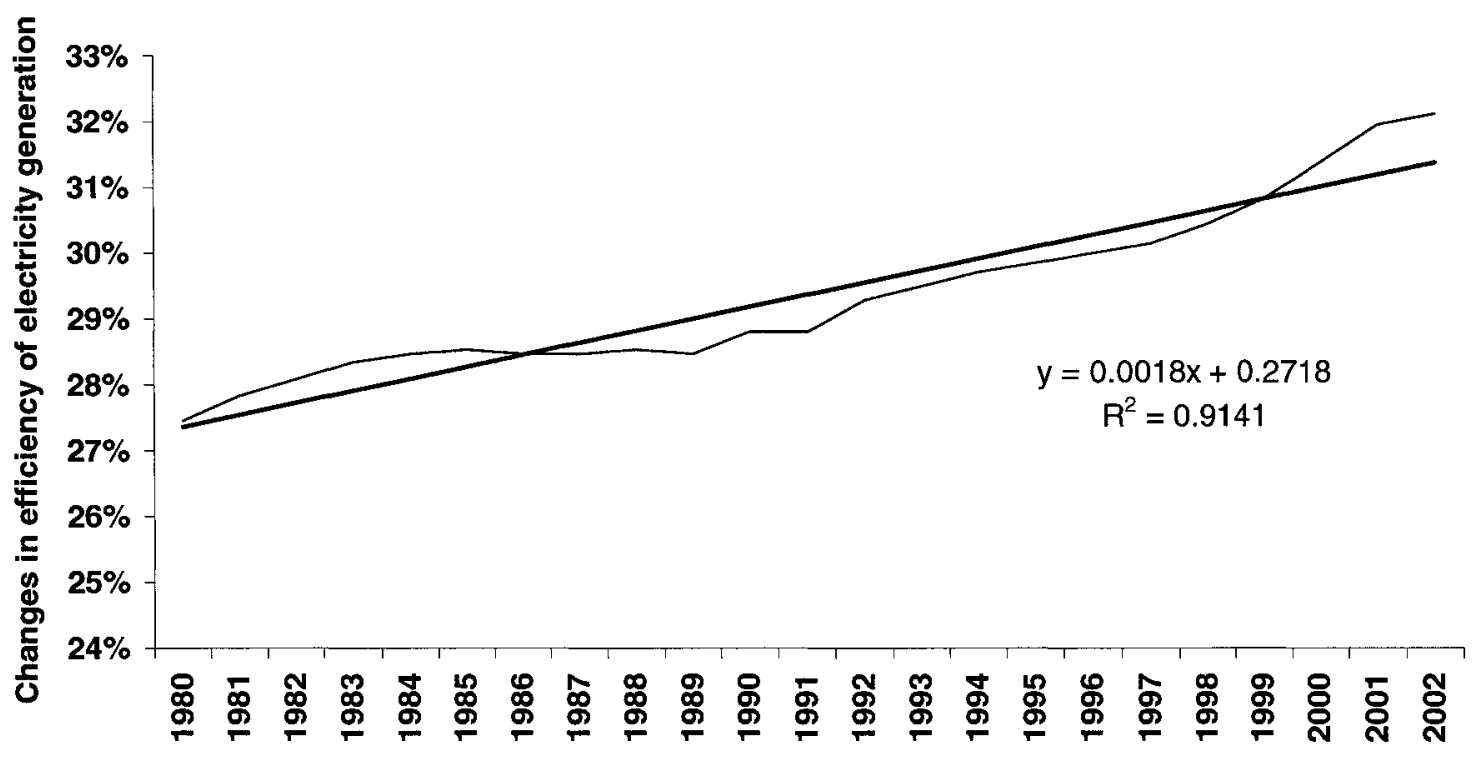

The $\mathrm{R}^{2}$ value of $>0.9$ underscores the consistency and strength of this trend increase over the entire period. Overall, the annual increase in the efficiency of thermally generated electricity approached $0.7 \%$.

This discussion helps to emphasize that increases in energy efficiency have had the most significant impact on decreasing the emission intensity of electricity delivered to the end user in China. Conversely, largely due to increases in the contribution of coal, changes in the fossil fuel mix actually increased the emission intensity of electricity over this time. Meanwhile, the T\&D and auxiliary effects play only a small part in 
contributing to overall changes in the emission intensity of electricity delivered to the end user. This is because, even though these terms experienced significant improvements over the period studied, their influence is much smaller due to their smaller contribution to total electricity generated.

\subsubsection{Conclusions}

In this section, using the Laspeyres decomposition technique, historical data has been analyzed to understand changes in emissions from electricity generated in China. This analysis forms the basis for the operationalization of this baseline method through the application of scenarios. The steps completed in this analysis showed that gains in the efficiency of thermal generators has been the most important factor leading to decreases in the emission intensity of electricity generated in China. Since the analysis was focused on changes in the emission intensity of electricity generation rather than actual changes in emissions, changes in economic activity were not explicitly considered in the decomposition equations formulated.

A caveat with this approach, however, is that using decomposition analysis within a country experiencing such robust economic growth may not be able to adequately consider some economic uncertainties unless these are explicitly considered in scenarios generated. In China, these include such possibilities as resource constraints emerging in the primary fuels sector or the level of financial resources available or allocated towards capital investment and new technology 


\subsection{Chapter conclusions}

The chapter has presented decomposition analysis of both electricity demand and supply. As suggested in the introduction, this two-pronged analytical effort is relevant and important on a number of fronts. First, the electrical sector acts as a demand-supply system, interrelated and interconnected - efforts and events in the demand sector can have important consequences on the supply sector. It should be expected that there would be many CDM, climate change mitigation projects, and associated energy security efforts in the future in terms of both demand and supply.

The substantive results of the analysis lead to several conclusions. In the demand side of China's electrical sector, particularly in the country's important industrial base, government-led efficiency gains have been the most important factor that have contributed to decreases in the electricity intensity of electricity demand in recent years. Structural changes were also important, both originating from globalization processes and central government programs designed to increase China's manufacturing base, but the majority of change was related to efficiency gains. The analysis suggests that the 'business as usual' scenario will be increasing efficiency gains into the future, whether that is from technological change or operational practices. It is important to note that in most recent years China's central government has invested fewer financial resources per unit of industrial output into either efficiency or conservation, leading in part to a rise in the energy intensity of China's economy. It was also shown that changes in efficiency was one of the most important factors that led to changes in the emission intensity of electricity generated, which in turn suggests continued increases in efficiency should be part of a BAU scenario. 
The analysis and the conclusions reached have helped to demonstrate the strengths of the decomposition technique for analyzing and understanding both electricity demand and supply. The technique is powerful when trying to assess the impacts of the macroeconomic processes in the past, and establishing a BAU scenario. However, to best assess this technique for understanding the future, which is crucial for baseline studies, a much more detailed examination must be undertaken of how the electrical sector might progress into the future. This, alongside the generation of a baseline using the simulation technique, will then allow us to better assuage the strengths and weaknesses of these different baseline methods. 


\section{China's electrical sector into the future}

Synopsis: In this chapter, a conceptual framework that can be used to help understand the future development of China's electrical sector is developed. This framework conceptualizes the electrical sector as a demand-supply system with domestic and global inter-linkages. Since a baseline must properly account for both the economic and policy landscape that a CDM project might be developed within, the framework specifically builds upon key technological, socio-economic drivers internal within China and those acting upon it, with these in turn related to current policy setting within the country. These include factors affecting electricity demand (e.g., economic growth, structure, energy efficiency, urbanization, and per capita income growth) and supply (e.g., deregulation, initiatives to promote natural gas, nuclear and renewable energy, air pollution regulations, price developments for coal and natural gas, and changes in generation technology). The building of this framework lays the groundwork for a scenario exercise on the greenhouse gas and fuel consumption impacts of different developmental paths for China's electrical sector. The general storylines of these scenarios are presented at the end of this current chapter, and analyzed in the next chapter.

\subsection{Chapter introduction}

The future GHG emission impacts of China's electrical sector are studied. In order to do this, first, a conceptual framework is designed that can be used to help understand how GHGs from China's generation of electricity could progress into the future. This framework is designed partially upon a review and assessment of a) historical drivers 
influencing the electrical sector b) how these might develop into the future, and c) policy interventions associated with these drivers. The historical and future drivers include those affecting electricity demand (including economic growth, economic structure, energy efficiency, urbanization and per capita income), and electricity supply (including central government initiatives to promote natural gas, increase the role of renewables and nuclear power, decrease local pollution, and the interplay with international energy markets). The final part of the chapter then uses the conceptual framework as a basic to envision three different scenarios to the year 2020 .

\subsection{Drivers affecting electricity demand}

Recent changes occurring in China offer a foreshadowing of possible future developments in China's economic structure, urbanization, and the consumption characteristics of the population. These factors will drive the rate and direction of the country's electricity demand.

Structural changes towards more energy and electricity intensive industrial subsectors are occurring and likely will continue to occur due to an upgrading of the household consumption structure, continued urbanization and resultant infrastructure construction. The urban to rural population ratio is still comparatively low on an international basis, even after years of rapid urbanization. This suggests urbanization will continue for many years and contribute to an increase in electricity demand due to the higher energy intensity of urbanites. Furthermore, per capita incomes are rising and will compound with other drivers increasing electricity demand in the household sector.

A number of broad-based economic, technological and social processes underlie these drivers, which in turn are impacted by policy decisions made by China's central 
government. The most significant of these include: processes and policies related to the viability of continued rapid economic growth (including the presence of inflationary pressures throughout the economy and financial stability related to non-performing loans); industrial sector restructuring and the energy efficiency of electricity end-use; and, on the social level, the equity of the increasing wealth of the Chinese people and that personal consumption will increase at the expense of personal savings.

\subsubsection{Future economic growth and change}

Fundamental to electricity demand in all economic sectors is the strength and structure of economic growth. China's central government has made the quadrupling of GDP by 2020 a national goal, stating that it wants to "build a well-off society in an all-round way." (Shixian et al., 2005). By this time, China's per capita GDP could surpass USD $\$ 10,000$ when calculated on the basis of purchasing power parity (PPP). ${ }^{8}$ A number of major uncertainties underlie these economic changes however, as described.

\section{Overheating in the economy}

In order to sustain a continuation of economic growth and not risk 'overheating' of its economy, China's government must ensure that the demand for goods, commodities, and services does not outstrip supply, nor that surplus and unproductive investment occurs into economic sectors such as real estate or heavy industry. If any of these were to occur, excessive inflation could result, forcing potentially rapid increases in lending rates which would in turn slow down real economic growth. Indicators of possible economic strains

\footnotetext{
${ }^{8} \mathrm{PPP}$ is a method of measuring the relative purchasing power of different countries' currencies over the same types of goods and services. Different goods and services are priced differently in different countries, and depending on the strength of the relative currencies, a dollar in one country might be able to buy more than in another country.
} 
began to emerge throughout 2003 and 2004 when the core inflation rate rose to nearly 3.9\%. China's central bank responded by initiating a round of monetary tightening and urging many of the state-controlled banks to reign in their loans to industry. The reduction in credit slowed down investment and lowered inflation rates moderately.

\section{Non-performing loans and reform of the banking system}

Numerous authors have emphasized that the historical lending practices of state-owned banks and the wealth of non-performing loans to SOEs present significant risks to China's banking system (Ogilvy, 2000; Chang, 2001). Estimates place these loans as high as $25 \%$ of total loans outstanding. This creates significant risk because the banking system is the primary route for financing in China - equity and bond markets are insignificant compared to their role in more advanced economies. Sustainable economic growth depends on a banking system being able to efficiently and productively allocate financial resources. The non-performing loans situation reflects that the banking sector in China is in drastic need of reform and that lending decisions are often made by political bargaining, not on the financials or credit risk of the loan. For China to achieve the economic goals it has established, more drastic reforms of its banking sector must take place.

\subsubsection{Changes in the industrial sector}

\section{Industrial restructuring}

Throughout the 1980's and 1990's, China's economy was strongly influenced by in-flows of capital investment into the manufacturing sub-sectors of its economy, much of which originated from investors in Hong Kong seeking to take advantage of China's low cost 
labor market. State-owned and privately held companies also sought out export markets. It was these processes that led to much of the industrial sector restructuring discussed in previous sections. China's population is now growing wealthier, infrastructure is being built on a very large scale, consumption is rising, and this is resulting in increased output from heavier industrial sub-sectors such as chemicals, building materials, and the production of chemicals, metals and steel. As these industrial sub-sectors are more energy and electricity intensive, this is causing electricity demand to accelerate.

Such a process of restructuring to more energy-intensive sectors should be expected as China continues to develop. In the past, much of its economic growth was achieved by foreign investment into the country's manufacturing sectors, and alongside significant improvements in operational and technical efficiency, electricity intensities declined. Into the future however energy and electricity intensity will likely be driven higher due to a more consumption-driven economic growth.

\section{Energy efficiency in the industrial sector}

If the industrial sector continues to restructure towards more electricity intensive subsectors as expected, the central government will either need to heighten programs to increase energy efficiency or speed up the construction of electricity generating capacity. If not, the economy could face electricity shortages such as what occurred throughout 2003 and 2004. With restructuring, demand could accelerate beyond the capacity additions planned, causing shortages to occur. Energy-efficiency programs are underway focusing upon electricity end-use, including efforts to improve motors, pumps and fans (McKane et al., 2005), and the retrofitting of buildings, but since 2000 China's central 
government has invested less into energy efficiency per total energy investment that it did in the past (Sinton et al., 2005).

\subsubsection{An urbanizing, wealthier, but economically fragmented society}

\section{An urbanizing population}

Urbanization is likely to continue in China and a number of important factors will contribute. Tight regulations historically used to control rural to urban movements have been lowered and it is easier for a rural person to move into an urban area in search of higher paid employment (Zhang et al., 2003). This will likely coincide with increasing labor productivity in China's agricultural sector, currently only a 50th of that in the U.S, decreasing employment prospects in rural areas, and pushing these workers to urban regions (Johnson, 2001). Overall, continued urbanization will increase overall energy demand and intensity.

Shifting economic growth towards consumption

China's central government has indicated that it desires a greater proportion of economic growth to be consumer-based. This would follow models of development in more established and mature economies, and suggests that per capita income will continue to rise. Increased consumption will likely come in the form of an increased ownership of home appliances and other energy consuming good items, furthering energy and electricity demand. This could also mean significantly higher electricity demand during peak hours.

Growing income inequities and social unrest 
Incomes may be rising, but there are large inequities between residents in the east and those in the west, and those in the city versus those in the country. This has the potential to fragment the country's social fabric, a dilemma for a country which wants to develop its economy rapidly as social cohesion is a requirement for building and retaining human capital. In order to reverse the growing income inequities occurring throughout China and the potential for social unrest, the central government has indicated that it will set goals to equalize incomes across urban- rural and east-west divides (Beech et al., 2006).

\subsubsection{Decision nodes}

It is important to consider the policy dimensions of the above drivers with regard to their effect on electricity demand. Four 'decision nodes' where domestic policy will have significant impact have been identified, including those affecting: macroeconomic level growth and stability; industrial structure; end-use energy efficiency; and, policies affecting consumer demand (see table 14): 
Table 14. Decision nodes affecting electricity demand in China

\begin{tabular}{|c|c|c|}
\hline Driver & $\begin{array}{c}\text { Relevance for electricity } \\
\text { demand }\end{array}$ & Associated domestic policies \\
\hline $\begin{array}{l}\text { Economic } \\
\text { growth and } \\
\text { equity of } \\
\text { distribution }\end{array}$ & $\begin{array}{l}\text { Rate and stability of } \\
\text { electricity demand }\end{array}$ & $\begin{array}{ll}\text { - } & \text { Monetary policy } \\
\text { - } & \text { Banking reforms } \\
\text { - } & \text { Lending practices } \\
\text { - } & \text { Rural and west development } \\
& \text { policy }\end{array}$ \\
\hline $\begin{array}{l}\text { - Industrial } \\
\text { structure }\end{array}$ & $\begin{array}{l}\text { - Changing electricity } \\
\text { intensity of industry }\end{array}$ & - Lending practices \\
\hline $\begin{array}{l}\text { Industrial } \\
\text { end-use } \\
\text { efficiency }\end{array}$ & - Demand reduction & $\begin{array}{l}\text { - Targeted energy-efficiency } \\
\text { programs } \\
\text { - Policies guiding technology } \\
\text { diffusion }\end{array}$ \\
\hline $\begin{array}{l}\text { - Consumer } \\
\text { demand }\end{array}$ & $\begin{array}{l}\text { Effects on industrial } \\
\text { restructuring } \\
\text { towards electricity } \\
\text { intensive sectors } \\
\text { - Electrical appliance } \\
\text { ownership } \\
\end{array}$ & $\begin{array}{l}\text { - } \\
\text { - Tax and incentive programs } \\
\text { - Targeted energy efficiency } \\
\text { programs }\end{array}$ \\
\hline
\end{tabular}

\subsection{Electricity supply}

The structure of electricity supply is largely a function of the relative price of primary energy sources, technologies available for generation, and environmental regulations.

Central initiatives are important to consider as these can affect any of these above factors or impact on GHG emissions and fuel consumption in other ways.

\subsubsection{Domestic policy initiatives: painting a backdrop for decision- making}

Advancing natural gas

The National Development and Reform Commission (NDRC) have a long-term goal to increase natural gas demand to a range of 180 to 200 billion cubic metres (BCM) per year 
or $10 \%$ of total primary energy supply (TPES) by 2020 (IEA, 2002a). This represents a rapid rise from the $38 \mathrm{BCM}$ (two percent of TPES) consumed in 2003 (LBRL, 2004). China is emphasizing the use of natural gas in the electrical sector because it produces negligible amounts of sulphur dioxide $\left(\mathrm{SO}_{2}\right)$, although a side benefit is that natural gas produces only about half the amount of $\mathrm{CO}_{2}$ as does coal. Two key elements for meeting these established goals will be to secure adequate amounts of natural gas (discussed in detail in later sections), and to secure adequate financing. Building a natural gas infrastructure is very capital intensive and requires various routes for financing. However, if China's economy were to take a downturn trend, financial resources could be limited and reduce prospects for increasing the importance of natural gas as an energy source.

\section{Continuing the nuclear energy program}

While many countries are decreasing their nuclear energy programs, China is rapidly increasing its own (WNA, 2005). By the start of 2006, China had 11 nuclear power plants in operation with a total installed capacity equal to $8,350 \mathrm{MW}$, a significant rise from the $2,100 \mathrm{MW}$ of installed capacity in 2002 . Over the next 15 years, China plans to build as many as 32 large $1,000 \mathrm{MW}$ nuclear power reactors, bringing its total installed capacity to near $20 \mathrm{GW}$ by 2020 . However, similar to the building of a natural gas infrastructure, nuclear energy is very capital intensive and therefore the ongoing continuation of China's nuclear program will require a strong economy and adequate financial resources. 
Renewable energy law

On January 1st, 2006, China's 'Renewable Energy Law' (REL) entered into force (PRC 2005) (PRC, 2006). This law stipulates that renewables must contribute $10 \%$ of the national energy supply by 2020 , a significant increase from the current $2 \%$. Electricity grid operators will be obligated to purchase electricity generated from renewable sources (defined as all non fossil-fuel forms of electricity generation). This law will be facilitated through the use of low interest loans, tax incentives, and a cost sharing structure with other electricity generators enacted if marginal costs are higher than for non-renewables. It will be administrated by the NDRC, but implemented by governments at the regional and local levels. Decisions on regional targets will be based upon regional circumstances, including the availability of renewable energy, meaning that the success of this program will be determined by participation and decision-making at each level of the governmental hierarchy.

\subsubsection{Fuel costs and the interplay with international fuel markets}

Since fuel costs contribute to a significant proportion of the total costs of fossil-fueled electricity generation, expectations of future changes in fuel price affect the structure of electricity supply, especially when considering the comparative prices of coal and natural gas. In general, balances between demand and supply control the price structure of these fuel resources. Historically China could meet most of its energy resource requirements domestically, but now accelerating electricity demand has meant that energy resources must be imported from other countries. Thus, the growth of China's electrical sector is having ramifications globally and is influencing the price of these energy commodities 
internationally. The interlinkages of these components will become even more important as China's electricity demand continues to grow, as described in detail below.

\subsubsection{Coal}

Coal is expected to continue to be a major contributor to electricity generated into the future as China has large coal reserves, estimated by British Petroleum at 114 billion tonnes, or $12 \%$ of the world's total (British Petroleum, 2004). The majority of China's coal reserves are located in the country's north, and consequently, an extensive railway network has been developed to help transport coal from production to the rapidly growing eastern provinces. The related transportation costs mean that eastern provinces pay about $50 \%$ more for coal than regions in the coal rich north.

The supply of coal from domestic production has recently been negatively impacted by a restructuring of the coal sector in order to improve mine safety (Qingyi, 2004). This involved closing small mines and increasing output from large mines, which inadvertently caused the rate of coal production to slow, putting pressure on the government to reduce domestic exports to international markets. In the future, China will likely rely to some extent on imported coal, with source regions including Australia and Indonesia in the southeastern coastal provinces, and Russia in the north.

Due to continued rapid economic expansion and uncertain domestic production, there is a range of projections for China's coal supply-demand balance. Youguo (2004) estimates that by 2010 there will be an annual shortfall of $250 \mathrm{MT}$ of coal, and by 2020 , there will be a shortfall of 620 to $770 \mathrm{MT}$. Under this scenario, imports would equal nearly $30 \%$ of the total coal consumed, increasing China's dependence on import markets and changing the structure of international coal trade. Alternatively, the World Energy 
Outlook (IEA, 2005) suggests that the production of hard coal in China will increase and that the country will export 130MT of coal per year by 2030 .

\section{Coal imports and price}

As China becomes intertwined with the international coal market, it will become increasingly susceptible to international price movements. The U.S. Energy Information Agency estimates that by 2010 the nominal price of thermal coal available on world markets will be $5 \%$ higher than 2005 (EIA, 2005). This could rise by $50 \%$ by 2020 , and $72 \%$ higher by 2030 . These price estimates assume increased production and labor costs at coal mines globally, increasing international demand for coal due to continued global economic growth (particularly in China and India), increased prices for alternative energy sources, and that freight rates will increase. These same factors will also contribute to prices paid for imports into China, suggesting that prices will continue to strengthen into the future in this market as well.

\subsubsection{Natural gas}

Since natural gas has not been accessible in the past, it contributes less than $2 \%$ of all electricity presently generated in China. Now the central government has ambitious goals to raise natural gas use due to the fuel's environmental and operational benefits (IEA, 2002a).

\section{Supply and demand}

A fundamental challenge facing China's government is that planned increases in natural gas demand will exceed known domestic resources. The NDRC estimate that natural gas demand will approach $100 \mathrm{BCM}$ in 2010, and $200 \mathrm{BCM}$ in 2020 - an average annual 
increase of $10 \mathrm{BCM}$ from the $38 \mathrm{BCM}$ of demand in 2003 (IEA, 2002a). However, proven reserves have been estimated at 2 trillion cubic metres (TCM), and recoverable reserves of 1 TCM (Yamaguchi et al., 2004). Such reserve levels could not sustain the production needed to meet the ambitious demand targets set by the NDRC, and much of this demand will therefore have to be met by imports.

The potential size of import requirements can be appreciated by considering estimates of demand available from Petro China (i.e. CNPC), British Petroleum, and the NDRC themselves (IEA, 2002a; BP, 2004; Yamaguchi et al., 2004) in the context of potential domestic production given by the NDRC (see figure 11):

Figure 11. Projected demand and supply of natural gas in China

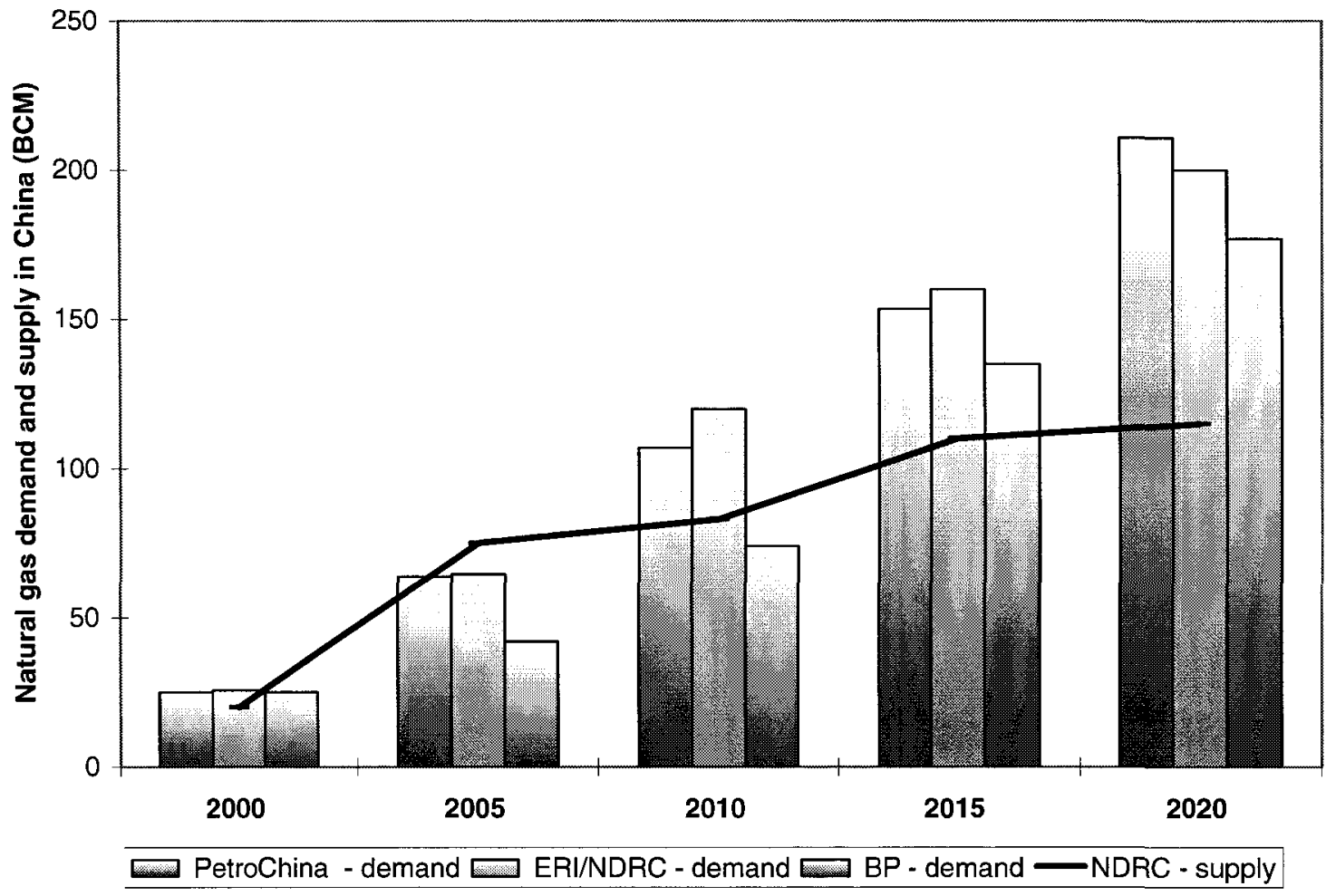


China's natural gas production base is not large enough to support a rapid expansion of its gas sector as hoped for by the central government and by 2020 China may have to import nearly $40 \%$ of its natural gas requirements.

\section{Natural gas imports}

There are two different possible sources for natural gas imports: constructing an extensive liquid natural gas (LNG) terminal system to receive international shipments of LNG by vessel, and long distance international gas pipelines to receive natural gas from other regions in central Asia and Russia. Currently, LNG terminals are being built in the eastern and southeastern provinces (including Shanghai, Guangdong, and Fujian) to receive LNG from Australia, Indonesia and the Middle East (Paik, 2004). International pipelines are still in discussion, but possibilities include a $30 \mathrm{BCM}$ capacity pipeline through northeastern China to North and South Korea from natural gas fields in Western Siberia, or a pipeline supplying gas to the west-east pipeline from locations in central Asia (Paik 2004). Each option has various risks associated with it, including acquiring the necessary financing, retaining adequate technical capacity to build the infrastructure, and ensuring the political stability of both source regions for the needed fuels and of North and South Korea.

The cross development of both LNG and international pipelines will result in competition between LNG and pipeline gas, although different mechanisms influence the final prices of each. Whereas the price of pipeline gas is largely determined by the sale price of the producer and the added costs of transportation and distribution, the global nature of LNG shipments and the ability to import from farther away regions mean that LNG prices are influenced by developments in a larger market. Before 2000, LNG prices 
for countries throughout the Asia-Pacific region were linked to the price of crude oil imports into Japan (known as the JCC (Japan Crude Cocktail)). In subsequent years, China emerged as a sizeable purchaser of LNG, establishing contracts with Indonesia and Australia lower than its East Asian neighbours, driving the average Asian price downwards and away from the JCC price formula (Yamaguchi et al., 2004). However, in most recent years, prices for both LNG and pipeline gas have risen, with LNG prices spiking globally due to accelerating global demand, supply problems in the North America market associated with hurricanes in the Gulf of Mexico, and rising capital costs (Seng-Kee, 2006). This has led to suggestions that China, who was able to secure longterm supply contracts at low prices in 2002 , in the future will not be able to attract the suppliers it needs unless it is willing to pay much higher market prices (Law, 2006). This could put into jeopardy the LNG terminal construction plans of the three companies active in developing LNG capacity (including the China National Offshore Oil Corporation, Sinopec and PetroChina). However, even if these prices temper over the next few years, due to increasing production costs and accelerating global demand for natural gas in general, most institutions providing long-term price estimates agree that the price of natural gas will rise faster than that for coal after 2010 (EIA, 2005; IEA, 2005).

These above parameters underlie the price assumptions used for the scenarios created in the chapter that follows, with the price of natural gas assumed to remain relatively stable in the short term (i.e. pre-2010), and then rising in the subsequent years faster than the price of coal. 


\subsubsection{Pursuing technological change}

The technology available for electricity generation is also a major determinant in GHG emissions. Historically, the most important processes affecting changes in generation technology in China were central policies to shut down small, inefficient plants, but into the future higher fuel costs and tighter emission limits on air emissions will mean that the implementation of more efficient and less polluting technology will become more important.

The main generation technologies that could play a significant role in China's electricity supply sector are described in brief below.

Clean coal technology

Due to the heavy reliance on coal for electricity generation, clean coal technology (CCT) will play an important role in changing the emission and fuel consumption characteristics of China's electrical sector. CCT is a technical system composed of new technologies (including technologies used in coal mining, combustion, conversion and pollution control) that can improve the efficiency of coal utilization and decrease environmental pollution (Zhufeng et al., 2001). However, compared to the sub-critical generation units currently in wide-spread use, the higher capital and operating costs and a lack of domestic capacity have been limiting factors to more broad based implementation of CCT.

Table 15 compares the characteristics of CCT with the sub-critical generation units currently in use. 
Table 15. Characteristics of sub-critical generation units versus clean coal technology

\begin{tabular}{|l|r|r|}
\hline \multicolumn{1}{|c|}{ Parameter } & $\begin{array}{c}\text { Sub-critical coal-fired } \\
\text { power projects }\end{array}$ & \multicolumn{1}{c|}{$\begin{array}{c}\text { Clean coal technology } \\
\text { power projects }\end{array}$} \\
\hline $\begin{array}{l}\text { Fuel consumption (grams } \\
\text { c/KWh) }\end{array}$ & $340-420$ & $280-320$ \\
\hline Thermal efficiency (\%) & 32 & $40-45$ \\
\hline $\begin{array}{l}\text { Carbon emission rates per } \\
\text { unit generated (kg } \\
\mathrm{CO}_{2} \mathrm{e} / \mathrm{KWh} \text { ) }\end{array}$ & $0.94-1.16$ & $0.78-0.89$ \\
\hline Capital costs (USD/KW) & & $1,200-1,400$ \\
\hline O\&M Costs (USD/KWh) & 0.25 & 0.29 \\
\hline
\end{tabular}

Substantial reductions in both emissions and coal consumption could be achieved by moving towards CCT in China. For example, if $25 \mid \%$ of the 1,500 TWh of electricity generated from coal in 2003 was from super-critical pulverized coal generation units (SCPV), this would achieve a $4 \%$ (or 20 million tonne) reduction in the approximate 530 million tonnes of coal consumed for electricity generation.

The various types of CCT are at different stages of advancement in China (Zhufeng et al., 2001): coal washing, blending, and circulating fluidized bed combustion have been put into commercial operation; supercritical units and large coal gasification

\footnotetext{
${ }^{9}$ Sub critical unit fuel consumption rates are based upon the range for the different provinces as given in China's Electricity Power Yearbook. Therefore, this assumes that the fuel consumption rates in the Yearbook are mainly reflective of sub critical units.
} 
technology are under development; coal liquefaction and related generating technologies (including integrated gasification combined cycle) are mainly imported from abroad but have entered into commercial demonstration; while the retrofit of small-medium industrial boilers and other retrofit programs have been more broadly implemented.

The promotion of CCT is China is driven largely by policies focused on technology, although these policies are complemented by environmental regulations that help encourage the implementation of more efficient electricity generation technologies. Nonetheless, there remains a general lack of coordination on increasing CCT on a broad scale in China (Zhufeng et al., 2001). There are few financial incentives for the commercial development of new and key technologies. This is linked directly to the lack of effective monitoring and low participation rates of small to medium scale generators in terms of both emission reductions and CCT implementation, despite the fact that these generators are the most pollution intensive in China. The future rate of uptake of CCT therefore will be determined by proper incentives for their development (including preferential lending rates and a cohesive plan to develop domestic production capacity) and programs to encourage CCT implementation by small scale electricity generators.

\section{Combined cycle natural gas}

The primary technology available to generate electricity from natural gas is the combined cycle natural gas turbine (CCGT). This technology uses thermal energy from natural gas combustion to first drive a turbine generator and then uses waste heat from this initial process to generate steam to drive a steam turbine. Since this technology can be rapidly brought on and off-line, it is commonly used for peak-loads in the daily electricity load curve in economies with well-developed gas infrastructure. CCGT generators operate at 
higher efficiencies than typical coal-fired boiler units, with the average net energy conversion rates of 40 percent or higher. Since natural gas produces half the amount of $\mathrm{CO}_{2}$ than coal and negligible amounts of $\mathrm{SO}_{2}$, far less pollution occurs per unit of electricity generated.

In the past, this technology was not widely employed in China since natural gas was not accessible and because natural gas was priced far higher than coal. However, this is expected to change with the development of natural gas pipelines, LNG infrastructure, and any concurrent escalation in peak-load electricity demand.

\subsubsection{Emission limits}

In order to control emissions of $\mathrm{SO}_{2}$, the central government has implemented a series of measures to reduce the sulphur content of coal, improve generator efficiency, and establish emission targets and tariffs (Hao et al., 2001). These central environmental targets are important in a deregulating market such as China's as operators will chose the least-cost generation configuration after considering the added costs of pollution control or of any fines paid.

In China's 9th Five-Year Plan (1995 to 2000), national $\mathrm{SO}_{2}$ emissions where to be reduced to 1995 levels by 2000 (SEPA, 1995). By the end of the 10th Five-Year Plan (2005), $\mathrm{SO}_{2}$ emissions were to be $10 \%$ below 2000 for the nation as whole, and $20 \%$ below 2000 levels for regions falling within 'two control zones' (TCZ) (SEPA, 2002). China established the TCZ in its $9^{\text {th }}$ five-year plan in order to curb emissions in areas plagued by atmospheric sulphates and acid rain. Emission reductions will most certainty become even more stringent into the future as the size of the generation sector increases and the government likely facing even greater pressure to reduce air pollution. 


\subsubsection{Decision nodes}

The variety of policies that will affect the drivers of the future development of the electricity supply sector are complex and often overlap. Similar to electricity demand, it is useful to organize these into a set of decision nodes, whereby each is assessed from the perspective of its impact on a specific driver (see table 16):

Table 16. Decision nodes in the electricity supply sector

\begin{tabular}{|c|c|c|}
\hline Driver & Relevance to electricity supply & Associated policy areas \\
\hline $\begin{array}{l}\text { - Building of the } \\
\text { natural gas } \\
\text { infrastructure }\end{array}$ & $\begin{array}{l}\text { Increase the accessibility to } \\
\text { natural gas }\end{array}$ & $\begin{array}{l}\text { Securing adequate } \\
\text { financial resources }\end{array}$ \\
\hline $\begin{array}{l}\text { - Building a nuclear } \\
\text { power base }\end{array}$ & $\begin{array}{l}\text { Change proportion of nuclear } \\
\text { power in the electricity mix. } \\
\circ \text { Decrease emissions and } \\
\text { fossil-fuel requirements }\end{array}$ & $\begin{array}{l}\text { - Central governments } \\
\text { nuclear aspirations and } \\
\text { ability to raise } \\
\text { necessary capital } \\
\text { internally } \\
\end{array}$ \\
\hline $\begin{array}{l}\text { - Implementation of } \\
\text { the REL }\end{array}$ & $\begin{array}{l}\text { - Increases the proportion of } \\
\text { renewables in the electricity mix }\end{array}$ & $\begin{array}{l}\text { Decisions on regional } \\
\text { circumstances and } \\
\text { targets }\end{array}$ \\
\hline $\begin{array}{l}\text { - Fuel availability } \\
\text { and price }\end{array}$ & $\begin{array}{l}\text { - Fuel competitiveness } \\
\text { - Supply structure }\end{array}$ & $\begin{array}{l}\text { - Attracting sufficient } \\
\text { capital resources and } \\
\text { investment } \\
\text { - Liberalizing primary } \\
\text { fuel markets }\end{array}$ \\
\hline $\begin{array}{l}- \text { Changes in } \\
\text { technology }\end{array}$ & $\begin{array}{l}\text { - Increase efficiency } \\
\text { - Reduce pollutant emission rates } \\
\text { - Reduce capital costs }\end{array}$ & $\begin{array}{l}- \text { R\&D policies } \\
\text { - Environmental } \\
\text { policies }\end{array}$ \\
\hline $\begin{array}{l}\text { - Environmental } \\
\text { regulations }\end{array}$ & - Impacts on the cost of generation & $\begin{array}{l}\text { - Emission limits and } \\
\text { tariffs }\end{array}$ \\
\hline
\end{tabular}

\subsection{Development of a conceptual framework}

A review has been provided of factors that have influenced and will play a future role in the GHG emissions resulting from the generation of electricity in China. This review has 
provided the basis for a conceptual framework (presented in figure 12) for modelling the sectors future development:

Figure 12. A conceptual framework for modelling the development of China's electrical sector

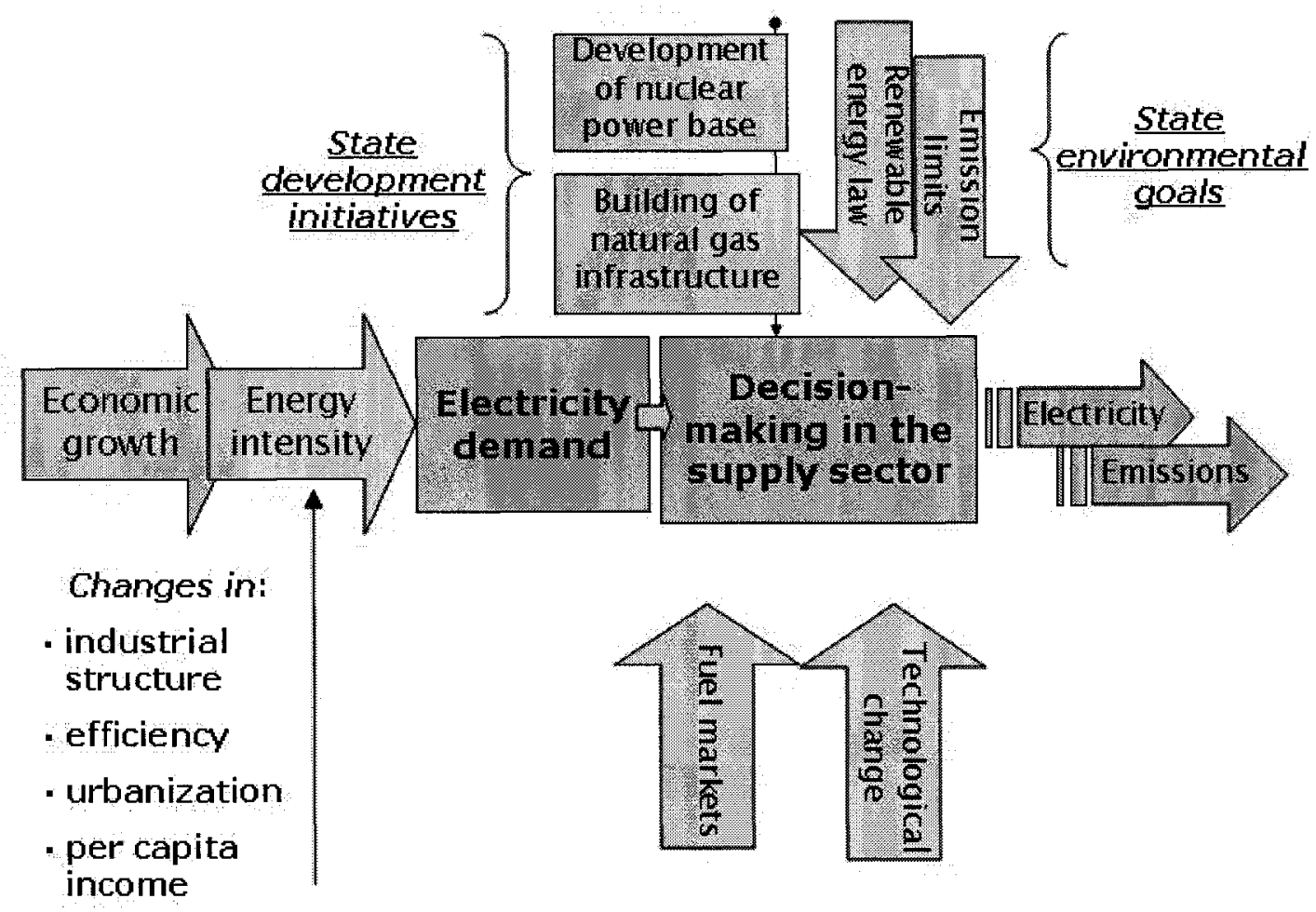

Fundamental to demand are changes within the economy. This influences and is influenced by processes of industrialization, restructuring, urbanization and changes in per capita income. Electricity supply, meanwhile, must be considered in the context of parallel state development initiatives and environmental goals, and processes underlying fuel price and technological change. 
A number of key drivers are highlighted. On the demand side, economic growth is widely expected to remain strong and per capita incomes to rise commensurately. Coupled with continuing urbanization, the growing wealth will drive electricity demand higher. In the supply sector, investment decisions on the type of technology and fuel source mix will be strongly influenced by expectations regarding the prices of underlying fossil fuels and any emission restrictions implemented by the central government. Development initiatives being undertaken by the central government will also continue to play a critical role. China is trying to develop a natural gas infrastructure and is continuing along an ambitious nuclear energy program. At the regulatory level, the central government will be requiring final electricity suppliers to purchase certain percentages of electricity from renewables.

There are a number of uncertainties that will influence the development path of China's electrical sector in the context of the above drivers. The first lies in the stability of economic growth and possibilities for both reduced economic output and higher interest rates as this would affect both the demand for electricity and the financing of new electricity capacity. On the supply side, the largest uncertainty likely is the relative price of coal to natural gas. With the supply and demand issues highlighted, China is becoming more integrated with international energy markets, and more susceptible to price fluctuations as a result. A rapid rise in natural gas price would mean coal would dominate the electricity generated in China. Technological change will also be crucial for reducing the environmental impacts of electricity generated. 


\subsection{Future scenarios}

How might the drivers and processes included in the conceptual framework shown in figure 12 develop into the future? Three scenario storylines are envisioned, each containing different trajectories in the drivers included in the conceptual framework to reflect different possible routes of development. These storylines are developed by following a two-dimensional framework that involves envisioning how the global economic system may look if processes of globalization are emphasized versus one where regulation increases, and differing worlds when considering if economic growth is promoted at the expense of environmental protection, and vice versa (see figure 13).

\section{Figure 13. Two-dimensional framework for scenario setting (derived from the IPCC (2000a))}

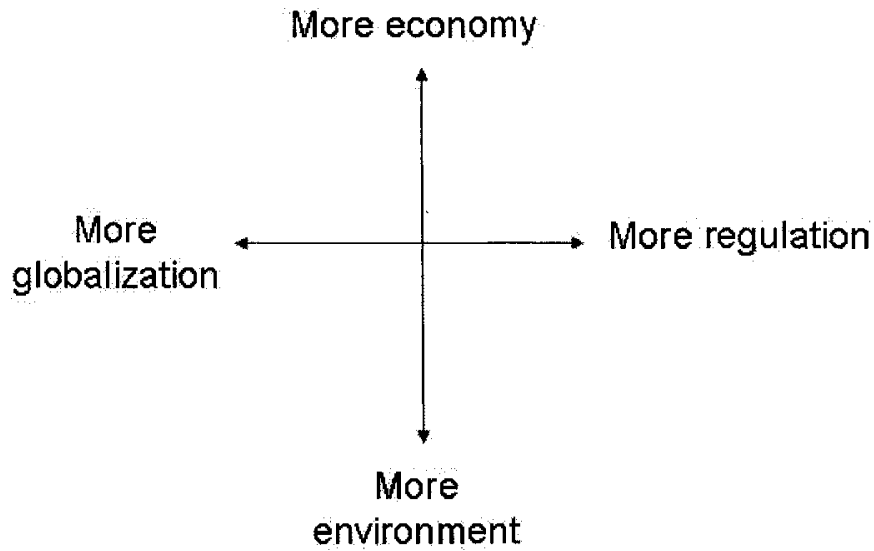

This general approach follows the scenario generation exercise conducted by the IPCC (2000a) where four families of scenarios were created (i.e. the A1, A2, B1, and B2 scenario families). The A1 family existed within a world with high rates of globalization and economic growth; the A2 family existed within a highly heterogeneous world based 
on self reliance and not favoring globalizing; the B1 family follows the population and economic growth characteristics of the A1 family, but with a world economy that restructures to a more environmentally benign service and knowledge based one; and the B2 family of scenarios which follows the basic tenets of the B1 scenario family but where there is greater emphasis is on local solutions to environmental problems. In this research only three scenarios were created as this was the limit put in place by ICF in terms of the number of scenarios they were willing to generate.

The three scenarios included relate to figures 13 and 14 as follows. The first scenario can be considered the BAU scenario (similar to the A1 family of scenarios by the IPCC). It is characterized by a Chinese government that places more emphasis on globalization versus regulation, and a China that encourages economic growth over environmental protection. Even so, this BAU scenario embraces the environmental goals that the PRC has established. The second scenario is characterized by greater regulation in China's economy, but an emphasis still on economic growth (with features similar to the A2 scenario family established by the IPCC) and is subsequently referred to as the 'conservative scenario'. This emphasis on regulation is largely a reaction to global economic and political events. The third scenario, referred to as the 'optimistic' scenario, is characterized by a China that is able to greatly benefit from a world that becomes even more globalized than that seen under the BAU, a China that emphasizes economic growth but is able to benefit from factors that contribute to lessening the environmental intensity of economic activity through some of the benefits received from increased global trade and a more service based global economy (with features similar to the B1 scenario family established by the IPCC). 
The range in values that originate from these storylines are presented and compared in tabular format and then placed relative to the two-dimensional framework for scenario setting presented in figure 13 (see table 17 and figure 14), with the global and domestic contexts of these scenarios then summarized in the sub-sections that follow.

Table 17. General socio-economic and technological attributes of scenarios

\begin{tabular}{|c|c|c|c|}
\hline & Business as usual & Conservative & Optimistic \\
\hline \multicolumn{4}{|c|}{ Rate and direction of change in driver and components } \\
\hline Economic growth & Moderate increase & Slow increase & $\begin{array}{l}\text { Moderately-fast } \\
\text { increase }\end{array}$ \\
\hline Energy intensity & Steady throughout & Steady throughout & $\begin{array}{l}\text { Steady, then slow } \\
\text { decrease }\end{array}$ \\
\hline $\begin{array}{r}\text { Industrial } \\
\text { structure }\end{array}$ & $\begin{array}{l}\text { Heavier weighting in } \\
\text { heavy industries }\end{array}$ & $\begin{array}{l}\text { Slowdown in } \\
\text { manufacturing }\end{array}$ & $\begin{array}{l}\text { Broader based } \\
\text { economic growth }\end{array}$ \\
\hline Per capita income & Moderate increase & Slow increase & Fast increase \\
\hline Urbanization & Moderate increase & Slow increase & $\begin{array}{l}\text { Moderate } \\
\text { increase }\end{array}$ \\
\hline $\begin{array}{c}\text { Main driver: } \\
\text { Electricity demand }\end{array}$ & Moderate & Moderately slow & Moderately fast \\
\hline $\begin{array}{r}\text { Natural gas } \\
\text { infrastructure }\end{array}$ & $\begin{array}{l}\text { Success (steady } \\
\text { construction) }\end{array}$ & $\begin{array}{l}\text { Insufficient funds } \\
\text { (construction limited) }\end{array}$ & $\begin{array}{l}\text { Success (rapid } \\
\text { construction) }\end{array}$ \\
\hline $\begin{array}{r}\text { Renewable energy } \\
\text { law }\end{array}$ & $5 \%$ by $2010,10 \%$ by 2020 & $\begin{array}{l}2 \% \text { by } 2010,5 \% \text { by } \\
2020\end{array}$ & $\begin{array}{l}5 \% \text { by } 2010,12 \% \\
\text { by } 2020\end{array}$ \\
\hline Emission limits & $\begin{array}{l}\text { Successful } \\
\text { implementation of current } \\
\text { policy }\end{array}$ & $\begin{array}{l}\text { Emission limits are } \\
\text { met for } \mathrm{SO}_{2} \text {, but } \mathrm{CO}_{2} \\
\text { emission intensities } \\
\text { rise }\end{array}$ & $\begin{array}{l}\text { Exceed current } \\
\text { policy goals }\end{array}$ \\
\hline Nuclear power & $\begin{array}{l}\text { Successful meeting of } \\
\text { development goals }\end{array}$ & $\begin{array}{l}\text { Insufficient funds } \\
\text { (construction limited) }\end{array}$ & $\begin{array}{l}\text { Successful } \\
\text { meeting of } \\
\text { development } \\
\text { goals }\end{array}$ \\
\hline $\begin{array}{r}\text { Price of natural } \\
\text { gas }\end{array}$ & $\begin{array}{l}\text { Low-moderate rise to } \\
2015 \text {, moderate rise } \\
\text { thereafter }\end{array}$ & $\begin{array}{l}\text { Prices remain strong } \\
\text { throughout, } \\
\text { availability limited }\end{array}$ & $\begin{array}{l}\text { Low rise to } 2015 \text {, } \\
\text { moderate rise } \\
\text { thereafter }\end{array}$ \\
\hline Price of coal & Low rise throughout & Low rise throughout & $\begin{array}{l}\text { Low rise } \\
\text { throughout }\end{array}$ \\
\hline $\begin{array}{r}\text { Technological } \\
\text { change }\end{array}$ & Moderate & Slow & Moderately-fast \\
\hline
\end{tabular}


Figure 14. Placing the scenarios in a two-dimensional framework for scenario setting

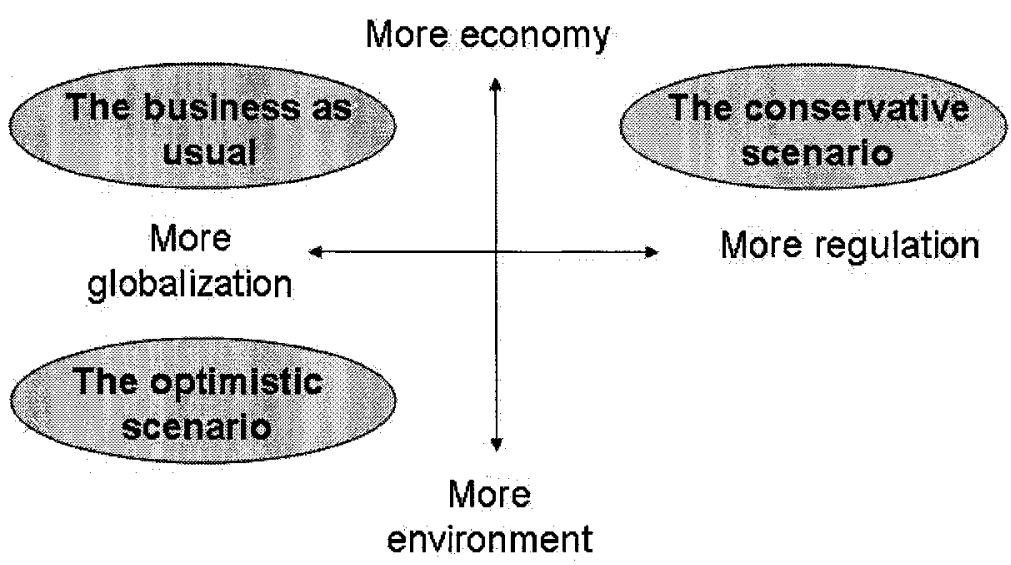

\subsubsection{The 'business as usual' scenario}

The 'business as usual' scenario is characterized by economic growth as set in official targets given in China's national economic plans, as well as success in the implementation of State development and environmental goals.

This scenario takes place in a world that continues to become globalized and a global economy that experiences moderate growth throughout the period considered. There is relative political stability throughout the world as nations strive to maintain open economic relations. With continued economic growth, however, the demand for 
commodities and energy resources accelerates and this increases the competition for and prices of these. Global economic efficiencies are gained from the ability to use regional comparative advantages, including the continued use of China and Asia's low cost labour force. These processes also result in continued inflows of foreign direct investment (FDI) and technology from the developed world to less developed nations. Global trade is high, especially for manufactured goods, and with advances in global trade, technological innovation is robust, greater efficiencies are made and energy intensities fall throughout both industrialized and industrializing nations. Within the general rise in global economic output, the economies of developing nations grow faster relative to more mature economies as these catch up in terms of technology and because of their higher rates of population growth.

China in particular is able to benefit from the above world. Its economy continues to open to foreign investment and the manufacturing sector sees strong inflows of FDI in initial years. As a result, its economy is able to maintain growth rates of 7 to 7.5\% per annum until 2020. Because of rapidly increasing economic activity, per capita income rises to be near 10,000 USD per annum by 2010 , and 15,000 USD per annum by 2020. Although much of this is tied to continued growth in manufacturing industries initially, the central government is able to steer the economy to a more broadly based one over time, particularly where personal consumption plays a much more important role. Urbanization also continues in China, and by 2020 , nearly $65 \%$ of the population lives in urban areas. The above processes combined result in economic restructuring as seen in recent years. Heavy industrial sectors continue to ramp up production to meet requirements for infrastructure construction and to meet the increasing domestic demand 
of consumer good items which require inputs derived from these industries. These events result in a consistent rise in electricity demand, particularly at peak hours in the daily load curve.

There are a number of structural issues that have implications for the medium to long term economic growth and stability in China that are successfully dealt with. China is able to continue to reform its many overstaffed and inefficient State-owned enterprises. In addition, it is able to successfully overhaul its banking system that is current carrying a sizeable proportion of non-performing loans. Many of these reforms are enabled by China's membership in the World Trade Organization as this forces the government to pursue these reforms, as well as a continued process to open its banking sector to foreign involvement.

In the electricity supply sector, the government is able to successfully meet many of the relevant policy objectives it has outlined in recent years. In particular, it is successfully able to increase the proportion of renewables in its electricity sector to $5 \%$ of total national capacity by 2010 , and $10 \%$ by 2020 . Due to the consistency and strength of its economic growth, it is able to finance and develop the nuclear power base it has set out to build. Similarly, but with broader implications on the electricity supply sector, a stable banking sector and increased contributions of investment by major multi-national energy corporations allow the natural gas infrastructure to be quickly constructed. In eastern regions a number of LNG terminals are built by 2010, and long- term supply contracts are signed at reasonable prices. The WEP is also able to tap into natural gas sources in Kazakhstan and other former Soviet states, while a major pipeline is constructed running from east Siberia, through northern China and ending in a liberalized 
and more politically stable North and South Korea. The consequences of the above for the electricity sector is increased accessibility to and increasing cost competitiveness of natural gas for electricity generation, particularly in years before 2015. However, after this point, increasing global demand for natural gas results in increased upward price pressures. Emission limits are also met for $\mathrm{SO}_{2}$ under this scenario.

There is also significant technological change that occurs within the supply sector due to the adoption of policies established specifically to stimulate cleaner generation technologies, domestic efforts to develop production capabilities, and increasing competitiveness and direct foreign investment into the sector. In particular, there is greater adoption of CCT and natural gas combined cycle generation plants. The successful adoption of these is encouraged by decreasing capital costs as domestic production capacity increases.

\subsubsection{The 'conservative' scenario}

In the 'conservative' scenario economic growth occurs at a tempered pace with the emission intensity of electricity generation higher per unit of economic output due to a higher contribution of coal and lower technological development. The scenario is conservative with respect to its assumptions concerning economic growth, the ability of the Chinese to increase technological development, and the ability to move away from coal as a primary fuel source.

This scenario takes place in a world very different than the one painted for the BAU. A major contributor to this world are fractures that emerge within the American economy due to the rapidly rising trade and budget deficits experienced since 2000 , and increasing personal debt levels. Particularly, the U.S. currency experiences a steep fall in 
response to trade imbalances and decreased international investor interest. Interest rates rise, and problems emerge within the housing market. Consumption falls, and the U.S. economy enters into a prolonged recessionary period. In response, the U.S. government becomes more protectionist, seeking to rebuild sectors drained by previous processes of globalization, with trade barriers enacted in sub-sectors that include manufacturing, communications, and 'special advanced technology products' (ATP) ${ }^{10}$. There is decreased trade and information flow under this scenario, and reduced flows of FDI into China. One repercussion of this is that the technological characteristics of the electricity sector do not advance as fast as under the BAU. Because the world is not characterized by a strong desire to maintain close economic relations, this affects political stability throughout the world, giving rise to numerous conflicts. This political instability in turn reduces global economic growth and leads to price premiums on energy that must be supplied from politically unstable regions. In addition, there are escalating tensions over China's requirements for oil, gas and coal on world energy markets, and of China's ambitions to secure supply sources for them. A number of countries compete to secure supply of energy sources, resulting in a further price premium being paid for these fuels on international markets.

Domestically, China is unable to maintain the rapid economic growth experienced throughout the late 20 th and initial parts of the 21 st centuries. Since $20 \%$ of China's exports are to the U.S., and with the American economy fundamental to the strength of

\footnotetext{
${ }^{10}$ In the late 1980 's, a special category was created in U.S. trade statistics to show the contribution of high technology products to total trade. In 2001, this index went negative. An important contributor to the U.S. trade deficit of 30 billion USD in this trade category that has been experienced in most recent years has been China: in 2003, the U.S. - China trade deficit in this category equaled 21 billion USD, a dramatic change from the trade balance experienced in 1998 (Prestowitz, C. (2005). Three Billion New Capitalists. New York, Perseus Books Group.
} 
exports to other countries in the global supply and production chain as well, America's economic problems reverberate around the world and in China. This not only reduces China's ability to achieve the high rates of economic growth established under its five year plans, but also negatively impacts the country's ability to finance its ambitious nuclear and natural gas infrastructure programs. Consequent to the decrease in domestic economic growth, income growth is also tempered, resulting in less purchasing power than in the BAU, and thereby decreasing consumption of electrical appliances and lowering residential sector electricity demand than otherwise would have occurred.

On the supply side of the electricity sector, there are a number of important differences from the BAU. First, due to decreased financing ability, domestic targets are not met for either building of the nuclear power base or construction of the natural gas infrastructure. This, in conjunction with international high prices for energy (particularly oil and natural gas), means that the proportion of coal used for electricity generation remains high. In a similar vein, there is a decrease in technological innovation in China's electricity supply sector, and efficiencies are not gained as under the BAU.

\subsubsection{The 'optimistic' scenario}

This scenario takes place in a world where globalization processes accelerate. Similar to the BAU, efficiencies are gained from the successful allocation of each aspect of the production process across the global sphere and, as a result, with significant global benefits received from regional competitive advantages. The flow of FDI and technology from the developed world to less developed nations increases alongside heightened rates of global trade. Global economic output accelerates in developing nations as they catch up to the more developed nations, while concurrently advances in information and energy 
technologies quicken resulting in a heightened reduction in energy intensities throughout the world. Global energy supply and international energy markets remain secure partially due to the co-operation of major importers, including the U.S, China and Europe, in maintaining political stability in source regions. Politically, global peace and stability means that domestic economies can specialize in areas of competitive advantage while also ensuring that each component of the value chain is both accessible and low cost for domestic production and consumption requirements. The American economy also achieves stable economic growth, with budget deficits shrinking and trade deficits declining with moderating consumption and a re-establishment of American dominance in areas of high technology and finance.

China is able to benefit immensely from the above world. Its economy continues to open to foreign investment and its manufacturing sector sees strong inflows of FDI in initial years. Its economy diversifies successfully, and strong industries are created in higher value added sub-sectors, including automotive, telecommunications, and computer manufacturing. As a result, its economy is able to maintain growth rates of 8 to $9 \%$ until 2010, lowering slightly thereafter. Because of rapidly increasing economic activity, per capita income rises. However, because much of this is tied to continued growth in manufacturing industries located in urban areas, income disparities in China remain. Nonetheless, incomes rise in rural areas as labour productivity increases, partially in response to the lower rates of population growth in these regions.

In the supply sector, there is a faster uptake of CCT and other advanced generation technologies than under the BAU. The central government is able to meet internal development targets for the construction of both natural gas infrastructure and the 
nuclear power base. Furthermore, strong economic growth globally accelerates the demand and price for energy commodities. This helps trigger further technological advances and efficiency gains, including an increased role of fuel cell technology in the generation sector. 


\section{Scenario generation}

Synopsis: In chapter 6, a conceptual framework was developed that can be used to help understand the future development of China's electrical sector. This framework embraces key economic, regulatory, social, and policy regimes which will help determine the sector's future development, most operating in some sort of interactive or complimentary way. Assurance that this framework embraces the most important technoeconomic and socio-economic factors has been gained from the research and analysis that preceded its construction, including the information that was provided by the two decomposition analyses completed. Following, three storylines were envisioned of future possible development paths for China's electrical sector. In this chapter, these storylines are now taken and actual scenario analysis is completed. There are two baseline methods where such scenario analysis can take place: decomposition and dispatch analysis. However, because of their technical and methodological underpinnings, each baseline method must use the storylines painted in different ways. Decomposition is constrained in that it only is able to consider the factors analyzed historically. Dispatch modelling, alternatively, must focus upon the interacting factors and constraints contained in the model.

\subsection{Chapter introduction}

In this chapter, the scenario storylines envisioned in chapter 6 are used to generate scenarios of electricity demand, supply, and the associated emissions of GHGs to the year 2020. This analysis involves two components. The first part of the chapter presents the scenario analysis using the decomposition baseline method. The scenarios are generated 
following the general storylines painted in chapter 6 , but where only the factors considered in the historical decomposition analysis are manipulated. The second part of the chapter contains scenario analysis using dispatch modelling. Again, the general storylines painted in chapter 6 are followed. However, rather than the analysis being restricted to what was considered in a historical context, the analysis embraces the range of drivers interacting within the dispatch model under the premise of mathematical optimization when there is an objective to reduce costs. Therefore, this chapter contains two fundamentally different ways of generating scenarios, even if these utilize the same general storylines.

\subsection{Generating scenarios using decomposition analysis}

\subsubsection{Using decomposition analysis in conjunction with scenario storylines}

How can the results from the decomposition analysis be used in conjunction with the storylines envisioned for the possible future development of China's electrical sector? The results of the decomposition analysis helped to quantify the importance of several key factors in determining changes in the emission intensity of electricity generated in China. It is these factors that are considered in terms of the various scenario storylines, particularly in terms of how they might change under each storyline.

It was identified that increases in efficiency was the most important factor affecting changes in both electricity demand and the emission intensity of electricity generated in China. Under the business as usual, efficiency in the demand and supply sectors improves into the future. Improvements are also made in reducing line loss and 
the auxiliary use of power, inline with the storyline underlying the business as usual scenario. There are also increases in nuclear and natural gas generation in this scenario, although coal generated electricity continues to dominate. Alternatively, these factors vary in both the conservative and optimistic cases according to the underlying trajectories set out in the storylines. In the conservative case, efficiency does not improve as quickly, but neither does economic growth. Conversely, in the optimistic case there is higher economic growth, but also higher increases in efficiency, development of nuclear power, and improvements in transmission technology.

\subsubsection{Scenario generation}

The application of the storylines in establishing scenarios for China's electrical sector is presented in terms of both the emission intensity and the emissions associated with the electricity generated in each scenario (see table 18 and figure 14).

Table 18. $\mathrm{CO}_{2}$ emissions from electricity generation under different scenarios

\begin{tabular}{|c|c|c|c|}
\hline \multicolumn{4}{|c|}{ Emission intensity $\left(\mathrm{kg} \mathrm{CO}_{2} / \mathbf{k W h}\right)$} \\
\hline 1980 & 1.25 & $N / A$ & $N / A$ \\
\hline 2002 & 0.95 & $N / A$ & N/A \\
\hline 2010 & 0.86 & 0.93 & 0.84 \\
\hline 2020 & 0.76 & 0.91 & 0.72 \\
\hline
\end{tabular}

\begin{tabular}{|c|c|c|c|}
\hline \multicolumn{4}{|c|}{ Emissions $\left(\mathrm{MTCO}_{2}\right)$} \\
\hline & & "Conservative" & "Optimistic" \\
\hline 1980 & 319 & $N / A$ & N/A \\
\hline 2002 & 1,345 & $\mathrm{~N} / \mathrm{A}$ & N/A \\
\hline 2010 & 2,120 & 2,247 & 2,117 \\
\hline 2020 & 3,910 & 4,482 & 3,807 \\
\hline
\end{tabular}


Figure 15. Decomposition of carbon emission intensity - BAU scenario

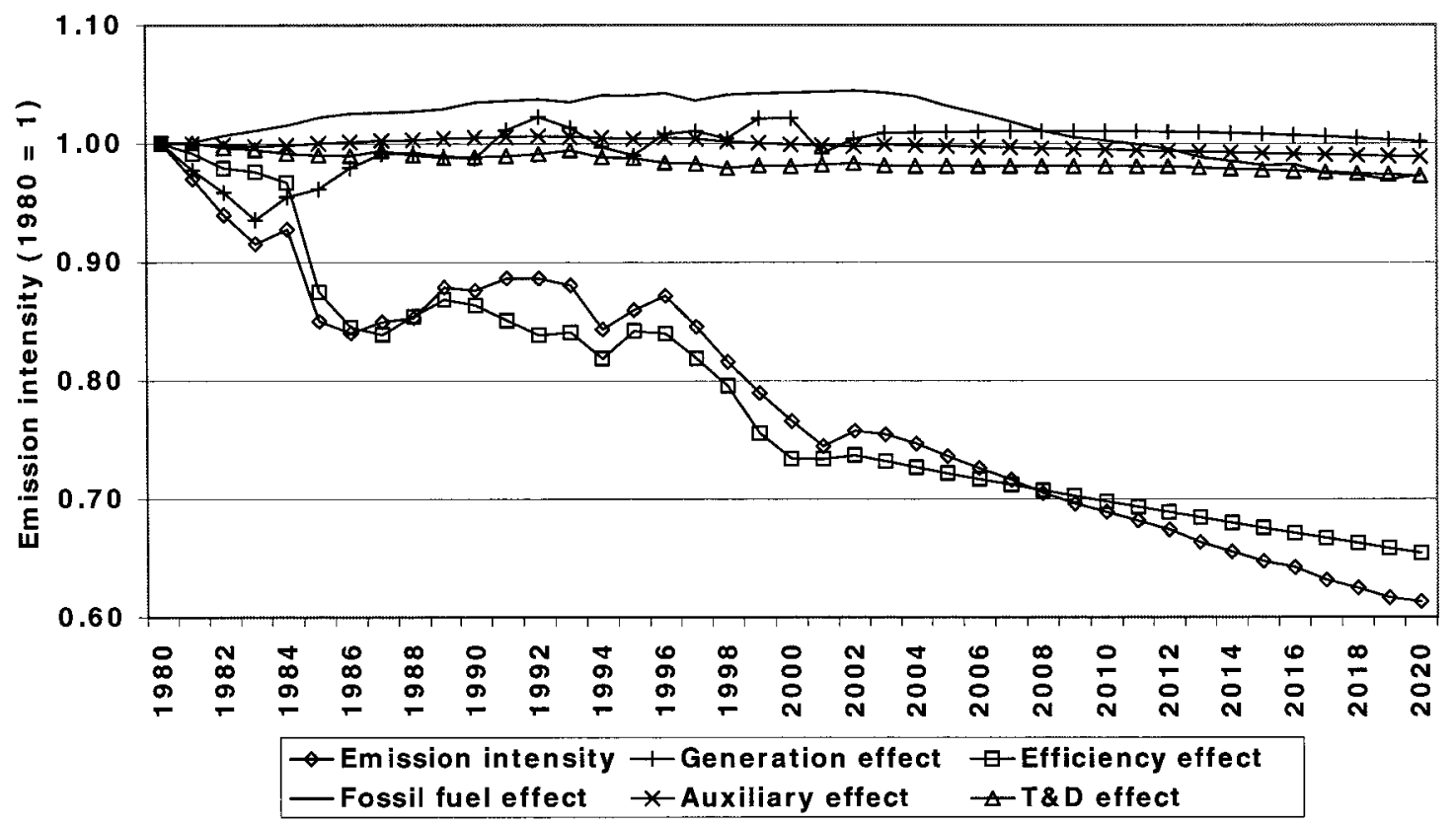

Under the BAU scenario, the carbon emission intensity decreases to approximately $62 \%$ of 1980 levels by 2020 . There are three primary factors contributing to this: efficiency increases in thermal generation, increases in nuclear generation and increases in the share of natural gas as a primary fuel in thermal electricity generation. Comparing the BAU against the conservative and optimistic cases (see figures 15 and 16), the assumptions followed in the former of these would result in a greater emission increase than the emissions reduction achieved in the latter. The reason for this is the lack of uptake of nuclear or natural gas in the conservative scenario. 


\section{Figure 16. Decomposition of carbon emission intensity - Conservative scenario}

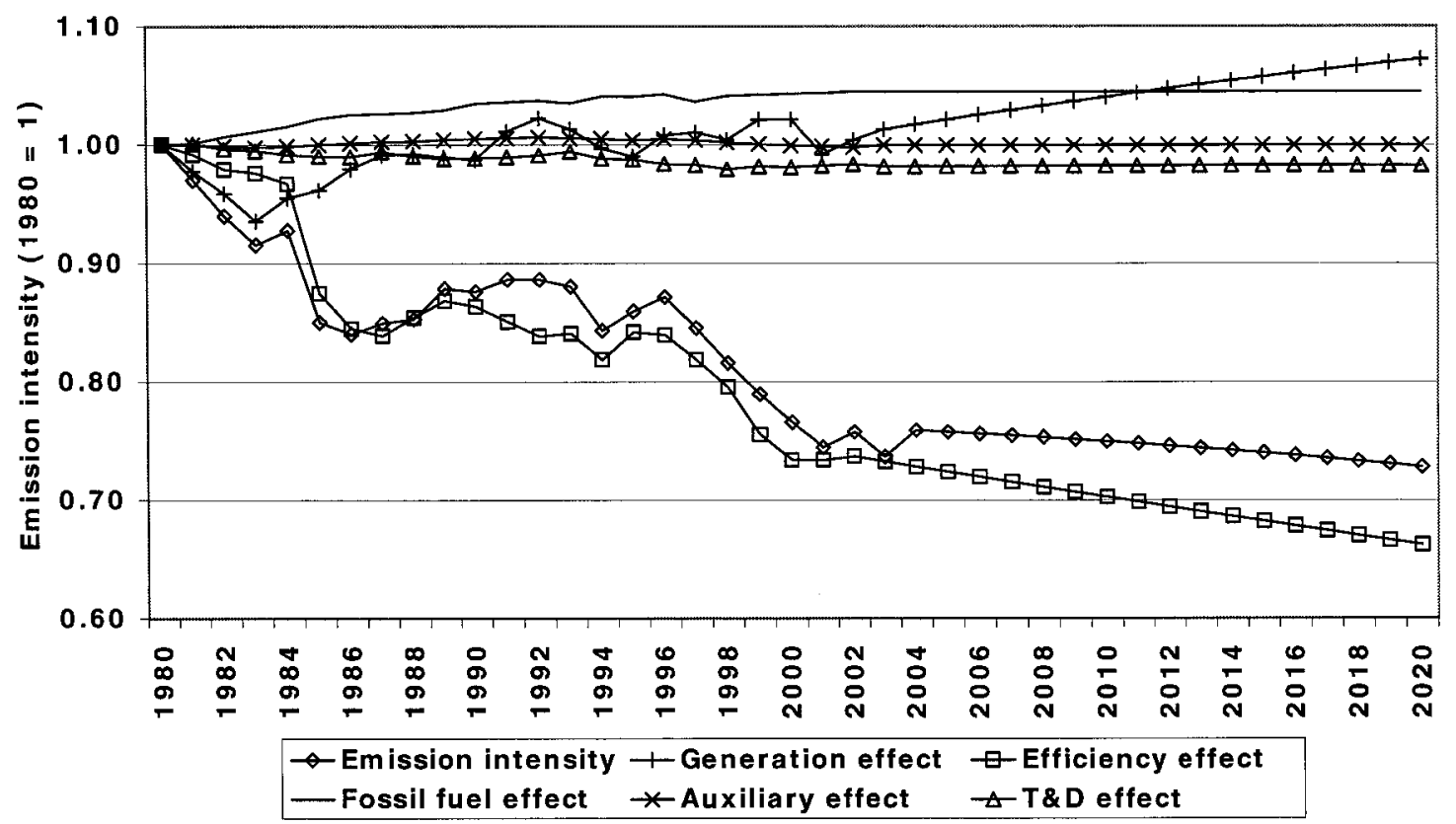

The conservative scenario demonstrates the effects of not developing either natural gas or nuclear and the effects of a decrease in efficiency improvements of thermal generators. The overall impact of these events would mean an emissions intensity of $66 \%$ of 1980 levels by 2020 . Here, the most significant factor leading to an increase in the emission intensity relative to the $\mathrm{BAU}$ is the decrease in nuclear power generation. 
Figure 17. Decomposition of carbon emission intensity - Optimistic scenario

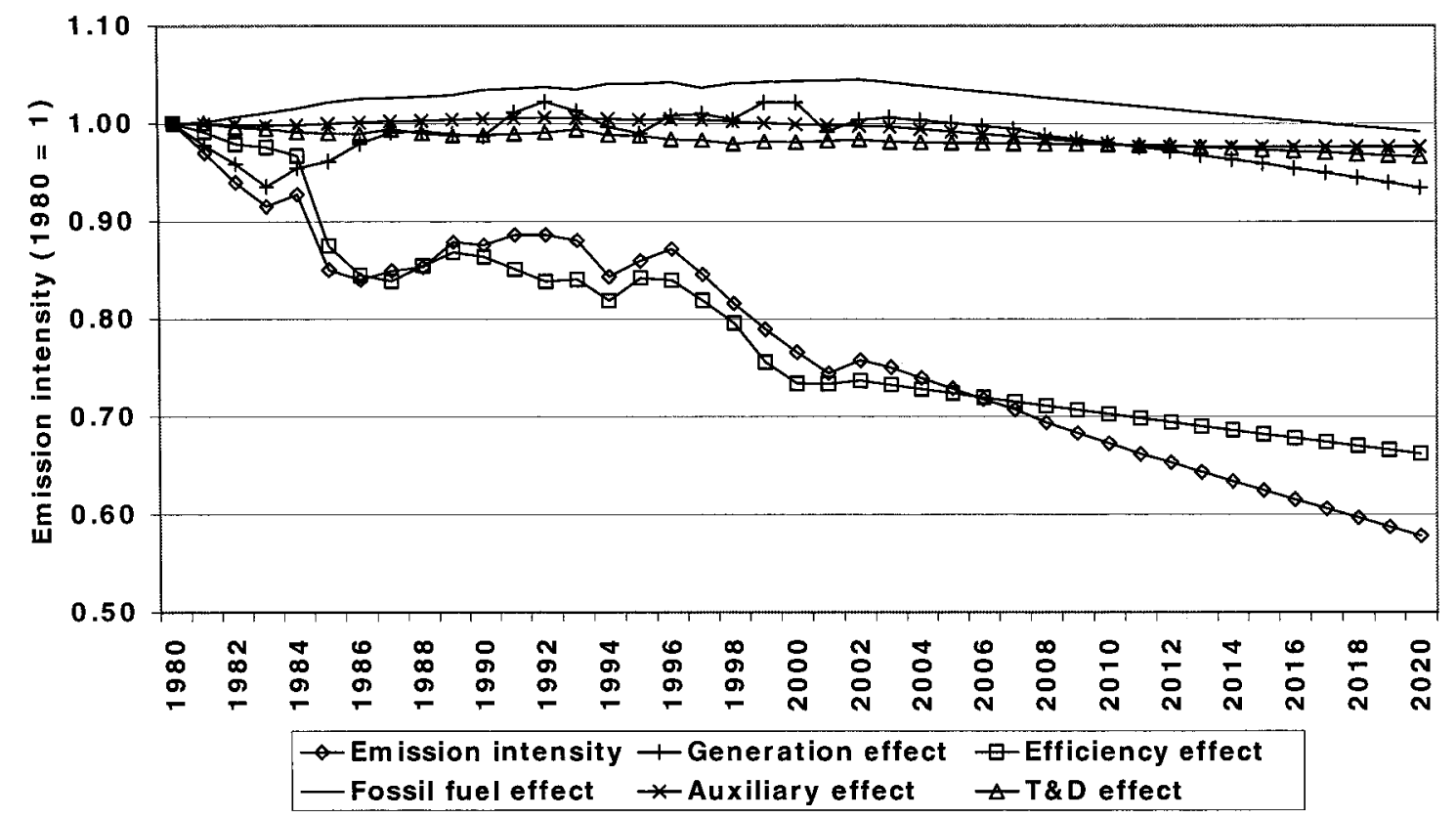

The optimistic scenario assumes slightly higher penetrations of coal, nuclear and efficiency gains in generation and in end use. The result of this is an emission intensity in $202058 \%$ of 1980 levels.

\subsubsection{Conclusions on using decomposition analysis and scenario generation}

Decomposition analysis has shown that between 1980 and 2002, the efficiency increases of thermal generators was the most important factor leading to decreases in the emission intensity of electricity generated in China. Under current policy initiatives and following economic processes that have occurred over the past twenty years, the emission intensity of electricity generated in China will continue to decrease into the future. Here, an important factor that emerges is the impact of increased nuclear generation and increased 
use of natural gas as a primary fuel. Both factors will lead to decreases in the emission intensity of electricity generation since they are zero or low carbon sources for electricity.

Since the analysis was focused on changes in the emission intensity of electricity generation rather than actual changes in emissions, changes in economic activity were not explicitly considered in the decomposition equations formulated. However, using decomposition analysis within a country experiencing such robust economic growth may not be able to adequately consider some economic uncertainties unless these are explicitly considered in scenarios generated. In China, these include such possibilities as resource constraints emerging in the primary fuels sector or the level of financial resources available or allocated towards capital investment and new technology.

Nonetheless, the analysis completed confirms the proposition that decomposition is an appropriate method that can be used to formulate emission baselines that in turn might be suitable for the analysis of CDM projects initiated into the electrical sector. Decomposition is also useful as it can be used to first understand historical factors that affect emission intensity, with this information useful in providing estimates of the future progression and influence of these factors on emission intensity. Finally, from this analysis it can be confirmed that decomposition is an easy to use, transparent and highly accessible approach for baseline construction, and therefore meets many of the criteria used to evaluate these.

\subsection{Generating scenarios using dispatch simulation}

Scenario analysis is now completed using the ranges in drivers associated with each scenario storyline (as was described in chapter 6) as inputs into the China Integrated Planning Model (IPM). This model was described in detail in section 3, but to reiterate 
the process of using this tool, research was conducted on a series of drivers and constraints that were used as inputs into the dispatch model. This included the following parameters:

\section{Drivers:}

- Electricity demand by province and year

- Peak-load requirements by province and year

- Fuel prices (coal, natural gas, diesel) by region and year

- Technology costs by year

- Technology efficiency by year

\section{Constraints:}

- Sulphur dioxide emission limits

- Renewable energy targets

- Constraints on the availability of fuels

- Established nuclear-powered electricity development program

These inputs were used to generate the various scenarios generated, with the values of the drivers varying according to the scenario storylines as set out in chapter 6 . The process involved sending the various inputs to the facilitators of the model at ICF Consulting, who then inputted the various drivers and constraints and ran the tool. 


\subsubsection{Scenario results using the China IPM}

The results of the scenario analysis using the China IPM is presented in terms of the electricity generated by scenario, source, and region, emissions by scenario, and the emission intensity of electricity generation by region and scenario.

Figure 18 presents the total national electricity generation by scenario as calculated by the IPM, while figure 19 presents the associated $\mathrm{CO}_{2}$ emissions by scenario. 
Figure 18. Electricity generation by scenario in the IPM

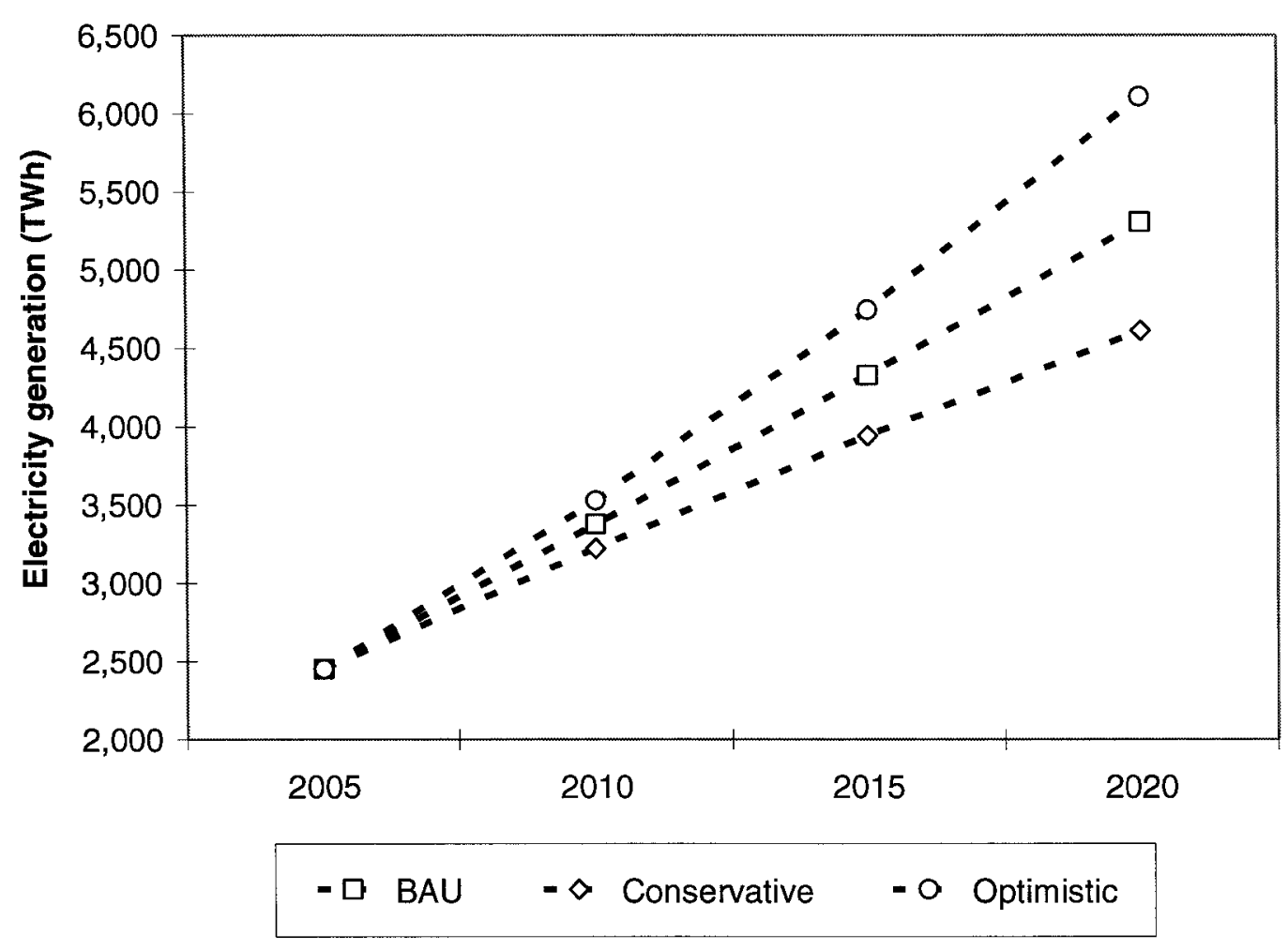

Figure 19. $\mathrm{CO}_{2}$ emissions by scenario in the IPM

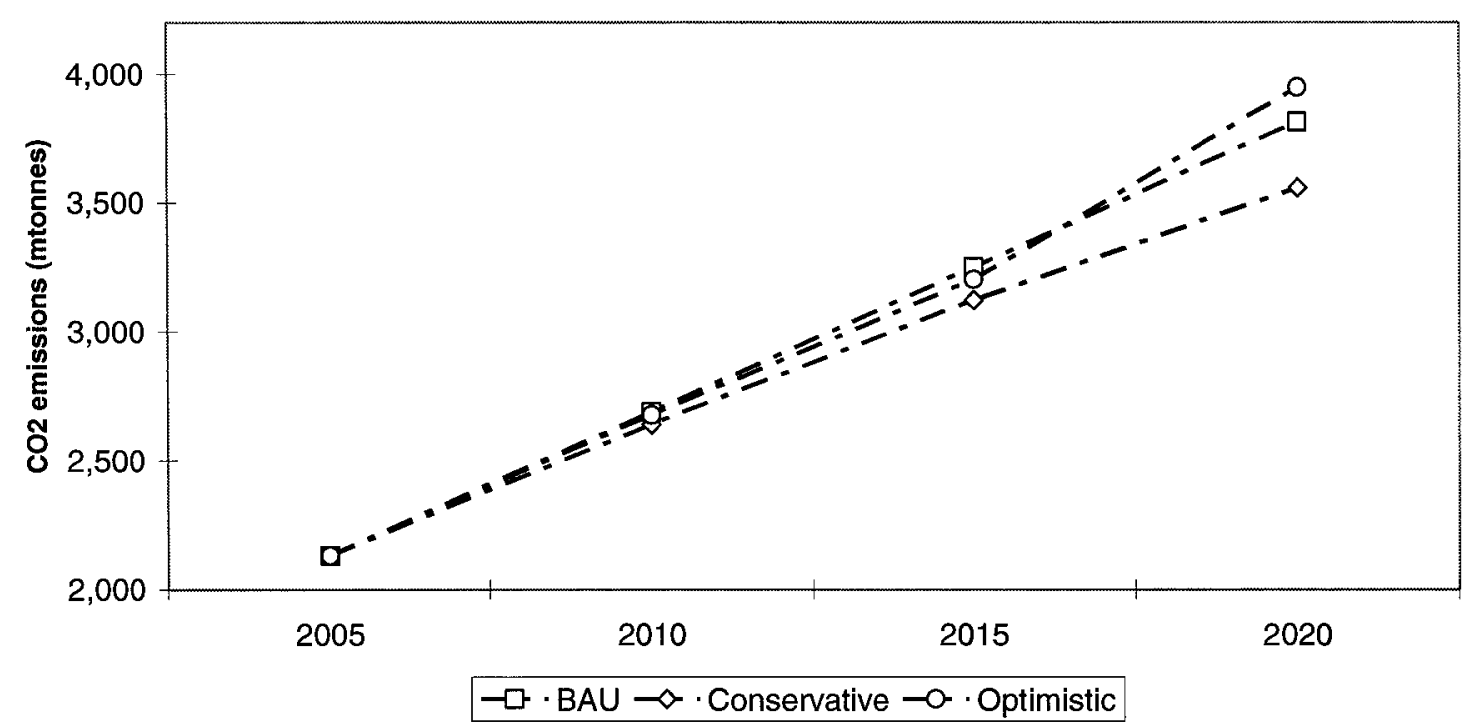


A few key points are made concerning both electricity generation and the associated $\mathrm{CO}_{2}$ emissions. Under the BAU, total electricity generation rises $37 \%$ between 2005 and 2010 to reach approximately $3,375 \mathrm{TWh}$, and by 2020 will be over twice the amount generated in 2005. Meanwhile, $\mathrm{CO}_{2}$ emissions rise at a slower pace than electricity generated, equaling approximately $25 \%$ from 2005 by 2010 , and $80 \%$ above 2005 levels by 2020 . The reasons for this divergence are continued efficiency gains in the electrical sector, and moderate fuel switches to natural gas. Alternatively, under the conservative scenario (where economic growth is lower but energy intensity higher), electricity generation is $5 \%$ below the BAU by 2010 , and nearly $15 \%$ below 2005 by 2020 . In the optimistic scenario, the opposite is the case. By 2010 , total electricity generated is approximately 4\% above the BAU and $15 \%$ above the BAU in 2020.

Comparing the conservative and optimistic scenarios with the BAU, much smaller differences are seen for $\mathrm{CO}_{2}$ emission trends than for total electricity generated. In the conservative scenario, for example, where electricity generated was $5 \%$ below the BAU in 2010 , and nearly $15 \%$ below in 2020 , for $\mathrm{CO}_{2}$ emissions these scenarios are nearly equal in 2010 , with the conservative only being $7 \%$ below the BAU by 2020 . The optimistic scenario, although having much higher growth rates for electricity generation, has $\mathrm{CO}_{2}$ emissions that remain very close to the BAU scenario until 2015 , and rising to be only $3 \%$ above BAU levels by 2020 .

Electricity generation by source by scenario

The explanation for the trends in $\mathrm{CO}_{2}$ emissions seen in figure 17(b), and particularly the differences observed when comparing growth rates for electricity generated and 
emissions, can be partially explained by the changing source mix that occurs within the IPM under these scenarios (see table 19). .

Table 19. Electricity generation by source by scenario as calculated by the IPM

\begin{tabular}{|c|c|c|c|}
\hline \multicolumn{4}{|l|}{ Business as usual } \\
\hline$\overline{\text { Coal }}$ & $80 \%$ & $80 \%$ & $80 \%$ \\
\hline Gas & $2 \%$ & $3 \%$ & $3 \%$ \\
\hline Nuclear & $4 \%$ & $4 \%$ & $4 \%$ \\
\hline Hydro/renewables & $14 \%$ & $13 \%$ & $12 \%$ \\
\hline Generation (TWh) & 3,394 & 4,368 & 5,358 \\
\hline \multicolumn{4}{|l|}{ Conservative } \\
\hline$\overline{\text { Coal }}$ & $81 \%$ & $83 \%$ & $84 \%$ \\
\hline Gas & $2 \%$ & $2 \%$ & $2 \%$ \\
\hline Nuclear & $3 \%$ & $3 \%$ & $3 \%$ \\
\hline Hydro/renewables & $14 \%$ & $12 \%$ & $11 \%$ \\
\hline Generation (TWh) & 3,238 & 3,961 & 4,627 \\
\hline \multicolumn{4}{|l|}{ Optimistic } \\
\hline Coal & $79 \%$ & $75 \%$ & $74 \%$ \\
\hline Gas & $2 \%$ & $6 \%$ & $8 \%$ \\
\hline Nuclear & $4 \%$ & $4 \%$ & $4 \%$ \\
\hline Hydro/renewables & $15 \%$ & $15 \%$ & $14 \%$ \\
\hline Generation (TWh) & 3,551 & 4,780 & 6,151 \\
\hline
\end{tabular}

By 2020 , the total generation of electricity varies by about $1,500 \mathrm{TWh}(+15 /-15 \%$ from $\mathrm{BAU}$ ) under these three scenarios. Following the assumptions used for price and availability of primary fuels, the price and efficiency characteristics of available technology, and the host of central development and environmental initiatives associated with the electricity sector, coal-fired electricity still dominates, although its share is lessoned under the optimistic, but slightly increased under the conservative scenario. Under BAU conditions, the structure of electricity generated is not expected to change much into the future. 
The most important difference amongst these scenarios to note occurs under the optimistic scenario where the price and availability of natural gas favors comparatively to coal. Here, these developments result in the proportion of electricity generated from gas rising much more quickly than in the other two scenarios. Under each scenario, nuclear generation contributes 3 to $4 \%$ of total electricity generated, in line with central initiatives. Hydro and renewables remain constant or fall in proportion due largely to the lower prominence of large scale hydro-electric projects into the future.

Emission intensity by region by scenario

Key for CDM baseline analysis are changes in the emission intensity of electricity provided to end users over time as this forms an essential component of calculating the emission reductions achievable by proposed CDM projects (see figures $20,21,22$ ). 
Figure 20. Emission intensity by region as calculated by the IPM - BAU scenario

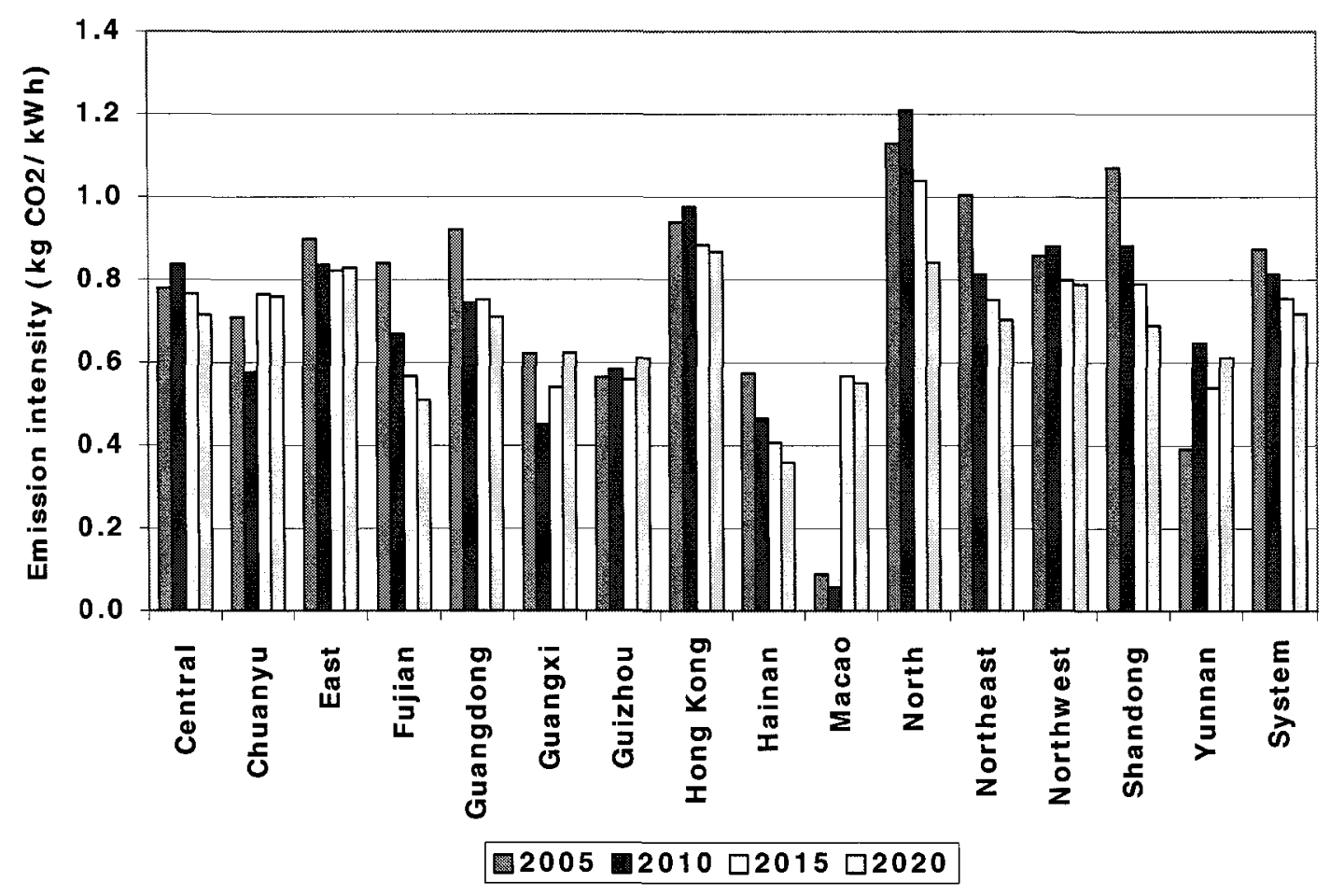

Figure 21. Emission intensity by region as calculated by the IPM - Optimistic scenario

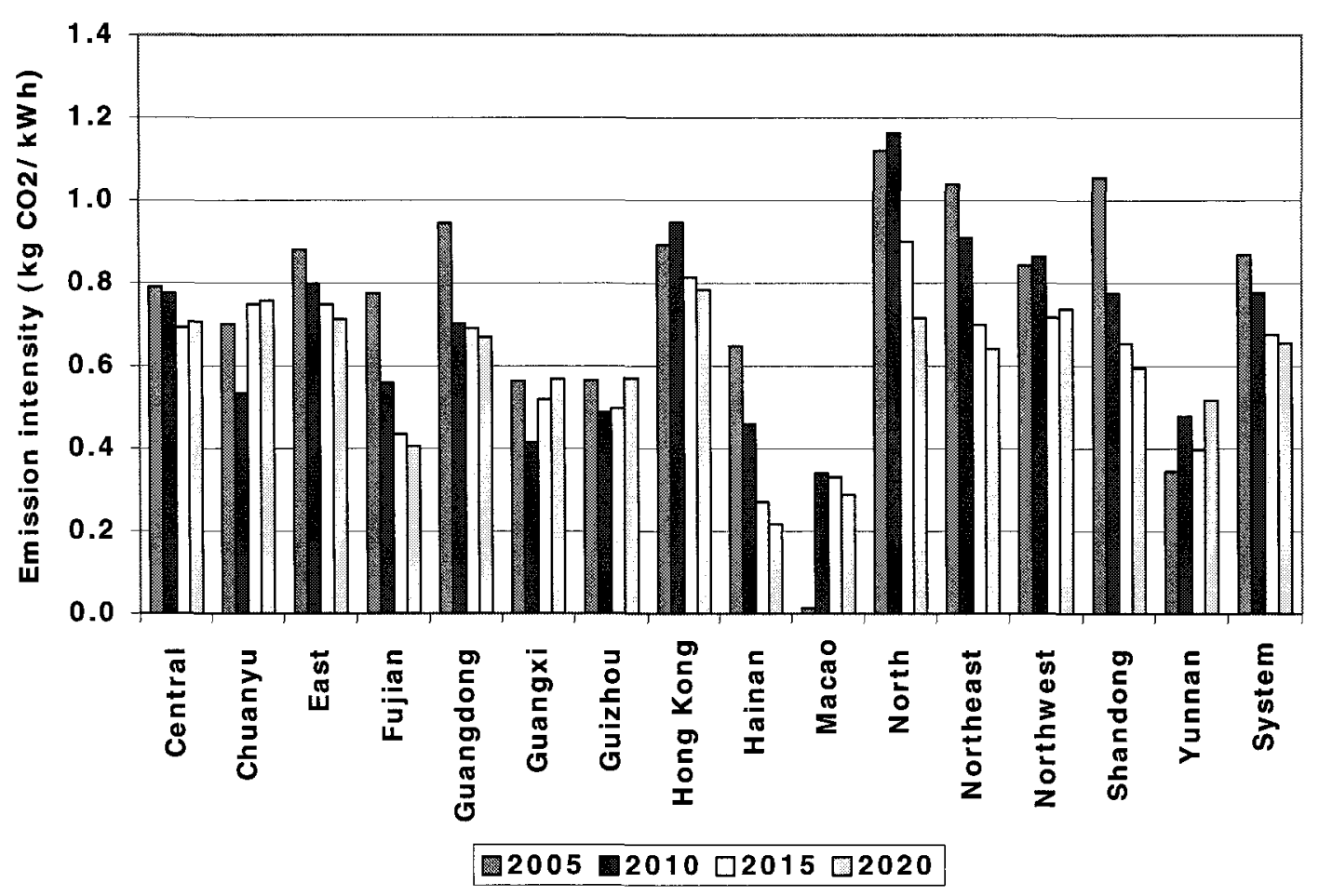


Figure 22. Emission intensity by region as calculated by the IPM - Conservative scenario

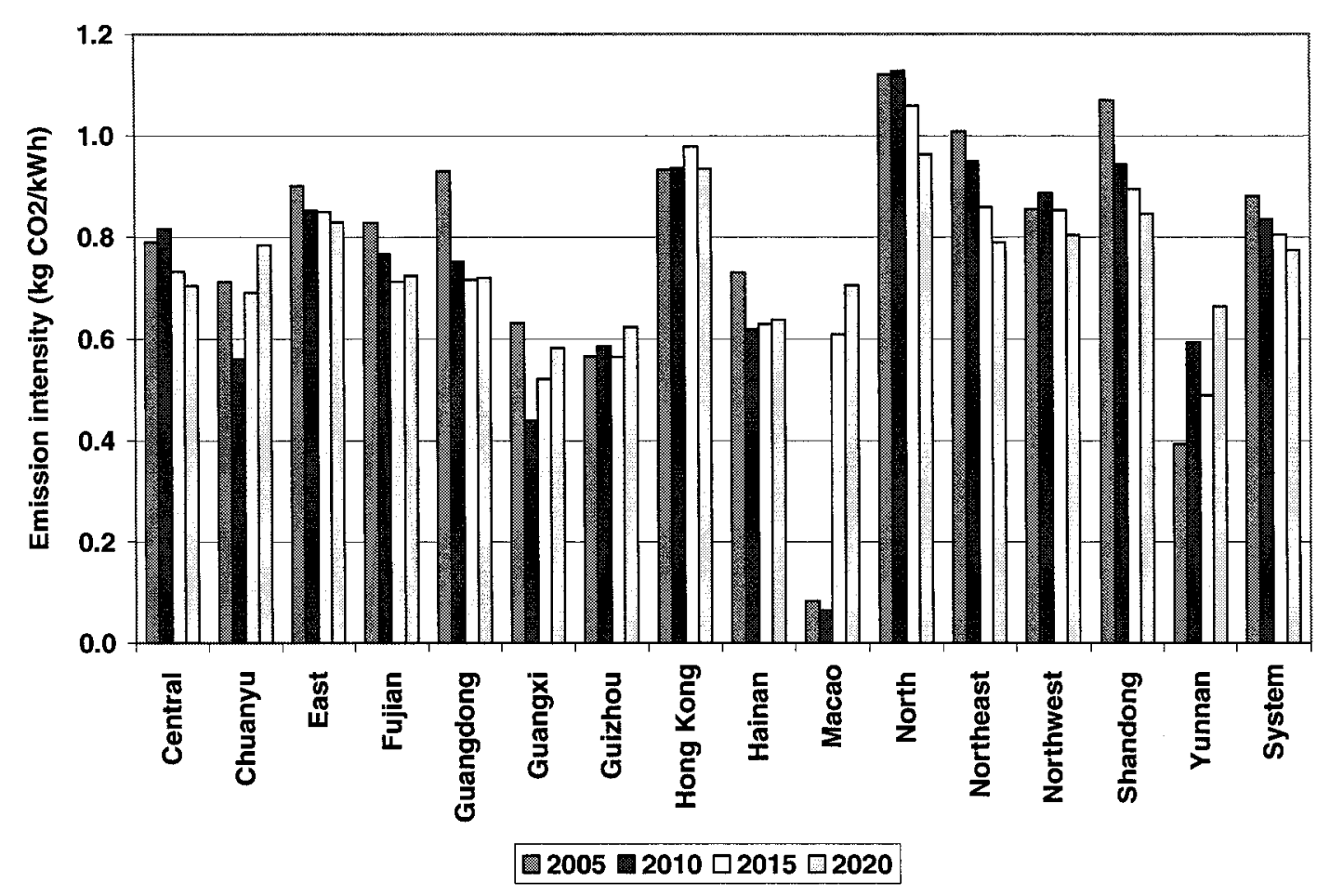

Under the BAU scenario, the system wide emission intensity for electricity decreases nearly $18 \%$ between 2005 and 2020 . This falls within the range of emission intensity reduction that was achieved using the decomposition approach for emission baseline calculation. However, there is no regional consistency in the change in emission intensity. In some regions (such as the central region and China's north) the emission intensity rises in 2010 , before declining thereafter. The explanation for this is the long lead times in these regions to increase the role of natural gas, or in some cases nuclear. In other regions such as Fujian, Guangdong, or China's northeast (including Beijing), the emission intensity falls much more rapidly under BAU conditions. The primary reason for this are the more rapid increases seen in both natural gas fired electricity generation and/or the building of nuclear generating capacity in these regions. 
The emission intensity falls much more under the optimistic scenario than compared to the BAU, decreasing nearly $25 \%$ between 2005 and 2020 . As was explained previously, the majority of this was due to a greater proportion of electricity being generated by natural gas and nuclear, although this scenario also includes faster penetration of CCT and therefore a faster increase in energy efficiency. The standard deviation of the regional baselines is much higher than the BAU, for example. This indicates the exaggerated regional differences that emerge due to alternative developments in factors such as natural gas accessibility and the speed of technological development. Alternatively, the emission intensity in the conservative scenario is consistently higher than the other two scenarios for each region considered. This reflects largely the increased share of coal-fired electricity in each region within this scenario.

\subsection{Chapter conclusions}

Two main conclusions are drawn from the scenario analysis completed above; the methodological and the substantive lessons learned.

Methodologically, two techniques have been used to construct three scenarios following three similar storylines but although following the same storyline and both forward looking scenario generation techniques, each is still fundamentally different in its approach to generating the scenarios. Factor decomposition was used to first quantify the different factors that contribute to a change in emissions or emission intensity. Using the storylines generated, these same factors were then studied in terms of how they might change under each scenario storyline, with these changes then used to calculate a change in emissions or emission intensity. Alternatively, dispatch modelling is a simulation approach where the electrical sector organizes in dynamic response to a number of 
drivers that change over time. Comparing these two approaches, it is evident that the decomposition approach is at a disadvantage due to its inability to consider regional details and its inability to properly account for economic decision making in the sector, particularly in terms of the impacts of possible changes in the price of coal relative to natural gas.

Substantially, a number of things can be concluded from the above scenario analysis. Under the BAU scenario, the average emission intensity of electricity generation in China is gradually expected to decrease with time, even if total emissions increase. Similar to the past, a significant contributor will be the efficiency of electricity generation. Increases in natural gas-fired generation (in a response to efforts to increase the accessibility to this fuel source) and increases in electricity generated from nuclear sources will also contribute significantly to a reduction in emission intensities. Nonetheless, there are significant uncertainties that exist on the contribution of natural gas, nuclear and renewables to the electricity mix, with this largely determined by the financial capabilities within the country, the ability to build supply infrastructure and capital projects quickly, as well as political decisions and cooperation between local/regional governments and the central government. 


\section{Operationalizing the baselines}

Synopsis: The previous chapters reviewed the methodological attributes underlying baseline and scenario development and then developed these baselines analytically. In this chapter these baselines are now operationalized by applying these to a series of hypothetical CDM case studies. This shows the projected emission reductions achieved under different baseline approaches and helps to illustrate the strengths and weaknesses of any one baseline method across the entirety of the baseline analysis process.

\subsection{Chapter introduction}

The baseline methods studied and developed analytically in previous chapters are put into operation by applying these to a series of hypothetical CDM projects that could be implemented into China's electrical sector. Each hypothetical project is placed in the context of the relevant policy and economic environments in China, thereby ensuring these would meet the additionality requirements for CDM projects. Establishing CDM project examples allows emission reductions to be calculated for each baseline method and project considered, in turn enabling an understanding to be gained of how the different baseline approaches used impact upon the assessment of additionality. Using the criteria given for assessing baseline methods (see chapter 3), a review and assessment is made across the entirety of the baseline analysis process (i.e. from the initial additionality assessment, collection of data, establishing of the BAU and alternative scenarios, to the calculation of the baseline and measurement of project additionality). 


\subsection{Establishing hypothetical CDM projects}

In order to illustrate how the selection of a baseline method effects the assessment of additionality, hypothetical CDM projects suitable for China's electrical sector are developed. Five different technologies are included (see table 20 for technical details):

1) Combined cycle natural gas-fired electricity generator fueled by coal mine methane (CMM)

2) Wind farm comprised of multiple wind turbines (WG)

3) Super-critical, coal-fired generation power plant (clean coal technology, or CCT)

4) Combined cycle (tri-generation) natural gas peaking plant (NG)

5) Small hydro electricity generation $(\mathrm{SH})$

Table 20. Technical details of hypothetical CDM case studies

\begin{tabular}{|c|c|c|c|c|c|}
\hline $\begin{array}{l}\text { Project case } \\
\text { study }\end{array}$ & $\frac{\text { Coal mine }}{\underline{\text { methane }}}$ & $\frac{\text { Wind }}{\text { farm }}$ & $\frac{\text { Clean coal }}{\underline{\text { technology }}}$ & $\frac{\frac{\text { Combined }}{\text { cycle natural }}}{\text { gas }}$ & $\frac{\begin{array}{c}\text { Small-scale } \\
\text { hydro electric }\end{array}}{\underline{\text { station }}}$ \\
\hline Fuel & Natural gas & Wind & Coal & Natural gas & Hydro \\
\hline Capacity (MW) & 120 & 50 & 3,600 & 250 & 200 \\
\hline $\begin{array}{l}\text { Capacity factor } \\
(\%)\end{array}$ & 0.4 & 0.3 & 0.8 & 0.4 & 0.35 \\
\hline Hours per year & 3,504 & 2,628 & 7,008 & 3,504 & 3,066 \\
\hline Efficiency (\%) & 0.44 & $\mathrm{NA}$ & 0.38 & 0.38 & NA \\
\hline $\begin{array}{l}\text { Annual } \\
\text { generation (GWh) }\end{array}$ & 420 & 131 & 25,229 & 876 & 613 \\
\hline $\begin{array}{l}\text { Annual project } \\
\mathrm{CO}_{2} \text { emissions } \\
\text { (tonnes) }\end{array}$ & 165,134 & 0 & $20,076,813$ & 398,349 & 0 \\
\hline $\begin{array}{l}\text { Project emission } \\
\text { intensity } \\
(\mathbf{k g C O} / \mathrm{KWh})\end{array}$ & 0.393 & 0.000 & 0.796 & 0.455 & 0.000 \\
\hline
\end{tabular}




\subsubsection{Geographical distribution of CDM project case studies}

Each technology type is applied in multiple geographical regions in China in order to test the geographical applicability of each baseline method (see figure 23). In total, this means that 23 individual CDM projects will be assessed: $5 \mathrm{CMM}$ projects, $5 \mathrm{CCT}$ projects, 5 natural gas projects, 4 wind generation projects, and 4 small hydro projects. The distribution of the hypothetical CDM projects follows the resource endowments and economic circumstances of each region in China.

\section{Figure 23. Geographical location of CDM project case studies}

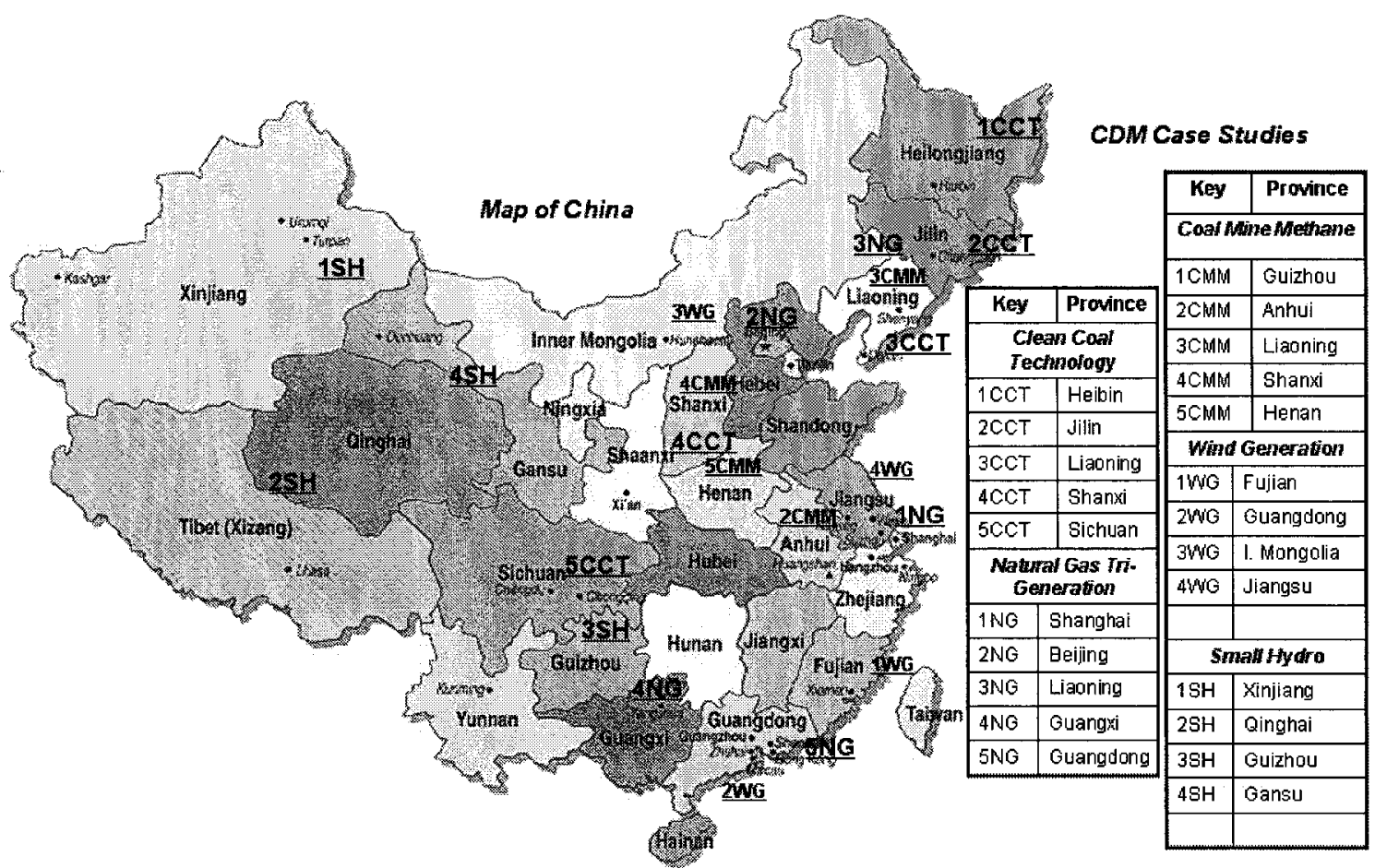


The key reasons underlying the geographical location of each project case study (and the main sources used for these purposes) include:

- Clean coal technology (CCT) project case studies were placed in coal-dominated grids;

- Natural gas (NG) project case studies were placed in the proximity of larger urban centres as it is only in highly populated areas where the tri-generation of electricity, heating and cooling capabilities of these projects can be fully utilized;

- Coal mine methane (CMM) project case studies were placed at mines that produce large amounts of methane per unit of coal extracted. This knowledge was gained from feasibility studies of potential CMM projects at different coal mines in China completed by the China Coalbed Methane Clearinghouse (CCBMC, 2001c; a; b; d);

- Wind generation (WG) project case studies were placed in areas with wind resources above thresholds required for the production of electricity from wind. The specific location of each case study was gained by examination of regional wind maps for China (Elliott et al., 2002), and;

- Small hydro (SH) project case studies were placed in the southwestern and western reaches of China where there are sufficient water resources, and also areas considered rural and the target of related rural electrification policies. 


\subsubsection{Project type additionality and national circumstance}

The additionality of each project type was determined by considering the national circumstance of each and how the project type fared when assessed according to the Additionality Tool (Rigius, 2004) (see table 21). Details are contained in Appendix A. 
Table 21. Summary of project type national circumstance and additionality

\begin{tabular}{|c|c|c|}
\hline$\frac{\text { Project }}{\text { type }}$ & $\frac{\text { Mains points of national }}{\text { circumstance }}$ & Main points of additionality \\
\hline $\begin{array}{l}\text { Clean coal } \\
\text { technology }\end{array}$ & $\begin{array}{l}\text { Not widely employed (i.e. } \\
\text { less than } 10 \mathrm{GW} \text { as of } \\
2002 \text { ) } \\
\text { No comprehensive } \\
\text { policies to promote clean } \\
\text { coal technology }\end{array}$ & $\begin{array}{l}\text { - Not widely employed } \\
\text { - CER sales would directly contribute to } \\
\text { project financial viability when } \\
\text { considering the large emission } \\
\text { reduction potential of CCT projects }\end{array}$ \\
\hline $\begin{array}{l}\text { Tri- } \\
\text { generation } \\
\text { combined } \\
\text { cycle } \\
\text { natural } \\
\text { gas }\end{array}$ & $\begin{array}{l}\text { Natural gas infrastructure } \\
\text { is being rapidly } \\
\text { constructed (including } \\
\text { both pipelines and LNG } \\
\text { terminals), but there are } \\
\text { limited primary natural } \\
\text { gas resources in China }\end{array}$ & $\begin{array}{l}\text { - Natural gas contributes only minor } \\
\text { amounts }(2 \%) \text { to national grid, } \\
\text { There are no tri-generation projects in } \\
\text { China due to cost barriers and lack of } \\
\text { domestic technological capacity } \\
\text { - Technology has multiple benefits due } \\
\text { to cooling, heating and electricity } \\
\text { provided } \\
\text { CER sales will contribute significantly } \\
\text { to this project type's financial viability } \\
\text { due to high operational costs of natural } \\
\text { gas-fired electricity }\end{array}$ \\
\hline $\begin{array}{l}\text { Coal mine } \\
\text { methane }\end{array}$ & $\begin{array}{l}\text { Approximately } 12 \mathrm{BCM} \\
\text { of methane released into } \\
\text { atmosphere annually from } \\
\text { China's coal mines } \\
\text { No requirements for the } \\
\text { capture of the methane }\end{array}$ & $\begin{array}{l}\text { Few schemes to use CMM for } \\
\text { electricity generation } \\
\text { CERs from CMM projects would } \\
\text { greatly contribute to the financial } \\
\text { viability of a project } \\
\text { - Capture of CMM greatly improves } \\
\text { mine safety }\end{array}$ \\
\hline Wind & $\begin{array}{l}\text { China has enormous wind } \\
\text { generation potential } \\
\text { Most of the wind } \\
\text { generation capacity in } \\
\text { China is small sized, and } \\
\text { the country lacks } \\
\text { domestic capability to } \\
\text { produce large scale wind } \\
\text { farms. }\end{array}$ & $\begin{array}{l}\text { China has a significant lack of domestic } \\
\text { capacity to design, manufacture and } \\
\text { implement large scale wind farms. }\end{array}$ \\
\hline $\begin{array}{l}\text { Small } \\
\text { hydro }\end{array}$ & $\begin{array}{l}\text { China has enormous } \\
\text { hydro resources: } 17.6 \% \text { of } \\
\text { global total } \\
\text { - Small hydro prominent in } \\
\text { many of China's } \\
\text { mountainous regions } \\
\end{array}$ & $\begin{array}{l}\text { - Small hydro still heavily supported by } \\
\text { government loans } \\
\text { - Small hydro has far-reaching } \\
\text { sustainable development attributes }\end{array}$ \\
\hline
\end{tabular}




\subsection{Application of baselines}

The results of the different baseline analyses are used to establish the environmental additionality of the hypothetical CDM projects by comparing the project emission intensity and the BAU baseline emission intensity for 2010 and 2020 (see table 22). Since this is considered to be the most accurate method by the UNFCCC, the baselines calculated using the dispatch model (DM) are then used to determine the cumulated emission reductions that might be expected over the first reporting period (2008 to 2012). Following the suggestions of the CDM EB, the operating margin baseline is applied only to projects that are intermittent or that affect mainly peak-load (i.e. wind and small hydro) while build margin baselines are applied to projects that could act as base-load (i.e. CCT, $\mathrm{NG}$, and $\mathrm{CMM}$ ). 
Table 22. Calculated GHG baselines ( $\mathrm{kg} \mathrm{CO} / \mathrm{KWh}$ ) and emission reductions (ktonnes $\mathrm{CO}_{2}$ ) by baseline method and $\mathrm{CDM}$ project case study

'DA' = dispatch analysis 'OM' = operating margin 'BM' = build margin 'DM' = dispatch model. Shaded and stroked out cells indicate projects which do not remain environmentally additional, with projects determined to be non-additional based upon the dispatch modelling approach bolded for emphasis (as this is deemed the most accurate method). An N/A has been inserted where the baseline method is not appropriate for the project types being analyzed.

\begin{tabular}{|c|c|c|c|c|c|c|c|c|c|c|c|c|}
\hline \multirow{2}{*}{$\begin{array}{c}\text { Project } \\
\text { ID }\end{array}$} & \multirow[b]{2}{*}{ Province } & \multirow[b]{2}{*}{ Grid } & \multirow{2}{*}{$\begin{array}{c}\text { Project } \\
\text { emission } \\
\text { intensity } \\
(\mathbf{k g} \\
\text { co2/KWh) }\end{array}$} & \multicolumn{4}{|c|}{$\mid \begin{array}{c}\text { Emission baseline in } \\
2010(\mathrm{~kg} \mathrm{CO} / \mathrm{KWh})\end{array}$} & \multirow{2}{*}{\begin{tabular}{|c|} 
Emission \\
reductions \\
possible \\
(ktonnes) \\
$(2008-2012)$ \\
\end{tabular}} & \multicolumn{4}{|c|}{$\begin{array}{l}\text { Emission baseline in } \\
2020(\mathrm{~kg} \mathrm{CO} 2 / \mathrm{KWh})\end{array}$} \\
\hline & & & & DA & OM & BM & DM & & DA & OM & $\mathbf{B M}$ & DM \\
\hline$\overline{C C T}-1$ & Heelibin & Northeast & 0.80 & 0.86 & N/A & 0.86 & 0.81 & 2,200 & 0.77 & $\mathrm{~N} / \mathrm{A}$ & 0.86 & 8.78 \\
\hline CCT - 2 & Jalin & Northeast & 0.80 & 0.86 & /A & 0.86 & 0.81 & 2,200 & 9.77 & N/A & 0.86 & 0.78 \\
\hline CCT - 3 & Liaoning & Northeast & 0.80 & 0.86 & N/A & 0.84 & 0.81 & 2,200 & 0.77 & $\mathrm{~N} / \mathrm{A}$ & 0.84 & 0.70 \\
\hline CCT - 4 & Shanxi & North & 0.80 & 0.86 & N/A & 0.85 & 1.21 & 52,183 & 0.77 & $\mathrm{~N} / \mathrm{A}$ & 0.85 & 0,1 \\
\hline CCT -5 & Sichuan & Chuan & 0.80 & 0.86 & N/A & 0.98 & 8.50 & 27,648 & 0.7 & /A & 0.98 & 0,70 \\
\hline & Shar & $\mathrm{Ea}$ & 0.45 & 0.86 & $N$ & 0.48 & 0.84 & 1,669 & 0.77 & $N / A$ & 0.48 & 0.83 \\
\hline & Beij & & 0.45 & & N/ & & 1.21 & 3,306 & 0.77 & $N / A$ & 0.50 & 0.84 \\
\hline & & & 0.45 & & & & 0.81 & 1,570 & 0.77 & & .84 & 0.70 \\
\hline & & & 0.45 & & & & 0.45 & 20 & 0.77 & & 20 & 0.62 \\
\hline 5 & Guar & Guangdong & 0.45 & 0.86 & $\mathrm{~N}$ & 0.68 & 0.74 & 1,266 & 0.77 & $N / A$ & 0.68 & 0.71 \\
\hline-1 & & & 0.00 & 0 & & $\mathrm{~N} /$ & 0.67 & 439 & 0.77 & 0.82 & N/A & 0.51 \\
\hline & Gua & ggdong & 0.00 & 0.86 & & N/A & 0.74 & 489 & 0.77 & 0.81 & N/A & 0.71 \\
\hline-3 & I. Mon & No & 0.00 & 0.86 & 0.87 & N/A & 1.21 & 795 & 0.77 & 0.87 & $N / A$ & 0.84 \\
\hline WG - 4 & Juar & & 0.00 & 0.86 & & N/A & 0.84 & 549 & 0.77 & 0.84 & $N / A$ & 0.83 \\
\hline & & & 0.00 & & & N/A & 1.01 & 3,097 & 0.77 & 1.01 & $N / A$ & 1.01 \\
\hline $\mathrm{SH}-2$ & & & 0.00 & 0.86 & 0.99 & N/A & 0.88 & 2,701 & 0.77 & 0.99 & $\mathrm{~N} / \mathrm{A}$ & 0.79 \\
\hline & & & 0.00 & 0.86 & 0.85 & N/A & 0.88 & 2,706 & 0.77 & 0.85 & N/A & 0.61 \\
\hline $\mathrm{SH}-4$ & & & 0.00 & 0.86 & 0.83 & $N / A$ & 0.88 & 2,701 & 0.77 & 0.83 & N/A & 0.79 \\
\hline CMM - 1 & & & 0.39 & 0.86 & N/A & 0.00 & 0.58 & 402 & 0.77 & $\mathrm{~N} / \mathrm{A}$ & 0.00 & 0.61 \\
\hline CMM - 2 & & & 0.39 & & $N / A$ & 0.82 & 0.84 & 934 & 0.77 & N/A & 0.82 & 0.71 \\
\hline CMM - 3 & Lianoning & Northeast & 0.39 & 0.86 & $N / A$ & 0.84 & 0.81 & 884 & 0.77 & $N / A$ & 0.84 & 0.70 \\
\hline CMM - 4 & & & 0.39 & 0.86 & $\mathrm{~N} / \mathrm{A}$ & 0.85 & 1.21 & 1,717 & 0.77 & $N / A$ & 0.85 & 0.84 \\
\hline CMM - 5 & Henan & Chuanyu & 0.39 & 0.86 & N/A & 0.00 & 0.58 & 387 & 0.77 & $\mathrm{~N} / \mathrm{A}$ & 0.00 & 0.76 \\
\hline
\end{tabular}

A number of key points are noted concerning the temporal progression of the applicability of technology for certified emission reductions under the various baselines, the geographical potential of CDM projects under each baseline method, and the 
variation across the different baseline methods in terms of the baseline emission factor proposed.

\subsubsection{Temporal progression of CDM project eligibility}

A number of projects are not environmentally additional according to the dispatch method in 2010 (the mid-point of the first reporting period of the Kyoto Protocol), including a combined cycle natural gas plant in Guanxi (a grid dominated by hydro electric resources) and a clean coal technology plant in Sichuan (also a grid impacted by the Three Gorges and other hydro electricity projects). By 2020, a number of other technologies are further removed from being a certifiable CDM project. In China's northwest, clean coal technology becomes non-additional according to both the decomposition analysis (DA) and the dispatch method (DM). This reflects the increasing contributions of nuclear, natural gas, and to a certain degree CCT that is assumed to take place in the BAU scenario generated using these methods.

\subsubsection{Geographical potential of CDM projects}

The opportunities for certifiable CDM projects are concentrated in regions that are dominated by coal-fired electricity, including in China's east, northeast, and north. For example, the project with the largest emission reduction potential is situated in Shanxi province, located in China's heavily industrialized north. Here, the base-load CCT project could achieve over $52 \mathrm{MTCO}_{2}$ of emission reductions by 2020 , significant when considering that this is a coal-fired generation unit and not a zero emission source, such as for wind or small hydro. Regions dominated by hydro resources, such as China's 
south-central region, have far less opportunity for emission reduction due to the low GHG-intensive electricity sources within these regions.

\subsubsection{Variation across baseline method}

In 2010 the average difference across the different methods is only $6 \%$, but with time this increases and by 2020 the average difference exceeds $40 \%$. This variation is largely explained by the difference between the baseline results achieved using the dispatch method and those using the build/operating margin method as the first of these is dynamic while the latter are not. A direct result of this difference appears under the BAU assessment where the dispatch method assumes increasing levels of efficiency and increasing levels of natural gas and nuclear and thus the baseline falls relative to the static operating and build margin approach.

\subsection{Review and appraisal}

The relative strengths and weaknesses of each baseline analyses are assessed by considering the criteria presented in table 5 of chapter 3 . These criteria included the following:

Baselines must:

1) Ensure environmental additionality;

2) Provide rigour and accuracy to ensure that net reductions in anthropogenic emissions are real and measurable;

3) Ensure geographical applicability and properly account for spatial scale; 
4) Account for applicable national policies and specific national or regional circumstances, such as sectoral reform initiatives, local fuel availability, power sector expansion plans, and the economic situation in the sector relevant to the project activity;

5) Be transparent and transferable with regard to the choice of assumptions, parameters, and data sources, and;

6) Take account of uncertainties by using conservative assumptions.

\subsubsection{Environmental additionality}

In order to meet the overarching environmental goals of the Kyoto Protocol, it is critical that the environmental additionality of a CDM project be achieved. There are three individual criteria that contribute significantly to this overarching objective: the rigour and accuracy of the baseline; its geographical applicability; and the ability to account for national and regional circumstances. Due to the detailed structure of the model, the dispatch model is best able to meet each one of these criterion, and therefore qualitatively it can be concluded this is the best available technique for ensuring environmental additionality. Further, it is noted that the choice of the baseline method has a significant impact on the assessment of additionality. For grids that experience more rapid change in terms of generation mix or fuel shares, wide variations in baseline values emerge when using different baseline methods.

\subsubsection{Rigour and accuracy}

Rigour and accuracy refer to the realism of the baseline method. There will likely be significant future changes in China's electrical sector that will affect emissions. 
Therefore, rationally the operating/build margin baseline methods fail to meet this criterion since these are static in nature. Alternatively, dispatch modelling is more rigorous because its forward-looking framework includes drivers which can interact so that the electrical system evolves to reflect the optimal organization of these drivers. The dispatch modelling approach is also able to consider regional variations within these specific drivers, unlike decomposition analysis. Because most electrical sectors across the world are undergoing or have undergone processes of liberalization, dynamics based upon economic optimization is more realistic than a static type of analysis, such as contained within the operating/build margin approaches or the decomposition analysis.

\subsubsection{Geographical applicability}

Geographical applicability refers to how or if a baseline is able to consider regional differences of factors affecting the environmental quality of electricity generated (e.g. generation source mix, fuel shares, technology characteristics). Decomposition analysis can not distinguish between different geographical regions since this can only be calculated at the national level. Operating and build margins do reflect geographical differences but likewise this can be severely limited by data availability. In China, data on the regional structure of the electricity sector is available only for the most recent years, with little information available on the transfers of electricity between regions. So it is only dispatch models which are able to properly consider geographical scale since these are able to a) represent the geographical variability of the electrical sector in terms of electricity generated, b) adequately include the effect of electricity transfers, and c) determine the temporal development of both of these components. 


\subsubsection{National/regional circumstance}

When undertaking futures analysis it is very important to understand the political, economic, social, and technological development of any region or nation since these factors could have significant impacts on the emission intensity of electricity provided to end users. A baseline method must therefore be able to embrace these changes in its methodological framework.

The operating and build margins do give an indication of these circumstances for the present period but since economic circumstances are changing incredibly fast in China, such analysis of the electrical sector risks quickly becoming outdated. Even though technology is not advancing at the speed of capital construction, the structure of the electricity generating sector has the ability to change rapidly in terms of the contribution of the different types of generation to the total source mix.

Alternatively, dispatch analysis can better consider unique and changing national circumstances in its analytical framework if the expert knowledge that feeds into the establishment of the drivers included properly account for these factors. Alternative scenarios can further this by helping to illustrate the potential impacts of changes in any of the driving factors. To a lesser degree, decomposition analysis can also account for changing national and regional circumstance if properly accounted for in the factors decomposed.

\subsubsection{Transparency and transferability}

The transparency and transferability of a baseline method with regards to the choice of assumptions, parameters, and data sources included is important because a transparent 
method is easier to evaluate than a 'black box' model and will also be more readily reproducible.

Of the methods pursued, the operating and build margins are the most transparent and easy to use. Although there are variations in how these baselines are calculated, the general approach is simple to understand and the algorithms simple to apply. The data is readily available, there are no complex interactions or formula, and the outputs are easy to analyze and communicate.

The dispatch modelling approach is much less transparent. The algorithms this relies upon are endogenous and usually can only be manipulated by programmers who do not necessarily have expertise in the operation of the electrical sector. Therefore, unusual results can arise that might not seem logical to users unless a more sophisticated understanding is gained of the inner workings of the model. The objective function of the model may be simple to communicate, but it includes a series of interacting variables. Dispatch modelling requires very detailed information not only at the regional level, but also on future electricity demand, generation source mix, the types of generation technologies used, the respective cost and efficiency characteristics of generation technologies, fuels, and the load profile in terms of electricity demand. The values for each of these must be assured in order to increase the accuracy of the results. This decreases the transparency of the dispatch modelling approach, both because of the 'black box' characteristics of the model and because experts are needed to use it. Finally, the simplicity of the decomposition approach, the easily understood nature of the decomposition equations, and macro nature of the approach, increases its transparency to 
the user. Arguably, users are more apt to understand the big picture that decomposition analysis provides.

\subsubsection{Data availability}

The availability and accessibility of data is critical for the transparency and transferability of a baseline, particularly the amount of data that is required and the knowledge required in order to utilize the data. This deserves a closer examination as the practicalities of the data requirements change with each method and for each country that is being analyzed.

The decomposition approach is very data intensive as it requires a long time series of historical data, data that is typically only available on a national basis unless large amounts of money are expended. Even though the method is relatively data intensive, however, the relationships formed are relatively straightforward to determine and analyze. Nonetheless, especially in China there are uncertainties on the accuracy of data used for a number of important parameters contained within the decomposition analysis, particularly concerning historical data on economic output and energy demand. Numerous authors have called into question the reliability and accuracy of China's economic and energy statistics over long periods of time (see Sinton, 2005 and the references within).

Alternatively, the operating and build margins require detailed data at the regional level. In China, this information is readily available from national statistical sources, but only for the most recent years. Dispatch modelling also requires very detailed information at the regional level, but in addition also requires information on future occurrences. Therefore, the dispatch modelling approach is not only very data intensive but also knowledge intensive as well. For example, in order to be able to model an 
electrical system using dispatch modelling, the current configuration of the sector must be first understood at a very detailed level, including the generation source mix, the types of generation technologies that are used, the respective cost and efficiency characteristics

of generation technologies, and the load profile in terms of electricity demand. Further to this, in order to determine the optimized structure of electricity generation in future years, values must be established for the set or drivers contained within the model, including future changes in technology (including parameters such as efficiency and cost), how fuel markets might develop, and how the load profile of electricity demand might evolve in terms of peak-load versus base-load. This all means that a detailed study of the demand sector must be undertaken, including ultimately, a detailed understanding of the regional economies that exist in China and how these might evolve into the future due to both external and internal economic, political and technological dynamics.

\subsubsection{Uncertainty}

Uncertainty is a function of the speed of change and the variability of this change. It is also a function of time. In a dynamic system such as the electrical sector, uncertainty increases with time. Since the operating and build margins are static, they can not properly account for uncertainty that increases in the temporal dimension.

Decomposition analysis is limited to the factors that have been included in the analysis. There is uncertainty about the importance of the factors studied and about the linearity of the analytical technique. Dispatch modelling, alternatively, includes many different variables, each important in determining the objective one tries to reach. The inclusion of drivers that can change with time, and the inclusion of a framework which 
responds to these drivers in a dynamic way increases the ability of this method to handle uncertainty. This is especially the case if conservative assumptions are used. 


\section{Summary and conclusions}

\subsection{The research context and purpose}

The global carbon market is rapidly developing as the first commitment period of the Kyoto Protocol draws closer and Parties to the Protocol with emission reduction targets seek alternative ways to reduce their GHG emissions. The Protocol includes three flexibility mechanisms that allow Parties to purchase emission reductions from the international market or make project-based investments in non-Annex I nations that will lead to additional reductions in GHG emissions. The CDM, one of these three mechanisms, allows a legal entity from an Annex I nation or a non-Annex I nation to make a project-based investment in the non-Annex I nation. The emission reductions that result from this project can then be transferred to the originating nation in order to help them reach their domestic emission targets or can be sold to the international market.

China is drawing considerable CDM interest given its size, the technological characteristics of its economy and the country's reliance upon coal for most of its energy needs. As China's economy modernizes, more technologies and processes are requiring electricity and demand for this energy source is accelerating rapidly. Rapid economic growth coupled with relatively inefficient technology to generate electricity in China makes the electrical sector a major source for potential GHG emission reduction opportunities as related to the CDM.

The implementation of the CDM within China and other developing countries is a complicated and often expensive process due to high transaction costs, institutional barriers, and political and economic uncertainty. In particular, the proper assessment of 
the environmental additionality of $\mathrm{CDM}$ projects continues to be a challenge for the CDM project investor and for the broader climate policy community. It is this latter area where the core of the analysis completed within this dissertation is most applicable. The research indirectly illustrates that the implementation of the CDM will continue to be hampered by substantial costs. In addition to the administrative costs concerning registering the CDM project and the costs associated with developing the needed institutional frameworks within the developing country, the analytical costs associated with establishing an emission baseline and the environmental additionality of the CDM project are significant. In terms of the different baseline methods studied, the DM can provide the most accurate estimate of future additionality in the electrical sector, but this method requires considerable technical expertise and extensive data that is expensive to collect and difficult to verify without substantial research.

Assessments of the environmental additionality of the CDM are important for at least two reasons. The first is that the baseline analysis used in the CDM approval process must be scientifically sound, credible, and accessible for both others to use and for evaluation purposes. The second reason relates to the long-term accuracy of the environmental additionality estimates and hence the prospects for meeting the overall CDM goals of reducing GHG emissions globally. For example, systematic overestimation of the emission baseline could result in considerably less than anticipated environmental additionality over the CDM lifespan.

This research investigates three different baseline methodologies available for GHG emission baseline construction and operationalizes these in the context of China's electrical sector with the purpose to understand how or if these methods meet these 
general criteria. This includes collecting and compiling data required to implement each methodology, constructing the baselines, setting three plausible scenarios for the future emission levels under each of these scenarios, and then measuring a series of hypothetical CDM project case studies against these baselines. The baseline methodologies studied include factor decomposition, build and operating margins, and dispatch modelling. Each method varies according to their analytical perspective and in terms of the techniques utilized: factor decomposition is based upon a historical analysis and relies upon the disaggregation of trends, build and operating margins are based on the current technological and economic characteristics of the electrical sector and rely on detailed statistics of the sector, while dispatch modelling is based upon simulation analysis and requires detailed data in conjunction with a simulation model.

Operationalizing the different baseline methods provided a foundation for gaining insight into fundamental issues underpinning CDM projects as well as enhancing understanding of the role of China's electrical sector as an integral component of both its own domestic economic growth and its role in the global carbon market.

\subsection{Primary findings}

Five research questions were posed in Chapter 1. Each question focused on a specific aspect of China's development or CDM methodologies, and collectively provide insight into prospects for the long-term development of China's electrical sector, the role that CDMs might play in this development process, and issues relating to baseline methodology. 
Question 1: What is China's strategic importance generally and in particular China's electrical sector in the global climate change regime?

China currently plays and will increasingly play an important role within the global carbon market as both a GHG emitter and potential source for emission reductions. The electrical sector is positioned to attract a large proportion of the total supply of CDM projects within China.

The reasons lie in China's rapid economic growth, the high energy and carbon intensity of its economy, and the role and characteristics of the electrical sector within the economy. On a sectoral basis, the electrical sector is the most important in China for both emissions and emission reduction possibilities because electricity consumption is growing rapidly and because much the technology used to generate electricity is not as efficient as the best practices available (as promoted by the CDM). Thus, there is a strong argument for a focus on China's electrical sector.

Question 2: Can (and how) does a historical perspective of China's electrical sector give indication of what might occur in the future in terms of GHG emissions?

\footnotetext{
A historical perspective (in this case gained from decomposition analysis) can provide insight on where policies have the most influence, and the lessons from this can in turn be used to show the possible impacts of future policies. Such a historical perspective can not, however, give an indication of how China's economy might progress into the future.
} 
For China's electrical sector, this historical perspective was studied in terms of both electricity demand and supply.

Decomposition analysis in the demand side of the electrical sector

Decomposition analysis of China's electrical sector illustrated key relationships between industrial growth and structure, energy efficiency, fuel shares and electricity demand.

During the five years analyzed (1998 to 2002), the two most important factors

contributing to changes in electricity demand were increases in industrial output and increases in energy efficiency. Structural changes towards manufacturing did not have a large impact on decreasing electricity demand. These conclusions suggest that efficiency gains should be carefully considered in turns of the business as usual scenario in China's electrical sector since a continuation of efficiency gains could be used to argue against the additionality of demand side CDM projects. However, recent evidence is that China's industrial sector has experienced a reversal of the energy intensity reductions seen historically. This suggests two things - methodologically there are risks in using past events to help indicate the future, and secondly, that there is an increased urgency to improve energy efficiency in China's economy if global manufacturing activity continues to shift towards the east.

Decomposition analysis in the supply side of the electrical sector

Decomposition analysis of China's electricity supply sector illustrated key relationships between generator efficiency, generation source mix, fuel shares, electricity losses realized during transmission, distribution and the generation of electricity, and the emissions intensity of electricity provided to end-users. Similar to demand, efficiency 
and not structural changes (i.e. changes in the fossil fuel or generation mix) led to the majority of changes in emission intensity. A number of economic and policy-level factors were then identified and studied to help explain these trends, showing that efforts of the central government to shut down smaller, less efficient plants was a major contributor to these efficiency gains, while the low cost and accessibility of coal in China has meant that coal remains the dominant fuel source in the sector. The results of the decomposition analysis in the supply sector also helped to inform the scenario formation process, specifically by studying how each factor could change under the scenario storylines, the emission impact of these changes, and the concurrent policy mix that might exist under each storyline in order for such changes to occur. This enabled a better understanding of the emission impact of economic and technological processes and policies that could affect China's electrical sector into the future.

Question 3: Can (and how) does the current circumstance of China's electrical sector give indication of what might occur in the future in terms of GHG emissions?

\section{Methods that rely on a current perspective of the electrical sector are not appropriate for measuring a future GHG baseline in terms of a country experiencing rapid economic growth and change such as in China.}

Since China's electrical sector is growing rapidly and new generation projects are very large, these processes combined can change the environmental profile of electricity provided to the end-user very rapidly. An example has been provided of how the Three Gorges dam project led to a sudden decrease in the emission intensity of electricity 
generated in south-central grids in China. If the build margin method were the baseline approach employed to generate the emission baseline for CDM projects in this region, fewer CDM projects would meet the environmental additionality criterion.

Question 4: Can (and how) does a simulation of China's electrical sector's future development give indication of what might occur in the future in terms of GHG emissions?

In order to simulate future emissions (in this case by using a dispatch model), careful consideration, study, and inclusion must be made of the factors and processes that will determine future emission levels. The inclusion of the regional and temporal dimensions of these factors advances the dispatch model's ability to accurately account for future GHG emissions.

In order to apply simulation analysis to understand the future development of China's electrical system, a framework was established to allow consideration of the economic, social, technological and political drivers affecting the sector into the future. These included regional differences in electricity demand, generation source mix, fuel availability, the transmission and distribution characteristics of the electrical system, and political or economical constraints affecting the electrical sector.

The building of this conceptual framework and the thorough review of historical factors that have occurred in China's electrical sector allowed three general storylines to be envisioned for the development of the sector to the year 2020. These scenarios 
included the 'business as usual' (BAU) scenario, and two alternative scenarios - an optimistic scenario and a conservative scenario. The BAU scenario was built upon established economic, energy, and environmental policies of China's central government and contained realistic assumptions about the future trajectory of China's economy and the world economic system. The optimistic scenario assumed faster economic growth, a stronger global economy, heightened trade, and faster increases in technology, while the conservative scenario assumed reduced trends in these parameters. The creation of these storylines then allowed plausible scenarios to be generated using the decomposition and dispatch approaches.

The scenario analysis showed that under the BAU the emission intensity of electricity will likely continue to fall in China while significant regional differences in the environmental quality of electricity will continue due to regional differences in technological levels, regional resource endowments and the subsequent generation source mix. Declining emission intensities will decrease the return from CDM projects over time in terms of the quantity (and hence dollar value) of emission reductions that might be expected. In addition, it can be expected that as the electrical sector grows, it will contribute to a greater share of China's burgeoning domestic demands for primary fuels, most notably coal and natural gas, while also contributing to an increasing share of China's total GHG emission inventory.

If a thorough review of the drivers that determine emissions from electricity generation were completed and conservative assumptions were made of the future level of these drivers, the dispatch modelling method could account for both regional and 
temporal dimensions of the factors considered and thereby provide an assessment of emission levels that could be considered regionally detailed and accurate.

Question 5: What lessons can be learned from the operationalization of the selected baseline methods?

Although arduous to use, the dispatch model is best able to ensure the overarching objective of environmental additionality since it is able to include regional and temporal dimensions in its analytical framework. The other two methods reviewed lack in their ability to consider important aspects of these dimensions. Nonetheless, a historical account as achieved through decomposition can provide very beneficial information to help ascertain the potential impacts of future policy choices or changes in economic processes.

The criterion of a baseline ensuring environmental additionality is central to the Kyoto Protocol's objective of reducing global GHG emissions from the BAU. Specific factors that contribute to this environmental additionality criterion have been considered: the ability of a baseline to include regional variation; the ability of a baseline to consider national circumstance; and, the rigour and accuracy of the baseline method. Reviewing each baseline method against these criteria helped to illustrate that the ability of the operating and build margin and the decomposition baseline techniques to meet the fundamental objective of additionality assessment is limited. For the operating and build margins, this limitation lies in its inability to consider temporal dynamics, while the main reason limiting decomposition analysis is its inability to consider regional details 
(particularly due to a lack of information for China). Subsequently, it was concluded that dispatch analysis is the most accurate method to help establish environmental additionality based on its ability to include the circumstances underlying the regional and temporal dynamics that affect an emissions baseline into the future.

There were also criteria related to the usability of the baseline method, particularly in terms of the data and tools available and required for the baseline analysis. Here, it was concluded that the data for operating and build margin analysis is relatively easy to obtain with the calculation of the baseline method from this data relatively straightforward. Alternatively, decomposition analysis requires a historical time series of data, with this often only available at the national scale. Considering dispatch analysis, this method proved to be both very data and knowledge intensive. Large amounts of data on the current configuration of China's electrical sector were required to populate the model, while both data and expert knowledge were needed to determine the future trajectory of drivers inputted into the model. This is one of the major limitations of the dispatch method - if a dispatch is not available for the electricity grid under study, or alternatively, one is used but its data has not been verified, this will mean either that the baseline won't be calculated or that it will be calculated wrong.

There are a number of key lessons in terms of ongoing development of baseline methodology. Decomposition provides a powerful tool to help understand quantitatively the potential emission impacts of policy and economic processes in the electrical sector, and could provide a suitable tool to help qualify the trajectories of emission baselines. Using a current perspective as gained from the operating and build margin baseline methodologies entails large risks in terms of inaccuracy in rapidly changing economies 
such as in China, and it is advisable that emission baselines not be constructed from these methods if considering a time frame of five years or longer. Finally, there should be more details asked for in the planning design documents that the UNFCCC requires for each CDM project in terms of the assumptions used to derive the baseline. Especially for baselines derived from the use of dispatch models, these assumptions can have very significant impacts on the emission intensity of electricity provided to the end-user.

\subsubsection{Key conclusions}

Overall, the assessments conducted in conjunction with the five research questions resulted in three key conclusions.

First, assessing the future of China's electrical sector is a very challenging task given the complexity of the system, its dynamics, and that it is heavily influenced by internal and external forces. Second, while there are several assessment approaches/methods available for measuring a future baseline, it has been demonstrated that the CDM assessment method chosen matters since the accuracy in the determination of additionality is influenced by the assessment method used. Of the baseline methods reviewed, it was found that dispatch modelling is best able to meet the environmental additionality criterion for the CDM, while decomposition analysis can contribute to a better understanding of the emission impacts of policies and processes operating within the BAU. Finally, it became evident that there are many choices to be made in regards to the sustainable development of China's electrical sector. The most promising options appear to be tied to increasing electrical end-use and generation technology and efficiency. In regards to the generation of electricity, the most promising and important 
technologies are related to clean coal technology since coal will most likely remain the dominant primary fuel for the foreseeable future.

\subsection{Expanding the research context}

The research completed was set in a specific context which effectively defined the scope of the investigation. This section reviews key dimensions of the research's scope and then suggests opportunities to expand the research's context in order to both improve the thoroughness of this assessment of China's electrical sector and CDM baseline methods. These are grouped into those concerning improving CDM baseline methodologies and those expanding the context of China's economy within CDM assessments and reflections on what this means for larger-scale issues as related to international environmental accords.

\section{Improving CDM baseline methodologies}

Opportunity \# 1: Establish a modelling framework for simulation analysis rather than using one owned by a separate organization.

The dispatch model employed in this research was developed, owned and operated by IC International, with its application directed by the researcher. This provided many opportunities (i.e. access to more advanced models, times savings, etc.) but this also meant that there was a limit in the number of variables that could be manipulated and outputs that could be generated from the model. 
Building a model rather than relying on one created by another party would enable a greater choice of both inputs and outputs. A greater choice of inputs would allow exploration of different possible futures for China's electrical sector, while a greater choice of model outputs could aid in the analysis of the scenarios generated. It would have been beneficial, for example, to have outputs on emissions and electricity generated organized into peak-load versus base-load and outputs on the configuration of generation technology to meet these different loads. However, this would have required significantly more computing time than was affordable for this project, as well as time to extract the information from the model and to compile this.

Opportunity \# 2: Expand the number of scenarios explored to match the IPCC (2000).

The three scenarios that were developed in the context of China's expected development path reinforced the need to consider a range of possible futures. These three scenarios effectively bounded the problem but more nuanced assessments (i.e. subsets of each of the three scenarios) went beyond the studies scope. Including more drivers and/or a different range of drivers would allow more scenarios to be envisioned and generated. In particular, it could prove beneficial to expand the number of scenarios generated to match the scenario families established by the IPCC (2000).

Opportunity \# 3: Strengthen working relationships with Chinese and/or Canadian partners. 
Transparent partnerships are a key component for the operation and evaluation of the CDM since this is a complex task. This research relied heavily on researcher-toresearcher collaboration but it would be useful to establish more formal institution-toinstitution partnerships, such as with the Energy Research Institute in China or university-based research units focused on energy and environment issues in China. A further opportunity thereby lies in strengthening, and in some cases establishing working relationships with both Chinese and Canadian partners in terms of some of the study components. Particularly, including Parties that could provide real, rather than hypothetical, CDM project case studies or to find Canadian derived technology examples that could be used in terms of project case study examples could prove of interest to both industry and to the ongoing development of the CDM.

\section{Expanding the context of China's economy within CDM assessments}

Opportunity \# 1: Expand the number of years included in historical analysis.

Electricity consumption and industrial sectoral data were analyzed only for the period spanning from 1998 to 2002, a period when China's economy was rapidly growing and energy intensity was decreasing. As is the case with any limited time-series, it can be very misleading to extrapolate backwards or forwards especially when drivers of change are volatile. In China's case, from the mid-1970's there are at least 3 notable shifts that occurred (e.g. from 1980 to approximately 1985, energy efficiency increased rapidly, from 1985 to 1990 emphasis was on increasing energy supply, while in the 1990's 
attention again was refocused upon energy efficiency). Unfortunately, the absence of reliable and comparable data for these periods has left these un-assessed and hence comparable and rigorous assessments over the past 30 years are not feasible. In addition, recent documentation of China's economic growth since 2002 suggests energy consumption intensities may be increasing (Shixian et al., 2005) but reliable data is not yet available to conduct an assessment which would compare 1998 to 2002 and the past the few years.

Opportunity \# 2: Strengthen linkages and synergies between international environmental agreements and domestic to international business investment and trade regimes.

This research has been set within the context of CDM assessment as defined the UNFCCC and Kyoto Protocol and hence focused on environmental additionality generally and more specifically the atmosphere. With China's rapidly changing economy coupled with new domestic and foreign strategies and regulations, the business as usual scenario becomes very difficult to project. For example, China's Renewable Energy Law (which was implemented in January 2006) requires $10 \%$ of the national energy supply to be from renewable sources by 2020 . The regional and local mechanisms that will facilitate this transformation are not yet clear and hence leave considerable uncertainty. The challenge that remains is how to best capture this uncertainty within the Chinese context, especially how it relates to specifying BAU scenario trajectories. Effectively, this will require moving the KP and UNFCCC out of its current atmosphere and climate 
focus and to develop more effective bridges between international environmental agreements and international business investment and trading regimes. 


\section{References}

Alcamo, J. and E. Kreileman (1996a). "Emission scenarios and global climate protection." Global Environmental Change 6(4): 305-334.

Alcamo, J., G. Kreileman, J. Bollen, G. van den Born, R. Gerlagh, M. Krol, A. Toet and H. de Vries (1996b). "Baseline scenarios of global environmental change." Global Environmental Change 6(4): 261-303.

Alcamo, J., P. Mayerhofer, R. Guardans, T. van Harmelen, J. van Minnen, J. Onigkeit, M. Posch and B. de Vries (2002). "An integrated assessment of regional air pollution and climate change in Europe: findings of the AIR-CLIM Project." Environmental Science \& Policy 5(4): 257-272.

Ang, B. W. (2004). "Decomposition analysis for policymaking in energy: which is the preferred method?" Energy Policy 32(9): 1131-1139.

Ang, B. W., C. Zhang and K.-H. Choi (1997). "Factoring changes in energy and environmental indicators through decomposition." Energy 23(6): 489-495.

Ang, B. W. and F. Q. Zhang (1999). "Inter-regional comparisons of energy-related CO2 emissions using the decomposition technique." Energy 24(4): 297-305.

Ang, B. W., F. Q. Zhang and K.-H. Choi (1998). "Factorizing changes in energy and environmental indicators through decomposition." Energy 23(6): 489-495.

Beech, H., B. Hua and S. Jakes (2006). Inside the pitchfork rebellion. Time. 167: 28-30.

Bernard, A., S. Paltsev, J. M. Reilly, M. Vielle and L. Viguier (2003). Russia's Role in the Kyoto Protocol. Boston, MIT Joint Program on the Science and Policy of Global Change: 1-24.

Bosi, M., A. Laurence, P. Maldonado, R. Schaeffer, A. Felipe Simões, H. Winkler and J. Lukamba (2002). Road-Testing Baselines for Greenhouse Gas Mitigation Projects in the Electric Power Sector. Paris, France, Organization for Economic Development and Cooperation, International Energy Agency.

British Petroleum (2004). British Petroleum Statistical Review of World Energy. London, British Petroleum. 2005: 1-44.

Canada (2006). Canada's Third National Report on Climate Change. Ottawa, Natural Resources Canada: 1-272.

CCBMC (2001a). Investment Opportunities in Coal Mine Methane Projects in Huainan Mining Area. Beijing, China Coalbed Methane Clearing House: 1-20.

CCBMC (2001b). Investment Opportunities in Coal Mine Methane Projects in Jiaozuo Mining Area. Beijing, China Coalbed Methane Clearing House.: 1-20.

CCBMC (2001c). Investment Opportunities in Coal Mine Methane Projects in Panjiang Mining Area. Beijing, China Coalbed Methane Clearinghouse: 1-20.

CCBMC (2001d). Investment Opportunities in Coal Mine Methane Projects in Yangquan Mining Area. Beijing, China Coalbed Methane Clearing House: 1-20.

CCMG (2004). Comments on Draft consolidated tools for demonstration of additionality. Republic of Colombia, Climate Change Mitigation Group: Ministry of Environment, Housing and Territorial Development.

CEPB (2004). China Electric Power Yearbook 2003. Beijing, China Electric Power Bureau: 1-712.

Chang, G. G. (2001). The coming collapse of China. New York, Random House. 
Chen, G., M. Firth and O. Rui (2006). "Have China's enterprise reforms led to improved efficiency and profitability?" Emerging Markets Review 7(1): 82-109.

Christiansen, A. C. (2003). "Convergence or divergence?: Status and prospects for US climate strategy." Climate Policy 3(4): 343-358.

CSP (1998-2004). China Statistical Yearbook. Beijing, China Statistical Press.

CSPIN (2006). China State Power Information Network - Power Sources, China State Power Information Network. 2006.

Demeritt, D. (2001). "The construction of global warming and the politics of science." Annals of the Association of American Geographers 91(2): 307-337.

den Elzen, M. G. J. and A. P. G. de Moor (2002). "Analyzing the Kyoto Protocol under the Marrakesh Accords: economic efficiency and environmental effectiveness." Ecological Economics 43(2-3): 141-158.

Dreborg, K. H. (1996). "Essence of backcasting." Futures 28(9): 813-828.

Dunaway, S. (2006). Rebalancing Economic Growth in China. International Herald Tribune. Washington, International Herald Tribune. January 11.

EEA (2003). EU greenhouse gas emissions rise for second year running. Copenhagen, European Environmental Agency.

EEA (2004). Annual European Community greenhouse gas inventory 1990-2002 and inventory report 2004 - draft. Brussels, European Environmental Agency: 1-167.

EEA (2005). An Environmental Scenarios and Prospective Studies Glossary. Copenhagen, European Environmental Agency. 2005.

EIA (2005). Annual Energy Outlook. Washington, Energy Information Association: 1253.

Ellerman, D. and A. Decaux (2001). Analysis of Post-Kyoto CO2 Emissions Trading Using Marginal Abatement Curves. Boston, Massachusetts Institute of Technology: 1-33.

Elliott, D., M. Schwartz, G. Scott, S. Haymes, D. Heimiller and R. George (2002). Wind Energy Resource Map of South-Eastern China. Golden, Colorado, National Renewable Energy Laboratory: 1-163.

Ellis, J. and M. Bosi (1999). Options for project emission baselines. OECD and IEA Information Paper. Paris, International Energy Agency: 1-60.

EnergyStrategy (2003). Draft Document for the Meeting of the Government of the Russian Federation May 22, 2003, "The Energy Strategy of Russia for the Period up to 2020" May 2003, as translated from Proekt k zasedaniyu Pravitel'stva Rossiiskoi Federatsii 22 maya 2003, "Energeticheskaya strategiya Rossii na period do 2020 goda", Moskva, mai 2003 god. Moscow: 1-117.

EPA (2004). EPA Modeling Applications Using The Integrated Planning Model. 2004.

Esparta, R. (2004). Comments on 'Draft Consolidated Tools for Demonstration of Additionality'. Sao Paulo, Ecoinvest: 1-2.

ESPON (2004). Spatial scenarios and orientations in relation to the ESDP amd Cohesion Policy. C. Vandermotten. Brussels, Belgium, European Spatial Planning Observation Network: 1-143.

EU-Commision (2006). Fourth national communication from the European community under the UN Framework Convention on Climate Change (UNFCCC). Brussels, Comission of the European Communities: 1-206. 
EU-Commission (2004). Questions \& Answers on Emissions Trading and National Allocation Plans. Brussels, European Commission: 1-7.

Fisher-Vanden, K., G. H. Jefferson, H. M. Liu and Q. Tao (2004). "What is driving China's decline in energy intensity?" Resource and Energy Economics 26(1): 77 97.

Garbaccio, R. F., M. S. Ho and D. W. Jorgenson (1999). "Why has the energy-output ratio fallen in China?" Energy Journal 20(3): 63-91.

Gnansounou, E. and J. Dong (2004). "Opportunity for inter-regional integration of electricity markets: the case of Shandong and Shanghai in East China." Energy Policy 32(15): 1737-1751.

Grubb, M. (2001). "Who's afraid of atmospheric stabilisation? Making the link between energy resources and climate change." Energy Policy 29(11): 837-845.

Grubb, M., J. Stern, B. Müller and Y. Safonov (2004). Russian energy and CO2 emission prospects: evidence from domestic analyses and international comparisons. London, The Royal Institute of International Affairs: 1-7.

Grübler, A. and N. Nakicenovic (2001). "Identifying dangers in an uncertain climate." Nature 15: 412.

Hamilton, C. and H. Turton (2002). "Determinants of emissions growth in OECD countries." Energy Policy 30(1): 63-71.

Hansen, J. (2005). "A slippery slope: How much global warming constitutes "dangerous anthropogenic interference"?: An Editorial Essay." Climatic Change 68(3): pp. $269-279$.

Hao, J. M., S. X. Wang, B. J. Liu and K. B. He (2001). "Designation of sulfur dioxide and Acid Rain Pollution Control Zones and its impacts on energy industry in China." Journal of Chemical Engineering of Japan 34(9): 1108-1113.

Harthan, R. (2004). Comments on consolidation of tools for demonstration of additionality. Germany, Energy and Climate Division: OkoInstitut: 1-2.

Hunhammar, S. (2001). "Transition to a sustainable society. A backcasting approach to modelling energy and ecology, Mulder, H.A.J., and Biesiot, W., Edward Elgar, Cheltenham, 1998, ISBN 1-85898-731-8, p. 287." Ecological Economics 38(3): 459.

ICF (2004a). China's Integrated Planning Model: Assumptions and Constructs. Washington D.C., ICF Consulting: 1-62.

ICF (2004b). Introduction to the Integrated Planning Model. Washington D.C., ICF Consulting: 1-28.

ICF (2005). China Power Market Database. Washington, ICF Consulting Incorporated: 123.

IEA (2002a). Developing China's Natural Gas Market. Paris, International Energy Agency: 1-372.

IEA (2002b). Russia Energy Survey 2002. Paris, International Energy Agency: 278.

IEA (2005). World Energy Outlook. Paris, International Energy Agency: 1-250.

IISI (2006). Steel Statistics, International Iron and Steel Institute. 2006.

IPCC (1996). Revised 1996 IPCC Guidelines for National Greenhouse Gas Inventories.

L. M. F. JT Houghton, B Lim, K Treanton, I Mamaty, Y Bonduki, and D. G. a. B.

C. (Eds), Intergovernmental Panel on Climate Change: 1-266. 
IPCC (2000a). Special Report on Emissions Scenarios. Cambridge, Cambridge University Press.

IPCC (2000b). Third Assessment Report of Working Group I: The Science of Climate Change. Cambridge, Cambridge University Press.

IPCC (2001). Climate Change 2001: Mitigation. New York, Cambridge University Press.

IPCC (2004). 16 years of scientific assessment in support of the climate change convention. Bonn, Intergovernmental Panel on Climate Change: 1-19.

Jefferson, G. H. and J. Su (2006). "Privatization and restructuring in China: Evidence from shareholding ownership, 1995-2001." Journal of Comparative Economics 34(1): 146-166.

Jie, Z. (2004). "West to East" Electric Power Transmitting" Strategy in China. Beijing, China Power Engineering Consulting Corporation.

Johnson, D. G. (2001). "The WTO and agriculture in China." China Economic Review 11(4): 402-404.

Jotzo, F. and A. Michaelowa (2002). "Estimating the CDM market under the Marrakech Accords." Climate Policy 2(2-3): 179-196.

Kartha, S., M. Lazarus and M. Bosi (2002). Practical Baseline Recommendations for Greenhouse Gas Mitigation Projects in the Electric Power Sector. Paris, International Energy Institute: $1-61$.

Kartha, S., M. Lazarus and M. Bosi (2004). "Baseline recommendations for greenhouse gas mitigation projects in the electric power sector." Energy Policy 32(4): 545566.

Kibona, E. (2004). Comments: Call for Inputs on Additionality Tools. Tanzania, Environmental Protection and Management Services (EPMS): 1.

Kohn, M. (1996). "Energy, environment and climate: Economic instruments." Energy \& Environment 7(2): 147-168.

Kroeze, C., J. Vlasblom, J. Gupta, C. Boudri and K. Blok (2004). "The power sector in China and India: greenhouse gas emissions reduction potential and scenarios for 1990-2020." Energy Policy 32(1): 55-76.

Law, G. (2006). Forward LNG prices in China. P. Steenhof. Houston, Wood Mackenzie.

Lazarus, M. (2003). The CDM Quantification Challenge: Time for a More Standardized Approach. Boston, Tellus Institute: 1-10.

Lazarus, M., S. Kartha and S. Bernow (2001). Project baselines and boundaries for project based GHG emission reduction trading, Tellus.

LBRL (2004). China Energy Database. Berkeley, Lawrence Berkeley Research Laboratory: 1-100.

Lee, C. F. and S. J. Lin (2001). "Structural decomposition of CO2 emissions from Taiwan's petrochemical industries." Energy Policy 29(3): 237-244.

Lovins, H. and A. Lovins (2001). "Natural Capitalism: Path to Sustainability?" Corporate Environmental Strategy 8(2): 99-108.

Manne, A. and R. Richels (2004). "US rejection of the Kyoto Protocol: the impact on compliance costs and CO2 emissions." Energy Policy 32(4): 447-454.

McKane, A., Z. Guijin, R. Williams, S. Nadel and V. Tutterow (2005). The China Motor Systems Energy Conservation Program: Establishing the Foundation for Systems Energy Efficiency. Berkely, Lawrence Berkeley National Laboratory: 1-6. 
METI (2004). Sustainable Future Framework on Climate Change: Draft of the Interim Report. Tokyo, Ministry of Economy, Trade and Industry of the Japenese Federal Government: 1-35.

Moe, A. and K. Tangen (2000). Russian quota sales: Not just a lot of hot air. London, Royal Institute of International Affairs: 110.

Munn, R. E. and A. R. Maarouf (1997). "Atmospheric issues in Canada." Science of The Total Environment 203(1): 1-11.

Nag, B. and J. K. Parikh (2005). "Carbon emission coefficient of power consumption in India: baseline determination from the demand side." Energy Policy 33(6): 777786.

Nakicenovic, N. (2000). "Greenhouse Gas Emissions Scenarios." Technological Forecasting and Social Change 65(2): 149-166.

Nazarov, I. M., Y. U. Izrael, A. F. Yakovlev, Y. U. Anokhin, L. I. Boltneva, M. I. Gytarsky, G. V. Gruza, V. I. Egorov, R. T. Karaban, A. I. Nakhutin and E. M. Nakhutin (2002). Third National Communication of the Russian Federation to the United Nations Framework Convention on Climate Change. Moscow.

Ogilvy, J. A. (2000). China's futures: scenarios for the world's fastest growing economy, ecology, and society. San Francisco, Jossey-Bass.

Paik, K. H. (2004). The implications of China's gas expansion towards the natural gas market in Asia. London, Chatham House: 1-130.

Parikh, J. K. (1998). "The emperor needs new clothes: long-range energy-use scenarios by IIASA-WEC and IPCC." Energy 23(1): 69-70.

Park (1992). "Decomposition of industrial energy consumption: An alternative method." Energy Economics 14: 265:270.

Paul, S. and R. N. Bhattacharya (2004). "CO2 emission from energy use in India: a decomposition analysis." Energy Policy 32(5): 585-593.

Powell, R. L. (2002). "CFC phase-out: have we met the challenge?" Journal of Fluorine Chemistry 114(2): 237-250.

PRC (2006). The Renewable Energy Law. Beijing, People's Republic of China: 1-11.

Prestowitz, C. (2005). Three Billion New Capitalists. New York, Perseus Books Group.

Qingyi, W. (2004). Coal Industry in China: Evolvement and Prospects. San Francisco, Nautilus Institute.

Ringius, L. (2004). Analysis of the Additionality Tool (with project examples). CDM Methodologies and Project Design: Workshop for CDM Project Developers, Buenos Aires.

Robinson, J. (2003). "Future subjunctive: backcasting as social learning." Futures 35(8): 839-856.

Saran, A. and C. Guo (2005). "Competing in the global marketplace: The case of India and China." Business Horizons 48(2): 135-142.

Sathaye, J., S. Murtishaw, L. Price, M. Lefranc, J. Roy, H. Winkler and R. SpaldingFecher (2004). "Multiproject baselines for evaluation of electric power projects." Energy Policy 32(11): 1303-1317.

Seng-Kee, W. (2006). Managing the Supply of LNG in Asia, from a Major's Perspective. 11th Asia Natural Gas Markets Conference, Singapore.

SEPA (1995). The National Ninth Five-Year Plan for Environmental Protection. Beijing, State Environmental Protection Administration. 
SEPA (2002). The National Tenth Five-Year Plan for Environmental Protection. Beijing, State Environmental Protection Administration.

Seyfang, G. (2003). "Environmental mega-conferences--from Stockholm to Johannesburg and beyond." Global Environmental Change 13(3): 223-228.

Shixian, G., G. Zhicheng, Y. Qing and G. Yuan (2005). China's National Comprehensive Energy Strategy and Policy. Beijing, Development Research Center: 1-52.

Shrestha, R. M. and G. R. Timilsina (1997). "SO2 emission intensities of the power sector in Asia: Effects of generation-mix and fuel-intensity changes." Energy Economics 19(3): 355-362.

Shrestha, R. M. and G. R. Timilsina (1998). "A divisia decomposition analysis of NOx emission intensities for the power sector in Thailand and South Korea." Energy 23(6): 433-438.

Sinton, J. and D. Fridley (2003). "A guide to China's energy statistics : Sinton, J. E. and Fridley, D. G. The Journal of Energy Literature, 2002, VIII, (1), 20-35." Fuel and Energy Abstracts 44(2): 117.

Sinton, J. and L. Jiang (2005). Energy Issues in China and the Policy Challenges Ahead. Beijing, Lawrence Berkeley Research Laboratory: 1-125.

Sinton, J. and M. Levine (1994). "Changing energy intensity in Chinese industry." Energy Policy 22(3): 239-255.

Sinton, J. E., M. D. Levine and W. Qingyi (1998). "Energy efficiency in China: accomplishments and challenges." Energy Policy 26(11): 813-829.

Steenhof, P. (2006a). "Decomposition for emission baseline setting in China's electricity sector." Energy Policy 34(8).

Steenhof, P. (2006b). "Decomposition of electricity demand in China's industrial sector." Energy Economics 28.

UNFCCC (2001). The Marrakech Accords and the Marrakech Declaration. Marrakech, United Nations Framework Convention on Climate Change: 1-275.

UNFCCC (2005). Approved Methodologies. Bonn, Gernanyt, United Nations Framework Convention on Climate Change Executive Board. 2005: 1.

UNFCCC (2006a). Annex I National Communications, UNFCCC. 2006.

UNFCCC (2006b). Greenhouse Gas Inventory Data, UNFCCC. 2006.

Viguier, L. L. (2004). "A proposal to increase developing country participation in international climate policy." Environmental Science \& Policy 7(3): 195-204.

Wang, B. (2003). Electricity Reform in China: Past, Present and Future. Energy and Environment Seminar, Cambridge, UK.

WB (2004). Clean Development Mechanism in China: Taking a proactive and sustainable approach. Washington, D.C., The International Bank for Reconstruction and Development/The World Bank: 1-199.

Wilk, R. (2002). "Consumption, human needs, and global environmental change." Global Environmental Change 12(1): 5-13.

WNA (2005). Nuclear Power in China. London, U.K., World Nuclear Association. 2006.

Wu, L., S. Kaneko and S. Matsuoka (2005). "Driving forces behind the stagnancy of China's energy-related CO2 emissions from 1996 to 1999: the relative importance of structural change, intensity change and scale change." Energy Policy 33(3): 319-335. 
Yamaguchi, K. and K. Cho (2004). Natural Gas in China. Tokyo, Institute of Energy Economics: 1-10.

Youguo, H. (2004). China 's Coal Demand Outlook for 2020 and Analysis of Coal Supply Capacity. Beijing, China Coal Industry Development Research and Consulting Co. Ltd. (China Coal Information Institute).

Zhang, C. (2002). "Impact on global warming of development and structural changes in the electricity sector of Guangdong Province, China." Fuel and Energy Abstracts 43(1): 50 .

Zhang, C. (2003). "Why did the energy intensity fall in China's industrial sector in the 1990s? The relative importance of structural change and intensity change." Energy Economics 25(6): 625-638.

Zhang, C., T. Heller and M. May (2005). "Carbon intensity of electricity generation and CDM baseline: case studies of three Chinese provinces." Energy Policy 33(4): 451-465.

Zhang, K. H. and S. F. Song (2003). "Rural-urban migration and urbanization in China: Evidence from time-series and cross-section analyses." China Economic Review 14(4): 386-400.

Zhufeng, Y. and Y. Jie (2001). Policy Study in the Development of Clean Coal Technology. Cleaner Production in China, Beijing, China. 


\section{APPENDIX A}

1 COAL MINE METHANE ELECTRICITY GENERATION (CMM),..............................................219

1.1 GENERAL INFORMATION AND NATIONAL CIRCUMSTANCE ..............................................................219

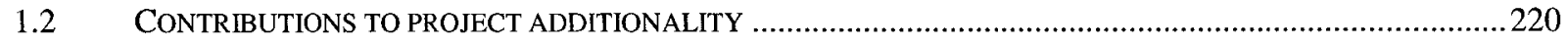

2 SUPER-CRITICAL COAL-FIRED POWER PROJECT (CCT) ..........................................................221

2.1 GENERAL INFORMATION AND NATIONAL CIRCUMSTANCE ................................................................22

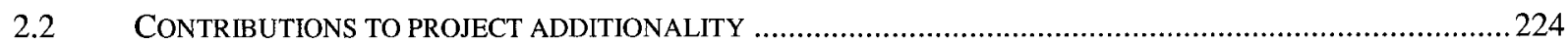

3 INSTALLATION OF COMBINED CYCLE (TRI GENERATION) NATURAL GAS

GENERATING PLANT PEAKING PLANT (G3-CCT) ..............................................................................224

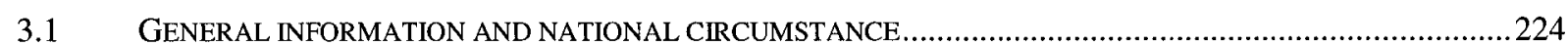

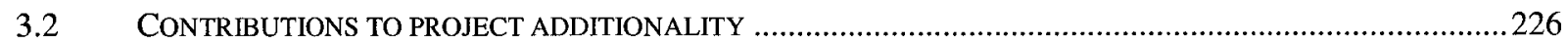

4 INSTALLATION OF LARGE WIND FARM (WG) .................................................................................227

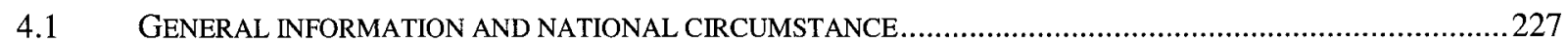

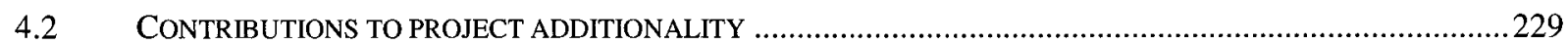

5 INSTALLATION OF SMALL HYDRO RUN OF THE RIVER HYDRO-ELECTRIC

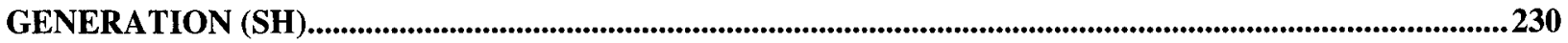

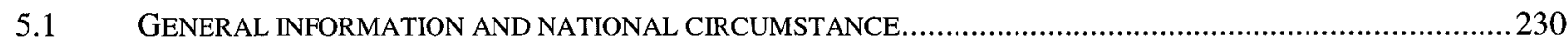

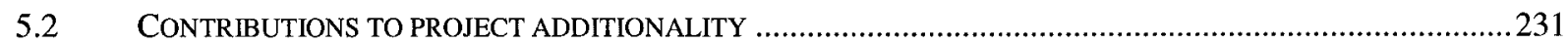




\section{Coal mine methane electricity generation (CMM)}

\subsection{General information and national circumstance}

In coal production, a large amount of methane $\left(\mathrm{CH}_{4}\right)$ is released to the atmosphere from both origins in coal seams and the coal structure. This coal mine methane (referred to as CMM throughout) represents a potential significant source for CDM projects in China.

First, as China produces the most coal in the world (and likely to increase with China's economic growth), reducing its CMM emissions now and into the future is a key part of reducing the country's GHG emissions. In 2000, global CMM emissions totaled nearly $440 \mathrm{MMtCO}_{2} \mathrm{e}$, or about 30.8 billion cubic meters (BCM) of methane (EPA, 2003). China contributed to nearly 40 percent of these emissions, or about $12 \mathrm{BCM}$ of methane. The second reason why CMM offers a large potential source for CDM activity is the economic benefits of the methane. CMM based CDM projects align closely with many of China's sustainable development initiatives and could help spur much needed technological investment into China. Currently, the effectiveness of CMM drainage and utilization is limited due to a general lack of gas pipeline infrastructure needed to transport gas, outdated gas drilling and ventilation systems, a lack of preventative maintenance or equipment, use of inappropriate technology unsuited to geological and mining conditions, and inadequate monitoring and control systems. CDM project activity could help attract investment to any or all of these areas, increasing management capacity and attracting technology transfer. A fourth reason increasing the applicability of these projects, and one directly linked to China's sustainable development, is that the installation of internationally recognized ventilation and gas extraction techniques will 
significantly increase mine safety. Coal mine safety has historically been very low in China with gas explosions being a major cause of death and injury. In 2000, of the 5,798 mining deaths in China, 3,132 were from methane gas explosions in coal mines (LBRL, 2004).

In most cases, potential projects based upon CMM are easily obtainable and represent low cost emission reduction opportunities. Examples include projects for power production, co-firing of methane gas in boilers, district heating, mine heating, coal drying, and usage of natural gas as a fuel for vehicles. Of these types, electricity generation ranks the most profitable and feasible both environmentally and financially (WB, 2004). For one, technology has progressed to a level now that even low-grade methane extracted from coal mines can be condensed and used for thermal power generation. The generation of electricity from these resources is also highly attractive because the infrastructure and market for this is readily available; using generation technology and tapping into local grids, the methane can be used quickly to produce revenue, unlike other uses that may require development of end markets or costly transportation. Nonetheless, there are relatively few domestically initiated power generation schemes in China due to the high capital cost of the plant and the historic low price of electricity.

\subsection{Contributions to project additionality}

Shultz (2005) highlights a number of general attributes that increase the additionality of potential CMM based CDM projects in China: 
- Comprehensive utilization of CMM is rare in China, with only 0.5 BCM used in 2002;

- There is no requirement for coal mines to utilize CMM drained and there is no requirement expected in the foreseeable future for mines to capture and utilize CMM;

- There have been some relatively short-lived CMM power generation schemes at coal mines that have failed due to lack of finance, technical support and maintenance. The CER revenue and technology transfer associated with the CDM may alleviate this;

- The economic attractive option, without the incentives associated with CER revenue, is to vent $\mathrm{CMM}$ into the atmosphere, and use funds for purposes such as increasing coal production capacity;

- Financing CMM schemes is considered higher risk due to past negative experiences in for failed CMM projects and the more profitable investments available for coal production.

- Currently there are few electricity generation schemes in China associated with CMM, There have been a number of related CDM projects, but the technology behind these is definitely not prevalent in China.

\section{Super-Critical Coal-Fired Power Project (CCT)}

\subsection{General information and national circumstance}

Clean coal technology has tremendous potential in China. Currently over 95 percent of all fossil fuel fired generators in China are powered by coal, with the majority of these 
located in the regions with high concentrations of population. Even though it has this great potential, only a minor amount of total coal-fired generation is CCT, and of this, nearly all in imported. Although domestic capacity is being developed, China will still depend on foreign financing for these for at least the upcoming decade because domestic resources will take a long time to advance to a high enough level to meet the country's demands for the technology, and due to the higher financing requirements for these.

This project case study is based upon a super-critical coal-fired power project (referred to as SC-CF hereafter) with total capacity of $3,600 \mathrm{MW}$ providing baseload capacity. This project type was initially identified by the World Bank (2004) as one of the most eligible CDM projects available to the China market when considering its potential scale of application and its environmental and economical characteristics. SCCF can be classified as clean coal technology as it is more fuel-efficient, burning coal at higher temperatures and higher pressure and with less oil input than existing plants and therefore results in significantly reduced emissions of both GHG and other air pollutants (particularly $\mathrm{SO}_{2}$ ).

The particular project case study included is a six-unit generation station, both $600 \mathrm{MW}$ in size, fueled by higher-grade bituminous coal. Table 1 provides a comparison between SC-CF to its less efficient, more polluting counterpart, the sub-critical coal fired plant for the same size of plant, in terms of efficiency and standard rates of pollution emission measured by electricity generated, as well as some pertinent financial parameters. 
Table 23. Comparing sub and super critical coal electricity generation

\begin{tabular}{|l|r|r|}
\hline \multicolumn{1}{|c|}{ Parameter } & \multicolumn{1}{|c|}{$\begin{array}{c}\text { Super-critical } \\
\text { coal-fired } \\
\text { power project }\end{array}$} & $\begin{array}{c}\text { Sub-critical } \\
\text { coal-fired } \\
\text { power project }\end{array}$ \\
\hline Capacity $(600 \mathrm{MW})^{*} 6$ units & 3,600 & 3,600 \\
\hline Capacity factor $(\%)$ & $280-00 \%$ & $80.00 \%$ \\
\hline $\begin{array}{l}\text { Fuel consumption (grams } \\
\text { c/KWh) }\end{array}$ & & $340-420$ \\
\hline Thermal efficiency (\%) & 40 & 32 \\
\hline $\begin{array}{l}\text { Carbon emission rates per unit } \\
\text { generated (kg CO } 2 \text { e/KWh) }\end{array}$ & $5.78-0.89$ & $0.94-1.16$ \\
\hline $\begin{array}{l}\text { Sulphur emission rates per unit } \\
\text { generated (g SO } / \text { KWh) } \\
\text { (no_abatement) }\end{array}$ & $5.51-6.30$ & $6.69-8.26$ \\
\hline Annual $\mathrm{CO}_{2} \mathrm{e}$ emissions (mtonnes) & $19.50-22.37$ & $23.78-29.35$ \\
\hline Annual $\mathrm{SO}_{2}$ emissions (ktonnes) & $139-159$ & $169-208$ \\
\hline Capital costs (USD/KW) & 1,200 & 1,050 \\
\hline O\&M Costs (USD/KWh) & 0.29 & 0.25 \\
\hline Project life time & 20 & 20 \\
\hline
\end{tabular}

\footnotetext{
${ }^{1}$ Sub critical unit fuel consumption rates are based upon the range for the different provinces as given in China's Electricity Power Yearbook (please see page 356 for details). This assumes therefore that the fuel consumption rates in the Yearbook are mainly reflective of sub critical units.
} 
As can be seen, this technology type results in a reduction of nearly 22 percent for $\mathrm{CO}_{2} \mathrm{e}$ when compared to the characteristics of the average sub-critical generation unit.

\subsection{Contributions to project additionality}

Project developers in China have experienced financial and technological hurdles in the last number of years as financing constraints exist for the commercialization for CCT (Zhufeng et al., 2001). Consequently, the additional revenue achieved by carbon sales associated with CERs increase the marginal cost feasibility for this type of capacity, contributing to their subsequent eligibility for CDM classification.

\section{Installation of combined cycle (tri generation) natural gas generating plant peaking plant (G3-CCT)}

\subsection{General information and national circumstance}

By 2000 , there was over $28 \mathrm{GW}$ of cogeneration in China, 12.6 percent of the total electricity generation potential in the country (LBRL, 2004). Cogeneration has experienced relatively fast growth rates over the last ten to twenty years because of domestic interest in energy conservation and the many environmental and energy laws encouraging the development of such technology. One of the most relevant energy laws was the "Energy Conservation Law of China", which emphasized the development of cogeneration for heat and power (UNESCAP, 2005). A related policy established in 1998 (found within the "Official Reply to Issues Concerning the Acid Rain Control Zone and the Sulfur Dioxide Pollution Control Zone by the State Council") specified that for cities 
found within the two control zones, cogeneration on electricity generators was a requirement.

This project case study (the combined cycle turbine natural gas generating peaking plant (referred to as G3-CCT interchangeably)), differs from commonly implemented cogeneration units as it uses thermal energy from natural gas combustion to first drive a turbine generator, waste heat to generate steam to drive a steam turbine, and lower grade waste heat to either heat water for space heating or in space cooling processes. Therefore, emission reductions would result from three areas rather than just one (or two in the case of cogeneration); emission reductions associated with newly generated electricity, emission reductions associated with using natural gas derived space cooling versus electrical space cooling, and emission reductions from using natural gas derived space warming through warm water circulation. For the first two of these, this would displace electricity from the regional electricity grid, while for the third it is assumed that this is coal-based space heating.

The technology of G3-CCT provides an energy efficient, economical and compatible way to use energy sources in terms of end use and the quality of the energy source used. This is because in this system, exhaust heat from the initial thermal energy cycle to produce electricity, normally treated as exhaust waste, is recovered, extracted, and converted into stream in order to drive a steam turbine for electricity generation, while lower grade heat is either used for space heating or space cooling in the summer. Such an approach thereby would be able to produce electricity through a combined gassteam combined cycle, while providing heat/cooling services. This would result in a 
combined integrated efficiency of between 70 and 80 percent, with an electricity generation efficiency of $35-40$ percent.

This technology is also particularly relevant due to the geographical layout of the typical Chinese city and the relevance of a technology that could jointly provide electricity and thermal loads for heat, industrial processes, etc. Many of China's largest cities are well-organized grids, with massive housing units organized in stack units, some approaching 20 to 30 stories high. Industry is located in large, often tightly organized industrial parks, such as is found in Beijing's northern reaches. Such characteristics mean that distribution of cooling and heating services associated with a CDM project would not necessitate large outlays of financial resources, thereby increasing the cost feasibility of these projects, and therefore increasing their attraction for potential investors.

As suggested, such a project will have positive implications on power shortages in China, and particularly for peak power. Over the past decade or so, peak electricity has been steadily increasing in China. However, throughout China's electricity grids large coal fired generators dominate with little peaking capacity available. As a result, in the last number of years China has began to experience brownouts during hours of peak demand. These grew in severity in the summers of 2003 and 2004, diminishing industrial production in many regions.

\subsection{Contributions to project additionality}

The additionality of such potential projects is increased by the price volatility and generally high prices for natural gas seen in China, as well as a general lack of domestic capability to produce this technology. Fuel costs mean that operational costs are higher 
than coal-fired plants, and as has been described, domestic production capabilities are largely focused upon coal generation. Therefore, CER sales could contribute significantly to the financial viability of these technologies. This latter point is strengthened by the three possible sources of CER revenue (i.e. from increased efficiency of power generation, using waste and cooling potential).

\section{Installation of large Wind Farm (WG)}

\subsection{General information and national circumstance}

China is well endowed with wind resources, with the exploitable amount of wind energy reserve estimated to be at $253 \mathrm{GW}$ (Lew, 2000). There is currently $567 \mathrm{MW}$ of wind generation capacity in China ( 0.22 percent of total potential exploitable capacity), ranging from smaller size wind turbines usually $100 \mathrm{~W}$ in size (there are 170,000 of these), to larger scale wind farms (LBRL, 2004). Geographically, China's wind resource is concentrated in the north and west regions and along the east coast. The wind resource is suited for both remote village power development and for large-scale, grid-connected electricity production. However, for the most part over the last 50 years the approximately 175,000 wind units in China are small, less than $1 \mathrm{KW}$ in size, and used in villages or by herdsmen to generate remote power (ref). The cumulative capacity of these equals $17 \mathrm{MW}$, less than one single large wind farm. For example, in 2000 the largest wind farm (in Xianjing, North China) had total installed capacity of 71MW (LBRL, 2004). By 2003, there were 1,043 of such wind farms, with an average size of $544 \mathrm{KW}$. 
Although the country was well below its planned wind capacity, China does have ambitious wind development plans for the near future. The Tenth Five-Year Plan (20012005) established a target to have 1,500 MW of wind capacity by in 2005 (PRC, 2001). Most of the current and future development is expected to occur in China's north and eastern regions, with little wind capacity developed in the south and central regions due to a lack of suitable wind resources in these areas.

Following the efforts in the country in the recent past to develop and build wind capacity, the PRC is emphasizing development of domestic capabilities to harness future development of its wind resources. Currently, most of the domestic capabilities in this regard lie in the ability to manufacture and implement small-scale wind generation units, with little capacity to develop larger scale units. However, into the future China will look to develop larger scale technology suitable for grid-connected systems, such as wind farms in the 10-30 MW size range.

A comparable CDM project is the installation of the Huitengxile Windfarm project currently being implemented into North China's Electricity Grid (ITPower, 2004). The main investor of this particular CDM project is the Dutch Government through their CERUPT program, while the project developer is the Inner Mongolia Windpower Corporation. The project involves the installation of 22 turbines providing $25.8 \mathrm{MW}$ of capacity, thereby generating $59.2 \mathrm{GWh}$ of electricity per year and resulting in an estimated emission reduction of 513,914 tCO2e in the period 2004-2012. The project will contribute to sustainable development in China by helping Chinese firms establish technical and management capacities to develop such large-scale wind farms. It will also 
lead to a reduction in local and regional air pollution since wind generated electricity results in zero emissions of these.

\subsection{Contributions to project additionality}

A significant factor inhibiting the development of wind resources in the past has been the higher capital costs associated with the generation type, much higher than of coal, hydro or gas. For such reasons, CERs generated from the CDM can improve the cost feasibility of these projects. Historically, a range of financing mechanisms has been used to help shoulder the higher incremental costs of wind generation (Liu et al., 2002). More recently, China has tried to increase its domestic capacity for both production and financing of wind projects. In 2000, the State Development Planning Commission issued policy that would force wind project developers to use at least 40 percent domestic content in the project components in an attempt to increase local manufacturing of these (ITPower, 2004). The CDM wind project invested in by the Dutch government, for example, was able to meet this condition by using 100 percent domestically produced wind generation components.

The general attributes that increase the additionality of potential large scale wind farm CDM projects in China therefore can be summarized as:

- China still lacks the domestic capacity to design, manufacture and implement large scale wind farms.

Nonetheless, it should be noted that the new Renewable Energy Law established on January 1,2006 , complicates the additionality of this technology as wind generation 
becomes a more prominent component of the business as usual. This, however, will only be able to be determined and argued when regional targets and plans are established for the REL and if domestic capabilities increase in terms of wind farms rather than small scale capacity.

\section{Installation of small hydro run of the river hydro- electric generation $(\mathrm{SH})$}

\subsection{General information and national circumstance}

From an environmental and energy perspective, the harnessing of water resources decreases air pollution and GHG emissions, while decreasing domestic dependence on foreign energy markets. China is endowed with some of the richest water resources in the world (17.6\% of the global total) of which exploitable medium and small hydropower resources equal 139GW (Anon, 2004). Most of these resources are located in the vast mountainous areas. Over the last twenty years, the PRC has placed high importance on the electrification of rural areas and therefore has spearheaded the development of many of these resources. By the mid 1990's the total installed capacity of small hydropower amounted to over 19GW with electric output to 64TWh per year (LBRL, 2004).

Hydropower supplies electricity to 300 million people in about 800 counties. Because of the geography of China, and the ensuing policy emphasis on development of small hydro capacity, China has accumulated substantial experience in developing, constructing and managing rural small hydro development. 


\subsection{Contributions to project additionality}

A number of factors contribute to the additionality of this technology. First, although small hydro has been widely implemented in the past, in many cases the implementation of this technology still relies on government funding. CER revenues would therefore contribute towards the financial viability of this technology. Additionally, clearly SH has many sustainable development attributes attached to it: it provides stable sources of power, reduces air pollution, creates local employment, and reduces GHG emissions. Nonetheless, similar to wind generation, it should be noted that the new Renewable Energy Law established on January 1, 2006, complicates the additionality of this technology as it become a more prominent component of the business as usual. This, however, will only be able to be determined and argued when regional targets and plans are established for the REL.

\section{References}

Anon (2004). "Small Hydropower in China." Refocus December.

EPA (2003). Assessment of the world wide market potential for oxidixing coal mine ventilation air methane. Washington, Environmental Protection Agency: 118.

ITPower (2004). Baseline Study for a Windfarm at Huitengxile, Inner Mongolia, China: Consultation Report. Huitengxile, Inner Mongolia Windpower Corporation: 1-32. LBRL (2004). China Energy Database. Berkeley, Lawrence Berkeley Research Laboratory: $1-100$. 
Lew, D. J. (2000). "Alternatives to coal and candles: wind power in China." Energy Policy 28(4): 271-286.

Liu, W.-Q., L. Gan and X.-L. Zhang (2002). "Cost-competitive incentives for wind energy development in China: institutional dynamics and policy changes." Energy Policy 30(9): 753-765.

PRC (2001). Tenth Five-Year Plan on National Economy and Social Development. Beijing, People's Republic of China.

Shultz, K. (2005). The impact of certified emission reductions on the Chinese coal mine methane industry. London, Climate Mitigation Works Ltd.: 1-9.

UNESCAP (2005). Energy Conservation Law of China (Presidential Act of the People's Republic of China No. 90)(1 November 1997). New York, United Nations Economic and Social Commission for Asia and the Pacific. 2005.

WB (2004). Clean Development Mechanism in China: Taking a proactive and sustainable approach. Washington, D.C., The International Bank for Reconstruction and Development/The World Bank: 1-199.

Zhufeng, Y. and Y. Jie (2001). Policy Study in the Development of Clean Coal Technology. Cleaner Production in China, Beijing, China. 


\section{APPENDIX B}

\section{CLEAN DEVELOPMENT MECHANISM \\ PROJECT DESIGN DOCUMENT FORM (CDM-PDD) \\ Version 02 - in effect as of: 1 July 2004)}

\section{CONTENTS}
A. General description of project activity
B. Application of a baseline methodology
C. Duration of the project activity / Crediting period
D. Application of a monitoring methodology and plan
E. Estimation of GHG emissions by sources
F. Environmental impacts
G. Stakeholders' comments

\section{Annexes}

Annex I: Contact information on participants in the project_activity 
SECTION A. General deseription of proiect activity.

A.1 Title of the project activity:

Wind Farm Jiangsu Province, East China

A.2. Description of the project activity:

This project is a grid connected wind farm located in Jiangsu Province, China. The project is $50 \mathrm{MW}$ in capacity, and operates at an annual capacity factor of $30 \%$. This means the wind farm will generate electricity approximately 2,600 hours per year, or $131 \mathrm{GWh}$ of electricity per annum. All project technology will be developed domestically within China.

\section{A.3. Project participants:}

Paul Steenhof, Carleton University (trying to raise departmental funds)

Changzhou Wujin Chemical \& L. Industries Corp. Ltd., Jiangsu Province, China (local manufacturer)

A.4. Technical description of the project activity: 
The technical details of the project are summarized in the following table:

\begin{tabular}{|l|r|}
\hline & \multicolumn{1}{|c|}{$\begin{array}{c}\text { Wind } \\
\text { farm }\end{array}$} \\
\hline Fuel & Wind \\
\hline Capacity (MW) & 50 \\
\hline $\begin{array}{l}\text { Capacity factor } \\
\text { (\%) }\end{array}$ & 0.3 \\
\hline Hours per year & 2,628 \\
\hline Efficiency (\%) & NA \\
\hline $\begin{array}{l}\text { Annual } \\
\text { generation (GWh) }\end{array}$ & 131 \\
\hline $\begin{array}{l}\text { Project CO2 } \\
\text { emissions } \\
\text { (tonnes) }\end{array}$ & 0 \\
\hline $\begin{array}{l}\text { Project emission } \\
\text { intensity } \\
\text { (kgCO2/KWh) }\end{array}$ & 0.000 \\
\hline
\end{tabular}

A.4.1. Location of the project activity:

Jiangsu Province, China
A.4.1.1.
Host Party(ies):

None yet
A.4.1.2.
Region/State/Province etc.:

The proposed project is located in Suzhou, Jiangsu.
A.4.1.3.
City/Town/Community etc: 
The proposed project is located in Suzhou, Jiangsu
A.4.1.4.
Detail of physical location, including information allowing the

unique identification of this project activity (maximum one page):

A.4.2. Category(ies) of project activity:

Wind

A.4.3. Technology to be employed by the project activity:

Wind turbine

A.4.4. Brief explanation of how the anthropogenic emissions of anthropogenic greenhouse gas (GHGs) by sources are to be reduced by the proposed CDM project activity, including why the emission reductions would not occur in the absence of the proposed project activity, taking into account national and/or sectoral policies and circumstances:

Currently, China has significant wind resource development for small scale individual wind turbines, primarily used in the agricultural sector. However, China lacks sufficient capacity to build and implement large scale wind farms such as proposed in this project. Therefore, the emission reductions associated with this project would be additional to those otherwise that would occur. These emission reductions will particularly occur 
since wind is a zero carbon form of energy while the electricity provided to end-users in the Jiangsu grid is relatively carbon intensive (approximately $0.83 \mathrm{~kg} \mathrm{CO} 2 / \mathrm{KWh}$ in 2010 based on the BAU). Therefore, wind generated electricity will result in a reduction of $\mathrm{CO} 2$ from the BAU.

A.4.4.1. Estimated amount of emission reductions over the chosen crediting period:

The estimated amount of emission reductions over the chosen credit period would equal 1.482 million metric tonnes of carbon dioxide

A.4.5. Public funding of the project activity:

0 dollars

SECTION B. Application of a baseline methodology

B.1. Title and reference of the approved baseline methodology applied to the project activity:

Multi project baseline methodology - dispatch analysis

B.1.1. Justification of the choice of the methodology and why it is applicable to the project activity: 
This baseline method meets the majority of criteria established by the CDM Executive Board for baseline emission methodology. Above all, it properly ensures the environmental additionality criterion for emission baselines as it accomplishes the following things: It accounts for national and regional circumstance; it accounts for geographical applicability, particularly in terms of regional generation characteristics and regional electricity transfers; and, conservative assumptions are used in deriving the BAU scenario compiled for this PDD. It is particular applicable to this study since it can isolate the emission intensity for the specific electricity grid for which this project will be implemented into from present to the year 2020 .

B.2. Description of how the methodology is applied in the context of the project activity:

This method is applied by taking the annual grid emission intensity (the baseline) from present to the year and subtracting this from the project emission factor. These annual values are cumulated to determine total emission reductions over the course of the project activity.

B.3. Description of how the anthropogenic emissions of GHG by sources are reduced below those that would have occurred in the absence of the registered CDM project activity: 
Anthropogenic emissions of GHG is reduced below those would have occurred since wind is a zero emission source for electricity, and the grid region where this project will be implemented is a high emission intensive grid.

B.4. Description of how the definition of the project boundary related to the baseline methodology selected is applied to the project activity:

The project boundary is the electricity provided to the grid. The emission reductions do not account for materials and energy used to develop the wind generation capacity. This relates to the baseline methodology since the boundary of this is the emissions of GHGs from all electricity provided to the grid.

B.5. Details of baseline information, including the date of completion of the baseline study and the name of person (s)/entity (ies) determining the baseline:

The date of completion of the baseline study was April 1, 2006. The person who determined the baseline was Paul Steenhof (psteenho@connect.carleton.ca)

SECTION C. Duration of the project activity / Crediting period

C.1 Duration of the project activity:

C.1.1. Starting date of the project activity: 
June 1, 2006 (expected)

C.1.2. Expected operational lifetime of the project activity:

December 31, 2020.

C.2 Choice of the crediting period and related information:

June 1, 2006 to December 31, 2020.

C.2.1. Renewable crediting period

C.2.1.1. Starting date of the first crediting period:

C.2.1.2. Length of the first crediting period:

C.2.2. Fixed crediting period:

C.2.2.1. Starting date:

June 1, 2006 
C.2.2.2. Length:

13 years, 6 months

SECTION D. Application of a monitoring methodology and plan

D.1. Name and reference of approved monitoring methodology applied to the project activity:

Multi-project baseline analysis - Jiangsu Province - Dispatch analysis

D.2. Justification of the choice of the methodology and why it is applicable to the project activity:

This baseline method meets the majority of criteria established by the CDM Executive Board for baseline emission methodology. Above all, it properly ensures the environmental additionality criterion for emission baselines as it accomplishes the following things: It accounts for national and regional circumstance; it accounts for geographical applicability, particularly in terms of regional generation characteristics and regional electricity transfers; and, conservative assumptions are used in deriving the BAU scenario compiled for this PDD. It is particular applicable to this study since it can 
isolate the emission intensity for the specific electricity grid for which this project will be implemented into from present to the year 2020.

SECTION E. Estimation of GHG emissions by sources

E.1. Estimate of GHG emissions by sources:

The estimated GHG emissions by source equals zero.

E.2. Estimated leakage:

There is no leakage with this project.

E.3. The sum of E.1 and E.2 representing the project activity emissions:

The project activity emissions equal zero.

E.4. Estimated anthropogenic emissions by sources of greenhouse gases of the baseline: 
E.5. Difference between E.4 and E. 3 representing the emission reductions of the project activity:

E.6. Table providing values obtained when applying formulae above:

SECTION F. Environmental impacts

F.1. Documentation on the analysis of the environmental impacts, including transboundary impacts:

The proposed project will have two main environmental impacts: bird kill and aesthetics. These are discussed below.

\section{Bird Kill:}

The particular region of Jiangsu Province where this project is to be developed is sub tropical, with a rich diversity of wild fowl. As with any wind generation plant, these birds are at increased risk due to the turbines associated with the wind generation plant. Nonetheless, although there is a heightened risk of bird kill due to these turbines, these can not be avoided without significant cost. 
Aesthetics:

Clearly wind turbines are highly visible. However, this wind farm will be situated at relatively far distances from major urbanized areas so that these are not highly prominent features on the landscape.

F.2. If environmental impacts are considered significant by the project participants or the host Party, please provide conclusions and all references to support documentation of an environmental impact assessment undertaken in accordance with the procedures as required by the host Party:

The above environmental impacts are not considered to be significant.

SECTION G. Stakeholders' comments

G.1. Brief description how comments by local stakeholders have been invited and compiled:

Comments have been invited by distributing this PDD to relevant stakeholders.

G.2. Summary of the comments received:

No comments have been received at time of writing 
G.3. Report on how due account was taken of any comments received:

Not applicable. 
Annex I

\section{CONTACT INFORMATION ON PARTICIPANTS IN THE PROJECT_ACTIVITY.}

\begin{tabular}{|l|l|}
\hline Organization: & Carleton University \\
\hline Street/P.O.Box: & Room 449 \\
\hline Building: & Loeb \\
\hline City: & Ottawa \\
\hline State/Region: & Ontario \\
\hline Postfix/ZIP: & \\
\hline Country: & Canada \\
\hline Telephone: & 6135202600 ex 2566 \\
\hline FAX: & \\
\hline E-Mail: & psteenho@ connect.carleton.ca \\
\hline URL: & \\
\hline Represented by: & NA \\
\hline Title: & \\
\hline Salutation: & \\
\hline Last Name: & \\
\hline Middle Name: & \\
\hline First Name: & \\
\hline Department: & \\
\hline Mobile: & \\
\hline Direct FAX: & \\
\hline Direct tel: & \\
\hline Personal E-Mail: & \\
\hline
\end{tabular}




\section{APPENDIX C \\ SUB-SECTOR LEVEL FACTOR CONTRIBUTIONS TO CHANGES IN INDUSTRIAL SECTOR ELECTRICITY DEMAND}

The sub-sector level details are included for the contributions of structure and intensity changes (see figure $\mathrm{C}-1$ ) and fuel share changes (see figure $\mathrm{C}-2$ ) to industrial sector electricity demand in China.

Figure C-1. Contributions of structural and intensity changes to industrial sector electricity demand in China (1998 to 2002)

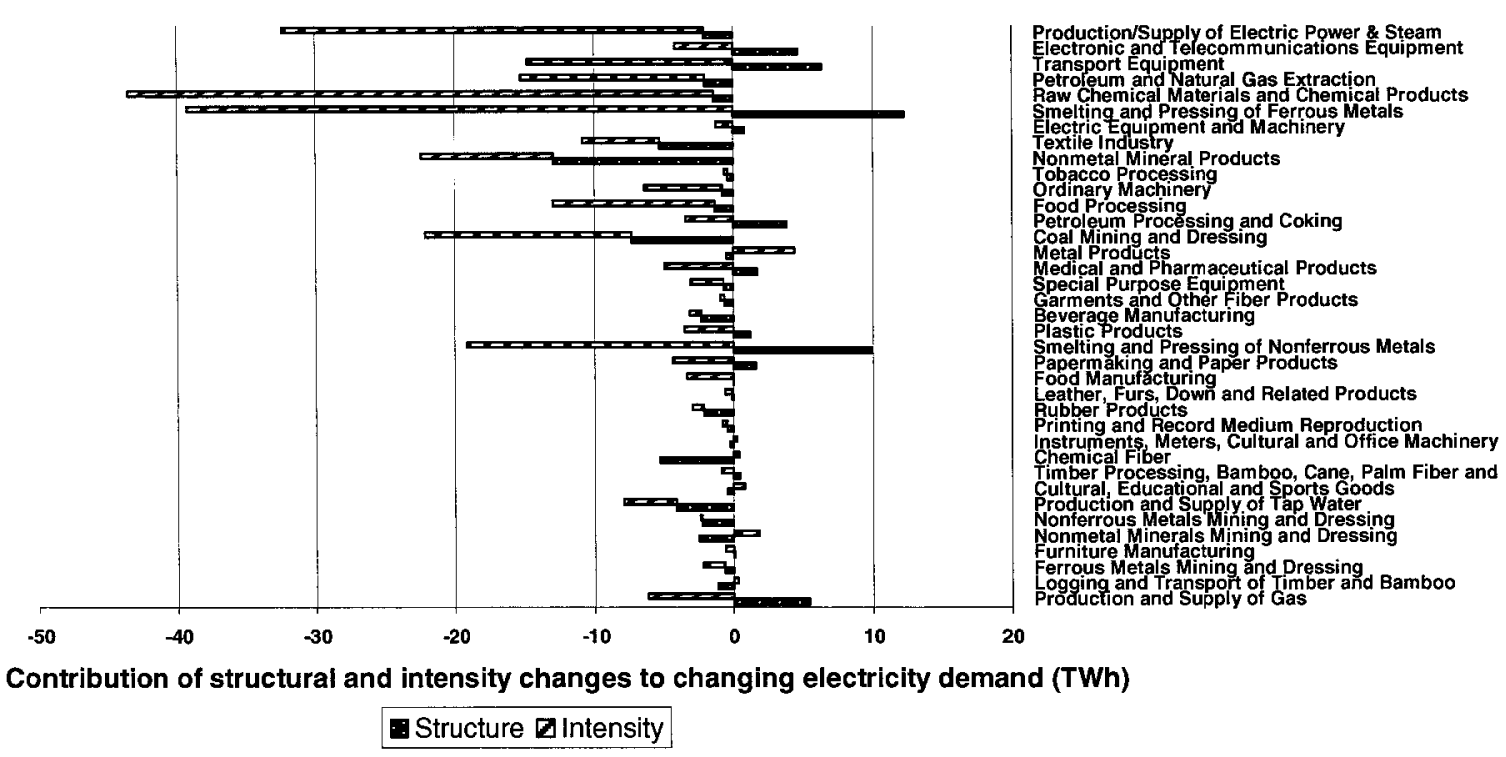


Figure C-2. Contributions of fuel share changes to industrial sector electricity demand in China (1998 to 2002)

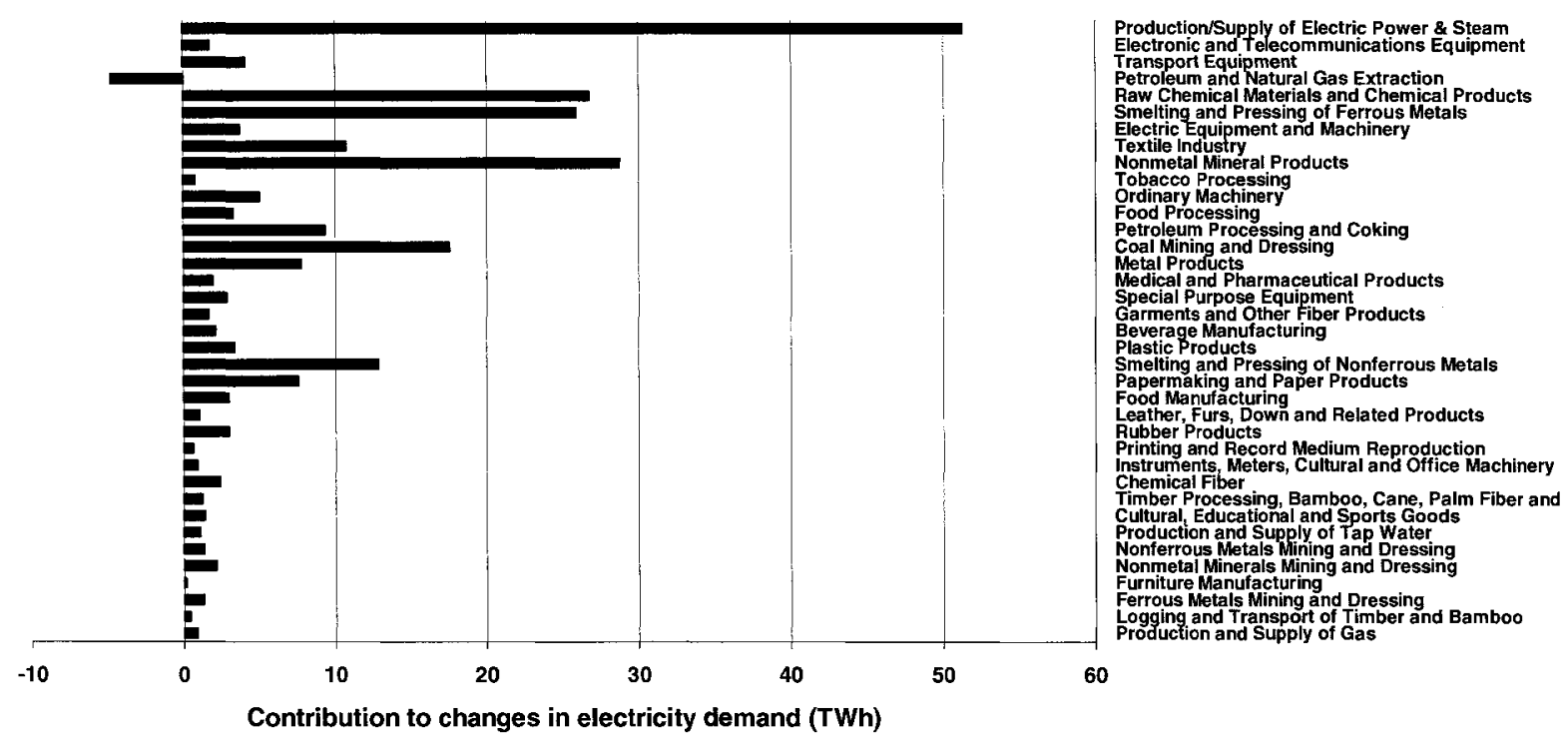




\title{
APPENDIX D \\ Glossary
}

\begin{abstract}
Absolute emissions
Absolute emissions are typically given in $\mathrm{tCO} 2$ equivalent. Baselines expressed in terms of absolute tonnes of $\mathrm{CO}_{2}$ equivalent need assumptions about the activity level in the absence of the project.
\end{abstract}

\section{Additionality}

A CDM project activity is additional if anthropogenic emissions of greenhouse gases by sources are reduced below those that would have occurred in the absence of the registered CDM project activity.

\section{'Annex I' countries}

Annex I countries are the industrialized countries and economies in transition listed in Annex I of the United Nations Framework Convention on Climate Change (UNFCCC or the Convention). Their responsibilities under the Convention are various, and include a non-binding commitment to reducing their greenhouse gas emissions to 1990 levels by the year 2000 .

\section{Baseline}

To determine the number of credits that could be generated by an individual CDM project, an indication is needed of what GHG emissions would have been in the absence of that project (i.e. what would have happened otherwise). The amount of GHG emitted in the hypothetical non-project scenario is referred to as a project's baseline. A baseline is thus a quantification of this hypothetical emission level and may be used for comparative 
purposes to test for the GHG "additionality" of an individual project6. CDM projects will qualify for certified emission reduction units (CERs) if they are additional relative to the baseline.

\section{BAT}

Best available technology. This refers to the implementation of the best available technology available for the particular energy service required.

\section{Baseline dynamics}

Dynamics refers to how changes in one variable or sets of variables may influence other variables which play a role in determining the electricity intensity of electricity generated. Baseline dynamics is a forecast baseline which adjusts to the changes in the business environment over time.

\section{Base-load}

The minimum amount of electric power delivered or required in an electricity grid over a given period of time at a steady rate. 


\section{Base-load Power Plants}

These power plants typically have the lowest operating costs of all plans in a system. This in conjunction to relatively inflexible and costly start ups means these plants are run nearly continuously in an electricity grid.

\section{Build margins (BM)}

Build margin approaches seek to develop a proxy for what type of power plant would have been built (or built sooner) to meet the electricity demand should the CDM electricity project not be implemented. For example, a build margin baseline could be based on plants recently built or currently under construction. The development of such electricity multi-project baselines requires plant specific data on those recent plants/units included in the sample used to calculate the multi-project baseline:

- Source of electricity generation (e.g. natural gas, water, bituminous coal, etc);

- Generating capacity (measured in MW - it is a necessary input to calculate the electricity production in MWh);

- Load factor (for what portion of total possible hours in a year is the plant/unit in operation - this is necessary to determine the electricity production in $\mathrm{MWh}$ );

- Conversion efficiency (for fossil fuels);

- Emission factors (to convert into GHG emissions).

- Commissioning date (in order to determine whether the plant/unit should be used in the sample of recent capacity additions).

- Type of technology (e.g. internal combustion engine, combined cycle gas turbine, etc). 


\section{Business as Usual Scenario (BAU)}

Estimate of a company's future and current emissions under normal operating circumstances. Depending on the scope of the business as usual scenario this may incorporate some emission reduction regulatory controls including carbon taxes etc.

\section{CDM}

Clean Development Mechanism (defined in Article 12 of the Kyoto Protocol). The CDM is a mechanism established by Article 12 of the Kyoto Protocol for project-based emission reduction activities in developing countries. The CDM is designed to meet two main objectives: to address the sustainable development needs of the host country, and to increase the opportunities available to Parties to meet their reduction commitments.

\section{CDM Executive Board}

The CDM's Executive Board is a multilateral regulatory entity by means of which the CDM will be supervised. The Executive Board of the CDM is the only authority with the capacity to issue, control and record Certified Emission Reductions (CERs). The Executive Board will have a complete record of the CERs that may have been transacted through the CDM.

\section{Coal Mine Methane (CMM)}

Methane (global warming potential 21 times that of carbon dioxide) that is released from both coal and coal seams from active or inactive coal mines. 


\section{Dispatch Simulation Model}

A model that simulates the complex operations of the electric system in response to a decrease in demand or an additional supply from a new project. One kWh of electricity savings may affect the operation (and efficiency) of several plants, some on the margin, some not.

\section{Emission Intensity}

The volume of emissions generated per unit of energy combusted/generated.

\section{Ex Ante}

Ex ante evaluation refers to forward-looking assessment of the likely future effects of new policies of proposals. Ex ante assessment differs from ex poste assessment, or backward-looking assessment of the effects of introduced policies of proposals.

\section{Greenfield}

New projects (as opposed to old plants that are refurbished).

\section{Kyoto Units}

Kyoto units refer to the various carbon units that can be used to reduce or offset national emission inventories, including emission reduction units (ERU), certified emission reductions (CER), assigned amount units (AAU), and removal units (RMU). 


\section{Levelized Unit Costs}

Levelized unit costs involve the calculation of the net cost of generating electricity after considering all forms of revenue and expenditures. It is a metric, communicated on a per $\mathrm{kWh}$ basis, which is often used to compare different types of electricity projects to determine which one might be the best investment opportunity.

\section{Load Structure/factor}

Portion of total possible hours in a year is the plant/unit in operation - this is necessary to determine the electricity production in MWh

\section{'Non-Annex I' countries}

Countries not included in Annex I of the United Nations Framework Convention on Climate Change UNFCCC. Non-Annex I countries do not currently have binding emission reduction targets.

\section{Multiple Project Baselines (MPB)}

These baselines can be applied to a number of similar projects, e.g. to all electricity generation CDM or JI projects in the same country. The definition of a multi project 
baseline can be given by formula (UNEP/OECD/IEA, 2001), as shown:

$$
\sum_{i=1}^{n}\left[\frac{G H G \text { emissionsz }}{\sum_{\Sigma=1}^{n} \text { electricity production }}\right]
$$

Where:

- $\mathrm{Z}$ represent each individual power plant/unit in the database:

- GHG emissions for each plant/unit "z" are calculated in tCO2-equivalent

- Electricity production ${ }_{z}$ for each plant/unit "z" in GWh

\section{Operating Margin (OM)}

Operating margin approaches, as defined in Lazarus, Kartha and Bernow (2001) for example, seek to determine which plants are on the operating margin, i.e. the last units to be operated to meet demand, at each hour. This approach is based on the assumption that a CDM/JI electricity project will displace existing power generation on this operating margin. Operating margin information could be obtained using a weighted average marginal emission rate (WAMER) approach or dispatch models. This methodology is quite data intensive. 


\section{Project Design Document (PDD)}

The objective of the project design document is to present information on the projects location, characteristics and methods (including baseline methodology), and it includes a description of why the project is additional.

\section{Project Specific Baselines}

Project-specific emission baselines are those that have been drawn up by examining projects on a case-by-case basis.

\section{Relative Emission Intensity}

Relative baselines measure emission reductions in terms of the emission intensity of a particular project, often given in energy units such as per MWh.

\section{Scenario}

A plausible description of how the future may develop, based on a coherent and internally consistent set of assumptions about key relationships and driving forces (e.g. rate of technology changes, prices). Note that scenarios are neither predictions nor forecast.

\section{Technology Transfer}

The process by which energy-efficient or low emission intensive technologies developed by industrialized nations are made available to less industrialized nations. Technology 
transfer may occur through the sale of technology by private entities, through government programs, non-profit arrangements, or other means.

\section{Transaction fees}

In the context of the Kyoto Mechanisms, transaction costs are e.g. caused by the administrative process and thus depend on the institutional framework. In principal transaction costs of CDM projects can be differentiated into transaction costs arising from undertaking projects in developing countries and transaction costs arising from undertaking a project under the CDM framework. Furthermore transaction costs accrue at different stages in the process of a transaction or project cycle. Undertaking a project under CDM framework will only be viable if the costs of transacting the CERs are substantially lower than the revenue they will generate.

\section{Transmission and distribution losses}

Transmission and distribution losses refer to the amount of electricity lost during its transmission from the generator to sub station, and from the sub station across distribution lines to transformers and the end user. Typically, this value is given in energy balance tables in total energy lost (i.e. MWh), which is then used to show the losses on a percentage basis of total generation.

\section{United Nations Framework Convention on Climate Change (UNFCCC)}

The UNFCCC was established in June 1992 at the Rio Earth Summit. Its primary objective is the "stabilization of greenhouse gas concentrations in the atmosphere at a level that would prevent dangerous anthropogenic (man-made) interference with the 
climate system. Such a level should be achieved within a time-frame sufficient to allow ecosystems to adapt naturally to climate change, to ensure that food production is not threatened, and to enable economic development to proceed in a sustainable manner." The UNFCCC is the governing body for international negotiations. 